\title{
Instructive Function of Surface Structure of Calcium Phosphate Ceramics in Bone Regeneration
}

\author{
Jingwei ZHANG
}
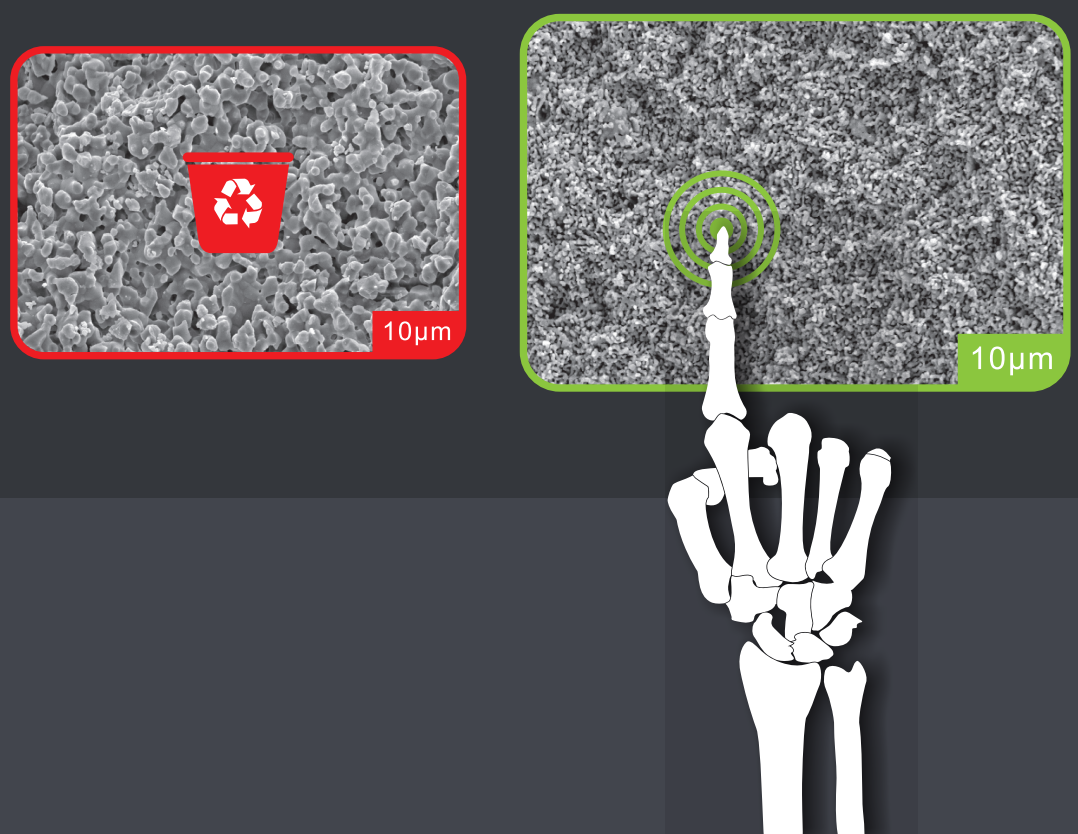


\section{INSTRUCTIVE FUNCTION OF SURFACE STRUCTURE}

\section{OF CALCIUM PHOSPHATE CERAMICS IN BONE REGENERATION}

Jingwei Zhang 
Members of the committee:

Chairman: Prof. Dr. Ir. H. Hilgenkamp

(University of Twente)

Promoter: Prof. Dr. C. A. van Blitterswijk

(University of Twente)

Co-promoter: Dr. H. Yuan

(Maastricht University)

Members:

Prof. Dr. J. Weng

(Southwest Jiaotong University, Chengdu, China)

Prof. Dr. P. Habibovic

(Maastricht University)

Dr. L. Moroni

(Maastricht University)

Dr. J. J. J. P. van den Beucken

Prof. Dr. J.D. de Bruijn

(Radboud University, Nijmegen)

(University of Twente)

\title{
INSTRUCTIVE FUNCTION OF SURFACE STRUCTURE OF CALCIUM PHOSPHATE CERAMICS IN BONE REGENERATION
}

\author{
Jingwei Zhang \\ PhD thesis, University of Twente, Enschede, The Netherlands
}

ISBN: 978-90-365-4062-9

Copyright (c) Jingwei Zhang, Enschede, The Netherlands, 2016. Neither this book nor its parts may be reproduced without permission of the author.

Cover Design: Roni Song (宋雨龙) 


\title{
INSTRUCTIVE FUNCTION OF SURFACE STRUCTURE
}

\section{OF CALCIUM PHOSPHATE CERAMICS IN BONE REGENERATION}

\author{
DISSERTATION \\ to obtain \\ the degree of doctor at the University of Twente, \\ on the authority of the rector magnificus, \\ Prof. Dr. H. Brinksma, \\ on account of the decision of the graduation committee, \\ to be publicly defended \\ on Thursday, February $18^{\text {th }}, 2016$, at $14: 45$ \\ by
}

Jingwei Zhang

Born on April $1^{\text {st }}, 1983$

In Wuchang, Heilongjiang, China 
This dissertation has been approved by:

Supervisor: Prof. Dr. C. A. van Blitterswijk

Co-supervisor: Dr. H. Yuan 


\section{Table of Content}

Chapter 1

General introduction

Chapter 2

Dimension of surface microstructure as an osteogenic factor

in calcium phosphate ceramics

\section{Chapter 3}

Surface structure of calcium phosphate ceramics instructs

inductive bone formation via influencing on morphology

and primary cilia structure of stem cells

\section{Chapter 4}

Calcium phosphate ceramics initiate osteogenic response

through topographical cues

\section{Chapter 5}

Cells responding to surface structure of calcium phosphate ceramics

in bone regeneration

\section{Chapter 6}

Microporous calcium phosphate ceramics driving osteoinduction through

surface architecture

\section{Chapter 7}

General discussion, conclusion and future perspectives

Summary

Samenvatting

List of publications and selected abstracts

Acknowledgement 



\section{Chapter 1}

General introduction 
Chapter 1 


\subsection{Bone}

Bone is the main component of the skeletal system, which is involved in the protection, support and motion of the body. By weight, it is composed of $60 \%$ inorganic components, $30 \%$ organic components and $10 \%$ water. The inorganic part consists of the complexes of calcium phosphates (CaP) in both amorphous (30\%) and crystalline fractions (70\%) [1], it provides compressive strength and plays an important role in calcium homeostasis [2, 3]. The organic part is composed of $90 \%$ type I collagen, which is responsible for the bone tensile properties [4], and 10\% non-collagenous proteins (e.g. proteoglycans, osteopontin, osteonectin, osteocalcin, bone sialoprotein, decorin and biglycan), which are important for bone metabolism.

Bone is a hard but living tissue continuously maintained and renewed. Osteoblasts, osteocytes and osteoclasts are the main bone cells. Osteoblasts synthesize the organic matrix of bone by secreting a wide variety of extracellular matrix (ECM) proteins and produce new bone. They also participate in the mineralization process and in the control of osteoclast function. When an osteoblast is in its terminal differentiation stage, it remains entrapped in its self-produced bone matrix and is called osteocyte. Osteocytes are the most abundant cells in bones and are believed to maintain bone by sensing mechanical strains and bone damage. They have a typical morphology with long thin cytoplasmic processes, which form a fine network of connections with other osteocytes and with the osteoblasts located at the surface of the bone (i.e. the lining cells). Lining cells cover the bone surface and thereby separate the bone surface from the bone marrow. Osteoclasts are located at the bone surface and resorb bone tissue by removing its mineralized matrix and breaking up the organic bone. They are multinucleated giant cells and resorb bone via local acidification and secretion of various proteases [5].

\subsection{Wound healing of bone}

Bone is a highly vascularized tissue with a unique capacity to heal and remodel without leaving a scar after bone damage [6]. Bone healing process involves cascades of biological events, which generate intra and extracellular molecular signals for bone morphogenesis. Healing is divided essentially into four overlapping stages, each one with specific molecular events. Generally, the entities that control bone repair are inflammatory and vascular cells, 


\section{Chapter 1}

osteochondral progenitors, osteoblasts, and osteoclasts. The procedure is driven by special cytokines, angiogenic growth factors, and osteogenic factors. Subsequent to the healing of soft tissues, bone healing involves soft callus formation followed by its maturation into a hard callus, which leads to the final remodeling and establishment of original shape and function of the damaged bone. The mechanism behind the healing process always requires the regulation of chemotaxis, proliferation, differentiation, ECM synthesis, formation and remodeling of the newly formed bone at the injury site.

\subsubsection{First stage of bone healing: inflammation}

The first stage of bone healing process is inflammation. The bone damage causes the loss of integrity of soft tissues, through the interruption of vascularization and distortion of marrow structure. The hemorrhage at the fracture position is first contained and then develops a hematoma. The inflammatory reaction is modulated by several immune system cells, such as platelets, macrophages, granulocytes, lymphocytes and monocytes. The cells associated with inflammatory processes infiltrate the hematoma and combat the infection by secreting cytokines and growth factors, resulting in clot formation.

The inflammatory cytokines that initiate the regeneration cascade are interleukin-1 (IL-1), interleukin-6 (IL-6) and tumor necrosis factor-alpha (TNF-alpha). They are secreted by macrophages, other inflammatory cells and mesenchymal cells [7], and possess a chemotactic effect by enhancing ECM synthesis, stimulating angiogenesis and engaging fibrogenic cells to the wound site. These cytokines intervene not only during the inflammation process but also in the bone dynamics. For instance, TNF-alpha promotes the recruitment of mesenchymal stromal cells (MSCs) and stimulates osteoclastic function [8].

Growth factors that are involved in the inflammatory process are transforming growth factor-beta (TGF-beta), platelet-derived growth factor (PDGF), vascular endothelial growth factor (VEGF) and bone morphogenetic proteins (BMPs). Most of these growth factors interact with specific membrane receptors (serine/threonine) that trigger intracellular signaling pathways, which finally affect gene expression, enabling the up- and downregulation of proteins. TGF-beta is able to control cellular phenomena associated with osteoblast-like cells, and its primary function is to stimulate cell proliferation and bone matrix synthesis [9]. TGF-beta may also play a role in cell differentiation due to its ability to 
control the expression of differentiation commitment genes [10]. PDGF is a polypeptide synthesized essentially by platelets or monocytes, macrophages and osteoblasts [11]. It is a potent mitogenic factor for cells of mesenchymal origin such as osteoblasts [12]. Its release occurs during the early phase of inflammation. PDGF is a chemotactic stimulator for inflammatory cells favoring the migration of MSCs and osteoblasts [13].

Good blood supply to the injury site is essential for bone healing. VEGF is a growth factor specialized in mediating neo-angiogenesis and endothelial-cell specific mitogens [14]. VEGF production is the major coupling mechanism between angiogenesis and osteogenesis during fracture healing due to the key role that blood vessel invasion play. Bone healing could be enhanced by exogenous administration of VEGF. Neutralization of VEGF receptor results in a delay in the vascular occupation and replacement of cartilaginous callus with bone. The importance of VEGF for angiogenesis and bone regeneration at the injury site was proven in several studies $[15,16]$. VEGF not only enhanced angiogenesis but also promoted osteogenic differentiation of osteoblasts [17].

\subsubsection{Second stage: soft callus formation}

This second stage of bone healing is dominated by the activity of three types of cells: MSCs, fibroblasts, and chondrocytes (cartilage forming cells). Mesenchymal progenitors play an important role due to their capacity to differentiate into chondrocytes, being these cells' fate essentially defined by cues from their microenvironment such as cytokines or other biological factors. Fibroblasts aid soft callus formation by producing fibrous tissue to fill the regions where cartilage production is not efficient. Eventually, cartilaginous regions grow and progressively merge to each other to produce a central fibrocartilaginous plug between the fractured fragments of the fracture. The cartilaginous template with bony callus can provide mechanical support to the damage site and the resulting soft callus is essential for further processes of ossification.

Growth factors (e.g. VEGF and TGF) associated with fibroblast proliferation and chondrogenic differentiation were already noticeable during inflammation stage. VEGF promotes invasion of the callus and capillary ingrowth [18] and its expression is regulated by the cartilage regulatory factor Cbfa1/Runx2 [19]. TGF is responsible for cellular responses associated with proliferation of undifferentiated MSCs to chondrocytes. In addition to VEGF, 


\section{Chapter 1}

fibroblast growth factor-1 (FGF-1) and insulin-like growth factor (IGF) are observed in this stage[20].

BMPs are important in the callus formation. For example, BMP-4 is involved in the formation of callus [21] and it has been suggested that the balance between BMP-4 and its antagonist (noggin) could be an important factor in the regulation of callus formation [22]. During the concluding phase of soft callus formation, chondrocytes mature into hypertrophic chondrocytes and undergo a series of biochemical reactions in order to mineralize the cartilage matrix.

\subsubsection{Third stage: hard callus formation}

Hard callus formation is also known as a "primary bone formation". The main phenomena observed during hard callus formation are the high levels of osteoblast activity and the formation of mineralized bone matrix [23]. At this stage the soft cartilaginous callus is gradually removed and replaced by mineralized bone. The hard callus formation is also accompanied by the action of growth factors, where VEGF shows itself to be a growth factor of great importance at this step [15]. De novo bone formation is mediated, among others, by the BMP family.

\subsubsection{Fourth stage: bone remodelling}

The bone formed in the earlier stages of bone healing process will be then remodeled. Osteoclasts and osteoblasts take their responsibility in this stage. Bone is first resorbed by osteoclasts, creating a shallow resorption pit known as a "Howship's lacuna". Then osteoblasts deposit compact bone within the resorption pit. Bone remodeling responds to functional demands derived by mechanical loading as well according to Wolff's law. Eventually, the fracture callus is remodeled into a new shape which closely duplicates the bone's original shape and strength.

\subsection{Bone grafts}

Self-healing of bone can only be achieved in small bone defects (i.e. non-critical-sized bone defects). Bone's own repair mechanism fails in critical-sized defects leading to musculoskeletal disorders. Musculoskeletal disorders of arthritis, osteoporosis, 
osteonecrosis, bone fracture, bone tumor, trauma (due to sporting and road traffic injuries), back pain and other spinal disorders are the major worldwide health problems. They have a substantial impact on the quality of life of the population and it costs over $\$ 126$ billion annually in the U.S. to treat such disorders [24]. For instance, about 6.8 million people come to medical attention for bone fractures each year in the U.S. To repair bone, natural (i.e. autografts, allografts and xenografts) and synthetic bone grafts are often considered as the choices (Figure 1).

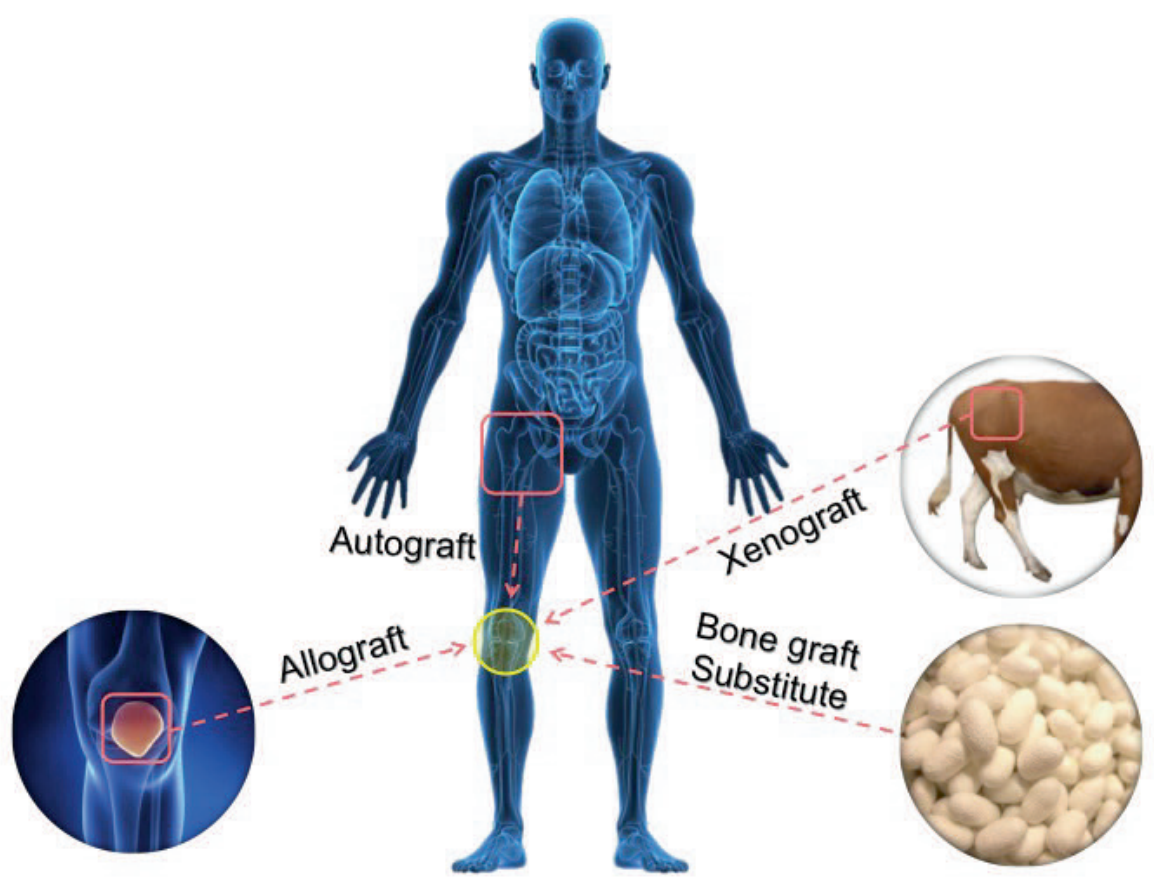

Figure 1. Different types of bone grafts. (A) Autograft: The surgeon harvests bone from another site of the patient's skeleton. $(B, C)$ Allograft and xenograft: The bone graft is obtained from a human donor or animals. (D) Synthetic bone graft substitute: There are different origins for synthetic grafts.

\subsubsection{Natural bone grafts}

Autografts or autologous bone grafts are bone segments taken from one anatomic site and transplanted to another site of the same individual. Autografts have been the gold standard of bone replacement for many years because of their non-immunogenicity and their 


\section{Chapter 1}

osteoconductive and osteoinductive properties $[25,26]$. However, autografts cause donorsite pain and morbidity, need additional surgical time and are limited in amount [27].

Allografts refer to bone transferred from one member into another member of the same species. Conversely, xenografts are harvested from one individual and transplanted into another individual of a different species. The most common sources of xenografts are natural coral, porcine and bovine bone [28]. Neither xenografts nor allografts are ethically questionable, while they may induce immunological reactions and be less effective in terms of bone regeneration [27]. Because natural bone grafts hold these disadvantages, it is of great interest to develop synthetics for bone replacement, bone repair and bone regeneration.

\subsubsection{Synthetic bone graft substitutes}

Synthetics are becoming more and more important in bone regeneration. They are used alone [29], as scaffolds for tissue engineering, or as carriers for growth factors [30] and gene therapy [31] for bone regeneration. Synthetic bone graft substitutes are available in different forms with chemistry of polymers, metals, ceramics and their combinations. Because their chemical composition is similar to the mineral component of natural bone, calcium phosphate $(\mathrm{CaP})$ ceramics have been suggested as promising synthetic bone graft materials for bone regeneration. Hydroxyapatite (HA), $\beta$-tricalcium phosphate $(\beta-T C P)$ or the mixtures of both, named as biphasic calcium phosphate (BCP), are the majority. These materials have long been investigated for bone regeneration. In 1920, Albee and Morrison first reported the bone repair potential of $\mathrm{CaP}$ ceramics during an experiment performed to repair one-quarter inch radial defects in rabbits using injectable TCP [32]. Subsequent investigators have studied CaP ceramics ranging from periodontal to orthopaedic applications in 1970 s [33, 34]. It is generally accepted that CaP ceramics are biocompatible and bioactive, support bone formation on their surface and form a chemical bonding to the newly formed bone [35]. Furthermore, the resorption rate of $\mathrm{CaP}$ ceramics could be adjusted by the content ratio of HA to TCP, where HA is non-resorbable and TCP is resorbable $[29,36]$.

\subsubsection{Osteoinductive CaP ceramics}

To repair critical sized bone defects, both conductive and inductive bone formation are necessary. Autografts are both osteoconductive and osteoinductive, while synthetics are 
generally thought to be osteoconductive. Scientists aim to make bone grafting materials have equivalent osteoinductive capacity as autologous bone does. In addition to mimic autologous bone by introducing osteogenic cells and/or growth factors into synthetics, in the past three decades several specific CaP ceramics were reported to possess the capacity to induce bone formation at ectopic sites without the addition of growth factors and cells [29, $36,37]$. It has been shown that such an osteoinductive property is up to the physicochemical properties of CaP ceramics [38-40].

\subsection{Material factors relevant to osteoinductive CaP ceramics}

\subsubsection{Macrostructures}

First of all, CaP ceramics should have a 3D macroporous structure for osteoinduction to occur in CaP ceramics. Bone formation induced by CaP ceramics was seldom observed on flat ceramic surfaces. The osteoinductive ceramics had either interconnected macropores or well-defined macro-concavities. The macrostructural properties, i.e. the macroporosity, macropore size, macropore shape, and implant geometry, are thought to promote the transport of nutrients and oxygen through blood vessels, which can also bring along cells with the capacity to differentiate into osteoblasts. It has been reported that scaffolds should have highly interconnected macropores with a diameter of $100 \mu \mathrm{m}$ or greater to ensure cell colonization, nutrients and metabolic waste transport [41, 42]. Apart from macropore size, a recent study by Wang and coworkers suggested that the macropore shape and porosity of HA scaffolds play a critical role in vascularization and osteoinduction [43]. In this study, two types of HA scaffolds with complementary macrostructures were fabricated by spheruliteaccumulating and porogen-preparing methods. The histological results showed that new bone tissue was found in the spherulite-accumulating scaffolds 3 and 6 months after implantations, which was better than that observed in the porogen HA-negative scaffolds [43]. Geometry of the implant has also been shown to be important in osteoinduction. Ripamonti et al. showed that bone formation always started in the concave shaped pores and never in the convex shaped pores of HA rods and discs [44, 45]. 


\section{Chapter 1}

\subsubsection{Microstructures}

The presence of microstructure in CaP ceramics surface is of great importance for osteoinduction. The significance of micropores in CaP ceramics was highlighted in the important reports of Yamasaki et al. and Yuan et al., where HA ceramics with micropores were shown to be osteoinductive after subcutaneous [37] and intramuscular [38] implantations in dogs, while no bone formed in those HA ceramics lacking micropores. It has been also reported that the osteoinductive potential of $\mathrm{CaP}$ ceramics increased with increasing microporosity $[29,36]$. For instance, BCP ceramics having higher microporosity (17\%) induced bone formation in goats after intramuscular implantation, while no bone formation was observed in those having lower microporosity (4\%). Apart from micropores and microporosity, the micro-/nano-scale dimension of the surface structures of the CaP ceramics has been shown to have essential effects on the osteoinductive potential of CaP ceramics $[29,39]$.

\subsubsection{Chemistry}

The influence of the chemistry of CaP ceramics on osteoinduction was seen among HA, TCP and BCP with various HA/TCP ratios. When HA and BCP were compared, BCP had a higher osteoinductive potential than HA [46]. When HA, BCP and TCP were compared, the osteoinductive potential of CaP ceramics increased with the TCP content [29]. In some studies, inductive bone formation occurred only in CaP ceramics having certain HA/TCP ratios [47].

\subsection{A suggestive mechanism of material-driven osteoinduction}

The mechanism of CaP ceramic-driven osteoinduction is not fully understood, while a few hypotheses have been suggested. Most often-referred explanations correlated osteoinduction to protein adsorption, followed by the ion release and surface reprecipitation. Since some BMPs are osteoinductive and CaP ceramics have high affinity to such proteins $[29,36,38,48]$, it is generally thought that CaP ceramics firstly concentrate growth factor (including BMPs from body fluids after implantation) and the induction of bone formation is a secondary response to protein adsorption [49-51]. The protein adsorption theory could explain the phenomenon that only CaP ceramics having micropores gave rise to inductive bone formation and that the osteoinductive potential increased with 
microporosity, because both the presence of micropores and the increase of microporosity enlarged the surface area favoring the concentration of higher amounts of growth factors.

In addition, the $\mathrm{Ca}$ and $\mathrm{P}$ ions released from $\mathrm{CaP}$ ceramics could subsequently re-precipitate to form a biological apatite layer on their surface to support bone formation [39, 52-54]. During the re-precipitation, proteins (including growth factors) could be entrapped into the biological apatite layer. Meanwhile, it has been found that, with the increase of $\mathrm{Ca}$ concentration, stem cells could undergo osteogenic differentiation. It is, thus, suggested that ion release of $\mathrm{Ca}$ and $\mathrm{P}$, and the subsequent formation of biological apatite layer, play a role in osteoinduction of CaP ceramics. The ion release and re-precipitation theory explains how the chemistry of CaP ceramics affects its osteoinduction. With the increase of the TCP phase in CaP ceramics, more ions were released. The higher concentration of $\mathrm{Ca}$ and $\mathrm{P}$ generated from high TCP content may, on the one hand, induce/enhance osteogenic differentiation of stem cells and, on the other, enhance the re-precipitation to concentrate locally higher amounts of proteins (including growth factors) in the biological apatite layer.

The ion release and re-precipitation theory could also explain the role of microspores and microporosity on osteoinduction of CaP ceramics. The increase of surface area by the presence of micropores and the corresponding increase in microporosity, facilitate ion release and re-precipitation which favor osteogenesis.

The protein adsorption and ion release/precipitation theory could also explain the role of macrostructure in CaP ceramic driven osteoinduction. The macrostructure is believed to allow infiltration of nutrients, oxygen and cells; meanwhile it enlarges the surface available for adsorption and ion exchange. The protein adsorption and ion release/precipitation theory emphasizes the role of soluble chemical cues (growth factors and ions) on the osteogenic differentiation of stem cells. Increasing evidences are now showing the crucial role of surface structure in osteogenic differentiation of stem cells, as well as material-driven osteoinduction. 


\subsection{Biological functions of material surface}

\subsubsection{MSCs as a tool}

MSCs have the ability to differentiate into various cell types including osteoblasts $[55,56]$, adipocytes [57, 58], chondrocytes [59], smooth muscle cells [60] and neurons [61, 62]. Differentiation of MSCs normally requires the presence of differentiation factors by the use of supplemented mediums that might contain growth factors or cytokines (e.g. dexamethasone for osteogenic differentiation, insulin for adipogenic differentiation, and hydrocortisone for smooth muscle cell differentiation) $[63,64]$. The in vivo environment of the cells is characterized by complex chemical and physical cues. This environment could be mimicked in vitro culture systems where cells are cultured on structured surfaces (change of one or two factors) in culture medium with supplements of biochemical factors. Cells may encounter different sizes and shapes of structures, ranging from the macro- to the microand nano-scale, where each could be a key factor affecting cell behavior and functionality. It is becoming increasingly evident that MSCs are highly sensitive to their microenvironment and will respond to the factors, i.e. surface topography $[65,66]$, mechanical factors $[67,68]$ and surface roughness $[69,70]$, other than soluble chemical cues (e.g. growth factors).

\subsubsection{Regulation of MSCs by surface structure}

Micro- and nano-scale surface topography is attractive in various applications, such as tissue engineering, implant design, high-throughput microarrays and fundamental cell biology. It has also been shown that micro- and nano-scale topographies can modulate cellular behaviors.

\subsubsection{Micro-scale surface topography}

Micro-scale topography could direct cell activities because most of the cells are developed at the micro-scale [71-73]. To investigate the effect of micro-scale topographical cues on cell responses, different surface topographies were produced including wells [74], grooves [7577] and pillars [76]. Different cellular responses to micro-scale surface topography were investigated regarding cell shape [77], cell proliferation [78], cell differentiation [75, 77] and protein expression [79]. 
Several key studies have reported the effect of the dimension of topographical cues on the MSCs responses [80-82]. For instance, McBeath et al. showed that MSCs cultured on flat fibronectin coated islands with size ranging from 1,024 to $10,000 \mu^{2}$ switched from adipogenic to osteogenic phenotype when in contact with increasing island size [82]. On the small islands cells were round, while on the bigger islands they were significantly more spread, highlighting the relevance between cell shape and cell differentiation. A follow up study by Gao et al. [80] showed that the size of the islands also conferred a switch between chondrogenic and smooth muscle cell (SMC)'s fate, which was mediated by cell shape, Rac1 and $\mathrm{N}$-cadherin, pointing out the tight coupling between lineage commitment and the changes in cell shape, cell-interface adhesion and cell-cell adhesion. A recent study on the effect of the width of micro-scale channels on MSCs fate revealed that, by increasing the width of micro-channels from $30 \mu \mathrm{m}$ up to $80 \mu \mathrm{m}$, it was possible to alter the morphology and differentiation of MSCs [75]. It was shown that MSCs were significantly more aligned and elongated on the narrower micro-channels $(30 \mu \mathrm{m})$ in contrast to those on wider microchannels $(80 \mu \mathrm{m})$. The myogenic differentiation of MSCs in this study was observed on the substrates with narrow micro-scale channels $(30 \mu \mathrm{m})$, as indicated by the up-regulation of myogenic genes of MyoD1, GATA4, MHC7 and NKx2.5.

Apart from the size of micro-scale topography, the shape of micro-scale topography has an influence on cell response as well. Kilian et al. [81] have shown that specific differentiation profiles of MSCs could be obtained when cultured on mixed shapes with a range of geometric features and having various areas. In particular, an effect of subtle geometric shape on the differentiation of MSCs was shown: features between concave regions can promote increased myosin contractility which enhances the osteogenic differentiation of hBMSCs.

\subsubsection{Nano-scale surface topography}

A growing number of nanofabrication techniques have been developed and used to tailor materials' surface topography at the nanometer dimension. Thus more evidence is being gathered on the importance of nano-scale topography in cell response. Cells are likely able to respond to nanostructures, since ECM contains nanoscale collagen fibrils while cellular receptors and filopodia are at the nanoscale. Increasing evidence is also demonstrating that 


\section{Chapter 1}

nano-scale topographical cues alone could directly regulate cellular behavior in the absence of any inductive biological agents [83-88]. For instance, nanopillar matrices enhanced osteogenic differentiation of MSCs as compared to flat substrates [85].

Several studies have focused on the behavior of MSCs on surface with various nanostructure sizes $[86,88-90]$. Oh et al. studied the effects of $\mathrm{TiO}_{2}$ nanotube dimensions on MSCs fate [89]. The adhesion, elongation and differentiation of MSCs were altered by increasing the diameter of the nanotubes from $30 \mathrm{~nm}$ up to $100 \mathrm{~nm}$. In particular, the elongation ratio of MSCs increased but the number of cells decreased with increasing size of $\mathrm{TiO}_{2}$ nanotubes. The substrates displaying $30 \mathrm{~nm}$ nanotubes had a higher number of adherent cells with a more round morphology, in contrast to MSCs cultured on $100 \mathrm{~nm}$ nanotubes which were more spread. Osteogenic differentiation of MSCs in this study was observed to occur on the $\mathrm{TiO}_{2}$ nanotubes with the diameter of $70 \mathrm{~nm}$ and $100 \mathrm{~nm}$, while negligible amounts of osteogenic markers observed on carbon nanotubes of $30 \mathrm{~nm}$ and $50 \mathrm{~nm}$. Further studies performed by Khang et al. have shown that the MSCs respond differently to sub-nano, nano and submicron hybrid titanium surfaces [90]. After 4 and 24 hours of culture, a significant greater cell attachment and more cells with better spread and aligned morphology were observed on the nano and submicron surfaces as compared to the sub-nano one. Moreover, MSCs displayed more focal adhesion contacts (vinculin) and better organized cytoskeletons (f-actin) when cultured on the nano and submicron surfaces than those seeded on the subnano surface at both $4 \mathrm{~h}$ and $24 \mathrm{~h}$ of culture. The expression of osteogenic genes was notably increased in MSCs grown on submicron surfaces compared to those on sub-nano and nano titanium surfaces. These results seem to indicate that an increase in the size of features in the range of sub-nano to submicron scales has a positive impact on osteogenic differentiation of MSCs.

\subsubsection{Regulation of MSCs by surface roughness}

In addition to micro-/nano- structured surface topography, surface structure may affect cell behaviors via surface roughness. Cell attachment [70], proliferation [91, 92] and differentiation were found to be related to the surface roughness of materials [93, 94]. For instance, a combination of high surface roughness and low stiffness of the substrate appeared to be the most favorable for cell attachment of MSCs [70]. Rough surfaces with 
low stiffness were superior to smooth surfaces with high stiffness in promoting the osteogenic induction of MSCs, determined by increased ALP activity and Ca deposition. In addition, increased osteogenic differentiation of MSCs, measured by up-regulated gene osteogenic markers (SPP1, RUNX2 and BSP) and deposits of calcified matrix, is associated with decreased proliferation on rough Titanium (Ti) surface versus smooth Ti surfaces in vitro [95]. Furthermore, calcified matrix deposition was detected at earlier time points on rough $\mathrm{Ti}$ surfaces compared to smooth $\mathrm{Ti}$ surfaces, which can be correlated with the increased expression of osteogenic promoter WNT5A on rough surfaces [95].

\subsubsection{Regulation of MSCs by surface stiffness}

Apart from surface topographical cues, there is significant evidence to show the critical role of surface mechanical factors in controlling MSCs fate and lineage determination [96]. The initiating event in the regeneration of specific tissues is the transition of the undifferentiated cells into differentiated tissue-forming cells, which is a process driven by sequential activation of diverse signaling pathways and transcription factors controlling the expression of specific genes. Studies to date indicated that the cytoskeletal motors are modulated by mechanical factors of surface [97], and that the subsequent changes in both actin structures and the formation of focal adhesion are linked to changes in MSCs differentiation. The cellular mechano-transducers could generate signals based on the stiffness that the cells generate from the matrix. Researchers previously reported that one or all of the non-muscle myosin II isoforms (NMM IIA, B, and C) are likely to be involved in the matrix elasticity sensing that drives cell differentiation $[63,82]$.

Cell proliferation and differentiation, and the tissue formation, are highly regulated by initial cell attachment and morphology. Several studies have shown that the shape of MSCs is determined by mechanical factors $[63,98]$. A key study by Fu et al. showed that patterned microposts with various heights and constant diameter affect the shape of MSCs through mechanical factors (Figure 2) [98]. Changes of mechanical stiffness by varying the height of these posts can be achieved by increasing the height that leads to decreased stiffness. MSCs cultured on shorter microposts $(0.97 \mathrm{~mm})$ showed spread morphology, while MSCs cultured on higher microposts showed round morphology (Fig. 2). 


\section{Chapter 1}
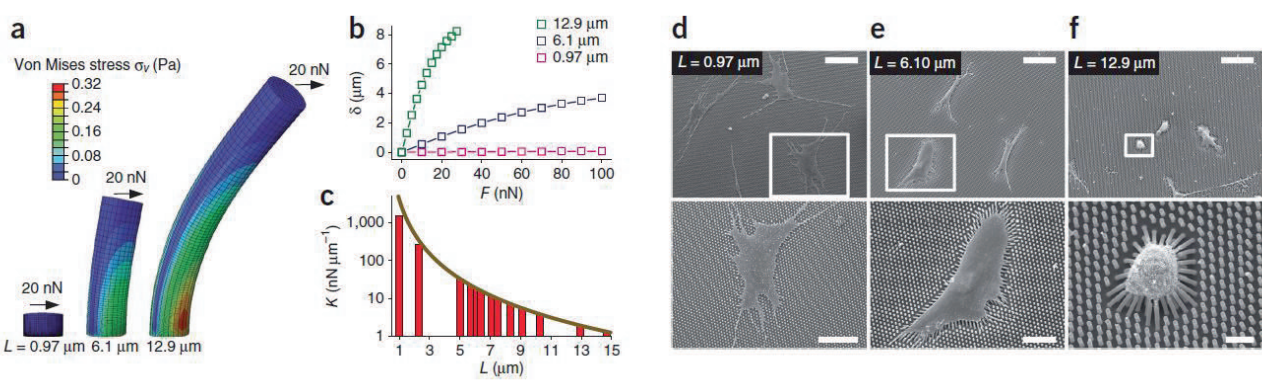

Figure 2. Engineered substrated rigidity by micromolded elastometric micropost arrays. (a) Finite-element method (FEM) analysis of microposts of height (L) the bending in response to applied horizontal traction force (F) of $20 \mathrm{nN}$. (b) Micropost deflection $\delta$ refers to a function of $F$, as calculated by FEM analysis. (c) Nominal spring constant ( $K$ ) as a function of $L$, as computed from FEM analysis (bars) the Euler-Bernoulli beam theory (curve). $K$ measures micropost rigidity. (d-f) scanning electron micrographs of MSCs plated on PDMS micropost arrays of the indicated heights. High magnifications of the top images are shown at the bottom. Scale bars, $100 \mu \mathrm{m}$ (d-f, top), $50 \mu \mathrm{m}$ (d, bottom), $30 \mu \mathrm{m}$ (e, bottom) and $10 \mu \mathrm{m}$ (f, bottom) [98].

Together with the morphological change of cells, studies have shown that MSCs differentiation was determined by surface mechanical factors. For example, Engler et al. have demonstrated that stiffness is critical for the differentiation of naive MSCs [63]. When MSCs were cultured on matrix having various stiffness (0.1-1 $\mathrm{kPa}, 8-17 \mathrm{kPa}$, and $25-40 \mathrm{kPa})$, ranging from brain elasticity (soft) to the crosslinked collagen of osteoids (stiff) [63], MSCs exhibited an increasingly branched, filopodia-rich morphology similar to neuron on soft matrices and had shapes similar to osteoblasts on stiff ones. In the mid-range stiff matrices, MSCs were spindle-shaped and similar to myoblasts. Discher et al. cultured MSCs on matrix having varied stiffness and showed that elasticity is a powerful mechanical cue in directing MSC fate [99]. Stiff matrices led to enhanced cytoskeletal tension and osteogenesis, while softer matrices directed MSCs towards alternative lineages. Interestingly, synthetic and natural matrices that had a comparable effect on cell fate also shared a similar stiffness. Moreover, the effect of matrix stiffness on MSCs was also observed in 3D scaffolds. Recent study by Her et al. demonstrated that the differentiation of MSCs were determined by matrix stiffness of 3D porous scaffolds synthesized by type I collagen and hyaluronic acid [100], which can be controlled in the range of 1-10 kPa from soft to stiff substrates using 1-ethyl-3 (3-dimethylaminopropyl) carbodiimide (EDC) of different concentrations for crosslinking. The 
results showed that matrices with a stiffness of $1 \mathrm{kPa}$ directed MSCs to differentiate into neuronal lineage, while the substrates with a stiffness of $10 \mathrm{kPa}$ induced MSCs into glial cells.

\subsection{Outline of the thesis}

Being chemically similar to the inorganic components of bone, $\mathrm{CaP}$ ceramics have long been attractive as bone graft substitutes. It has been demonstrated that CaP ceramics are in general biocompatible and bioactive and could be made resorbable [29, 36]. Having specific physicochemical properties, CaP ceramics become osteoinductive [46, 101]. Moreover, osteoinductive CaP ceramics have been demonstrated to be able to repair critical-size bone defects $[102,103]$ and could be clinically useful in bone regeneration.

It has long been suggested that ion exchange [104] (i.e. Ca and P ions in the medium), formation of apatite layer on the surface [103] and protein adsorption [105] play important roles in the osteoinduction of CaP ceramics. Recently, the instructive effect of the material surface on cellular behaviors has been widely suggested and confirmed using in vitro model of stem cells. This thesis is thus intended at exploring the instructive roles of surface micro/sub-micron structure of CaP ceramics in bone formation.

To identify the role of surface structure in bone formation (e.g. in inductive bone formation), in Chapter 2, we optimized two TCP ceramics with the same chemistry and similar microporosity but different scale of micropores (TCP-S vs TCP-B). The materials were subjected to both in vitro studies with human bone marrow MSCs (hBMSCs) and in vivo studies with ectopic implantation. Cell proliferation and osteogenic differentiation were targeted in vitro, while the osteoinductive properties of the materials were focused in vivo. To distinguish the role of surface topography and chemical cues in osteogenesis, a trans-well culture model was applied as well. In Chapter 3, using a 2D system of surface structured TCP ceramics and non-surface structured glass slides, we tried to study how hBMSCs respond to surface structure and undergo osteogenic differentiation. In this study the possible correlation between surface topography, cell morphology, primary cilium development, cell differentiation and bone forming ability of CaP ceramics were targeted. Although TCP-B and TCP-S have the same chemistry and similar microporosity, they have various surface areas leading to the chemical changes both inside the materials and the surrounding environments 


\section{Chapter 1}

(e.g. ion release, re-precipitation and protein adsorption). Therefore, the instructive function could come from both the chemical and physical cues of CaP ceramics. To further split the instructive roles of chemical and physical cues, in Chapter 4, we developed another system by gold and carbon coating both structured TCP ceramics and non-surface structured glass slides and performed in vitro studies with hBMSCs.

The final goal of developing instructive materials for bone regeneration is to repair bone defects with such instructive materials. Bone repair follows a general wound healing process with the involvement of cells other than stem cells. In chapter 5, we investigated the response of other six possible bone-related cells to surface structure, aiming the possible responses of inducible cells, osteoprogenitor cells and osteoblasts at different mature stage to surface structure.

To further confirm the role of physical cues of surface structure in bone formation, BCP ceramics (BCP-P and BCP-D) were optimized in chapter 6 with the same chemistry, surface area per volume, profile of protein adsorption, ion release and re-precipitation but various macropore and surface structure for in vivo study using an osteoinduction study model.

By summarizing and discussing the results we got in the studies, the main conclusions and proposed further directions of instructive materials were provided in Chapter 7. 


\section{References}

[1] Blokhuis TJ, Termaat MF, den Boer FC, Patka P, Bakker FC, Henk JTM. Properties of calcium phosphate ceramics in relation to their in vivo behavior. Journal of Trauma-Injury, Infection, and Critical Care. 2000;48:179.

[2] Erlebacher A, Filvaroff EH, Gitelman SE, Derynck R. Toward a molecular understanding of skeletal development. Cell. 1995;80:371-8.

[3] Zaidi M. Skeletal remodeling in health and disease. Nature medicine. 2007;13:791-801.

[4] Little N, Rogers B, Flannery M. Bone formation, remodelling and healing. Surgery (Oxford). 2011;29:141-5.

[5] Bohner M. Resorbable biomaterials as bone graft substitutes. Materials Today. 2010;13:24-30.

[6] Fawcett DW, Bloom W. A textbook of histology: Saunders Philadelphia; 1986.

[7] Kon T, Cho TJ, Aizawa T, Yamazaki M, Nooh N, Graves D, et al. Expression of Osteoprotegerin, Receptor Activator of NF-KB Ligand (Osteoprotegerin Ligand) and Related Proinflammatory Cytokines During Fracture Healing. Journal of Bone and Mineral Research. 2001;16:1004-14.

[8] Barnes GL, Kostenuik PJ, Gerstenfeld LC, Einhorn TA. Growth factor regulation of fracture repair. Journal of Bone and Mineral Research. 1999;14:1805-15.

[9] Joyce ME, Jingushi S, Bolander M. Transforming growth factor-beta in the regulation of fracture repair. The Orthopedic clinics of North America. 1990;21:199-209.

[10] Border WA, Ruoslahti E. Transforming growth factor-beta in disease: the dark side of tissue repair. Journal of Clinical Investigation. 1992;90:1.

[11] Andrew J, Hoyland J, Freemont A, Marsh D. Platelet-derived growth factor expression in normally healing human fractures. Bone. 1995;16:455-60.

[12] Canalis E, McCarthy TL, Centrella M. Effects of platelet-derived growth factor on bone formation in vitro. Journal of cellular physiology. 1989;140:530-7.

[13] Lieberman JR, Daluiski A, Einhorn TA. The role of growth factors in the repair of bone. The Journal of Bone \& Joint Surgery. 2002;84:1032-44.

[14] Ferrara N, Davis-Smyth T. The biology of vascular endothelial growth factor. Endocrine reviews. 1997;18:425.

[15] Street J, Bao M, Bunting S, Peale FV, Ferrara N, Steinmetz H, et al. Vascular endothelial growth factor stimulates bone repair by promoting angiogenesis and bone turnover. Proceedings of the National Academy of Sciences of the United States of America. 2002;99:9656-61.

[16] Peng H, Usas A, Olshanski A, Ho AM, Gearhart B, Cooper GM, et al. VEGF improves, whereas sFlt1 inhibits, BMP2-induced bone formation and bone healing through modulation of angiogenesis. Journal of Bone and Mineral Research. 2005;20:2017-27.

[17] Keramaris NC, Calori GM, Nikolaou VS, Schemitsch EH, Giannoudis PV. Fracture vascularity and bone healing: A systematic review of the role of VEGF. Injury. 2008;39, Supplement 2:S45-S57.

[18] Street J, Bao M, deGuzman L, Bunting S, Peale FV, Jr., Ferrara N, et al. Vascular endothelial growth factor stimulates bone repair by promoting angiogenesis and bone turnover. Proc Natl Acad Sci U S A. 2002;99:965661.

[19] Zelzer E, Glotzer DJ, Hartmann C, Thomas D, Fukai N, Soker S, et al. Tissue specific regulation of VEGF expression during bone development requires Cbfa1/Runx2. Mechanisms of development. 2001;106:97-106.

[20] Abd El Kader T, Kubota S, Anno K, Tanaka S, Nishida T, Furumatsu T, et al. Direct interaction between CCN family protein 2 and fibroblast growth factor 1. Journal of cell communication and signaling. 2014;8:157-63.

[21] Kloen P, Di Paola M, Borens O, Richmond J, Perino G, Helfet DL, et al. BMP signaling components are expressed in human fracture callus. Bone. 2003;33:362-71.

[22] Yoshimura Y, Nomura S, Kawasaki S, Tsutsumimoto T, Shimizu T, Takaoka K. Colocalization of noggin and bone morphogenetic protein-4 during fracture healing. Journal of bone and mineral research : the official journal of the American Society for Bone and Mineral Research. 2001;16:876-84.

[23] Schindeler A, McDonald MM, Bokko P, Little DG. Bone remodeling during fracture repair: The cellular picture. Seminars in cell \& developmental biology: Elsevier; 2008. p. 459-66.

[24] Lowry J. Bone regeneration and repair: Biology and clinical applications. Annals of The Royal College of Surgeons of England. 2006;88:334. 


\section{Chapter 1}

[25] Kanzler B, Kuschert SJ, Liu Y-H, Mallo M. Hoxa-2 restricts the chondrogenic domain and inhibits bone formation during development of the branchial area. Development. 1998;125:2587-97.

[26] Barradas A, Lachmann K, Hlawacek G, Frielink C, Truckenmoller R, Boerman OC, et al. Surface modifications by gas plasma control osteogenic differentiation of MC3T3-E1 cells. Acta biomaterialia. 2012.

[27] Bohner M, Galea L, Doebelin N. Calcium phosphate bone graft substitutes: Failures and hopes. Journal of the European Ceramic Society. 2012;32:2663-71.

[28] DeVore DT. Collagen xenografts for bone replacement: the effects of aldehyde-induced cross-linking on degradation rate. Oral Surgery, Oral Medicine, Oral Pathology. 1977;43:677-86.

[29] Yuan H, Fernandes H, Habibovic P, De Boer J, Barradas A, De Ruiter A, et al. Osteoinductive ceramics as a synthetic alternative to autologous bone grafting. Proceedings of the National Academy of Sciences of the United States of America. 2010;107:13614-9.

[30] Aquino-Martinez R, Rodriguez-Carballo E, Gamez B, Artigas N, Carvalho-Lobato P, Manzanares-Cespedes $\mathrm{MC}$, et al. Mesenchymal stem cells within gelatin/CaSO4 scaffolds treated ex-vivo with low doses of BMP-2 and WNT-3a increase bone regeneration. Tissue engineering Part A. 2015.

[31] Kimelman N, Pelled G, Gazit Z, Gazit D. Applications of gene therapy and adult stem cells in bone bioengineering. Regenerative medicine. 2006;1:549-61.

[32] Albee FH. Studies in bone growth: triple calcium phosphate as a stimulus to osteogenesis. Annals of surgery. 1920;71:32.

[33] Levin MP, Getter L, Cutright DE, Bhaskar S. Biodegradable ceramic in periodontal defects. Oral Surgery, Oral Medicine, Oral Pathology. 1974;38:344-51.

[34] Bhaskar SN, Brady JM, Getter L, Grower MF, Driskell T. Biodegradable ceramic implants in bone: electron and light microscopic analysis. Oral Surgery, Oral Medicine, Oral Pathology. 1971;32:336-46.

[35] Daculsi G. Biphasic calcium phosphate concept applied to artificial bone, implant coating and injectable bone substitute. Biomaterials. 1998;19:1473-8.

[36] Habibovic P, Yuan H, Van Der Valk CM, Meijer G, Van Blitterswijk CA, De Groot K. 3D microenvironment as essential element for osteoinduction by biomaterials. Biomaterials. 2005;26:3565-75

[37] Yamasaki H, Sakai H. Osteogenic response to porous hydroxyapatite ceramics under the skin of dogs. Biomaterials. 1992;13:308-12.

[38] Yuan H, Kurashina K, de Bruijn JD, Li Y, De Groot K, Zhang X. A preliminary study on osteoinduction of two kinds of calcium phosphate ceramics. Biomaterials. 1999;20:1799-806.

[39] Habibovic P, Sees TM, van den Doel MA, van Blitterswijk CA, de Groot K. Osteoinduction by biomaterialsphysicochemical and structural influences. Journal of Biomedical Materials Research Part A. 2006;77:747-62.

[40] Habibovic P, Yuan H, van den Doel M, Sees TM, van Blitterswijk CA, de Groot K. Relevance of Osteoinductive Biomaterials in Critical-Sized Orthotopic Defect. Journal of Orthopaedic Research. 2006;24:86776.

[41] Karageorgiou V, Kaplan D. Porosity of 3D biomaterial scaffolds and osteogenesis. Biomaterials. 2005;26:5474-91.

[42] Lee YJ, Lee J, Park Y, Yoon K. Synthesis of large monolithic zeolite foams with variable macropore architectures. Advanced Materials. 2001;13:1259-63.

[43] Wang H, Zhi W, Lu X, Li X, Duan K, Duan R, et al. Comparative studies on ectopic bone formation in porous hydroxyapatite scaffolds with complementary pore structures. Acta biomaterialia. 2013;9:8413-21.

[44] Ripamonti U, Crooks J, Kirkbride A. Sintered porous hydroxyapatites with intrinsic osteoinductive activity: geometric induction of bone formation. 1999.

[45] Ripamonti U, Crooks J, Tucker M, Sampath T, Rueger D, Reddi A. Long-Term Evaluation of Bone Formation by Osteogenic Protein 1 in the Baboon and Relative Efficacy of Bone-Derived Bone Morphogenetic Proteins Delivered by Irradiated Xenogeneic Collagenous Matrices. Journal of Bone and Mineral Research. 2000;15:1798809.

[46] Yuan H, Van Blitterswijk C, De Groot K, De Bruijn J. A comparison of bone formation in biphasic calcium phosphate (BCP) and hydroxyapatite (HA) implanted in muscle and bone of dogs at different time periods. Journal of Biomedical Materials Research Part A. 2006;78:139-47.

[47] Kurashina K, Kurita H, Wu Q, Ohtsuka A, Kobayashi H. Ectopic osteogenesis with biphasic ceramics of hydroxyapatite and tricalcium phosphate in rabbits. Biomaterials. 2002;23:407-12. 
[48] Urist MR, Huo YK, Brownell AG, Hohl WM, Buyske J, Lietze A, et al. Purification of bovine bone morphogenetic protein by hydroxyapatite chromatography. Proceedings of the National Academy of Sciences of the United States of America. 1984;81:371.

[49] Ripamonti U. Osteoinduction in porous hydroxyapatite implanted in heterotopic sites of different animal models. Biomaterials. 1996;17:31-5.

[50] Yang Z, Yuan H, Tong W, Zou P, Chen W, Zhang X. Osteogenesis in extraskeletally implanted porous calcium phosphate ceramics: variability among different kinds of animals. Biomaterials. 1996;17:2131-7.

[51] Wang J, Chen Y, Zhu X, Yuan T, Tan Y, Fan Y, et al. Effect of phase composition on protein adsorption and osteoinduction of porous calcium phosphate ceramics in mice. Journal of biomedical materials research Part $A$. 2014; 102:4234-43.

[52] Fujibayashi S, Neo M, Kim H-M, Kokubo T, Nakamura T. Osteoinduction of porous bioactive titanium metal. Biomaterials. 2004;25:443-50.

[53] Marques P, Serro A, Saramago B, Fernandes A, Magalhaes M, Correia R. Mineralisation of two phosphate ceramics in HBSS: role of albumin. Biomaterials. 2003;24:451-60.

[54] Garnett J, Dieppe P. The effects of serum and human albumin on calcium hydroxyapatite crystal growth. Biochem J. 1990;266:863-8.

[55] Barradas AM, Monticone V, Hulsman M, Danoux C, Fernandes H, Tahmasebi Z, et al. Molecular mechanisms of biomaterial-driven osteogenic differentiation in human mesenchymal stromal cells. Integrative biology : quantitative biosciences from nano to macro. 2013;5:920-31.

[56] Dalby MJ, Gadegaard N, Tare R, Andar A, Riehle MO, Herzyk P, et al. The control of human mesenchymal cell differentiation using nanoscale symmetry and disorder. Nat Mater. 2007;6:997-1003.

[57] Cuaranta-Monroy I, Simandi Z, Kolostyak Z, Doan-Xuan Q-M, Poliska S, Horvath A, et al. Highly efficient differentiation of embryonic stem cells into adipocytes by ascorbic acid. Stem Cell Research. 2014;13:88-97.

[58] Keck M, Kober J, Riedl O, Kitzinger HB, Wolf S, Stulnig TM, et al. Power assisted liposuction to obtain adipose-derived stem cells: Impact on viability and differentiation to adipocytes in comparison to manual aspiration. Journal of Plastic, Reconstructive \& Aesthetic Surgery. 2014;67:e1-e8.

[59] Meretoja VV, Dahlin RL, Wright S, Kasper FK, Mikos AG. The effect of hypoxia on the chondrogenic differentiation of co-cultured articular chondrocytes and mesenchymal stem cells in scaffolds. Biomaterials. 2013;34:4266-73.

[60] Zheng X, Wu Y, Zhu L, Chen Q, Zhou Y, Yan H, et al. Angiotensin II promotes differentiation of mouse embryonic stem cells to smooth muscle cells through PI3-kinase signaling pathway and NF-KB. Differentiation. 2013;85:41-54

[61] Konagaya S, Iwata $\mathrm{H}$. Microencapsulation of dopamine neurons derived from human induced pluripotent stem cells. Biochimica et Biophysica Acta (BBA) - General Subjects. 2015;1850:22-32.

[62] Bai W-F, Xu W-C, Feng Y, Huang H, Li X-P, Deng C-Y, et al. Fifty-Hertz electromagnetic fields facilitate the induction of rat bone mesenchymal stromal cells to differentiate into functional neurons. Cytotherapy. 2013;15:961-70.

[63] Engler AJ, Sen S, Sweeney HL, Discher DE. Matrix elasticity directs stem cell lineage specification. Cell. 2006;126:677-89.

[64] Benoit DS, Schwartz MP, Durney AR, Anseth KS. Small functional groups for controlled differentiation of hydrogel-encapsulated human mesenchymal stem cells. Nat Mater. 2008;7:816-23.

[65] Xia L, Lin K, Jiang X, Fang B, Xu Y, Liu J, et al. Effect of nano-structured bioceramic surface on osteogenic differentiation of adipose derived stem cells. Biomaterials. 2014;35:8514-27.

[66] Dulgar-Tulloch AJ, Bizios R, Siegel RW. Differentiation of human mesenchymal stem cells on nano- and micro-grain size titania. Materials Science and Engineering: C. 2011;31:357-62.

[67] Subramony SD, Su A, Yeager K, Lu HH. Combined effects of chemical priming and mechanical stimulation on mesenchymal stem cell differentiation on nanofiber scaffolds. Journal of Biomechanics. 2014;47:2189-96.

[68] Arahira T, Todo M. Effects of Proliferation and Differentiation of Mesenchymal Stem Cells on Compressive Mechanical Behavior of Collagen/ $\beta$-TCP Composite Scaffold. Journal of the Mechanical Behavior of Biomedical Materials. 2014;39:218-30.

[69] Faia-Torres AB, Guimond-Lischer S, Rottmar M, Charnley M, Goren T, Maniura-Weber K, et al. Differential regulation of osteogenic differentiation of stem cells on surface roughness gradients. Biomaterials. 2014;35:902332. 


\section{Chapter 1}

[70] Hu X, Park S-H, Gil ES, Xia X-X, Weiss AS, Kaplan DL. The influence of elasticity and surface roughness on myogenic and osteogenic-differentiation of cells on silk-elastin biomaterials. Biomaterials. 2011;32:8979-89.

[71] Li J, Li G, Zhang K, Liao Y, Yang P, Maitz MF, et al. Co-culture of vascular endothelial cells and smooth muscle cells by hyaluronic acid micro-pattern on titanium surface. Applied Surface Science. 2013;273:24-31.

[72] Li J, Zhang K, Yang P, Liao Y, Wu L, Chen J, et al. Research of smooth muscle cells response to fluid flow shear stress by hyaluronic acid micro-pattern on a titanium surface. Experimental Cell Research. 2013;319:266372.

[73] Li J-a, Yang P, Zhang K, Ren H-I, Huang N. Preparation of SiO2/TiO2 and TiO2/TiO2 micropattern and their effects on platelet adhesion and endothelial cell regulation. Nuclear Instruments and Methods in Physics Research Section B: Beam Interactions with Materials and Atoms. 2013;307:575-9.

[74] Zhou Z, Chen J, Xiang L-j, Xu Y, Yang P, Li J-a, et al. Fabrication of 3D TiO2 micromesh on silicon surface and its effects on platelet adhesion. Materials Letters. 2014;132:149-52.

[75] Li H, Wen F, Wong YS, Boey FYC, Subbu VS, Leong DT, et al. Direct laser machining-induced topographic pattern promotes up-regulation of myogenic markers in human mesenchymal stem cells. Acta Biomaterialia. 2012;8:531-9

[76] Li Z, Gong Y, Sun S, Du Y, Lü D, Liu X, et al. Differential regulation of stiffness, topography, and dimension of substrates in rat mesenchymal stem cells. Biomaterials. 2013;34:7616-25.

[77] Nakamoto T, Wang X, Kawazoe N, Chen G. Influence of micropattern width on differentiation of human mesenchymal stem cells to vascular smooth muscle cells. Colloids and Surfaces B: Biointerfaces. 2014;122:31623.

[78] Moroni L, Licht R, de Boer J, de Wijn JR, van Blitterswijk CA. Fiber diameter and texture of electrospun PEOT/PBT scaffolds influence human mesenchymal stem cell proliferation and morphology, and the release of incorporated compounds. Biomaterials. 2006;27:4911-22.

[79] Yang N, Yang MK, Bi SX, Chen L, Zhu ZY, Gao YT, et al. Cells behaviors and genotoxicity on topological surface. Materials Science and Engineering: C. 2013;33:3465-73.

[80] Gao L, McBeath R, Chen CS. Stem Cell Shape Regulates a Chondrogenic Versus Myogenic Fate Through Rac1 and N-Cadherin. Stem Cells. 2010;28:564-72.

[81] Kilian KA, Bugarija B, Lahn BT, Mrksich M. Geometric cues for directing the differentiation of mesenchymal stem cells. Proceedings of the National Academy of Sciences of the United States of America. 2010;107:4872-7.

[82] McBeath R, Pirone DM, Nelson CM, Bhadriraju K, Chen CS. Cell shape, cytoskeletal tension, and RhoA regulate stem cell lineage commitment. Developmental cell. 2004;6:483-95.

[83] Dalby MJ, McCloy D, Robertson M, Agheli H, Sutherland D, Affrossman S, et al. Osteoprogenitor response to semi-ordered and random nanotopographies. Biomaterials. 2006;27:2980-7.

[84] McMurray RJ, Gadegaard N, Tsimbouri PM, Burgess KV, McNamara LE, Tare R, et al. Nanoscale surfaces for the long-term maintenance of mesenchymal stem cell phenotype and multipotency. Nat Mater. 2011;10:63744.

[85] Brammer KS, Choi C, Frandsen CJ, Oh S, Jin S. Hydrophobic nanopillars initiate mesenchymal stem cell aggregation and osteo-differentiation. Acta Biomaterialia. 2011;7:683-90.

[86] Popat KC, Leoni L, Grimes CA, Desai TA. Influence of engineered titania nanotubular surfaces on bone cells. Biomaterials. 2007;28:3188-97.

[87] Tsimbouri PM, Murawski K, Hamilton G, Herzyk P, Oreffo RO, Gadegaard N, et al. A genomics approach in determining nanotopographical effects on MSC phenotype. Biomaterials. 2013;34:2177-84.

[88] Zhao L, Liu L, Wu Z, Zhang Y, Chu PK. Effects of micropitted/nanotubular titania topographies on bone mesenchymal stem cell osteogenic differentiation. Biomaterials. 2012;33:2629-41.

[89] Oh S, Brammer KS, Li Y, Teng D, Engler AJ, Chien S, et al. Stem cell fate dictated solely by altered nanotube dimension. Proceedings of the National Academy of Sciences of the United States of America. 2009;106:2130.

[90] Khang D, Choi J, Im Y-M, Kim Y-J, Jang J-H, Kang SS, et al. Role of subnano-, nano- and submicron-surface features on osteoblast differentiation of bone marrow mesenchymal stem cells. Biomaterials. 2012;33:5997-6007.

[91] Dulgar-Tulloch A, Bizios R, Siegel R. Human mesenchymal stem cell adhesion and proliferation in response to ceramic chemistry and nanoscale topography. Journal of Biomedical Materials Research Part A. 2009;90:58694.

[92] Chiang C-Y, Chiou S-H, Yang W-E, Hsu M-L, Yung M-C, Tsai M-L, et al. Formation of TiO 2 nano-network on titanium surface increases the human cell growth. Dental Materials. 2009;25:1022-9. 
[93] Bucci-Sabattini V, Cassinelli C, Coelho PG, Minnici A, Trani A, Ehrenfest DMD. Effect of titanium implant surface nanoroughness and calcium phosphate low impregnation on bone cell activity in vitro. Oral Surgery, Oral Medicine, Oral Pathology, Oral Radiology, and Endodontology. 2010;109:217-24.

[94] Mendonça G, Mendonça DB, Simoes LG, Araujo AL, Leite ER, Duarte WR, et al. The effects of implant surface nanoscale features on osteoblast-specific gene expression. Biomaterials. 2009;30:4053-62.

[95] Wall I, Donos N, Carlqvist K, Jones F, Brett P. Modified titanium surfaces promote accelerated osteogenic differentiation of mesenchymal stromal cells in vitro. Bone. 2009;45:17-26.

[96] Qazi TH, Rai R, Boccaccini AR. Tissue engineering of electrically responsive tissues using polyaniline based polymers: A review. Biomaterials. 2014;35:9068-86.

[97] Beningo KA, Lo C-M, Wang Y-L. Flexible polyacrylamide substrata for the analysis of mechanical interactions at cell-substratum adhesions. Methods in cell biology. 2002;69:325-39.

[98] Fu J, Wang YK, Yang MT, Desai RA, Yu X, Liu Z, et al. Mechanical regulation of cell function with geometrically modulated elastomeric substrates. Nature methods. 2010;7:733-6.

[99] Discher DE, Mooney DJ, Zandstra PW. Growth factors, matrices, and forces combine and control stem cells. Science. 2009;324:1673-7.

[100] Her GJ, Wu H-C, Chen M-H, Chen M-Y, Chang S-C, Wang T-W. Control of three-dimensional substrate stiffness to manipulate mesenchymal stem cell fate toward neuronal or glial lineages. Acta Biomaterialia. 2013;9:5170-80.

[101] Habibovic P, Van der Valk C, Van Blitterswijk C, De Groot K, Meijer G. Influence of octacalcium phosphate coating on osteoinductive properties of biomaterials. Journal of materials science Materials in medicine. 2004;15:373-80.

[102] Davison N, Luo X, Schoenmaker T, Everts V, Yuan H, Barrère-de Groot F, et al. Submicron-scale surface architecture of tricalcium phosphate directs osteogenesis in vitro and in vivo. European cells \& materials. 2013;27:281-97.

[103] Zhang J, Luo X, Barbieri D, Barradas A, de Bruijn JD, van Blitterswijk CA, et al. The size of surface microstructures as an osteogenic factor in calcium phosphate ceramics. Acta biomaterialia. 2014;10:3254-63.

[104] Barradas AM, Fernandes HA, Groen N, Chai YC, Schrooten J, van de Peppel J, et al. A calcium-induced signaling cascade leading to osteogenic differentiation of human bone marrow-derived mesenchymal stromal cells. Biomaterials. 2012;33:3205-15.

[105] Yasko AW, Lane J, Fellinger E, Rosen V, Wozney J, Wang E. The healing of segmental bone defects, induced by recombinant human bone morphogenetic protein (rhBMP-2). A radiographic, histological, and biomechanical study in rats. The Journal of bone and joint surgery American volume. 1992;74:659. 



\section{Chapter 2}

\section{Dimension of surface microstructure as an osteogenic factor in calcium phosphate ceramics}

Jingwei Zhang ${ }^{a, b}$, Xiaoman Luo ${ }^{c, d}$, Davide Barbieri ${ }^{d}$, Ana M.C. Barradas ${ }^{e}$, Joost D. de Bruijn $^{c, d, f}$, Clemens A. van Blitterswijk ${ }^{\mathrm{a}, \mathrm{g}}$, Huipin Yuan ${ }^{\mathrm{a}, \mathrm{d}, \mathrm{g}, \mathrm{h}}{ }^{*}$

a Department of Tissue Regeneration, MIRA Institute for Biomedical Technology and Technical Medicine, University of Twente, P.O. Box 217, 7500AE Enschede, The Netherlands

${ }^{b}$ Key Laboratory of Advanced Technologies of Materials, Ministry of Education, School of Materials Science and Engineering, Southwest Jiaotong University, Chengdu, P.R. China

c Department of Biomaterials Science and Technology, MIRA Institute for Biomedical Technology and Technical Medicine, University of Twente, P.O. Box 217, 7500AE Enschede, The Netherlands

${ }^{d}$ Xpand Biotechnology BV, Bilthoven, The Netherlands

e Department of Medical Cell Biophysics, MIRA Institute for Biomedical Technology and Technical Medicine, University of Twente, P.O. Box 217, 7500AE Enschede, The Netherlands

${ }^{f}$ School of Engineering ans Materials Science(SEMS), Queen Mary University of London, E1 4NS London, United Kingdom

$g$ Complex Tissue Regeneration department, MERLN Institute for Technology Inspired Regenerative Medicine, Maastricht University, Universiteitsingel 40, 6229 ER, The Netherlands

${ }^{h}$ College of Physical Science and Technology, Sichuan University, Chengdu, China. 


\begin{abstract}
Microporosity of calcium phosphate (CaP) ceramics has shown its essential role in osteoinduction by $\mathrm{CaP}$ ceramics after ectopic implantation. Here we show that not the microporosity but the dimension of surface microstructural features is likely the osteogenic factor. Two tricalcium phosphate ceramics (TCP), namely TCP-S and TCP-B, were fabricated with equivalent chemistry and similar microporosity but different size in surface microstructural features. TCP-S has a grain size of $0.99 \pm 0.20 \mu \mathrm{m}$ and a micropore size of $0.65 \pm 0.25 \mu \mathrm{m}$, while TCP-B displays a grain size of $3.08 \pm 0.52 \mu \mathrm{m}$ and a micropore size of $1.58 \pm 0.65 \mu \mathrm{m}$. In vitro, both cell proliferation and osteogenic differentiation were significantly enhanced when human bone marrow stromal cells (hBMSCs) were cultured on TCP-S, without any osteogenic growth factors, as compared to TCP-B ceramic granules. Besides this, the possible involvement of a direct contact between cells and the TCP ceramic surface in osteogenic differentiation is shown with a trans-well culture model. When the ceramic granules were implanted in para-spinal muscle of dogs for 12 weeks, abundant bone was formed in TCP-S ( $21 \pm 10 \%$ bone in the available space), while no bone was formed in any of the TCP-B implants. The current in vitro and in vivo data reveal that the readily controllable cue, i.e. the dimension of surface microstructure, could be sufficient to induce osteogenic differentiation of mesenchymal stromal cells (MSCs) finally leading to ectopic bone formation in CaP ceramics.
\end{abstract}

\title{
Keywords:
}

Calcium phosphate, Microstructure, Surface topography, Mesenchymal stromal cell, Osteogenesis 


\subsection{Introduction}

Reconstruction of massive and segmental bone defects frequently arises from skeletal disease, congenital malformations, infection, trauma and post-cancer ablative surgery [1]. Currently, autografts, allografts and synthetic materials are considered for bone regeneration [2-4]. For decades autogenous bone graft has been the gold standard for bone repair, however the amount of harvestable autografts is limited. Allograft, which provides much larger tissue amount than autograft, may carry the unpredictable risks of immunological reaction and disease transmission [5]. Therefore more and more attention has been paid on developing synthetic materials with similar performances to autologous bone.

CaP ceramics are nowadays widely used as bone graft substitutes because their chemical composition resembles that of bone mineral and they have excellent biocompatibility and bioactivity [6-8]. Repair of non-critical-sized bone defects could be achieved with osteoconductive CaP ceramics, which could lead to bone ingrowth from the surrounding host bone bed. However, to repair critical-sized bone defects osteoinductive materials that could induce mesenchymal stem cells (MSCs) to form bone (i.e. after an ectopic implantation) are necessary. A specific group of CaP ceramics has been reported to be osteoinductive in several animal models after heterotopic/ectopic implantation of the materials (e.g. subcutaneously or intramuscularly) [9]. It has also been shown that materials with higher osteoinductive potential could heal the non critical-sized defect faster $[10,11]$. Furthermore, osteoinductive CaP ceramics could perform similarly to the autologous bone graft and recombinant human Bone Morphogenetic Protein 2 (rhBMP-2) in repairing critical-sized bone defects [12].

Osteoinduction of CaP ceramics is material-dependent. Among chemical composition [11-13], macrostructural properties $[9,14]$ and microporosity (i.e. the volume percentage of pores smaller than $10 \mu \mathrm{m}$ ) [15-17], the microporosity has shown its great importance for osteoinduction. Previous studies have shown that microporous hydroxyapatite (HA) ceramics could induce bone formation after subcutaneous [18] and intramuscular [19] implantations in dogs, while no bone formed in those materials lacking of microporosity. It 


\section{Chapter 2}

has been also reported that the osteoinductive potential of CaP ceramics increased with increasing microporosity $[12,20]$.

It has been generally suggested, but not demonstrated, that the presence of micropores in $\mathrm{CaP}$ ceramics increases the surface area enhancing the local concentration of growth factors including osteoinductive bone morphogenetic proteins (BMPs), which may induce osteogenic differentiation of MSCs to form bone [12, 19-21]. If the surface area, thus protein adsorption, plays roles in osteoinduction by $\mathrm{CaP}$ ceramics, changing the microstructural properties via altering the dimension of micropores would change their osteoinductive potential.

Next to the possible role of protein adsorption in osteogenic differentiation of MSCs, an increasing number of researches showed that MSCs could also respond to extracellular matrix signals via topographical cues to go towards osteogenic differentiation [22-24]. The change of microstructural features' dimensions alters surface properties which, at a certain scale and with specific topographies, may initiate osteogenic differentiation of stem cells [25]. In addition, the possible role of ion release from CaP ceramics in osteogenic differentiation of MSCs could not be ignored. The resulting change of the surface area will affect ion exchanges (e.g. Ca ion) with the surroundings finally influencing osteogenic response of the cells [26].

Taking together the possible roles of protein adsorption, surface topography and ion exchange in osteogenic differentiation of MSCs, we hypothesized that altering surface microstructure dimension will influence the osteoinduction potential of CaP ceramics. To test this hypothesis, we prepared two TCP ceramics (TCP-S and TCP-B) with similar microporosity but various micropore sizes and evaluated their osteoinductive potential in vitro with human bone marrow MSCs (hBMSCs) and in vivo in a canine ectopic model. 


\subsection{Materials and methods}

\subsubsection{CaP ceramics}

\subsubsection{Preparation of TCP ceramics with various microstructure dimensions}

CaP powders were synthesized by mixing Ca hydroxide (Fluka) and phosphoric acid (Fluka) at a $\mathrm{Ca} / \mathrm{P}$ ratio of 1.50 . TCP powders allowing large (TCP-B) or small (TCP-S) grains in final ceramics were prepared by controlling the reaction rates. $\mathrm{H}_{3} \mathrm{PO}_{4}$ solution was directly pooled into $\mathrm{Ca}(\mathrm{OH})_{2}$ suspension to get TCP-B, while $\mathrm{H}_{3} \mathrm{PO}_{4}$ solution was dropwise added into $\mathrm{Ca}(\mathrm{OH})_{2}$ suspension to get TCP-S. The powders were then foamed with diluted $\mathrm{H}_{2} \mathrm{O}_{2}(1 \%)$ and porogen (i.e. wax particles) to obtain porous green bodies. TCP-S ceramic was obtained by sintering the porous green bodies at $1050^{\circ} \mathrm{C}$ for 8 hours as the target, while TCP-B porous green bodies were sintered at different temperatures of $1050^{\circ} \mathrm{C}, 1100^{\circ} \mathrm{C}$ and $1150^{\circ} \mathrm{C}$ for 8 hours in order to get target TCP-B ceramic having porosities (both total porosity and microporosity) close to those of TCP-S, as measured with mercury intrusion porosimetry (MP; Auto Pore IV 9500, Micromeritics, Monchengladbach, Germany).

The TCP-S and TCP-B granules with size of 1-2 $\mathrm{mm}$ were prepared from the sintered porous ceramic bodies, ultrasonically cleaned first with acetone, then $70 \%$ ethanol and finally demineralized water. The granules were then dried at $80^{\circ} \mathrm{C}$ and finally sterilized with gamma irradiation (25 kGy, Isotron, Netherland BV, Ede, The Netherlands).

\subsubsection{Physico-chemical properties of TCP ceramics}

Chemical composition and crystal structure of the TCP granules were determined with X-ray diffraction (XRD, Rigaku, Tokyo, Japan). Grain and micropore size were studied with environmental scanning electron microscope (ESEM; XL30, ESEMFEG, Philips, Eindhoven, The Netherlands) in the secondary electron mode and quantified with ImageJ (v1.43u, NIH, USA). Grains and micropores were randomly selected from an image and the vertical lengths crossing the centre of each grain and micropore were considered as grain and pore size respectively. Data of total porosity (i.e. the volume percentage of pores), microporosity and micropore size distribution of the porous ceramics were obtained with mercury intrusion. The specific surface area was analyzed with the Brunauer, Emmett and Teller method (BET, ChemBET monopoint Physisorption, QuanTachrome GmbH, Germany). 


\subsubsection{Protein adsorption of the ceramics}

To demonstrate the influence of microstructure dimension on the protein adsorption, 100 $\mathrm{mg}$ of TCP-S and TCP-B granules were immersed in $2 \mathrm{~mL}$ basic culture medium (BM), consisting of Minimal Essential Medium-alpha ( $\alpha$-MEM, Gibco) supplemented with $10 \%$ fetal bovine serum (FBS, Lonza), $100 \mathrm{U} / \mathrm{mL}$ penicillin and $100 \mu \mathrm{g} / \mathrm{mL}$ streptomycin (Gibco), $2 \mathrm{mM}$ L-Glutamine (Gibco) and $0.2 \mathrm{mM}$ L-ascorbic acid 2-phosphate (ASAP, Sigma-Aldrich) and incubated at $37^{\circ} \mathrm{C}$ in humid atmosphere with $5 \% \mathrm{CO}_{2}$ for 1 and 4 days. After washing three times with PBS, $0.2 \mathrm{~mL}$ of RIPA buffer (Thermo scientific) was added to each sample and kept at $4^{\circ} \mathrm{C}$ for 10 minutes. Thereafter the amount of protein detached from the ceramics into RIPA buffer was measured with the QuantiPro ${ }^{\mathrm{TM}}$ BCA Assay Kit (Sigma, The Netherlands) following the manufacturer's instructions and fluorescence was measured with a spectrophotometer (Anthos Zenyth 3100, Anthos Labtec Instruments GmbH, Salzburg, Austria) at $620 \mathrm{~nm}$. A calibration curve was prepared using standard BSA solutions. Three samples were used per material $(n=3)$ and the protein content was expressed as mean $\pm S D$.

\subsubsection{Ion release from the ceramics}

To evaluate the influence of microstructure dimension on the ion release, $100 \mathrm{mg}$ of the TCP granules were immersed in $2 \mathrm{~mL}$ of $\mathrm{BM}$ and incubated at $37^{\circ} \mathrm{C}$ in humid atmosphere with $5 \%$ $\mathrm{CO}_{2}$ for 1, 4, 7 and 14 days. Ca concentration in the culture medium was measured with QuantiChrom $^{\mathrm{TM}} \mathrm{Ca}$ assay kit (BioAssay, USA) and compared to the control (i.e. BM without samples) following the manufacturer's guidelines. Absorbance measurements were performed with spectrophotometer at $620 \mathrm{~nm}$. Calcium concentration was expressed as mean $\pm S D$, measured through standard calibration curves. Three samples were used per material $(n=3)$. Meanwhile the ceramic particles were collected and subjected to XRD analysis and SEM evaluation of possible surface changes.

\subsubsection{Cell culture}

\subsubsection{Isolation and expansion of $h B M S C s$}

Bone marrow aspirates $(5-20 \mathrm{~mL}$ ) were obtained from a donor (69 years old, female) with written informed consent. In brief, aspirate was resuspended using $20 \mathrm{G}$ needles, plated at a density of $5 \times 10^{5}$ cells $/ \mathrm{cm}^{2}$ and cultured in proliferation medium containing BM and basic 
fibroblasts growth factor (bFGF, Instruchemie, the Netherlands, $1 \mathrm{ng} / \mathrm{mL}$ ). Cells were expanded at $37^{\circ} \mathrm{C}$ in a humid atmosphere with $5 \% \mathrm{CO}_{2}$, medium was refreshed twice a week and cells were subcultured until $80 \%$ confluence.

\subsubsection{Cell culture on TCP ceramics}

To study the influence of microstructure dimension on proliferation, osteogenic differentiation and bone related gene expression, hBMSCs were cultured on the TCP granules. $100 \mathrm{mg}$ TCP-S and TCP-B granules were soaked in BM for at least 4 hours before cell seeding. The TCP granules were placed in squared wells of polystyrene plates. A cell suspension of $150 \mu \mathrm{L} \mathrm{hBMSC}$ at passage 3 was seeded on the materials at a seeding density of 3,750,000 cells $/ \mathrm{mL}$. Cells were allowed to attach for 4 hours after which $2 \mathrm{~mL}$ BM were slowly added to each sample and the medium was refreshed every 3 days $(2 \mathrm{~mL})$. For stereomicroscopic observation and cell proliferation assay, samples were harvested at day 1 , 7, 14 and 21; for osteogenic differentiation assay, samples were harvested at day 7, 14 and 21; for bone related gene expression, samples were harvested at day 4,7 and 14; for BMP-2 production, samples were harvested at day 2 and 4 .

\subsubsection{Trans-well culture}

One hundred microgram TCP-S and TCP-B granules were loaded on the trans-well membrane fitting to 24-well plate and soaked in BM for at least 4 hours before cell seeding. Third passage hBMSCs were seeded at a density of 25,000 cells/well and cultured in BM under a 3day refreshment regime $(2 \mathrm{~mL})$. To evaluate cell proliferation and osteogenic differentiation, samples were harvested at day 1, 4, 7 and 14.

\subsubsection{Stereo microscopy}

Cell distribution on the granules was observed with a light microscope (LM; E600, Nikon, Japan) equipped with KL 1500 LCD camera after fixing with 10\% neutral formalin and staining with $1 \%$ methylene blue.

\subsubsection{Cell proliferation and osteogenic differentiation assays}

Cell proliferation was analyzed with a DNA assay (CyQuant Cell Proliferation Assay kit, Sigma, the Netherlands), while osteogenic differentiation was measured with an alkaline phosphatase (ALP) assay (CDP-star assay kit, Roche). Prior to the analyses, the samples were 
rinsed three times with PBS and stored at $-20^{\circ} \mathrm{C}$ until further use. One $\mathrm{mL}$ lysis buffer (prepared according to manufacturer's instructions of CyQuant Cell Proliferation Assay kit instructions) was added onto each sample, followed by two cycles of freezing and thawing at $-20^{\circ} \mathrm{C}$ and room temperature respectively.

After lysis, total DNA was measured by incubating $100 \mu \mathrm{L}$ lysed sample in the dark at room temperature for $1 \mathrm{~h}$. Afterwards, the samples were incubated with $100 \mu \mathrm{L}$ CyQuant GR dye at room temperature for 15 minutes. Fluorescence was measured using a spectrophotometer (Victor, Perkin Elmer) at an excitation of $480 \mathrm{~nm}$ and emission wave length of $520 \mathrm{~nm}$. The DNA content of cells was calculated through a standard DNA curve. Three samples were used per material $(n=3)$.

ALP activity was measured according to the manufacturer's instructions. Briefly, $40 \mu \mathrm{L}$ of CDP star substrate were incubated with $10 \mu \mathrm{L}$ cell lysate for $20 \mathrm{~min}$ after which luminescence was measured using a spectrophotometer (Victor, Perkin Elmer). ALP expression was normalized to DNA content. Three samples were used per material $(n=3)$.

\subsubsection{Bone-related gene expression}

Bone-related gene expression was evaluated with quantitative real-time polymerase chain reaction (PCR) assay. RNA isolation was performed using Trizol reagent (Invitrogen) and Nucleospin RNA isolation kit (Macherey-NagelGmbh\& Co.) according to the manufacturer's instructions. Total RNA was measured using a NanoDrop spectrophotometer (Nanodrop technologies, USA). The RNA was used to synthesize complementary DNA (cDNA) with iScriptcDNA Synthesis kit (BioRad) according to the manufacturer's instructions. PCR analysis was performed with the Bio-Rad real-time PCR system (Bio-Rad, Hercules, CA, USA) on markers of alkaline phosphatase (ALP), collagen type I (Col I), osteocalcin (OCN), Runtrelated transcription factor 2(runx2), bone morphogenetic protein 2 (BMP-2) and osteopontin (OPN), with beta-2 microglobulin (B2M) as the house-keeping gene for normalization. Primer sequences for ALP, Col I, OCN, runx2, BMP-2, OPN, and B2M are listed in Table 1. Data was analyzed using Bio-Rad iQ5 software. The relative amounts of target genes normalized by $B 2 M$ were calculated by $2^{-\Delta C T}$ method where $\Delta C_{T}=C_{T, T a r g e t}-C_{T, G A P D H}$. All experiments were done in triplicate $(n=3)$. 
Table 1. qPCR primer sequences

\begin{tabular}{lll}
\hline Gene & Forward primer & Reverse primer \\
\hline runx2 & ATGGCGGGTAACGATGAAAAT & ACGGCGGGGAAGACTGTGC \\
OCN & GGCAGCGAGGTAGTGAAGAG & GATGTGGTCAGCCAACTCGT \\
OPN & CCAAGTAAGTCCAACGAAAG & GGTGATGTCCTCGTCTGTA \\
ALP & ACAAGCACTCCCACTTCATC & TTCAGCTCGTACTGCATGTC \\
Col-I & AGGGCCAAGACGAAGACATC & AGATCACGTCATCGCACAACA \\
BMP-2 & GGTAGACCTGTATCGCAGGC & TTTTCCCACTCGTTTCTGGT \\
B2M & GACTTGTCTTTCAGCAAGGA & ACAAAGTCACATGGTTCACA \\
\hline
\end{tabular}

\subsubsection{ELISA assay of BMP-2}

The ELISA assay was used to quantify BMP-2 protein production. Samples were rinsed 3 times with PBS, and then $0.2 \mathrm{~mL}$ of RIPA buffer (Thermo scientific) was added to each sample and kept at $4{ }^{\circ} \mathrm{C}$ for 10 minutes and stored at $-80^{\circ} \mathrm{C}$ until further use. The total protein in the cell lysate was measured with the QuantiPro ${ }^{\mathrm{TM}}$ BCA Assay Kit (Sigma, The Netherlands) following the manufacturer's instructions. BMP-2 content in the cell lysate was measured using BMP-2 DuoSet ELISA Development kit (R\&D) according to the manufacturer's instructions. The BMP-2 content was normalized by total protein. All experiments were done in triplicates $(n=3)$.

\subsubsection{Ectopic bone formation}

To study the influence of microstructure dimension on osteoinduction, the ceramics were evaluated in an ectopic model in canine. Following the permission of the local animal care committee (Animal Center, Sichuan University, Chengdu, China), the TCP-S and TCP-B granules (1-2 mm, $1.0 \mathrm{cc}$ per implant) were implanted in the para-spinal muscles of 8 adult male dogs (mongrel, 10-15 kg) for 12 weeks. All surgeries were conducted under general anaesthesia by abdominal injection of sodium pentobarbital $(30 \mathrm{mg} / \mathrm{kg}$ body weight) and sterile condition. Following the surgeries, buprenorphine $(0.1 \mathrm{mg}$ per animal) was intramuscularly given to the animals as pain relief for 2 days, while penicillin ( $40 \mathrm{mg} / \mathrm{kg}$ ) was intramuscularly injected for 3 consecutive days to prevent infection. After operation, the 
animals were allowed for full weight bearing and received normal diet. At week 12 the dogs were sacrificed by a celiac injection of excessive amount of pentobarbital sodium. Implants were harvested with surrounding tissues and fixed in $4 \%$ formaldehyde, embedded in polymethyl methacrylate (PMMA) after a series of gradient ethanol dehydration. Nondecalcified sections were made by crossing the middle of the explants using a diamond saw (SP-1600, Leica, Germany) and stained with 1\% methylene blue (Sigma) and 0.3\% basic fuchsin (Sigma) solutions. Histological observation was performed using light microscopy. The histological slides were scanned with a scanner (DIMAGE Scan Elite5400 II, model AF5400-2, KONICA MINOLTA) for histomorphometrical analysis and bone formation was quantified as bone area normalised to the available space using Adobe Photoshop Elements 4.0 software.

\subsubsection{Statistical analysis}

Multiple comparisons were performed with two way analysis of variance (ANOVA) followed by Bonferroni post-test comparisons. $\mathrm{P}$ values $<0.05$ were considered as a statistically significant difference.

\subsection{Results}

\subsubsection{Physical-chemical characterization of TCP-B and TCP-S}

To get target TCP-B ceramic having porosities (both total porosity and microporosity) close to those of TCP-S, TCP-B porous green bodies were sintered at different temperatures of $1050^{\circ} \mathrm{C}, 1100^{\circ} \mathrm{C}$ or $1150^{\circ} \mathrm{C}$ for 8 hours. As expected from the literature [27], the porosity of TCP-B decreased with sintering temperature and TCP-B sintered at $1100^{\circ} \mathrm{C}$ having a microporosity close to that of TCP-S (sintered at $1050^{\circ} \mathrm{C}$ ). Thus TCP-S sintered at $1050^{\circ} \mathrm{C}$ and TCP-B sintered at $1100^{\circ} \mathrm{C}$ are the target ceramics subjected to further studies. The XRD patterns of TCP-S and TCP-B showed the two ceramics had the same chemistry (Figure 1A), being $\beta$-TCP. Different microstructure dimensions were observed with SEM (Figure 1B and 1C), showing smaller grains and smaller micropores in TCP-S than in TCP-B (Figure 1B, 1C). Quantitatively, TCP-S had a grain size of $0.99 \pm 0.20 \mu \mathrm{m}$ and a micropore size of $0.65 \pm 0.25 \mu \mathrm{m}$, while TCP-B displayed larger grain and micropore sizes, $3.08 \pm 0.52 \mu \mathrm{m}$ and $1.58 \pm 0.65 \mu \mathrm{m}$ respectively. The observed differences in micropore size were confirmed by mercury 
intrusion results (Figure 1D), which indicated a micropore range of $0.3-1.3 \mu \mathrm{m}$ with a peak at $0.55 \mu \mathrm{m}$ for TCP-S, while micropores in TCP-B ranged from 0.8 to $2.2 \mu \mathrm{m}$ with a peak at 1.58 $\mu \mathrm{m}$. BET analysis showed higher specific surface area $\left(1.2 \mathrm{~m}^{2} / \mathrm{g}\right)$ in TCP-S than in TCP-B $(0.8$ $\mathrm{m}^{2} / \mathrm{g}$ ). No difference in total porosity was seen with the mercury intrusion data, which resulted $70 \%$ and $72 \%$ for TCP-S and TCP-B respectively. Similar microporosity was obtained for TCP-S and TCP-B (i.e. 22\% and 23\% for TCP-S and TCP-B, respectively).
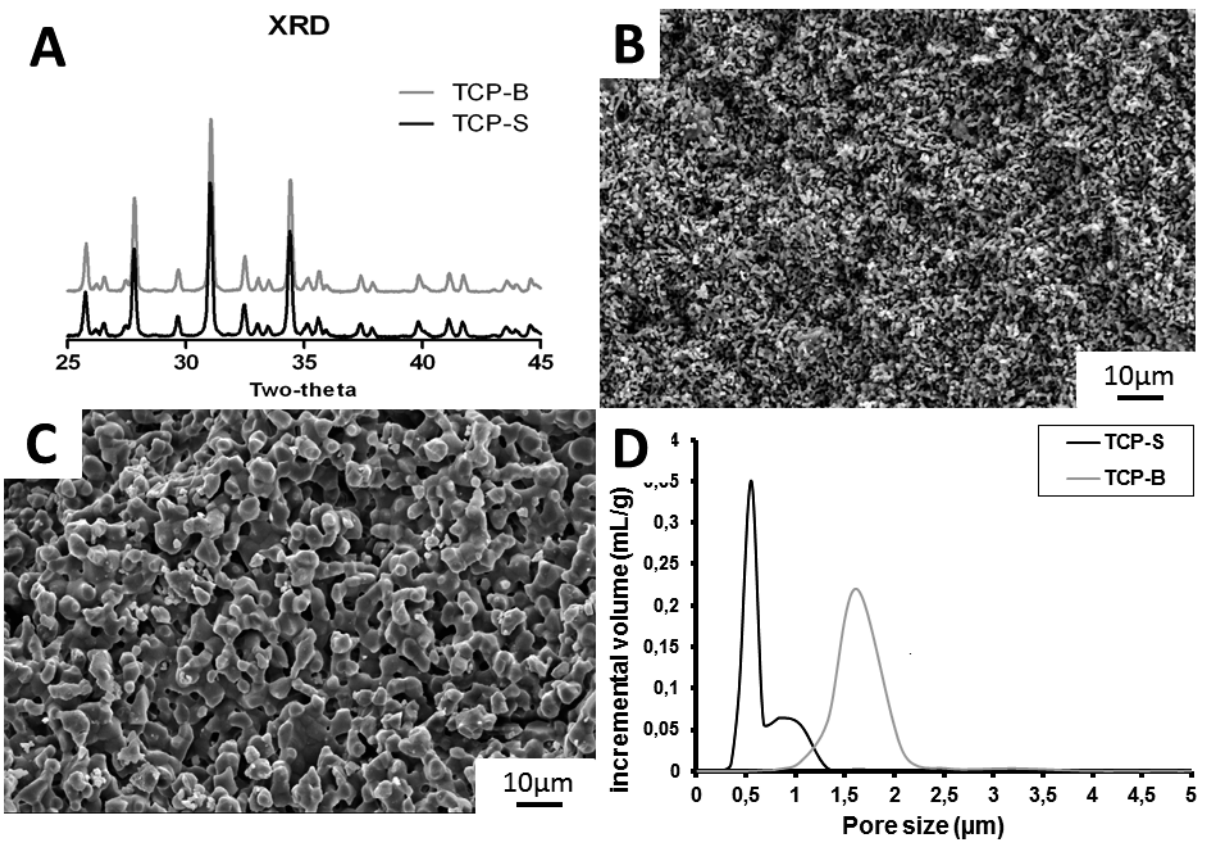

Figure 1. XRD pattern of the two ceramics (A), showing that TCP-S and TCP-B have the same chemistry; SEM observation of TCP-S $(B)$ and TCP-B $(C)$, showing the different microstructure dimension; Micropore distribution of TCP-S and TCP-B (D), where the former has smaller micropores than the latter.

\subsubsection{Protein adsorption and ion release}

As expected, TCP-S adsorbed higher amount of proteins than TCP-B at both day 1 and 4 (Figure 2A), since it has larger surface area. However, protein adsorption did not increase over time for both ceramics. Figure 2B depicts the results obtained on Ca concentration: in both TCP-S and TCP-B incubation solutions, the Ca concentration was lower compared to BM 
from day 1 to day 14 indicating that $\mathrm{Ca}$ was not released from the ceramics. A lower Ca concentration was observed in the case of TCP-S than TCP-B, indicating that TCP-S uptook higher amount of $\mathrm{Ca}$ ions than TCP-B.

A
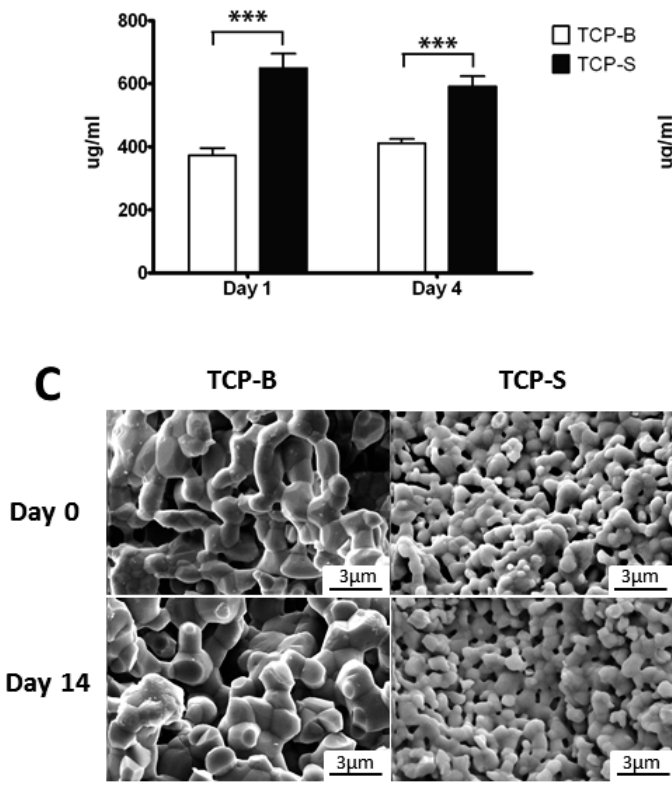

B $\mathrm{Ca}^{2+}$ amount in the medium

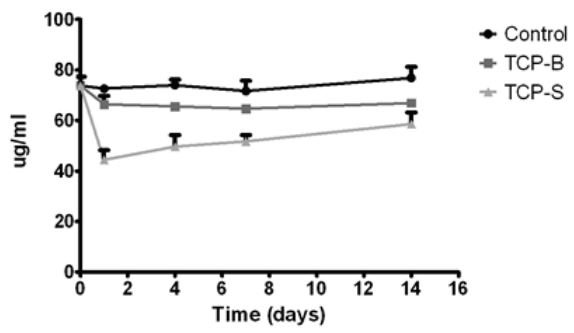

D
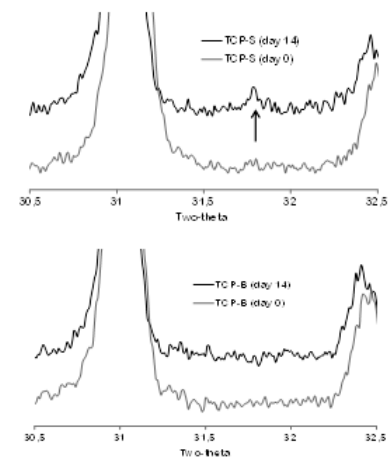

Figure 2. Protein adsorption of the ceramic granules (A), indicating that TCP-S has significantly higher protein adsorption potential than TCP-B; Calcium ion concentrations in culture medium (B), showing a significantly lower calcium concentration in mediums containing ceramic granules than in the control medium and an even significantly lower $C a$ concentration in the medium with TCP-S than with TCP-B (Statistically significant differences are marked by *** for $p<0.001)$; SEM observation of TCP-S and TCP-B before and after soaking in the culture medium for 14 days $(C)$, showing no surface change on either ceramic material after soaking; XRD pattern of TCP-S and TCP-B before and after soaking in the culture medium for 14 days (D), showing a new typical peak of apatite on TCP-S after a 14day soaking.

Surface changes were not noted for either TCP ceramics, i.e. no apatite layer formation was visible from SEM images on the ceramics' surface after 14 days (Figure 2C). XRD measurements on the TCP-S samples soaked for 14 days in BM revealed the formation of a new phase $(2 \theta=31.8$ degree, $d$-value $=2.814 \AA$ ), which is the typical peak of apatite-like CaP 
according to the international standard JCPDS 9-432 (Figure 2D). This specific XRD peak, or any others, could not be detected in any of TCP-B samples soaked in BM for two weeks (Figure 2B).

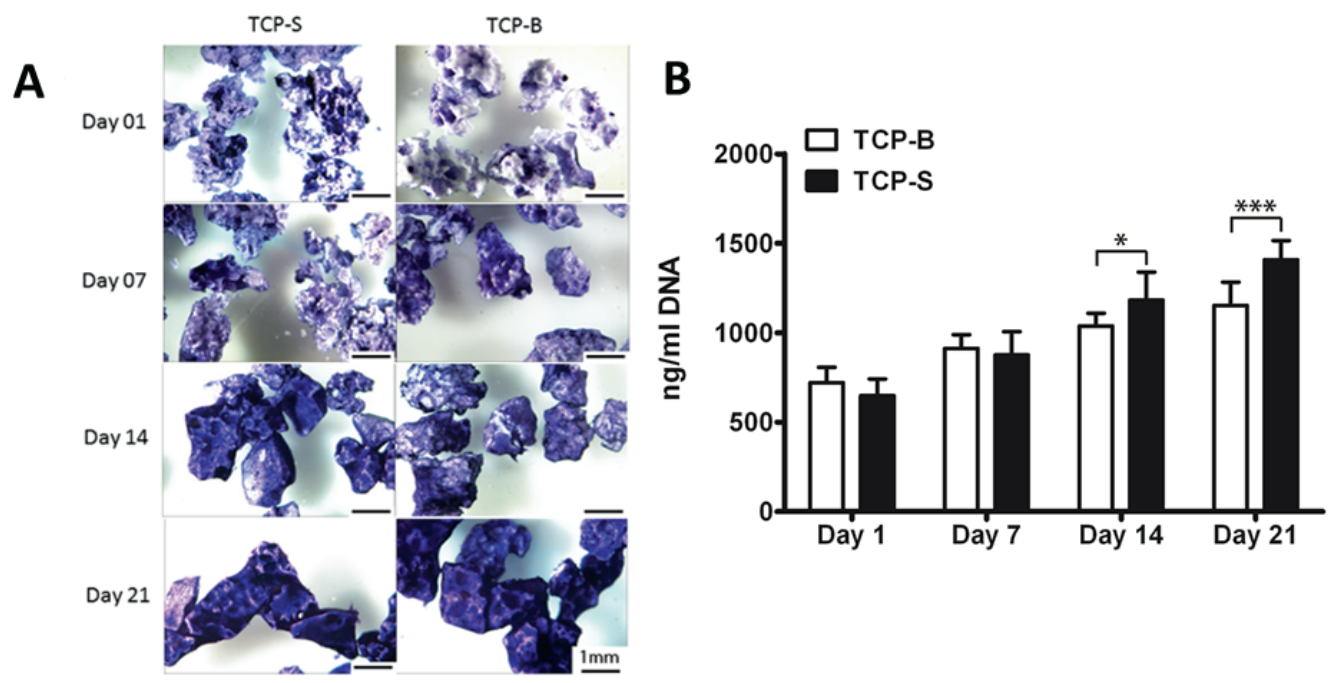

Figure 3. Stereomicroscopic observation (A) and DNA assay (B) of cells on TCP ceramics over time (Statistically significant differences are marked by * for $p<0.05$ and *** for $p<0.001$ ), showing a significantly greater proliferation of hBMSCS on TCP-S.

\subsection{3. hBMSCs attachment and proliferation}

To evaluate the distribution of hBMSCs on TCP-B and TCP-S surfaces, cells were cultured for 1, 7, 14 and 21 days and stained with methylene blue. As observed with stereo microscopy, hBMSCs were homogeneously distributed on both TCP-S and TCP-B granules 1 day after cell seeding and at later time points the staining was more intense (Fig. 3A). These results indicated that both TCP-S and TCP-B ceramics supported hBMSCs attachment, growth and proliferation. Cell proliferation of hBMSCs on TCP-B and TCP-S was further confirmed by DNA quantification (Fig. 3B), as the DNA amount increased from 1 to 21 days on both ceramics. There was no difference in DNA amounts between hBMSCs cultured on TCP-B and TCP-S at day 1 , indicating that the number of cells seeded on the two materials was the 
same. However, on day 14 and 21 more DNA was detected in TCP-S than TCP-B suggesting that TCP-S enhanced cell proliferation as compared to TCP-B.

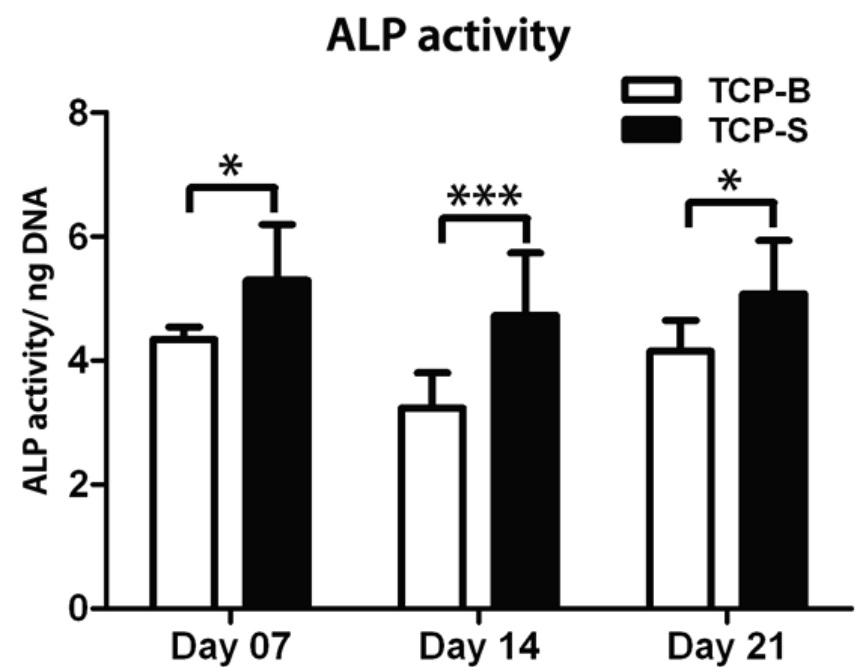

Figure 4. ALP activity (normalized to DNA) of hBMSCs cultured on TCP ceramic granules (Statistically significant differences are marked by * for $p<0.05$ and *** for $p<0.001$ ), showing an enhanced osteogenic differentiation of hBMSCS on TCP-S than on TCP-B granules.

\subsubsection{Osteogenic differentiation of hBMSCs on the ceramics}

The effect of microstructure dimension on osteogenic differentiation was evaluated by monitoring the activity of ALP (a marker of early osteogenesis). The specific ALP activity of hBMSCs cultured on TCP-S and TCP-B ceramics is shown in Figure 4, where ALP content of hBMSCs was normalized to DNA content over time. No changes in ALP activity of hBMSCs were observed over time on both TCP-S and TCP-B. However, the hBMSCS on TCP-S granules had significantly higher ALP activity at all the time points than TCP-B indicating an enhanced osteoblastic phenotype of hBMSCs on TCP-S.

\subsubsection{Bone-related gene expression}

Real time PCR was used to quantify the expression of osteoblastic genes of Col I, ALP, Runx-2, BMP-2, OPN and OCN (Fig. 5). No changes in the expressions of Runx2, ALP and Col I could be observed from day 7 to 14 on both materials. While gene expression of Runx 2 was slightly increased at day 7 and 14 as compared to day 4, ALP and Col I expressions were significantly 
elevated at day 7 and 14 than day 4 on both materials. Significant differences in gene expressions of Runx2, ALP and Col I were never seen between TCP-S and TCP-B at any time points tested. OCN gene expression increased with time on both materials and it was significantly higher on TCP-S at day 14 as compared on TCP-B. Similarly, OPN gene expression increased over time on TCP-S, while there was no markedly increase on TCP-B. OPN gene expression was significantly increased by TCP-S at both day 7 and 14. BMP-2 gene expression decreased from day 4 to 14 and it was significantly higher for TCP-B at day 4 . In line with the latter data, a higher BMP-2 protein level was also seen on TCP-B at day 4 (Figure. 6). The data obtained herein indicated that TCP-S enhanced osteocalcin and osteopontin gene expression whereas TCP-B enhanced BMP-2 gene expression and BMP-2 protein production in hBMSCs.

RUNX 2

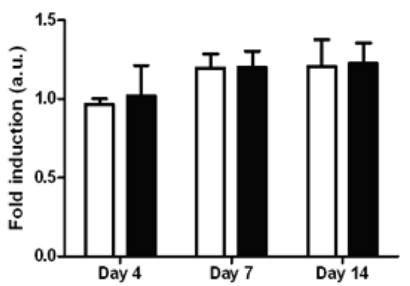

ALP

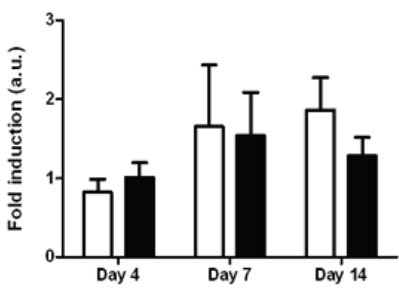

Osteocalcin

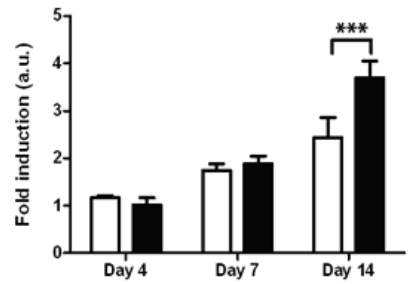

BMP-2

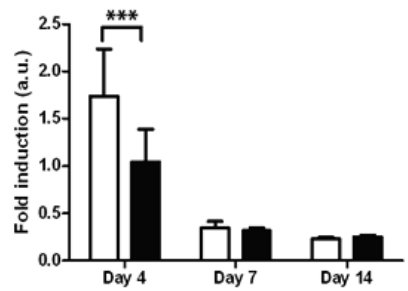

Osteopontin

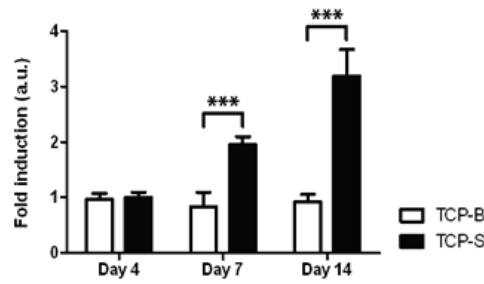

Collagen type I

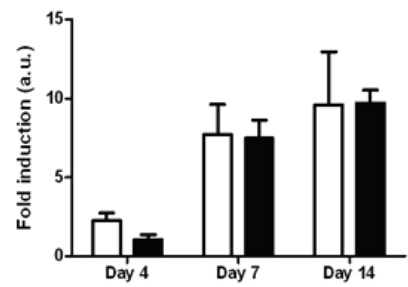

Figure 5. Osteogenic gene expression of hBMSCs cultured on the two ceramics (normalized to the B2M, statistically significant differences are marked by *** for $p<0.001)$.

\subsubsection{Proliferation and osteogenic differentiation of hBMSCs in trans-well culture}

To distinguish the role of surface topographical cue and chemical changes (e.g. ionic exchange) of the culture medium caused by the microstructure dimension, hBMSCs were cultured in trans-well plate with ceramics placed on the membrane. The proliferation and ALP activities of hBMSCs on the trans-well plates with both ceramics are shown in Figure 7. 
There was a slightly increasing trend in the cell number during 14 days of culture, while no differences were found in cell proliferation between the two conditions. The ALP activities of hBMSCs in both conditions increased from day 1 to 7 and decreased from day 7 to 14 . Importantly, the ALP activity of hBMSCs was significantly greater on the trans-well plates with TCP-B than with TCP-S at day 4 and 7.

\section{BMP-2/ total protein}

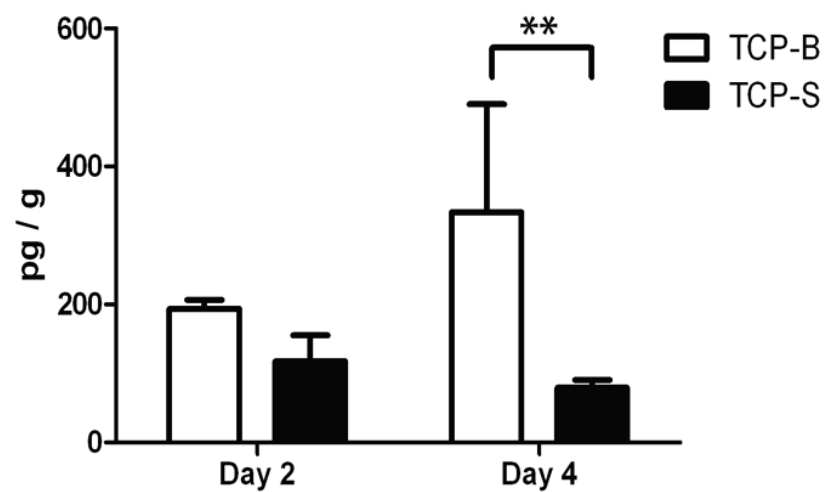

Figure 6. ELISA assay of BMP-2 produced by $h B M S C S$ cultured on ceramic granules (statistically significant difference is marked by ** for $P<0.01$ ), indicating a higher level of $B M P-2$ production in the case of TCP-B than in the case of on TCP-S after 4 days.

\subsubsection{Ectopic bone formation}

A total of 8 samples per TCP ceramics were harvested from 8 animals and were available for histological evaluation and histomorphometry. No bone formation occurred in any of TCP-B samples (Figure 8A). In contrast, abundant bone formation was formed in all TCP-S explants (Figure 8B). Closer observations showed the presence of bone inside and between the TCP-S granules (Figure 8D). The newly formed bone tissue consisting of mineralized bone matrix with osteocytes inside, with osteoblasts and osteoclasts on its surface (Figure 8D). Giant cells were observed in the TCP-S samples (Figure 8D) indicating a cell-mediated resorption of TCPS. At the same time, only soft tissues were observed in TCP-B and cell-mediated ceramic resorption was not often seen (Figure $8 C$ ). Quantitatively $21 \pm 10 \%$ of the available space in TCP-S explants was occupied by bone. 
A

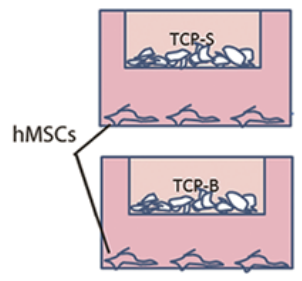

B

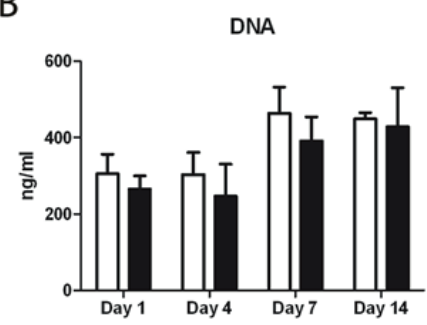

C

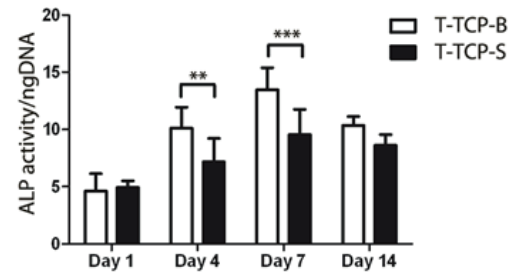

Figure 7. Proliferation and osteogenic differentiation of hBMSCs cultured in the trans-well plates. Schematic illustration of hBMSCs cultured in the trans-well plates $(A)$, where $h B M S C s$ were cultured on the bottom well of the trans-well plate with the ceramic granules on the membrane; hBMSCs proliferation increased over time in both conditions (B), but no significant difference was seen between TCP-S and TCP-B; ALP activity (normalized to DNA) significantly increased when hBMSCS was cultured in presence of TCP-B (C) (Statistically significant differences are marked by ** for $p<0.05$ and $* * *$ for $p<0.001$ ).

\subsection{Discussion}

Two TCP ceramics having equivalent chemistry and similar microporosity but different microstructure dimensions were prepared. As we hypothesized, the microstructural dimension has shown its role in determining the osteogenic potential of TCP ceramics both in vitro and in vivo. Osteogenic differentiation of hBMSC was enhanced on TCP-S (smaller microstructure dimension compared to TCP-B) both at protein (ALP activity) and gene (OCN and OPN were up-regulated) levels in vitro in the absence of osteogenic factors (e.g. dexamethasone) in the culture medium. In vivo, bone formation was found only in TCP-S and not in TCP-B in an osteoinduction canine model.

The ectopic bone formation induced by osteoinductive CaP ceramics suggested that the material could initiate osteogenic differentiation of MSCs. Recent data showed that BMSCs could be involved in the mechanism of osteoinduction by CaP ceramics [28]. It was shown that BMSCs could migrate from femoral bone to the bioceramic implants through the blood circulation, and contribute to the ectopic bone formation in a canine model. Moreover, it has been previously shown that hBMSCs display different cellular behavior according to the ceramic properties in vitro $[12,29]$. Therefore, examining the cellular behavior of hBMSCs 
cultured on TCP-S and TCP-B ceramics in vitro may provide insights on how MSCs respond to osteoinductive CaP ceramic in vivo to form bone.
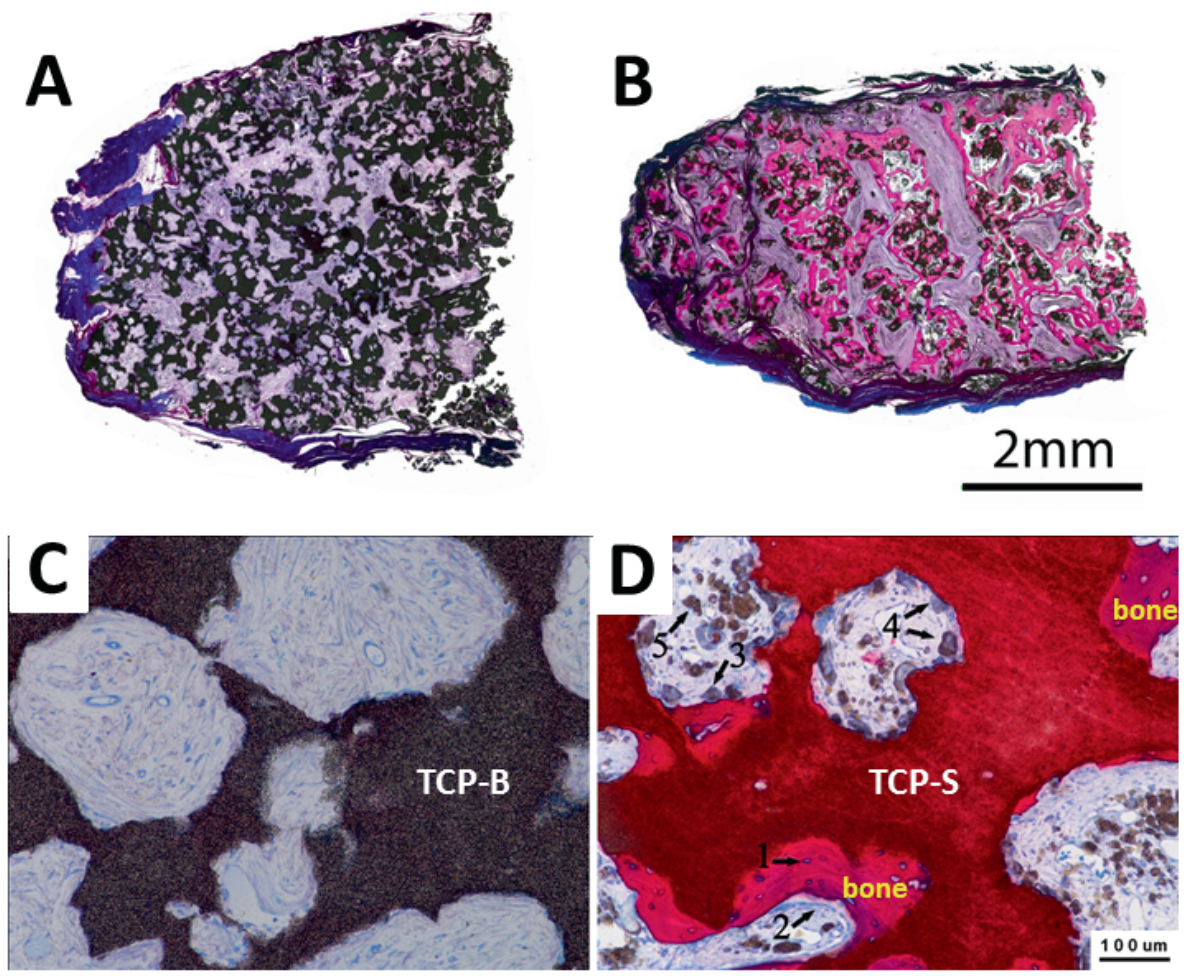

Figure 8. Histological overviews of TCP-B (A) and TCP-S (B) after a 12-week implantation in muscle of dog, showing bone formation in TCP-S and no bone in TCP-B; a high magnification image of TCP-B (C), showing the infiltration of fibrous tissue but absence of bone in TCP-B; $a$ high magnification image of TCP-S (D), showing the presence of mineralized bone matrix (bright pink), osteocytes (arrow 1), osteoblasts (arrow 2), osteoclast (arrow 3), giant cell (arrow 4) and ceramic debris (arrow 5) in TCP-S.

In vitro, the results of q-PCR analysis showed hBMSCs cultured on TCP-S granules exhibited higher expression of OCN at day 14 and OPN at day 7 and 14 (Figure 5). OCN is an established indicator of osteoblastic differentiation of hBMSCs and is synthesized only by mature osteoblasts, cementoblasts and odontoblasts to bind both collagen and $\mathrm{Ca}$ in the ECM of bone tissue [30]. OPN is highly expressed in bone and its expression is increased during osteoblastic differentiation of hBMSCs [26]. ALP is a cell surface glycoprotein, is the 
most widely recognized marker of osteoblastic differentiation. In this study, we used ALP/DNA to express the ALP activity per cell to characterize osteogenic differentiation corrected to cell numbers. The hBMSCs grown on the surface of TCP-S showed higher levels of ALP activity. Taken together, these results suggested that TCP-S induced hBMSCs to differentiate into osteogenic cells better than TCP-B without soluble osteogenic factors in the media.

In vivo, an intramuscular implantation in canine model was used to determine the osteoinductive potential of the two materials. Histologically, abundant newly formed bone was observed in TCP-S scaffolds but no bone formation occurred in TCP-B implants after 12 weeks. So far, the underlying mechanism for osteoinduction is not yet fully understood. However, some previously proposed mechanisms could help to explain in this study why the smaller microstructural dimension was effective at inducing osteogenic differentiation in vitro and bone formation in vivo.

Protein adsorption has long been suggested to play an important role in osteoinduction of CaP ceramics. BMPs (e.g. BMP-2) are dose-dependent bone forming growth factors [31]. It has been emphasized that CaP ceramics first concentrates BMPs from body fluids and then induce bone formation [7, 32]. In this study, TCP-S enhanced osteogenic differentiation of hBMSCs in absence of growth factors such as BMPs in the culture medium. However, the role of protein adsorption in osteoinduction could not be excluded since such growth factors do exist in vivo. Given the same microporosity, the decreased micropore size increased the surface area leading to improved protein adsorption of TCP-S (Figure 2A). hBMSCs may be sensitive not only to the adsorbed osteogenic growth factors, but also to non-osteogenic growth factors (e.g. fibronectin and vitronectin in serum) that also induce osteogenic differentiation via, for instance, a focal adhesion process which in turn could trigger osteogenic differentiation of stem cells via possibly mechanotransduction [22]. Next to this, with the presence of osteogenic growth factors in vivo, TCP-S would concentrate more growth factors in amount and thus further enhance osteogenic differentiation of MSCs infiltrating into the implants to form bone as compared to TCP-B. 
Several studies also showed the role of topographical cues in stem cell differentiation towards a desired direction for tissue regeneration and cell therapy [33-35]. Importantly, at certain scale and shape, nano- or micro-structures triggered osteogenic differentiation of hBMSCs in vitro without osteogenic factors (e.g. dexamethasone) in the culture medium [13]. With the same microporosity but with smaller microstructural dimension, TCP-S, compared to TCP-B, enhanced osteogenic differentiation of hBMSC. However, it is currently a challenge to distinguish the role of protein adsorption and topographic cue created by surface topography in biological responses of a material.

It is assumed that the $\mathrm{Ca}$ ion $\left(\mathrm{Ca}^{2+}\right)$ release from the materials is an important factor in inducing bone formation. In this study, it was expected that, with a larger surface area, TCP$\mathrm{S}$ would release higher amount of $\mathrm{Ca}^{2+}$ into the culture medium and thus enhance osteogenic differentiation of hBMSCs in vitro. Instead we observed a decrease in $\mathrm{Ca}^{2+}$ concentration with both ceramics (Figure $2 \mathrm{~B}$ ), suggesting uptake of $\mathrm{Ca}^{2+}$ from the culture medium. The decrease of $\mathrm{Ca}^{2+}$ in culture medium may result in precipitation of $\mathrm{Ca} / \mathrm{P}$ on the ceramic surface to form an apatite layer which would enhance the osteogenic differentiation of MSCs as assumed by literature $[17,36]$. We observed that the amount of $\mathrm{Ca}^{2+}$ in the medium with TCP-S was significantly lower than in the medium with TCP-B $(P<0.001)$ (Figure 2B). XRD measurements on the TCP-S samples soaked for 14 days in BM revealed the formation of a new phase, identified as a typical apatite-like CaP according to the international standard JCPDS 9-432 (Figure 2D). Such new peak indicates that Ca ions may have precipitated onto the surface of TCP-S leading to a slight increase in Ca/P ratio of TCP-S. However, this peak was not detectable in any of TCP-B samples soaked in BM 14 days, most likely due to the limited amount of Ca adsorbed by TCP-B from the BM (Figure 2B). However, apatite layer formation was not observed using SEM on the surface of both ceramics suggesting that the amount of $\mathrm{Ca}^{2+}$ adsorbed by the surface of the two materials could have been not sufficient to trigger visible apatite layer (figure $2 \mathrm{C}$ ). Our previous work showed that when soaking $\beta$-TCP in protein-free medium (i.e. $\alpha$-MEM alone), abundant precipitation of $\mathrm{Ca}$ in the form of apatite layer on its surface occurred and was visible at SEM. Conversely, no apatite layer formation happened when $\beta$-TCP was immersed in medium containing proteins (i.e. basic medium) [29], similarly to what we observed in this study. Other studies confirmed 
that increasing amounts of serum had increasing inhibition effects on the apatite layer formation onto biomaterials $[37,38]$.

In the trans-well plate culture, a higher ALP activity of hBMSCs was observed in the case of TCP-B. This result is in line with literature where a high extracellular $\mathrm{Ca}^{2+}$ concentration was reported to enhance osteogenic differentiation of hBMSCs [26]. It could well be that the higher local Ca concentration on TCP-S surface enhanced osteogenic differentiation of hBMSCs in this study when cells were cultured directly on TCP ceramics. Taken together, the results indicated that a direct contact of cells to the material's surface is necessary for osteogenic differentiation of MSC to occur in TCP-S. This indication was supported by the observation that ectopic bone formation induced by CaP ceramics always starts on the material's surface [39].

BMPs (e.g. BMP-2 and BMP-7) were detected in cells involved in bone morphogenesis initiated by osteoinductive CaP ceramics $[40,41]$ while, in this study, BMP-2 was more enhanced at both the gene and protein levels in hBMSCs cultured on TCP-B. With the protein production of BMP-2 by hBMSCs cultured on TCP-B, we may expect osteoinduction in TCP-B as well. However, since BMP induces bone formation in a dose-dependent manner [31], the amount of BMP-2 produced may not be enough to induce bone formation in the case of TCP-B indicated by the current result.

Being the main inorganic components of hard tissues, CaP ceramics are often considered as promising bone grafting materials because of their biocompatibility, bioactivity and resorbability. The influence of chemistry on the properties of CaP ceramics has been evaluated often. For instance, various studies on the influence of chemistry (e.g. the ratio of TCP to HA) on resorption rate allowed for the fabrication of CaP ceramics with desired resorption rates $[12,20,42]$. As histologically observed in the current study, the resorption rate differed between the two ceramics, with a clear cell-mediated resorption in TCP-S. The delayed resorption of TCP-B might be caused by its lower surface area, which limited the chemical dissolution of the ceramic [20]. It could also be because of the influence of surface topography on phagocytes involved in cell-mediated resorption of materials (e.g. macrophages) $[3,43]$. We observed that osteopontin was up-regulated in hBMSCs on TCP-S. 


\section{Chapter 2}

The important role osteopontin plays in anchoring osteoclasts to the extracellular matrix has been shown [44], therefore the cell-mediated resorption and osteoinduction of TCP-S may be coupled. Responding to the surface microstructure of TCP-S, hBMSCs may undergo osteogenic differentiation to form bone and, at the same time, produce osteopontin which induces osteoclast-like cells to resorb TCP-S. This finding may explain the previous observation that, although TCP is generally considered as resorbable material, it was not resorbed at all in some studies where TCP ceramics with large grains were used [45].

The current findings suggest that the small dimension of surface microstructure of TCP-S may provide a suitable local environment for osteoinduction to occur. It could concentrate ions to form apatite-like CaP and proteins (e.g. growth factors) or provide topographic cues, determining the spatial regulation of proliferation and osteogenic differentiation of MSCs on the surface. A careful design of materials to distinguish the role of ion release, protein adsorption and physical cues in osteoinduction of CaP ceramics is subjected to the goal of future studies.

The microstructure modification, previously by varying microporosity [46] and now by adjusting the microstructure dimension, could render CaP ceramics osteoinductive and more resorbable. The finding would encourage further optimization of CaP ceramics for bone regeneration in the challenging aim to ideally replace autologous bone in clinics.

\subsection{Conclusions}

Given the same microporosity and chemistry, the microstructural dimension affects the bone forming ability of TCP ceramics. A small microstructure is suggested as a favorite scale for ectopic bone formation in this study. hBMSCs are sensitive to the local environment created by the surface microstructure and undergo osteogenic differentiation in absence of osteogenic growth factors in vitro. Although the mechanism is not fully understood, the current data partially explain how MSCs may respond to surface microstructure to form bone. 


\section{References}

[1] Phillips J, Forrest C, Gruss J. Current concepts in the use of bone grafts in facial fractures. Basic science considerations. Clinics in plastic surgery. 1992;19:41-58.

[2] Lan Levengood SK, Polak SJ, Wheeler MB, Maki AJ, Clark SG, Jamison RD, et al. Multiscale osteointegration as a new paradigm for the design of calcium phosphate scaffolds for bone regeneration. Biomaterials. 2010;31:3552-63.

[3] Okuda T, loku K, Yonezawa I, Minagi H, Kawachi G, Gonda Y, et al. The effect of the microstructure of $\beta$ tricalcium phosphate on the metabolism of subsequently formed bone tissue. Biomaterials. 2007;28:2612-21.

[4] Faour O, Dimitriou R, Cousins CA, Giannoudis PV. The use of bone graft substitutes in large cancellous voids: Any specific needs? Injury. 2011;42:S87-S90.

[5] Burchardt H, Glowczewskie Jr F, Enneking W. The effect of adriamycin and methotrexate on the repair of segmental cortical autografts in dogs. J Bone Joint Surg Am. 1983;65:103-8.

[6] Damien CJ, Parsons JR. Bone graft and bone graft substitutes: a review of current technology and applications. Journal of Applied Biomaterials. 1991;2:187-208.

[7] Yang Z, Yuan H, Tong W, Zou P, Chen W, Zhang X. Osteogenesis in extraskeletally implanted porous calcium phosphate ceramics: variability among different kinds of animals. Biomaterials. 1996;17:2131-7.

[8] Bohner M, Gbureck U, Barralet J. Technological issues for the development of more efficient calcium phosphate bone cements: a critical assessment. Biomaterials. 2005;26:6423-9.

[9] Barradas AMC, Yuan H, van Blitterswijk CA, Habibovic P. Osteoinductive biomaterials: current knowledge of properties, experimental models and biological mechanisms. Eur Cell Mater. 2011;21:407-29.

[10] Habibovic P, Van der Valk C, Van Blitterswijk C, De Groot K, Meijer G. Influence of octacalcium phosphate coating on osteoinductive properties of biomaterials. Journal of Materials Science: Materials in Medicine. 2004;15:373-80.

[11] Yuan H, Van Blitterswijk C, De Groot K, De Bruijn J. A comparison of bone formation in biphasic calcium phosphate (BCP) and hydroxyapatite (HA) implanted in muscle and bone of dogs at different time periods. Journal of Biomedical Materials Research Part A. 2006;78:139-47.

[12] Yuan H, Fernandes H, Habibovic P, De Boer J, Barradas A, De Ruiter A, et al. Osteoinductive ceramics as a synthetic alternative to autologous bone grafting. Proceedings of the National Academy of Sciences. 2010;107:13614-9.

[13] Oh S, Brammer KS, Li Y, Teng D, Engler AJ, Chien S, et al. Stem cell fate dictated solely by altered nanotube dimension. Proceedings of the National Academy of Sciences. 2009;106:2130-35.

[14] Ripamonti U, Richter PW, Thomas ME. Self-inducing shape memory geometric cues embedded within smart hydroxyapatite-based biomimetic matrices. Plastic and reconstructive surgery. 2007;120:1796-1807.

[15] Curtis A, Wilkinson C. Nantotechniques and approaches in biotechnology. TRENDS in Biotechnology. 2001;19:97-101.

[16] Galli C, Piemontese M, Lumetti S, Manfredi E, Macaluso G, Passeri G. The importance of WNT pathways for bone metabolism and their regulation by implant topography. European Cells and Materials. 2012;24:46-59.

[17] Habibovic P, Sees TM, van den Doel MA, van Blitterswijk CA, de Groot K. Osteoinduction by biomaterialsphysicochemical and structural influences. Journal of Biomedical Materials Research Part A. 2006;77:747-62.

[18] Yamasaki H, Sakai H. Osteogenic response to porous hydroxyapatite ceramics under the skin of dogs. Biomaterials. 1992;13:308-12.

[19] Yuan H, Kurashina K, de Bruijn JD, Li Y, De Groot K, Zhang X. A preliminary study on osteoinduction of two kinds of calcium phosphate ceramics. Biomaterials. 1999;20:1799-806.

[20] Habibovic P, Yuan H, Van Der Valk CM, Meijer G, Van Blitterswijk CA, De Groot K. 3D microenvironment as essential element for osteoinduction by biomaterials. Biomaterials. 2005;26:3565-75.

[21] Urist MR, Huo YK, Brownell AG, Hohl WM, Buyske J, Lietze A, et al. Purification of bovine bone morphogenetic protein by hydroxyapatite chromatography. Proceedings of the National Academy of Sciences. 1984;81:371-5.

[22] Guilak F, Cohen DM, Estes BT, Gimble JM, Liedtke W, Chen CS. Control of stem cell fate by physical interactions with the extracellular matrix. Cell Stem Cell. 2009;5:17-26. 
[23] Fu J, Wang YK, Yang MT, Desai RA, Yu X, Liu Z, et al. Mechanical regulation of cell function with geometrically modulated elastomeric substrates. Nature methods. 2010;7:733-6.

[24] McBeath R, Pirone DM, Nelson CM, Bhadriraju K, Chen CS. Cell shape, cytoskeletal tension, and RhoA regulate stem cell lineage commitment. Developmental cell. 2004;6:483-95.

[25] Kilian KA, Bugarija B, Lahn BT, Mrksich M. Geometric cues for directing the differentiation of mesenchymal stem cells. Proceedings of the National Academy of Sciences. 2010;107:4872-7.

[26] Barradas A, Fernandes HA, Groen N, Chai YC, Schrooten J, van de Peppel J, et al. A calcium-induced signaling cascade leading to osteogenic differentiation of human bone marrow-derived mesenchymal stromal cells. Biomaterials. 2012;33:3205-15.

[27] Daculsi G, Aguado E, Goyenvalle E, LeGeros RZ, LeGeros JP, Grimandi G, et al. Effect of sintering process of HA/TCP bioceramics on microstructure, dissolution, cell proliferation and bone ingrowth. Key Engineering Materials. 2008;361:1139-42.

[28] Song G, Habibovic P, Bao C, Hu J, van Blitterswijk CA, Yuan H, et al. The homing of bone marrow MSCs to non-osseous sites for ectopic bone formation induced by osteoinductive calcium phosphate. Biomaterials. 2013;34:2167-76.

[29] Barradas AM, Monticone V, Hulsman M, Danoux C, Fernandes H, Tahmasebi Z, et al. Molecular mechanisms of biomaterial-driven osteogenic differentiation in human mesenchymal stromal cells. Integr Biol. 2013;5:920-31.

[30] Sun H, Wu C, Dai K, Chang J, Tang T. Proliferation and osteoblastic differentiation of human bone marrowderived stromal cells on akermanite-bioactive ceramics. Biomaterials. 2006;27:5651-7.

[31] Yasko AW, Lane J, Fellinger E, Rosen V, Wozney J, Wang E. The healing of segmental bone defects, induced by recombinant human bone morphogenetic protein (rhBMP-2). A radiographic, histological, and biomechanical study in rats. The Journal of bone and joint surgery American volume. 1992;74:659-70.

[32] Ripamonti U. Osteoinduction in porous hydroxyapatite implanted in heterotopic sites of different animal models. Biomaterials. 1996;17:31-5.

[33] Dalby MJ, Gadegaard N, Tare R, Andar A, Riehle MO, Herzyk P, et al. The control of human mesenchymal cell differentiation using nanoscale symmetry and disorder. Nature materials. 2007;6:997-1003.

[34] Gittens RA, McLachlan T, Olivares-Navarrete R, Cai Y, Berner S, Tannenbaum R, et al. The effects of combined micron-/submicron-scale surface roughness and nanoscale features on cell proliferation and differentiation. Biomaterials. 2011;32:3395-403

[35] Guvendiren M, Burdick JA. The control of stem cell morphology and differentiation by hydrogel surface wrinkles. Biomaterials. 2010;31:6511-8.

[36] Fujibayashi S, Neo M, Kim H-M, Kokubo T, Nakamura T. Osteoinduction of porous bioactive titanium metal. Biomaterials. 2004;25:443-50.

[37] Marques P, Serro A, Saramago B, Fernandes A, Magalhaes M, Correia R. Mineralisation of two phosphate ceramics in HBSS: role of albumin. Biomaterials. 2003;24:451-60.

[38] Garnett J, Dieppe P. The effects of serum and human albumin on calcium hydroxyapatite crystal growth. Biochem J. 1990;266:863-8.

[39] Yuan H, Yang Z, Li Y, Zhang X, De Bruijn J, De Groot K. Osteoinduction by calcium phosphate biomaterials. Journal of Materials Science: Materials in Medicine. 1998;9:723-6.

[40] Duneas N, Crooks J, Ripamonti U. Transforming growth factor- $\beta$ 1: induction of bone morphogenetic protein genes expression during endochondral bone formation in the baboon, and synergistic interaction with osteogenic protein-1 (BMP-7). Growth Factors. 1998;15:259-77.

[41] Ripamonti U, Heliotis M, Rueger D, Sampath T. Induction of cementogenesis by recombinant human osteogenic protein-1 (hOP-1/BMP-7) in the baboon (Papio ursinus). Archives of oral biology. 1996;41:121-6.

[42] Yamada S, Heymann D, Bouler J-M, Daculsi G. Osteoclastic resorption of calcium phosphate ceramics with different hydroxyapatite/ $\beta$-tricalcium phosphate ratios. Biomaterials. 1997;18:1037-41.

[43] Pirraco R, Reis R, Marques A. Effect of monocytes/macrophages on the early osteogenic differentiation of hBMSCs. Journal of Tissue Engineering and Regenerative Medicine. 2012; 7: 392-400.

[44] Merry K, Dodds R, Littlewood A, Gowen M. Expression of osteopontin mRNA by osteoclasts and osteoblasts in modelling adult human bone. Journal of cell science. 1993;104:1013-20. 
[45] Handschel J, Wiesmann HP, Stratmann U, Kleinheinz J, Meyer U, Joos U. TCP is hardly resorbed and not osteoconductive in a non-loading calvarial model. Biomaterials. 2002;23:1689-95.

[46] Campion CR, Chander C, Buckland T, Hing K. Increasing strut porosity in silicate-substituted calciumphosphate bone graft substitutes enhances osteogenesis. Journal of Biomedical Materials Research Part B: Applied Biomaterials.2011;97 



\section{Chapter 3}

\section{Surface structure of calcium phosphate ceramics instructs inductive bone formation via influencing on morphology and primary cilia structure of stem cells}

Jingwei Zhang ${ }^{\mathrm{a}, \mathrm{b}}$, Melis Dalbayc, Xiaoman Luo ${ }^{\mathrm{d}, \mathrm{e}}$, Erik Vrij $^{\mathrm{a}}{ }^{\text {, Davide Barbieri }}{ }^{\mathrm{d}}$, Lorenzo Moroni $^{\mathrm{a}, \mathrm{g}}$, Martin M. Knight ${ }^{c}$, Joost D. de Bruijn ${ }^{\mathrm{c}, \mathrm{d}, \mathrm{e}}$, Clemens A. van Blitterswijk ${ }^{\mathrm{a}, \mathrm{g}}$, Huipin Yuan $^{\mathrm{a}, \mathrm{d}, \mathrm{f}, \mathrm{g}^{*}}$

a Department of Tissue Regeneration, MIRA Institute for Biomedical Technology and Technical Medicine, University of Twente, P.O. Box 217, 7500AE Enschede, The Netherland

${ }^{b}$ Key Laboratory of Advanced Technologies of Materials, Ministry of Education, School of Materials Science and Engineering, Southwest Jiaotong University, Chengdu, P.R. China

' School of Engineering and Materials Science (SEMS), Queen Mary University of London, E1 4NS London, United Kingdom

${ }^{d}$ Xpand Biotechnology BV, Bilthoven, The Netherlands

e Department of Biomaterials Science and Technology, MIRA Institute for Biomedical Technology and Technical Medicine, University of Twente, P.O. Box 217, 7500AE Enschede, The Netherlands

${ }^{f}$ College of Physical Science and Technology, Sichuan University, Chengdu, China.

$g$ Complex Tissue Regeneration department, MERLN Institute for Technology Inspired Regenerative Medicine, Maastricht University, Universiteitsingel 40, 6229 ER, The Netherlands 


\title{
Chapter 3
}

\begin{abstract}
The surface structure of synthetic biomaterials is known to play an important role in osteogenesis. At the submicron scale, the surface structure of calcium phosphate (CaP) ceramics could instruct the osteogenic differentiation of stem cells in vitro and induce bone formation ectopically in vivo. In this study, we developed a 2D CaP ceramic system to elucidate how surface structure instructs the induction of bone formation. Two types of tricalcium phosphate ceramic discs (TCP), namely TCP-S (with a submicron-structured surface) and TCP-B (having a micron-scaled surface structure), were prepared for both in vitro and in vivo evaluations. Without any osteogenic growth factors in the culture medium, the osteogenic differentiation of human bone marrow stromal cells (hBMSCs) was more enhanced on TCP-S than on TCP-B. It was also observed that, at early time points (day 1 and day 4), hBMSCs were more spread on TCP-S forming larger cell bodies than on TCP-B. Furthermore, primary cilia were more elongated on TCP-S at day 7 compared to TCP-B. After a 12-week intramuscular implantation in dogs, TCP-S induced bone formation while TCP-B did not. The results herein indicate that, in response to different surface structure of $\mathrm{CaP}$ ceramics, stem cells could undergo morphological changes and alter the structure of their primary cilia, resulting osteogenic differentiation and bone formation.
\end{abstract}

\section{Keywords:}

Calcium phosphate ceramic, Surface structure, Mesenchymal stromal cell, Cell morphology, Primary cilia, Osteogenic differentiation, Inductive bone formation. 


\subsection{Introduction}

Calcium phosphate ( $\mathrm{CaP}$ ) ceramics are widely used in orthopedic, dental and maxillofacial surgery as bone substitutes because of their chemical homology to native bone mineral, excellent biocompatibility and the ability to support osteogenesis on their surface (i.e. osteoconductivity) [1-3]. However, osteoinductivity of bone graft substitutes, i.e. the ability to positively induce osteogenic differentiation of stem cells to form bone, is thought essential for bone regeneration in critical-sized bone defects $[4,5]$. The most often used approach to make CaP ceramics osteoinductive is to combine them with growth factors (e.g. bone morphogenetic proteins, BMPs) [6]. However, the cost and safety of such approaches pose major concerns [7].

In the last decades, a subclass of $\mathrm{CaP}$ ceramics has been engineered to impart osteoinductivity without adding any osteogenic component, only by tailoring their physicochemical properties [8]. Among the physico-chemical properties important for osteoinductivity, micropores (i.e. pores smaller than $10 \mu \mathrm{m}$ ) have long been recognized as a crucial material factor. Given the same chemistry, macroporous hydroxyapatite (HA) ceramics with micropores on their surface gave rise to bone formation following either subcutaneous [9] or intramuscular [10] implantation, while those without micropores failed. Meanwhile, the osteoinductive potential of CaP ceramics was found to be correlated with microporosity: the higher the microporosity, the better inductive bone formation [11]. Most recently, when two tricalcium phosphate (TCP) ceramics having the same chemistry and microporosity were intramuscularly implanted using a canine ectopic model, the one presenting submicron-scaled pores $(0.65 \pm 0.25 \mu \mathrm{m}$, TCP-S) induced ectopic bone formation while the other with micro-scaled pores $(1.58 \pm 0.65 \mu \mathrm{m}, \mathrm{TCP}-\mathrm{B}) \operatorname{did}$ not $[12,13]$.

It is widely reported that the surface structure of a biomaterial can control fundamental cellular processes $[14,15]$. Adhesion of mesenchymal stromal cells (MSCs) was enhanced by a hybrid micron-/nano-structure of a grainy hardystonite-based surface rather than by a smoother HA-based substrate [16]. When MSCs were cultured on a polyurethane surface presenting domains of alternate grooves and ridges, pitch sizes of 1400 or $4000 \mathrm{~nm}$ led to greater cell elongation and alignment than $400 \mathrm{~nm}$ pitch surfaces or flat controls [17]. 


\section{Chapter 3}

Furthermore, it has been shown that the type of surface structure and its dimensional scale can influence on the differentiation fate of stem cells. For example, in the landmark study of McBeath et al. it was shown that MSCs commitment from adipogenic to osteogenic phenotypes could be switched by increasing the size of a fibronectin-coated surface domain from 1024 to $10000 \mu \mathrm{m}^{2}$ without the use of any osteogenic growth factors [18]. Not only could the size, the shape of the surface structure could be a cue to direct MSCs differentiation towards specific phenotypes as well [19]. These results led to the conclusion that specific geometrical characteristics of the underlying surface pattern can provide cells with instructive mechanochemical signals (i.e. from the shape and size of the underlying pattern element).

Despite several studies clearly showed the influence of surface properties on cell differentiation both in $2 \mathrm{D}$ and in $3 \mathrm{D}$ cell culture systems, the biological mechanism with which stem cells respond to surface structures and undergo osteogenic differentiation is still not entirely unraveled. Previous findings have indicated that cells could change their morphology according to the surface structure of the underneath substrates and differentiate into specific lineage $[20,21]$. Guvendiren and Burdick showed that the size and pattern of surface wrinkles could influence on MSCs morphology regulating their differentiation [22]. It was shown that osteogenic differentiation of hBMSCs was induced by high extracellular $\mathrm{Ca}^{2+}$ concentration through morphological changes with smaller nuclei and enlarged cytoskeleton [23]. Finally, cell morphology has been shown to correlate to specific activated pathways involved in the morphological regulation of stem cells, e.g. RhoA, which regulate the differentiation of MSCs without soluble factors towards specific phenotypes $[18]$.

In recent years, cells' primary cilia were reported acting as important sites for intracellular signaling able to detect external chemical and mechanical changes in the extracellular environment $[24,25]$, coordinating multiple signaling pathways such as receptor tyrosine kinase (RTK), Hedgehog (Hh), Wnt, Notch and mTOR. Thus, stem cell differentiation appears to be regulated by the primary cilium, whose presence and length are crucial factors [26-28]. For instance, human adipose-derived stem cells underwent osteogenic differentiation and exhibited longer cilia when cultured with the presence of dexamethasone [29], indicating 
that primary cilium is a chemical antenna regulating stem cell osteogenesis. Next to the chemical stimuli, cilia structure could be regulated by morphological change of the cells according to surface topography [30].

Therefore, we hypothesized that osteoinductive CaP ceramics could promote osteogenic differentiation of human bone marrow MSCs (hBMSCs) by altering cell morphology and subsequently influencing the development of primary cilia through surface topography. To demonstrate this hypothesis, we investigated the cell morphology of hBMSCs on TCP ceramics with two distinct surface micro-structures (namely TCP-B and TCP-S). We correlated cell morphology with the development of primary cilia, cell osteogenic differentiation and in vivo bone formation in a canine ectopic mode.

\subsection{Materials and methods}

\subsubsection{TCP-S and TCP-B ceramics}

TCP powders were prepared as previously described [13]. Briefly, a calcium hydroxide (Fluka) suspension and a phosphoric acid (Fluka) solution were mixed at a $\mathrm{Ca} / \mathrm{P}$ ratio of 1.50 . TCP-S and TCP-B powders were obtained by controlling the respective reaction rates. The powders were then mixed with diluted $\mathrm{H}_{2} \mathrm{O}_{2}(0.1 \%)$ (Merck) to obtain green bodies. The TCP-S and TCP-B ceramics were finally achieved by sintering the dry green bodies at $1050{ }^{\circ} \mathrm{C}$ (TCP-S) and $1100{ }^{\circ} \mathrm{C}$ (TCP-B) for 8 hours, respectively.

TCP-S and TCP-B discs ( $\$ 9 \times 1 \mathrm{~mm}$ ) were machined using a diamond-coated saw microtome (SP-1600, Leica, Germany) for in vitro evaluation. Ceramic cylinders ( $99 \times 12 \mathrm{~mm}$ ) with two transverse cuts of $1.1 \pm 0.1 \mathrm{~mm}$ were made as well for in vivo evaluation (Figure 1A). The obtained materials were then ultrasonically cleaned with acetone, $70 \%$ ethanol and demineralized water, and dried at $80^{\circ} \mathrm{C}$. All samples were steam sterilized at a pressure of 2-4 bar $\left(121^{\circ} \mathrm{C}\right)$ for $30 \mathrm{~min}$ and dried at $80^{\circ} \mathrm{C}$ afterwards.

Crystal chemistry of the TCP-S and TCP-B ceramics were determined with X-ray diffraction (XRD, Rigaku, Japan) and confirmed to be $\beta$-TCP. Microstructures (surface morphology, grain size and pore size) were observed with an environmental scanning electron microscope (ESEM; XL30, ESEMFEG, Philips, Eindhoven, The Netherlands) in the secondary electron 
mode. Porosity, pore distribution and total pore area were determined by mercury intrusion testing (Micromeritics, USA).

\subsubsection{Cell culture}

\subsubsection{Isolation and expansion of $h B M S C s$}

hBMSCs from donors were isolated from bone marrow aspirates with written informed consents as previously described [13]. In brief, aspirates from the donors were re-suspended using a $20 \mathrm{G}$ needle, plated at a density of $5 \times 10^{5}$ cells $/ \mathrm{cm}^{2}$ and cultured in proliferation medium (PM) containing basic medium (BM) and basic fibroblasts growth factor (bFGF, Instruchemie, the Netherlands, $1 \mathrm{ng} / \mathrm{mL}$ ). BM consisted of alpha-MEM (Life Techonologies) supplemented with $10 \%$ of fetal bovine serum (FBS, Life Technologies), $0.2 \mathrm{mM}$ ascorbic acid (ASAP, Life Technologies), 20 mM L-glutamine (Life Technologies), $100 \mathrm{U} / \mathrm{mL}$ penicillin (Life Technologies) and $100 \mu \mathrm{g} / \mathrm{mL}$ streptomycin (Life Technologies). Cells were expanded at $37^{\circ} \mathrm{C}$ in a humid atmosphere with $5 \% \mathrm{CO}_{2}$, medium was refreshed twice per week and cells were sub-cultured until 80-90\% confluence. Passage 2-3 hBMSCs were used.

\subsubsection{Cell culture on TCP ceramics}

To study the effect of surface structure on cellular behavior, hBMSCs were cultured on the TCP discs. All the discs were plated in non-treated 48-well plate and soaked in BM for at least four hours before cell seeding. Non-treated plates were used to minimize cell attachment on the bottom of the plates. To evaluate cell morphology and primary cilia development, cells were seeded onto the TCP discs at a density of 5,000 cells in $1 \mathrm{~mL}$ medium per sample. For cell proliferation, alkaline phosphatase (ALP) activity and gene expression, cells were seeded at a density of 25,000 cells per sample in $1 \mathrm{~mL}$. BM was mainly used to culture the cells on the TCP ceramic surface. When osteogenic medium (OM) was used, it contained $10^{-8} \mathrm{M}$ dexamethasone in addition to BM composition. Cells were cultured on ceramic discs at $37{ }^{\circ} \mathrm{C}$ in a humid atmosphere with $5 \% \mathrm{CO}_{2}$. The medium was refreshed twice per week.

\subsubsection{Cell morphology analysis}

For cell morphology assay, samples were harvested at day 1 and day 4 . The samples (donor 1 , $\mathrm{n}=3$ per condition) were fixated for 30 minutes in $4 \%$ paraformaldehyde, washed with PBS 
and permeabilized in $0.25 \%$ Triton-X 100 in PBS. Subsequently, F-actin was stained using phalloidin-AF488 (LifeTechnologies) and nuclei were stained using DAPI (Sigma Aldrich). After washing severely with PBS, montage images were captured using a BD Pathway system (BD Pathway 435, BD biosciences). The image analysis program Cell Profiler was used to quantitatively measure the morphological characteristics of cells cultured on TCP-B and TCPS scaffold materials. Depicted descriptors for cellular morphology were chosen based on relevance and statistically significant differences between TCP-S and TCP-B scaffolds. Such measures included the cell area and perimeter, the major and minor axis lengths and the form factor (ratio of minor over major axis lengths).

\subsubsection{Primary Cilia}

To evaluate the influence of surface structure on primary cilium development, cells (donor 4) cultured on TCP ceramics underwent serum starvation at day 7 for 24 hours, the samples ( $n=3$ per condition) were harvested and fixed with $3.7 \%$ paraformaldehyde at $37{ }^{\circ} \mathrm{C}$ for 8 minutes, permeabilized in $0.5 \%$ Tiriton and blocked with $5 \%$ goat serum thereafter. Immunostaining was started with anti-acetylated $\alpha$-tubulin primary antibody (clone 6-11 B-1, 1:2,000; Sigma-Aldrich) at $4{ }^{\circ} \mathrm{C}$ for overnight, then washed and incubated for 2 hours at $25^{\circ} \mathrm{C}$ with Alexa 488 Anti-mouse conjugate (Invitrogen). Finally, the samples were mounted with a DAPI counterstaining (invitrogen). Maximal projections of confocal z-stacks were created with a Leica SP2 confocal microscope. Cilia prevalence was assessed and the cilia length was measured using image J software subsequently [30].

To confirm the alteration of primary cilium structure in osteogenesis, cilia of hBMSCs, which were cultured on glass coverslips $(\varnothing 10 \mathrm{~mm})$ was evaluated in 48 -well plates with or without dexamethasone. hBMSCs were seeded at a cell density of 5,000 cells per sample cultured with a medium refreshment at day 4. At day 7, a 24-hour serum starvation was applied and the primary cilium development was immunochemically evaluated as described above.

\subsubsection{Cell proliferation and ALP assays}

For cell proliferation and ALP assays, samples (donor 1-3, $n=3$ per condition) were harvested at day 1 , day 4 , day 7 and day 14 respectively. The samples were gently rinsed three times with PBS, dried by aspirating PBS, and stored at $-20{ }^{\circ} \mathrm{C}$ until further use. $500 \mu \mathrm{L}$ of lysis buffer 


\section{Chapter 3}

(prepared according to manufacturer's instructions of CyQuant Cell Proliferation Assay kit instructions) was added onto each sample, followed by three cycles of freezing and thawing at $-20{ }^{\circ} \mathrm{C}$ and room temperature, respectively. Cell proliferation was analyzed with a DNA assay (CyQuant Cell Proliferation Assay kit, Sigma, the Netherlands), according to the manufacturer's instruction. Briefly, $100 \mu \mathrm{L}$ cell lysate and DNA standard were incubated with $100 \mu \mathrm{L}$ CyQuant GR dye at room temperature for 15 minutes and measured using a spectrophotometer (Victor, Perkin Elmer) at an excitation wavelength of $480 \mathrm{~nm}$ and emission wavelength of $520 \mathrm{~nm}$. ALP activity was measured using a CDP-star assay kit (Roche). Briefly, $40 \mu \mathrm{L}$ of CDP star substrate were incubated with $10 \mu \mathrm{L}$ cell lysate for 20 min after which luminescence was measured using a spectrophotometer (Victor, Perkin Elmer). ALP expression was normalized to DNA content.

In the cases of cell proliferation, ALP assays and gene expressions, the seeding density of cells was higher (25,000 cells), as compared to cell morphological and primary cilia assays (5000 cells). To confirm that the morphological difference of cells was still present in the high seeding density, cells on TCP discs were viewed at day 1 with methylene blue staining and SEM observations (donor 2). After fixing with 4\% paraformaldehyde and washing with PBS, the samples were stained with $1 \%$ methylene blue and viewed with a steromicroscope (LM; E600, Nikon SMZ-10A, Japan). Thereafter, the samples were dehydrated in sequential ethanol series and followed by critical point drying from liquid carbon dioxide using a Balzers CPD 030 Critical Point Dryer. The samples were gold sputter coated (Cressington) before being imaged by SEM.

To confirm the osteogenic differentiation of cells on TCP discs, ALP staining was conducted on the 7-day samples (donor 2), following the manufacturer's protocol of the CDP-star kit (Sigma-Aldrich). Samples were first washed three times with PBS, fixed with 4\% paraformaldehyde for 30 seconds, and then incubated for 30 minutes in staining solution containing Naphtol AS MX phosphate and Fast Blue. The ALP positive cells were stained blue. Finally, the samples were washed 3 times with deionized water and observed under the stereomicroscope (LM; E600, Nikon SMZ-10A, Japan). 


\subsubsection{Bone-related gene expression}

For gene expression, cells (donor 1 ) were cultured on TCP discs in both BM and OM. Samples ( $n=3$ per condition) were collected at day 4 , day 7 and day 14 . Bone-related gene expression was evaluated with quantitative real-time polymerase chain reaction (PCR) assay. RNA isolation was performed using Trizol reagent (Invitrogen) and Nucleospin RNA isolation kit (Macherey-Nagel Gmbh \& Co.) according to the manufacturer's instructions. Total RNA was measured using a NanoDrop spectrophotometer (Nanodrop technologies, USA). The RNA was used to synthesize complementary DNA (cDNA) with an iScript cDNA Synthesis kit (BioRad) according to the manufacturer's instructions. PCR analysis was performed with a Bio-Rad real-time PCR system (Bio-Rad, Hercules, CA, USA) on ALP, collagen type I (Col I), osteocalcin (OCN), and osteopontin (OPN), with beta-2 microglobulin (B2M) as the housekeeping gene used for normalization. Primer sequences for ALP, Col I, OCN, OPN, and B2M are listed in Table 2. The relative amounts of target genes normalized by B2M were calculated by $2^{-\Delta C \mathrm{~T}}$ method where $\Delta \mathrm{C}_{\mathrm{T}}=\mathrm{C}_{\mathrm{T}, \text { Target }}-\mathrm{C}_{\mathrm{T}, \mathrm{B} 2 \mathrm{M}}$.

Table 1. qPCR primer sequences

\begin{tabular}{lll}
\hline Gene & Forward primer & Reverse primer \\
\hline OCN & GGCAGCGAGGTAGTGAAGAG & GATGTGGTCAGCCAACTCGT \\
OPN & CCAAGTAAGTCCAACGAAAG & GGTGATGTCCTCGTCTGTA \\
ALP & ACAAGCACTCCCACTTCATC & TTCAGCTCGTACTGCATGTC \\
Col-I & AGGGCCAAGACGAAGACATC & AGATCACGTCATCGCACAACA \\
B2M & GACTTGTCTTTCAGCAAGGA & ACAAAGTCACATGGTTCACA \\
\hline
\end{tabular}

\subsubsection{In vivo bone formation}

TCP-B and TCP-S samples were implanted in an ectopic canine model for 12 weeks. Following the permission of the local animal care committee (Animal Center, Sichuan University, Chengdu, China), the TCP-S and TCP-B cylinders (Figure $1 \mathrm{~A}$ ) were implanted in the para-spinal muscles of 8 adult male dogs (mongrel, 10-15 kg). All surgeries were conducted under general anaesthesia by abdominal injection of sodium pentobarbital ( $30 \mathrm{mg} / \mathrm{kg}$ body weight) and sterile condition. Following the surgeries, buprenorphine (0.1 $\mathrm{mg}$ per animal) 
was intramuscularly given to the animals as pain relief for 2 days, while penicillin $(40 \mathrm{mg} / \mathrm{kg})$ was intramuscularly injected for 3 consecutive days to prevent infection. After operation, the animals were allowed for full weight bearing and received normal diet. After 12 weeks, the dogs were sacrificed by a celiac injection of excessive amount of pentobarbital sodium. Implants were harvested with surrounding tissues and fixed in $4 \%$ formaldehyde, and embedded in poly(methyl methacrylate) (PMMA) after a series of gradient ethanol dehydration. Non-decalcified sections were made by crossing the middle of the explants using a diamond saw (SP-1600, Leica, Germany) and stained with 1\% methylene blue (Sigma) and $0.3 \%$ basic fuchsine (Sigma) solutions. Histological observations were performed using light microscopy. The histological slides were scanned with a scanner (DIMAGE Scan Elite5400 II, model AF5400-2, KONICA MINOLTA) for histomorphometrical analysis and bone formation was quantified as coverage of the inner surface.

\subsubsection{Statistical analysis}

Quantitative results are shown as average \pm standard deviation. Multiple comparisons were performed with two way analysis of variance (ANOVA) followed by Bonferroni post-test comparisons. P-values lower than 0.05 were considered as statistically significant differences.

\subsection{Results}

\subsubsection{Characterization of the ceramics}

Figure $1 \mathrm{~A}$ showed the images of TCP samples for both in vitro and in vivo evaluation. Analysis of XRD (Figure 1B) clearly showed the two TCP ceramics had the same chemistry, pure $\beta$-TCP. TCP with different size of surface microstructure was prepared shown by SEM (Figure C, D), TCP-B contained larger grains and micropore size than those of TCP-S (Table 2). Porosity and total pore area as summarized in Table 2.

Table 2. Physical properties of TCP ceramics

\begin{tabular}{lll}
\hline Physical parameters & \multicolumn{1}{c}{ TCP-B } & \multicolumn{1}{c}{ TCP-S } \\
\hline Average grain size $(\mu \mathrm{m})$ & $3.40 \pm 0.82$ & $0.90 \pm 0.25$ \\
Average pore size $(\mu \mathrm{m})$ & $1.70 \pm 0.63$ & $0.67 \pm 0.30$ \\
Porosity $(\%)$ & 47 & 45 \\
Total pore area $\left(\mathrm{m}^{2} / \mathrm{g}\right)$ & 0.7 & 1.4 \\
\hline
\end{tabular}


Surface structure of calcium phosphate ceramics instructs inductive bone formation via influencing on morphology and primary cilia structure of stem cells

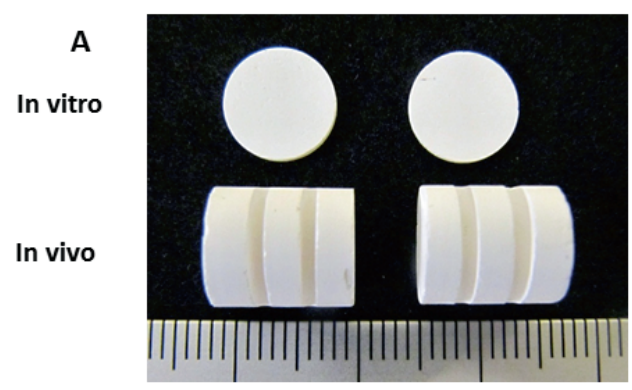

C

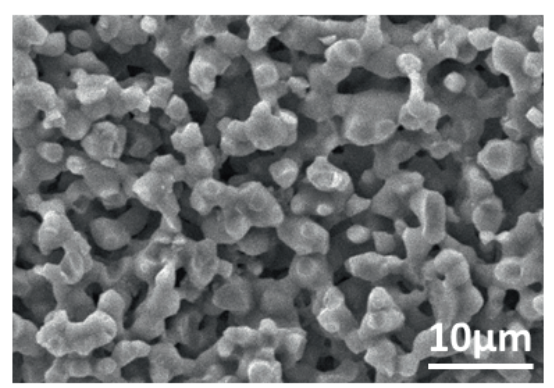

B Chemistry of TCP ceramics

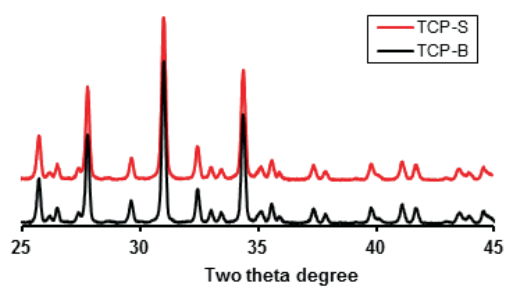

D

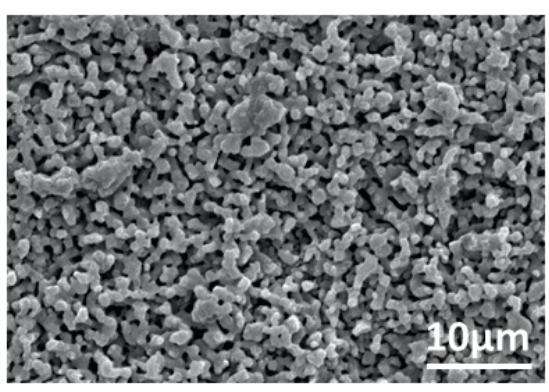

Figure 1. The images of samples for in vitro and in vivo evaluations (A); chemistry of TCP ceramics analyzed with XRD (B); SEM images of TCP-B (C) and TCP-S (D).

\subsubsection{Cell morphology of hBMSCs}

Fluorescent imaging showed that cells cultured on TCP-S were more spread and had larger area compared to those on TCP-B after 1 and 4 days (Figure 2A). The quantitative data indicated that the cells on TCP-S had significantly larger area, higher major and minor axis lengths, and longer perimeter, but similar form factor, compared to those on TCP-B (Figure 2B). The form factor values ranged between 0.3 and 0.4 for both TCP ceramics, suggesting that cells had elongated morphology on both materials. However, those on TCP-S were more spread than on TCP-B as shown by all the other morphological indicators. Evidently, the size of the surface microstructure influenced cell geometry after culturing hBMSCs on TCP discs for 1 and 4 days. 
A

Day 1

TCP-B

TCP-S

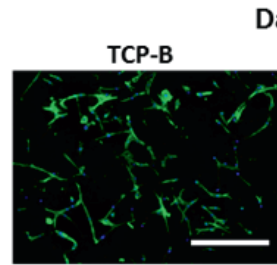

Day 4
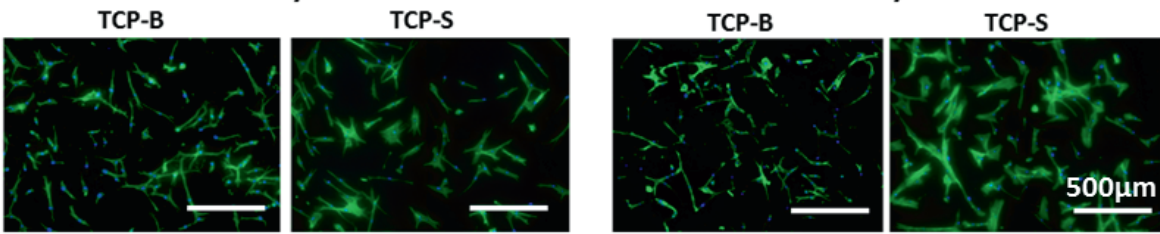

B
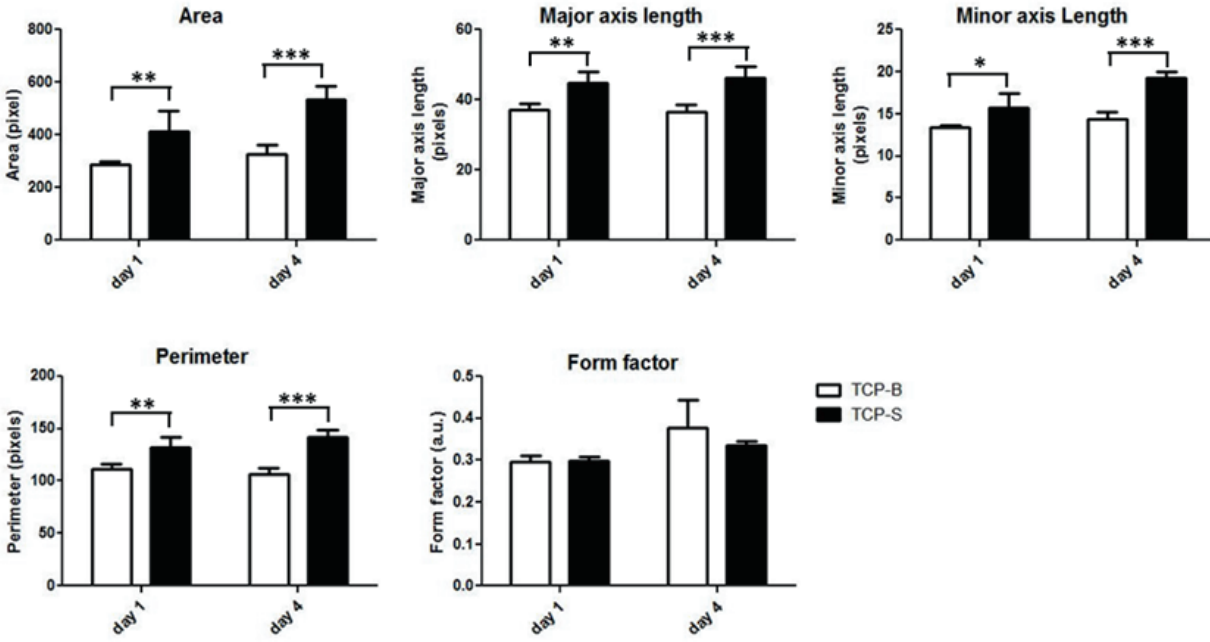

$\square$ TCP-B

Figure 2. Representative images of cell nucleus (DAPI) and actin skeleton (Phalloidin) of hBMSCS on TCP discs for 1 and 4 days (A); Analysis of morphological feature of actin skeleton in $h B M S C$ s cultured on TCP-S and TCP-B for 1 and 4 days (B).

\subsubsection{Primary Cilia}

Primary cilia could be viewed after immunostaining under confocal microscope in hBMSCs cultured on both glass coverslips and TCP ceramic surface (Figure 3, A to D). The incidence of primary cilia in hBMSCs was not much affected by dexamethasone on glass coverslips (Figure $3 A$, Figure $3 B$ and figure $3 E)$ with slight higher cilia prevalence in the case of $O M(p<0.05)$, while dexamethasone significantly increased the length of primary cilia (Figure 3F, $p<0.001$ ). Both incidence and length of primary cilia were affected by TCP ceramic surfaces (Figure 3, C to F). In particular, more and significantly longer primary cilia were detected on TCP-S than on TCP-B (Figure 3E, p<0.01; Figure 3F, $\mathrm{P}<0.001$ ). 
Surface structure of calcium phosphate ceramics instructs inductive bone formation via influencing on morphology and primary cilia structure of stem cells
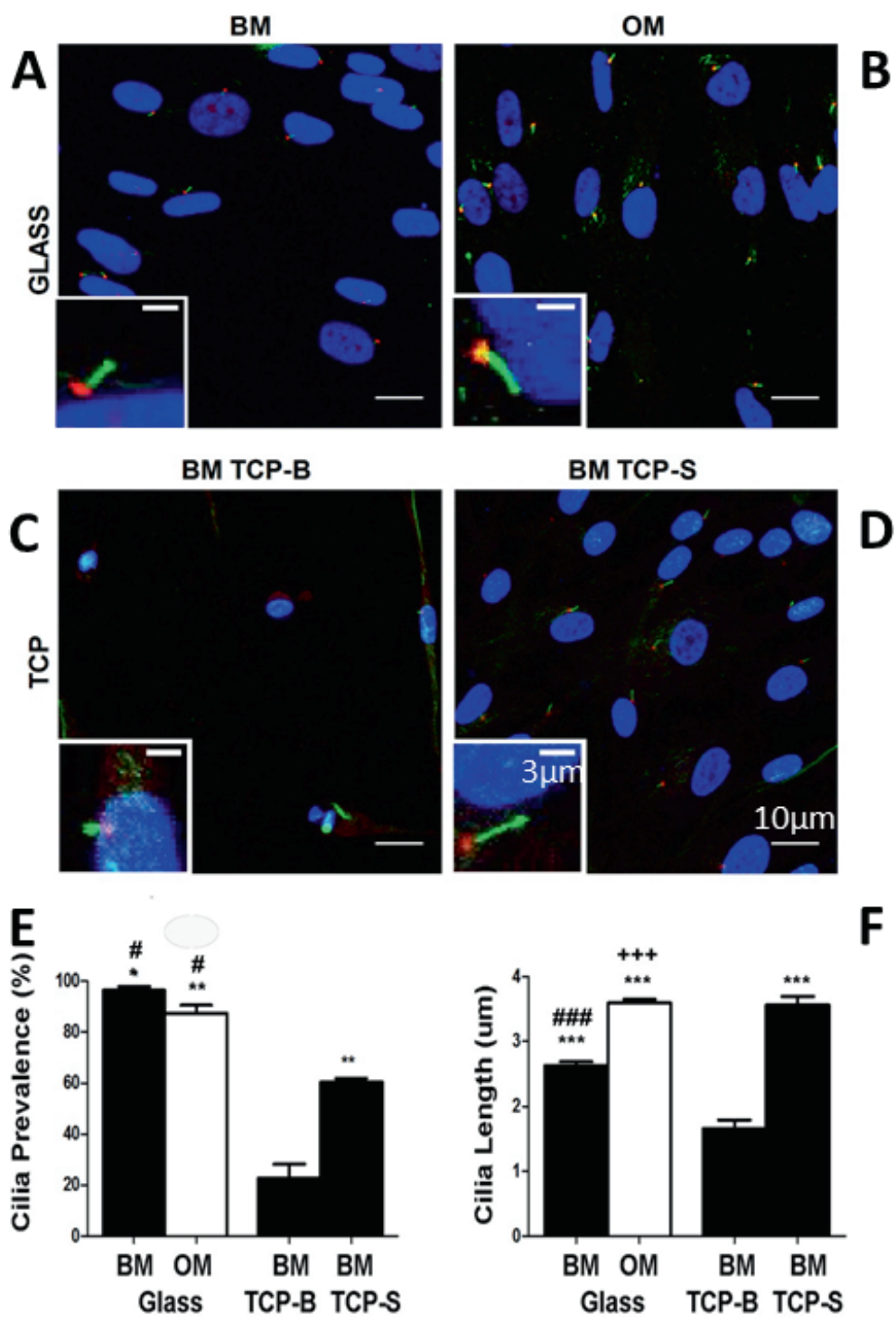

Figure 3. Primary cilium development in hBMSCs. A and B: primary cilia development of hBMSCs cultured on glass, showing longer primary cilia in OM medium (B) than in $B M(A) ; C$ and $D$ : primary cilia development of $h B M S C$ s cultured on TCP ceramics, showing longer primary cilia on TCP-S (D) than on TCP-B (C); E: primary cilia prevalence of hBMSCs cultured on glass as a function of Dex and on TCP ceramics as the function of surface structure in BM; F: primary length of hBMSCs cultured on glass as a function of Dex and on TCP ceramics as a function of surface structure in $B M\left({ }^{*} p<0.05, * * p<0.01\right.$ against TCP-B; \#P<0.05, \#\#\# $p<$ 0.001 against TCP-S; $+++p<0.001$ against BM). Scale bars are 10 micrometer for field images and 3 micrometer for single nuclei - cilia images 

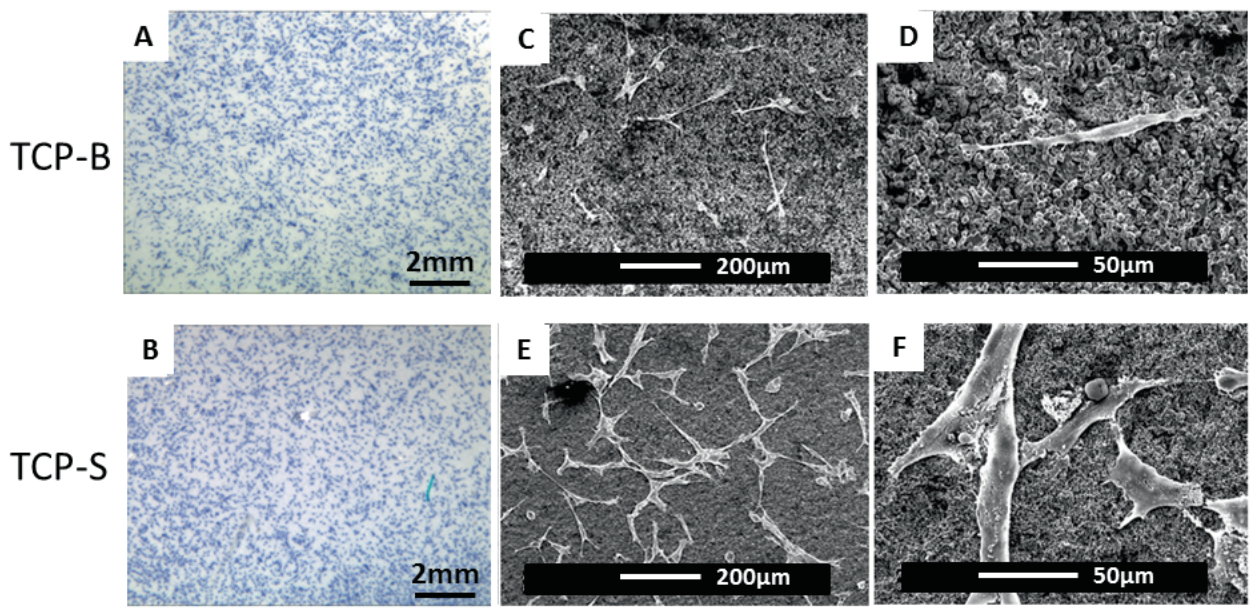

Figure 4. Methylene blue staining of hBMSCS cultured on TCP-B (A) and TCP-S (B) for 24 hours, showing cells attached homogeneously on both materials; SEM observation of hBMSCs on $T C P-B(C, D)$ and TCP-S (E, F) after 24 hours culture, showing the cells on TCP-S are more spread and larger than that on TCP-B.

Regardless of culture medium, more primary cilia could be detected in cells cultured on glass coverslips than on that on TCP-S and TCP-B (Figure 3E). Longer primary cilia were seen in cells cultured in both BM and OM on glass than on TCP-B in BM (Figure 3F). However, it is notable that primary cilia of cells cultured on TCP-S in BM were equivalent in length to those of cells cultured on glass in OM, and significantly longer than those of the cells cultured on glass in BM (Figure 3F).

\subsubsection{Distribution and attachment of hBMSCs on TCP ceramics}

To confirm the morphological changes preserved at the high cell density, distribution and attachment of hBMSCs on TCP ceramics were assessed with steromicroscope and SEM at day 1. Cells were homogeneously distributed on both TCP discs after 24 hours (Figure 4A and 4B). As shown in Figure 4 (C, D, E and F), the cell morphology on both TCP discs was elongated, but more spread and larger hBMSCs were on TCP-S than on TCP-B.

\subsubsection{Proliferation and osteogenic differentiation of hBMSCs}

To investigate whether the surface microstructure has an influence on the proliferation and osteogenic differentiation potential of hBMSCs, cells from three donors (Donor 1, 2 and 3) were cultured on TCP-B and TCP-S in BM. Cells grew from day 1 to day 14 on TCP-S for all the 
three donors (Figure 5A, DNA). Cells from donor 2 and 3 grew as well on TCP-B in time, while cells from Donor 1 proliferated less (Figure 5A). Surface-dependent cell proliferation was found from donor 1 and donor 2 with a significantly higher cell proliferation on TCP-S than on TCP-B (Figure 5A). No difference in cell proliferation was found between TCP-B and TCP-S in donor 3.
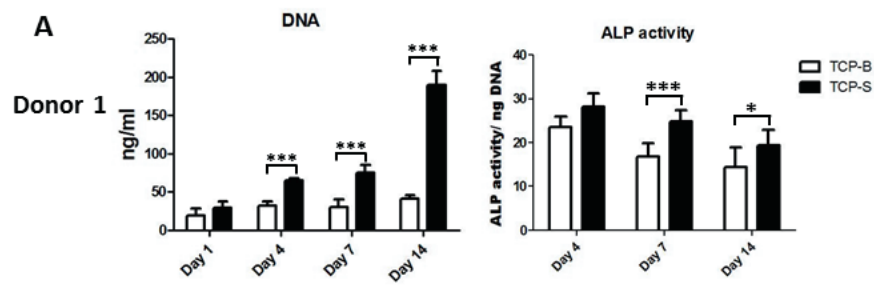

B
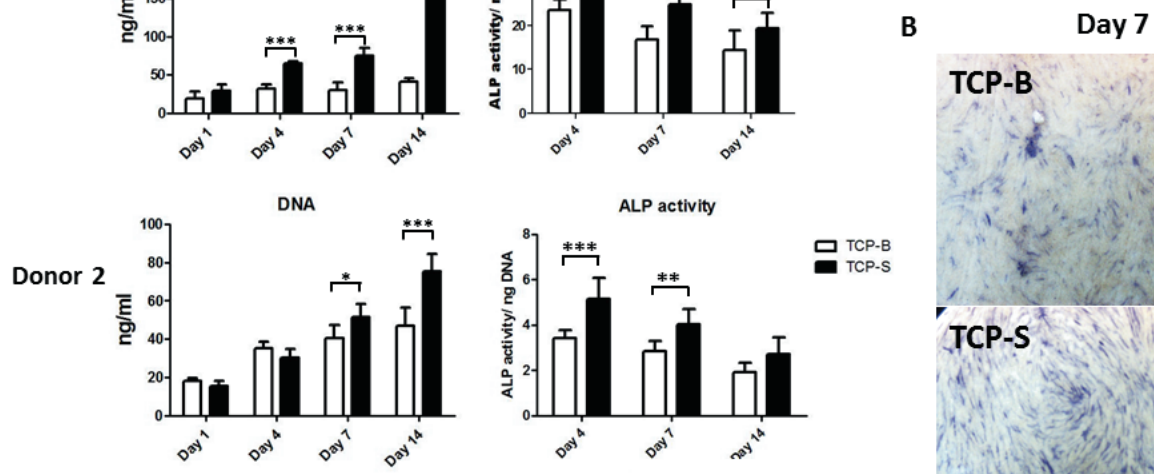

$\square$ TCP-B
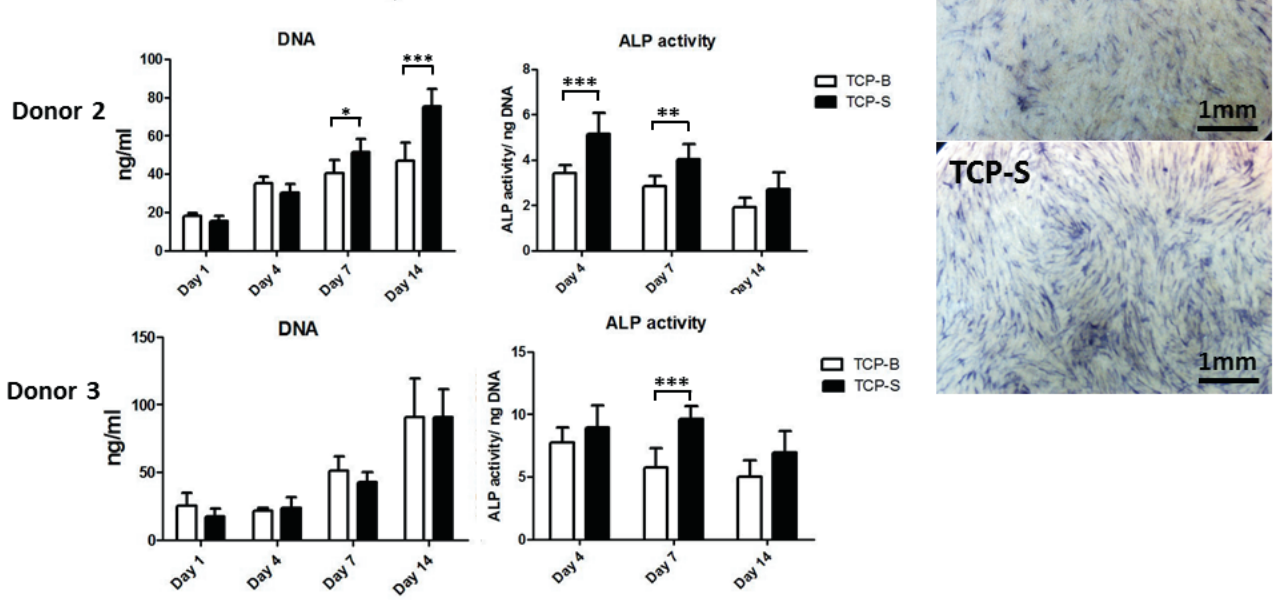

Figure 5. DNA quantification and ALP activity (normalized to DNA amount) of hBMSCs from 3 donors cultured on TCP discs overtime (A), showing significantly higher proliferation and osteogenic differentiation of $h B M S C S$ on TCP-S than that on TCP-B disCS; ALP staining of hBMSCs after culture on TCP discs 7 days (B), showing the blue was more intense on TCP-S.

To gain insight in the osteogenic differentiation of hBMSCs on TCP-B and TCP-S, ALP activity was determined. We observed that ALP production varied among the donors (Figure 5A, ALP activity). In particular, ALP activity slightly decreased over time from day 4 to 14 in cells from donors 1 and 2 on both TCP ceramics, while those from donor 3 did not change their ALP activity over time from day 4 to 14 . When TCP-S and TCP-B were compared, significantly higher ALP activity on TCP-S than on TCP-B could be observed (Donor 1: at day 7 and 14; Donor 2: at day 7 and 14; donor 3: at day 7, respectively). ALP immuno-staining of a set of 7day samples showed also a more pronounced ALP production on TCP-S (Figure 5B). 
Collagen type I

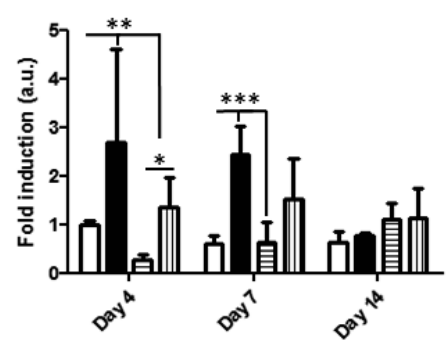

Osteocalcin

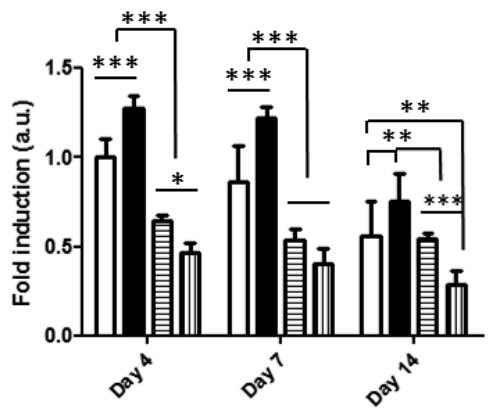

ALP

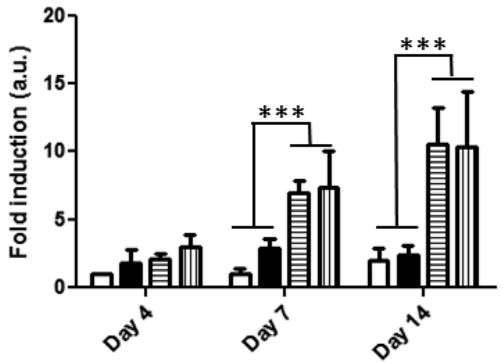

Osteopontin

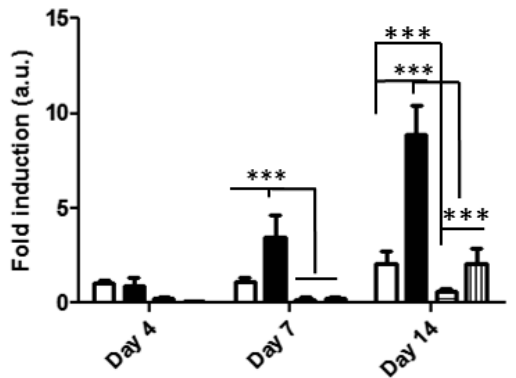

Figure 6. Osteogenic genes expression profile of $h B M S C S$ on TCP-S and TCP-B discs and in both BM and OM normalized to the B2M (housekeeping gene).

\subsubsection{Bone- related genes}

The possible influence of surface structure on osteogenic differentiation of hBMSCs was also assessed by gene expression analysis (qPCR) at days 4, 7 and 14. Figure 6 showed the relative gene expression of Col I, ALP, OCN and OPN in both BM and OM. In BM, a down regulation of Col I over time was found on TCP-S, while there was no significant change over time for hBMSCs on TCP-B. ALP gene expression was up-regulated from days 4 to 7, and slightly down-regulated from day 7 to 14 on TCP-S. There was no significant difference of ALP gene expression between day 4 and day 7, but slightly increased at day 14 on TCP-B. Down regulation of OCN from day 4 to day 14 was found on both TCP ceramics. The most pronounced enhancement of gene expression was seen for OPN on TCP-S, which was 4 times higher at day 7 and 10 times higher at day 14 than at day 4, but only slightly up regulated (less than 2 times) from day 4 to day 14 on TCP-B. For the comparison between the cells on TCP-S and TCP-B, Col I expression was significantly higher on TCP-S at days 4 and 7. Around a 3 times higher ALP gene expression was noted at day 7 on TCP-S than on TCP-B. 
Enhanced gene expression of OCN was also found on TCP-S at days 4 and 7, as compared to on TCP-B, although OCN gene expression was down-regulated with time. For OPN, importantly, it expressed 3 times higher at day 7 and 5 times higher at day 14 on TCP-S than on TCP-B.

When comparing the gene expression between $\mathrm{BM}$ and $\mathrm{OM}$, the presence of dexamethasone in OM down-regulated gene expression of col I, OCN and OPN, but upregulated gene expression of ALP. In OM, TCP-S enhanced col I gene expression at days 4 and 7, and OCN and OPN gene expressions at day 14. The enhancement of ALP gene expression by TCP-S in BM disappeared in OM.

\subsubsection{In vivo bone formation}

In total, 8 samples per TCP ceramic were harvested from 8 dogs for histological evaluation and histomorphometry. No bone formation was observed in any of the TCP-B samples (Figure 7A, C, E), while bone was formed in 7 out of 8 TCP-S explants (Figure 7B, D, F). Images with high magnification showed the presence of bone on the inner surface of TCP-S samples (Figure 7F) and only soft tissues were observed in TCP-B (Figure 7E). Quantitatively, $28 \pm 17 \%$ of the available inner surface of TCP-S explants was occupied by bone.

\subsection{Discussion}

Chemical design of materials and application of biological molecules are often used to achieve specific biological responses in tissue regeneration, while an increasing amount of evidences suggests that physical properties of biomaterials play a pivotal role for controlling biological functions as well [14]. In particular, it has been reported that the micro- and nanostructured surfaces of biomaterials can mediate cellular behavior including adhesion, morphology, proliferation and differentiation in vitro $[16,31]$. Here, we reported that surface structure not only affected cell fate in vitro, but also instructed specific tissue morphogenesis in vivo. TCP with submicron scaled surface features (TCP-S) instructed osteogenic differentiation of hBMSCs in vitro at both cellular level (Figure 5) and gene level (Figure 6). Following an ectopic implantation, such TCP-S ceramics gave rise to heterotopic bone formation in muscle; while TCP with micron scaled surface structure (TCP-B) did not (Figure 7). 
A
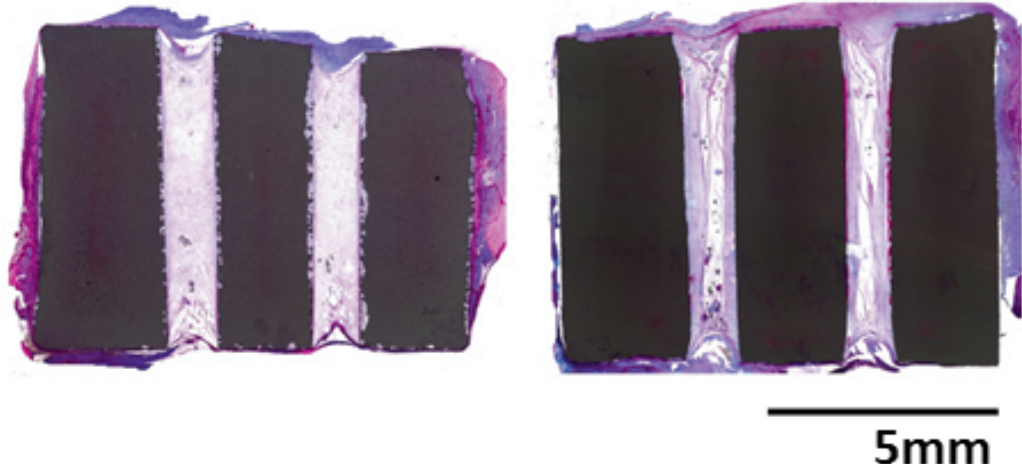

B

C
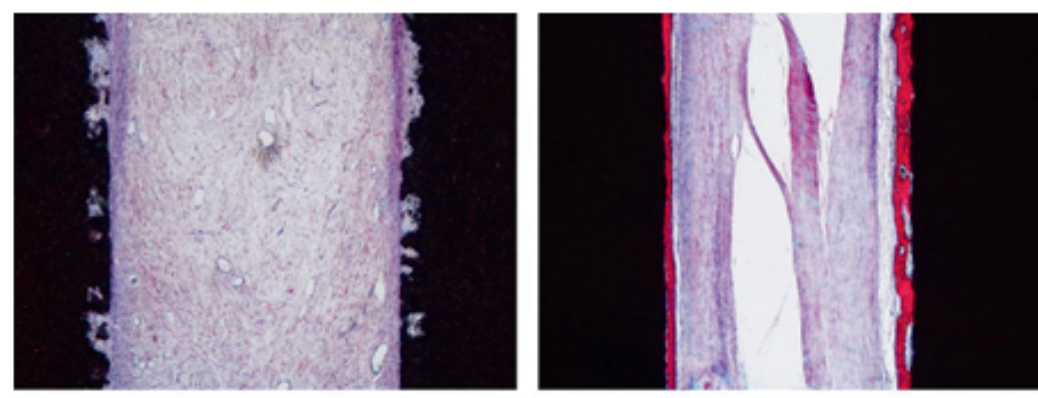

D

$500 \mu \mathrm{m}$
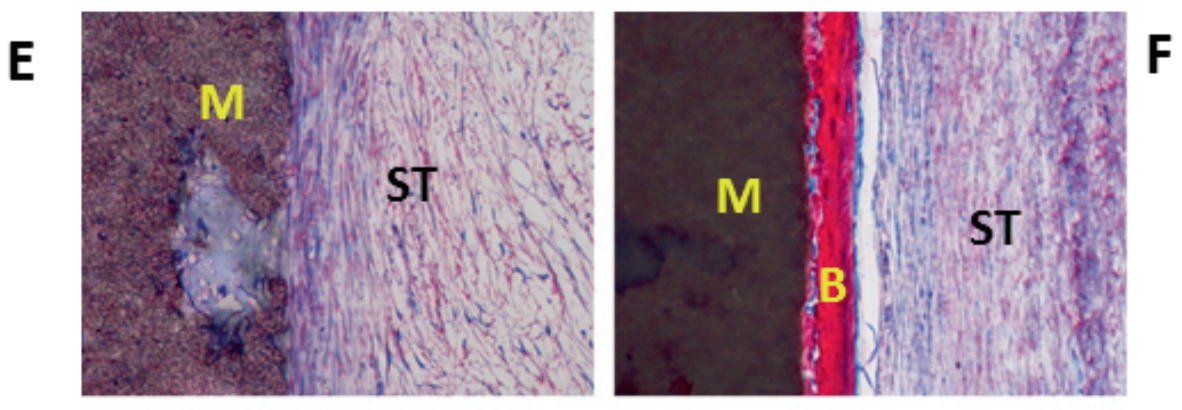

\section{$100 \mu \mathrm{m}$}

Figure 7. Histological overviews of $\operatorname{TCP}-B(A, C)$ and $\operatorname{TCP}-S(B, D)$ samples after a 12-week implantation in muscle of dog, showing bone formation in TCP-S and no bone in TCP-B; a high magnification image of TCP-B (E) explants, showing the infiltration of fibrous tissue but absence of bone in TCP-B; a high magnification image of TCP-S (F) explants, showing the presence of mineralized bone matrix (bright pink). (M: materials; B: bone; ST: soft tissue) 
The implantation of materials in vivo always follows the wound healing processes inherent of the innate immune reaction, followed by angiogenesis, tissue formation and remodeling. Many cell types (e.g. macrophages, myoblasts, MSCs and pericytes) involved in wound healing may be sensitive to surface structures and contribute to tissue morphogenesis. In the case of ectopic bone formation, an important step in the process of inducing new bone is thought to be the osteogenic differentiation of MSCs infiltrated into the implants [8]. A previous study has demonstrated that BMSCs could be one candidate playing roles in heterotopic bone formation guided by osteoinductive CaP ceramics [32]. hBMSCs were then used in the current study to further understand how stem cells respond to the surface structure of TCP ceramics during osteogenic differentiation.

The osteogenesis potential of stem cells was suggested to be dependent on the surface structure features of geometry and size, spatial organization and the dynamical changes of the surface properties [33]. In this study, TCP-S not only promoted ALP activity but also upregulated all bone-related gene expression tested, i.e. Col I, ALP, OCN and OPN (Figure 6A), which are pivotal factors in osteogenic differentiation, matrix deposition and mineralization $[13,34]$. Given the fact that no growth factors were used, these results indicate that the dimension of surface structure alone could instruct the osteogenic differentiation of hBMSCs. This result is in line with our previous data showing an osteogenic function of the scale of the surface structure in TCP ceramic granules [13].

The reaction of cells to the surface structure of the substrate on which they are cultured is one of the first steps occurring during the osteogenic differentiation process [35]. The probability of cells to make successful protrusions and contacts in a given direction varies with the extent and size of the surface feature [36]. In other words, cells adapt their morphology to the surface topographical characteristics of the substrates [37]. As a result, cells of the same phenotype can be different in shape, size, aspect ratio and height on materials with various surface topographies. It is known that cell differentiation is often associated with the morphological changes of the cells $[18,38]$. For instance, when hBMSCs were cultured on substrates presenting adhesive islands of various sizes, a shape change of hBMSCs according to the total accessible area was responsible for the switch in cell-lineage commitment to either adipogenic or osteogenic cells $[18,39]$. In particular, hBMSCs having 


\section{Chapter 3}

rounded morphology were differentiated into adipogenic cells, while spreading hBMSCs underwent osteogenic differentiation $[18,39]$. The correlation of cell morphology and osteogenic differentiation of hBMSCs was also seen in TCP ceramics in this study. Cells on both TCP-S and TCP-B were elongated, but those on TCP-S were more spread (Figure 2) and underwent osteogenic differentiation (Figure 5).

Through a change of internal cytoskeletal tension, a variation in cell morphology may change the expression of integrin, cadherins and cytoskeletal proteins $[18,40]$. As evidenced, a cell morphological change according to the surface structure was associated with the expression of primary cilia [41] and, more importantly, the primary cilia development was found correlated to the osteogenic differentiation of stem cells [42]. Human adipose-derived stem cells undergoing osteogenic differentiation have longer primary cilia [29]. In this study, we observed elongation of primary cilia in hBMSCs undergoing osteogenic differentiation. When cultured in OM, hBMSCs had longer primary cilia as compared to those in BM (Figure 3F). hBMSCs with enhanced osteogenic differentiation on TCP-S (Figure 5) had significantly longer primary cilia (Figure 3F) than TCP-B as well. How the elongation of primary cilia mediates osteogenic differentiation is not clear as yet, while literature data shows that primary cilia, via the associated proteins of polycystin-1 (PC1), polycystin-2 (PC2) and intraflagellar transport protein-88 (IFT88) [43], could affect a number of osteogenic signaling pathways, such as Wnt [44], runx2 [43], TGF- $\beta[30,43]$ and hedgehog [45].

The development of primary cilia in hBMSCs was affected by several experimental settings in this study. For example, dexamethasone did not affect much the incidence of primary cilia, but greatly influenced their length (Figure 3E and 3F). The surface topography significantly regulated both the incidence and length of primary cilia (Figure $3 \mathrm{E}$ and $3 \mathrm{~F}$ ). When taking the primary cilia length as the osteogenic marker of hBMSCs (Figure 3F), the influence of experimental settings on osteogenic differentiation could be seen. First, the surface structure at the submicron-scale could have the same osteogenic capacity of dexmethasone (TCP-S BM vs Glass OM, Figure 3F). Second, the role of surface structure dimension of TCP ceramics on osteogenic differentiation of hBMSCs could be seen, the submicron scaled surface is superior to the micron scale, when cells cultured in BM (Figure 3F). 
Dexamethasone was often used as osteogenic inducible molecules, because it is essential for the full differentiation of hBMSCs into mineral-producing osteoblasts [46, 47]. Not surprisingly, in this study, dexamethasone upregulated ALP expression of hBMSCs compared to cells cultured in BM for both TCP ceramics after 7 and 14 days. However, levels of Col I, OCN, and OPN were lower for cells cultured in OM compare to BM. Although dexmethasone in OM rapidly enhanced ALP expression, it decreased Col I, OCN and OPN mRNA levels. These data suggested that ALP expression could be uncoupled from Col I, OCN and OPN, as previously suggested that dexmethasone inhibited or postponed Col I and reduce OCN expression $[48,49]$.

The present study clearly showed a correlation between the scale of surface structure and in vivo bone morphogenesis as well as in vitro cellular behaviors of hBMSCs, including cell morphology, primary cilia development, osteogenic differentiation. The results indicate that, by adapting their morphology according to the surface structure of CaP ceramics, hBMSCs alter their primary cilia structure which in turn regulate osteogenic signaling pathways for bone formation. Understanding the complex interplay between these cellular behaviors and different surface architecture is a critical step toward a greater appreciation of how de novo bone formation occurs in synthetic bone grafting materials. Moreover, CaP ceramics with wider range of surface microstructure sizes should be tested to find if there is an optimal scale for promoting osteogenesis of stem cells. This would strengthen the evidence pointing to the suitable size of surface microstructure as a physical parameter for osteogenisis, which can easily be applied for the future design of CaP ceramics topography to induce bone formation.

\subsection{Conclusions}

A submicron scale surface structure of TCP ceramic scaffolds allowed a much more spread morphology of hBMSCs, elongated primary cilia, instructed osteogenic differentiation at a cellular and molecular level without any osteogenic factors. These scaffolds also induced in vivo bone formation. The correlation between the surface structure scale and the osteogenesis in both in vitro and in vivo implied a possible mechanism of surface structure driven bone formation in CaP ceramics. It is likely that, adapting surface structure, hBMSCs 
Chapter 3

change their morphology and develop primary cilia coupling to signal pathways of osteogenesis. 


\section{Surface structure of calcium phosphate ceramics instructs inductive bone formation via influencing on morphology and primary cilia structure of stem cells}

\section{References}

[1] Bohner M, Galea L, Doebelin N. Calcium phosphate bone graft substitutes: Failures and hopes. Journal of the European Ceramic Society. 2012;32:2663-71.

[2] Jarcho M. Calcium phosphate ceramics as hard tissue prosthetics. Clinical orthopaedics and related research. 1981;157:259-78.

[3] Samavedi S, Whittington AR, Goldstein AS. Calcium phosphate ceramics in bone tissue engineering: a review of properties and their influence on cell behavior. Acta Biomaterialia. 2013;9:8037-45.

[4] Habibovic P, Van der Valk C, Van Blitterswijk C, De Groot K, Meijer G. Influence of octacalcium phosphate coating on osteoinductive properties of biomaterials. Journal of materials science Materials in medicine. 2004;15:373-80.

[5] Yuan H, Van Blitterswijk C, De Groot K, De Bruijn J. A comparison of bone formation in biphasic calcium phosphate (BCP) and hydroxyapatite (HA) implanted in muscle and bone of dogs at different time periods. Journal of Biomedical Materials Research Part A. 2006;78:139-47.

[6] Ludwig SC, Kowalski JM, Boden SD. Osteoinductive bone graft substitutes. European Spine Journal. 2000;9:S119-S25.

[7] Williams BJ, Smith JS, Fu K-MG, Hamilton DK, Polly Jr DW, Ames CP, et al. Does bone morphogenetic protein increasethe incidence of perioperative complicationsin spinal fusion?: A comparison of 55,862 cases of spinal fusion with and without bone morphogenetic protein. Spine. 2011;36:1685-91.

[8] Barradas AM, Yuan H, van Blitterswijk CA, Habibovic P. Osteoinductive biomaterials: current knowledge of properties, experimental models and biological mechanisms. European cells \& materials. 2011;21:407-29;

[9] Yamasaki H, Sakai H. Osteogenic response to porous hydroxyapatite ceramics under the skin of dogs. Biomaterials. 1992;13:308-12.

[10] Yuan H, Kurashina K, de Bruijn JD, Li Y, De Groot K, Zhang X. A preliminary study on osteoinduction of two kinds of calcium phosphate ceramics. Biomaterials. 1999;20:1799-806.

[11] Hing KA, Revell PA, Smith N, Buckland T. Effect of silicon level on rate, quality and progression of bone healing within silicate-substituted porous hydroxyapatite scaffolds. Biomaterials. 2006;27:5014-26.

[12] Davison N, Luo X, Schoenmaker T, Everts V, Yuan H, Barrère-de Groot F, et al. Submicron-scale surface architecture of tricalcium phosphate directs osteogenesis in vitro and in vivo. European cells \& materials. 2013;27:281-97.

[13] Zhang J, Luo X, Barbieri D, Barradas A, de Bruijn JD, van Blitterswijk CA, et al. The size of surface microstructures as an osteogenic factor in calcium phosphate ceramics. Acta biomaterialia. 2014;10:3254-63.

[14] Mitragotri S, Lahann J. Physical approaches to biomaterial design. Nat Mater. 2009;8:15-23.

[15] Kim KS, Zhao Y, Jang H, Lee SY, Kim JM, Kim KS, et al. Large-scale pattern growth of graphene films for stretchable transparent electrodes. Nature. 2009;457:706-10.

[16] Zhang W, Wang G, Liu Y, Zhao X, Zou D, Zhu C, et al. The synergistic effect of hierarchical micro/nanotopography and bioactive ions for enhanced osseointegration. Biomaterials. 2013;34:3184-95.

[17] Watari S, Hayashi K, Wood JA, Russell P, Nealey PF, Murphy CJ, et al. Modulation of osteogenic differentiation in hMSCs cells by submicron topographically-patterned ridges and grooves. Biomaterials. 2012;33:128-36.

[18] McBeath R, Pirone DM, Nelson CM, Bhadriraju K, Chen CS. Cell shape, cytoskeletal tension, and RhoA regulate stem cell lineage commitment. Developmental cell. 2004;6:483-95.

[19] Kilian KA, Bugarija B, Lahn BT, Mrksich M. Geometric cues for directing the differentiation of mesenchymal stem cells. Proceedings of the National Academy of Sciences of the United States of America. 2010;107:4872-7.

[20] Carvalho R, Schaffer J, Gerstenfeld L. Osteoblasts induce osteopontin expression in response to attachment on fibronectin: demonstration of a common role for integrin receptors in the signal transduction processes of cell attachment and mechanical stimulation. Journal of cellular biochemistry. 1998;70:376-90.

[21] Spiegelman BM, Ginty CA. Fibronectin modulation of cell shape and lipogenic gene expression in 3T3adipocytes. Cell. 1983;35:657-66.

[22] Guvendiren M, Burdick JA. The control of stem cell morphology and differentiation by hydrogel surface wrinkles. Biomaterials. 2010;31:6511-8. 


\section{Chapter 3}

[23] Barradas AM, Fernandes HA, Groen N, Chai YC, Schrooten J, van de Peppel J, et al. A calcium-induced signaling cascade leading to osteogenic differentiation of human bone marrow-derived mesenchymal stromal cells. Biomaterials. 2012;33:3205-15.

[24] Eggenschwiler JT, Anderson KV. Cilia and developmental signaling. Annual review of cell and developmental biology. 2007;23:345.

[25] Abou Alaiwi WA, Lo ST, Nauli SM. Primary cilia: highly sophisticated biological sensors. Sensors. 2009;9:7003-20.

[26] Zhu D, Shi S, Wang H, Liao K. Growth arrest induces primary-cilium formation and sensitizes IGF-1-receptor signaling during differentiation induction of 3T3-L1 preadipocytes. Journal of cell science. 2009;122:2760-8.

[27] Nathwani BB, Yang TT, Liao J-C. Towards a subdiffraction view of motor-mediated transport in primary cilia. Cellular and Molecular Bioengineering. 2013;6:82-97.

[28] Yang S, Wang C. The intraflagellar transport protein IFT80 is required for cilia formation and osteogenesis. Bone. 2012;51:407-17.

[29] Bodle JC, Rubenstein CD, Phillips ME, Bernacki SH, Qi J, Banes AJ, et al. Primary cilia: the chemical antenna regulating human adipose-derived stem cell osteogenesis. PLoS One. 2013;8:e62554.

[30] McMurray R, Wann A, Thompson C, Connelly J, Knight M. Surface topography regulates wnt signaling through control of primary cilia structure in mesenchymal stem cells. Scientific reports. 2013;3.

[31] Meyer U, Buchter A, Wiesmann H, Joos U, Jones D. Basic reactions of osteoblasts on structured material surfaces. European cells \& materials. 2005;9:39-49.

[32] Song G, Habibovic P, Bao C, Hu J, van Blitterswijk CA, Yuan H, et al. The homing of bone marrow MSCs to non-osseous sites for ectopic bone formation induced by osteoinductive calcium phosphate. Biomaterials. 2013.

[33] Kiang JD, Wen JH, del Álamo JC, Engler AJ. Dynamic and reversible surface topography influences cell morphology. Journal of Biomedical Materials Research Part A. 2013;101:2313-21.

[34] Sun H, Wu C, Dai K, Chang J, Tang T. Proliferation and osteoblastic differentiation of human bone marrowderived stromal cells on akermanite-bioactive ceramics. Biomaterials. 2006;27:5651-7.

[35] Harrison RG. The reaction of embryonic cells to solid structures. Journal of Experimental Zoology. 1914;17:521-44.

[36] Clark P, Connolly P, Curtis A, Dow J, Wilkinson C. Topographical control of cell behaviour. I. Simple step cues. Development. 1987;99:439-48.

[37] Carman ML, Estes TG, Feinberg AW, Schumacher JF, Wilkerson W, Wilson LH, et al. Engineered antifouling microtopographies-correlating wettability with cell attachment. Biofouling. 2006;22:11-21.

[38] Dalby M, Riehle M, Johnstone H, Affrossman S, Curtis A. In vitro reaction of endothelial cells to polymer demixed nanotopography. Biomaterials. 2002;23:2945-54.

[39] Gao L, McBeath R, Chen CS. Stem Cell Shape Regulates a Chondrogenic Versus Myogenic Fate Through Rac1 and N - Cadherin. Stem Cells. 2010;28:564-72.

[40] Chou L, Firth JD, Uitto V-J, Brunette DM. Substratum surface topography alters cell shape and regulates fibronectin mRNA level, mRNA stability, secretion and assembly in human fibroblasts. Journal of cell science. 1995;108:1563-73.

[41] Shah JV. Cells in tight spaces: the role of cell shape in cell function. The Journal of cell biology. 2010;191:233-6.

[42] Tummala P, Arnsdorf EJ, Jacobs CR. The role of primary cilia in mesenchymal stem cell differentiation: a pivotal switch in guiding lineage commitment. Cellular and Molecular Bioengineering. 2010;3:207-12.

[43] Horani A, Brody SL, Ferkol TW, Shoseyov D, Wasserman MG, Ta-shma A, et al. CCDC65 mutation causes primary ciliary dyskinesia with normal ultrastructure and hyperkinetic cilia. PLoS One. 2013;8:e72299.

[44] Lienkamp S, Ganner A, Walz G. Inversin, Wnt signaling and primary cilia. Differentiation. 2012;83:S49-S55.

[45] Goetz SC, Anderson KV. The primary cilium: a signalling centre during vertebrate development. Nature Reviews Genetics. 2010;11:331-44.

[46] Cheng S-L, Yang JW, Rifas L, Zhang S-F, Avioli LV. Differentiation of human bone marrow osteogenic stromal cells in vitro: induction of the osteoblast phenotype by dexamethasone. Endocrinology. 1994;134:277-86.

[47] Jaiswal N, Haynesworth SE, Caplan AI, Bruder SP. Osteogenic differentiation of purified, culture - expanded human mesenchymal stem cells in vitro. Journal of cellular biochemistry. 1997;64:295-312. 
Surface structure of calcium phosphate ceramics instructs inductive bone formation via influencing on morphology and primary cilia structure of stem cells

[48] Fromigué O, Marie PJ, Lomri A. Differential effects of transforming growth factor $\beta 2$, dexamethasone and 1 , 25-dihydroxyvitamin D on human bone marrow stromal cells. Cytokine. 1997;9:613-23.

[49] Beresford J, Bennett J, Devlin C, Leboy P, Owen M. Evidence for an inverse relationship between the differentiation of adipocytic and osteogenic cells in rat marrow stromal cell cultures. Journal of cell science. 1992;102:341-51. 



\section{Chapter 4}

\section{Calcium phosphate ceramics initiate osteogenic response through topographical cues}

Jingwei Zhang ${ }^{\mathrm{a}, \mathrm{b}}$, Guilhermina A. M. Carriche ${ }^{\mathrm{a}}$, Filipa V. De Sousa ${ }^{\mathrm{a}}$, Xiaoman Luo ${ }^{\mathrm{c}, \mathrm{d}}$, Davide Barbieri $^{c}$, Febriyani Damanik ${ }^{a}$, Jeroen J.J.P. van den Beucken ${ }^{e}$, Joost D. de Bruijn ${ }^{c, d, f}$, Clemens A. van Blitterswijk ${ }^{\mathrm{a}, \mathrm{g}}$, Lorenzo Moroni ${ }^{\mathrm{a}, \mathrm{g}}$, Huipin Yuan ${ }^{\mathrm{a}, \mathrm{c}, \mathrm{g}, \mathrm{h}, *}$

a Department of Tissue Regeneration, MIRA Institute for Biomedical Technology and Technical Medicine, University of Twente, P.O. Box 217, 7500AE Enschede, The Netherlands

${ }^{b}$ Key Laboratory of Advanced Technologies of Materials, Ministry of Education, School of Materials Science and Engineering, Southwest Jiaotong University, Chengdu, P.R. China

${ }^{c}$ Xpand Biotechnology BV, Bilthoven, The Netherlands

${ }^{d}$ Department of Biomaterials Science and Technology, MIRA Institute for Biomedical Technology and Technical Medicine, University of Twente, P.O. Box 217, 7500AE Enschede, The Netherlands

${ }^{e}$ Department of Biomaterials (309), Radboudumc, P.O. Box 9101, 6500HB Nijmegen, The Netherlands

${ }^{f}$ School of Engineering and Materials Science (SEMS), Queen Mary University of London, E1 4NS London, United Kingdom

g Complex Tissue Regeneration department, MERLN Institute for Technology Inspired Regenerative Medicine, Maastricht University, Universiteitsingel 40, 6229 ER, The Netherlands

${ }^{h}$ Colleage of Physical Science and Technology, Sichuan University, Chengdu, China 


\begin{abstract}
At certain scale, the surface structure of calcium phosphate (CaP) ceramics instructs the osteogenic differentiation of stem cells and makes such CaP ceramics osteoinductive. For instance, tri-calcium phosphate (TCP) ceramics with submicron scale surface structures possess osteoinductive capacity both in vitro and in vivo, while those with micron scale surface structures have not. However, it is unknown if the surface structure instructs osteogenesis via chemical cues of protein adsorption, ion exchange and surface reprecipitation or surface topographic cue. To answer this question, TCP ceramic discs with a submicron scale surface structure and smooth glass-slides were coated with carbon or gold and cultured in vitro with human bone marrow stromal cells (hBMSCs) in basic medium without any osteogenic-inducing factors. Irrespective of thin coatings on submicron scale surface structured TCP ceramics, all the TCP-based materials had the same surface topography and provoked the same chemical changes in the culture medium. Cell morphology, proliferation, and osteogenic differentiation were comparable for all TCP-based materials irrespective of coatings. These results hence show that the surface chemical changes of carbon or gold coatings do not affect the osteogenic function of the submicron scale surface structure. The possible influence of carbon and gold coating on hBMSCs was further excluded when cells were cultured also on glass slides irrespective of coatings. Cells performed similar on all materials with respect to their morphology, proliferation and osteogenic differentiation. However, as compared to TCP-based materials, the osteogenic differentiation of hBMSCs on glass-based materials was inferior, suggesting that the surface topography directly instructs the osteogenic differentiation of mesenchymal stromal cells and, thus, is the key factor to initiate inductive bone formation for osteoinductive CaP ceramics.
\end{abstract}

\title{
Keywords:
}

Calcium phosphate ceramic, Surface structure, Topographical cue, Mesenchymal stromal cells, Osteogenesis, Osteoinduction. 


\subsection{Introduction}

It is becoming increasingly evident that calcium phosphate ( $\mathrm{CaP}$ ) ceramics are of great importance for application as bone graft substitutes because their chemical composition resembles that of bone mineral and excellent biological properties [1-5]. These biological properties allow CaP ceramics to guide and support bone formation from the host bone along their surface (i.e. osteoconduction), which ensures bone regeneration in non-criticalsized bone defects. Next to osteoconductive property, a specific group of CaP ceramics could be osteoinductive and trigger the differentiation of stem cells into osteogenic cells able to form bone in ectopic sites. When both osteoconductive and osteoinductive properties are present, CaP ceramics could hypothetically repair critical-sized bone defects [6] and achieve equal performance compared to autologous bone grafts or synthetic grafts endowed with recombinant human bone morphogenetic protein 2 (rhBMP-2) in repairing critical-sized bone defects [4].

CaP ceramics are generally osteoconductive because their chemistry allows the formation of a biological apatite layer onto their surface [1-3, 5], osteoinduction of CaP ceramics is material-dependent $[4,6,7]$. Physicochemical properties, such as geometry $[8,9]$, implant size [10], presence of micropores [11, 12], microporosity [13] and the dimension of surface structures $[14,15]$, have all shown their influence on the osteoinductive capacity of CaP ceramics. Among the physicochemical properties, surface microstructures with dimensions smaller than $10 \mu \mathrm{m}$ have shown to be crucial for osteoinduction of CaP ceramics. It has been reported that hydroxyapatite $(\mathrm{HA})$ ceramics with micropores are osteoinductive after subcutaneous [11] and intramuscular [12] implantation in dogs, while no bone formed in those HA ceramics lacking microstructures. Osteoinductive potential increased with microporosity [13]. Next to the presence of microstructure and microporosity, the dimension of micropores has been proven to be crucial. For instance, having the same microprorosity, tri-calcium phosphate (TCP) ceramics with a submicron scale surface structure have demonstrated to possess osteoinductive capacity in vitro and in vivo, while the same was not observed for those with micron scale surface [14-18].

The exact biological mechanism of material-induced bone formation still remains unclear. It is apparent that surface microstructures increase the total surface area and in that way may 
be able to locally increase the concentration of growth factors and ions within the microenvironments at the surface $[4,12,19,20]$. For instance, bone morphogenetic proteins (BMPs; e.g. BMP-2, BMP-6, BMP-7 and BMP-9) are induce bone formation in vivo [4, 21] and promote osteogenic differentiation of stem cells in vitro [21, 22]. Dexamethasone is a frequently used biological factor to trigger osteogenic differentiation of mesenchymal stromal cells (MSCs) in vitro [23]. Calcium, which can release from resorbable CaP ceramics, enhances cellular proliferation and induced osteogenic differentiation of pre-osteoblasts [24], osteoblasts [25], and human periosteal derived stem cells (hPDCs) [26]. Furthermore, Ca enhanced cell proliferation of the pre-osteoblast cell line MC3T3-E1 in a dose-dependent manner [27]. Upon treatment with $\mathrm{Ca}^{2+}$ ions, the morphology of hPDCs changes from fibroblastic to cuboidal, which is a hallmark of osteoblasts, along with an increase in gene expression of the osteogenic genes osteopontin (OPN), osteocalcin (OCN), and BMP-2 [26]. Next to hPDCs, human bone marrow MSCs (hBMSCs) have shown enhanced osteogenic gene expression of OP, OC and BMP-2 when cultured in medium with high concentration of $\mathrm{Ca}^{2+}$ (7.8 $\mathrm{mM}$ ) [28]. In addition, P released from the resorbed CaP ceramics can play a role in osteogenesis. For instance, high concentrations of $\mathrm{P}$ ions induced a phenotypic transformation of vascular smooth muscle cells into osteogenic-like cells that exert vesiclemediated calcification [29]. It is likely that CaP ceramics trigger osteoinduction via the chemical cues of protein adsorption and ion release, while it has been shown that at certain scale, surface structure enhanced osteogenic differentiation of MSCs [15, 30, 31] and gave rise to inductive bone formation $[15,18]$. It could well be that topographic cues created by the surface structure play important roles in inductive bone formation in CaP ceramics.

Hence, the goal in this paper is to distinguish the impact of chemical and topographic cues found in osteoinduction of CaP ceramics and to determine the role of CaP ceramic's surface topography in driving osteoinduction. To accomplish this, we altered the sub-micron structured TCP ceramic disc's and smooth glass slide's surface chemistry via thin carbon and gold coatings and evaluated the responses of hBMSCs to the surface modification in vitro. 


\subsection{Materials and methods}

\subsubsection{TCP, TCP-C and TCP-Au discs}

\subsubsection{Preparation of TCP, TCP-C and TCP-Au submicron-structured materials}

Calcium hydroxide (Fluka) and phosphoric acid (Fluka) solutions were mixed at a $\mathrm{Ca} / \mathrm{P}$ ratio of 1.50 to obtain a slurry that was subsequently aged, filtered, dried and grinded to get a powder. The powder was then mixed with diluted $\mathrm{H}_{2} \mathrm{O}_{2}(0.1 \%$, Fluka), vacuum filtered, dried at room temperature and sintered at $1050^{\circ} \mathrm{C}$ for 8 hours to get TCP ceramic green bodies. Ceramic discs $(\varnothing 9 \times 1 \mathrm{~mm})$ were then fabricated via machining the bodies with lathe and diamond saw (SP-1600, Leica, Germany). Before further use, the discs were ultrasonically cleaned with acetone, $70 \%$ ethanol and demineralized water, and then dried at $80^{\circ} \mathrm{C}$. To obtain carbon coated TCP (i.e. TCP-C), the discs were subjected to physical vapour deposition treatment using carbon as a substrate (Edwards 306) at a voltage/current of $36 \mathrm{~V} / 60 \mathrm{~mA}$ with a vacuum of $10 \times 10^{-5} \mathrm{mbar}$ for $4-5$ seconds. Gold coated TCP (i.e. TCP-Au) was prepared by treating discs with two cycles of 90 seconds using a sputter coater (Cressington Sputter Coater 108 Auto) at a current of $30 \mathrm{~mA}$. All samples were steam sterilized at a pressure of $1-1.1$ bar at $120{ }^{\circ} \mathrm{C}$ for $30 \mathrm{~min}$ and then dried at $80^{\circ} \mathrm{C}$ afterwards.

\subsubsection{Physico-chemical properties of TCP, TCP-C and TCP-Au surface}

The chemical composition of TCP, TCP-C and TCP-Au surfaces was determined with Energydispersive X-ray spectroscopy (EDAX, AMETEK Materials Analysis Division). The surface structure of each material was observed with an environmental scanning electron microscope (ESEM; XL30, ESEM FEG, Philips) in the secondary electron mode. The surface roughness was analyzed with an atomic force microscopy (AFM; PicoScan Controller 2500 Quadrexed Multimode; Molecular Imaging). A super sharp TESP cantilever (42N/m, $320 \mathrm{kHz}$, 2-5nm ROC, no coatings; Bruker AFM Probes) was used in tapping mode. AFM images were taken at a scan rate of $1 \mathrm{~Hz}$ on a $100 \mu \mathrm{m}^{2}$ of three different surface points for each sample $(n=3)$ using Nanoscope $\left(612 \mathrm{r}^{\circledR}{ }^{\circledR}\right.$, Digital Instrument Veeco). Obtained images were then analyzed with the Scanning Probe Image Processor (SPIPTM, version 4.2.2.0) to obtain roughness measurements ( $R a, R q$, and $R m a x)$ and high quality 3D images $(n=3)$. 


\subsubsection{Protein adsorption by TCP, TCP-C and TCP-Au materials}

To quantify change of protein concentration in culture medium, discs of all three TCP-based materials were immersed (in triplicates; $n=3$ ) in $1 \mathrm{~mL}$ basic culture medium (BM), consisting of Minimal Essential Medium-alpha ( $\alpha$-MEM, Gibco) supplemented with $10 \%$ fetal bovine serum (FBS, Lonza), $100 \mathrm{U} / \mathrm{mL}$ penicillin and $100 \mu \mathrm{g} / \mathrm{mL}$ streptomycin (Gibco), $2 \mathrm{mM} \mathrm{L-}$ Glutamine (Gibco) and $0.2 \mathrm{mM}$ L-ascorbic acid 2-phosphate (ASAP, Sigma-Aldrich). The samples in $\mathrm{BM}$ were then incubated at $37^{\circ} \mathrm{C}$ in humid atmosphere with $5 \% \mathrm{CO}_{2}$ for $1,4,7$ and 14 days. The protein amount of in the medium was measured with a QuantiPro ${ }^{\mathrm{TM}}$ BCA Assay Kit (Sigma, The Netherlands) following the manufacturer's guidelines. The absorbance signal was measured with a spectrophotometer (Anthos Zenyth 3100, Anthos Labtec Instruments $\mathrm{GmbH}$, Salzburg, Austria) at $562 \mathrm{~nm}$, and the protein content in the solutions was calculated via a calibration curve prepared using standard BSA solutions. The protein content was expressed as mean \pm SD.

Additionally, the proteins adsorbed onto the materials were quantified. Briefly, all samples were washed three times with PBS, then $0.1 \mathrm{~mL}$ of RIPA buffer (Thermo scientific) was added to each sample and kept at $4^{\circ} \mathrm{C}$ for 10 minutes. Thereafter, the amount of protein detached from the ceramics into RIPA buffer was measured with QuantiPro ${ }^{\text {TM }}$ BCA Assay Kit as described above.

\subsubsection{Ion release of TCP, TCP-C and TCP-Au materials}

TCP, TCP-C and TCP-Au materials were immersed (in triplicates; $\mathrm{n}=3$ ) in $1 \mathrm{~mL}$ of $\mathrm{BM}$ and incubated at $37^{\circ} \mathrm{C}$ in humid atmosphere with $5 \% \mathrm{CO}_{2}$ for $1,4,7$ and 14 days to monitor ion exchanges between the samples and BM. Ca and P ion concentrations in the culture medium were measured with QuantiChromTM Ca assay kit (BioAssay, USA) and phosphoWorkTM Colorimetric P Assay Kit (AATBioquest, USA), respectively, following the manufacturer's guidelines. Absorbance measurements were performed with spectrophotometer at $620 \mathrm{~nm}$. Both Ca and $\mathrm{P}$ ion content were expressed as mean \pm SD.

\subsubsection{Preparation of smooth glass, glass-C and glass-Au materials}

Carbon- and gold-coated glass slides with a diameter of $11 \mathrm{~mm}$ (Thermo Scientific ${ }^{\mathrm{TM}}$ cover slides) were used to investigate the possible effect of carbon and gold on cellular behavior of 
hBMSCs on smooth surfaces. The same coating settings used for TCP discs were applied on glass slides, which were also sterilized in the same manner (paragraph 2.1.1).

\subsubsection{Cell culture}

\subsubsection{Isolation and expansion of hBMSCs}

Bone marrow aspirates $(20 \mathrm{~mL}$ ) were obtained from a donor (69 years old, female) with written informed consent. In brief, aspirates were resuspended using $20 \mathrm{G}$ needles, plated at a density of $5 \times 10^{5}$ cells $/ \mathrm{cm}^{2}$ and cultured in proliferation medium containing BM and basic fibroblasts growth factor (bFGF, $1 \mathrm{ng} / \mathrm{mL}$ Instruchemie, the Netherlands). Cells were expanded at $37^{\circ} \mathrm{C}$ in a humid atmosphere with $5 \% \mathrm{CO}_{2}$, medium was refreshed twice a week and cells were subcultured until $80 \%$ confluence. Cells at passage 3 were then cultured on experimental surfaces.

\subsubsection{Cell culture on experimental materials}

Cells were cultured on the experimental surfaces in BM without supplementation of osteogenic-inducing factors. All experimental materials were placed in the wells of 48-well plates (for TCP-based materials) or 24-well plates (for glass-based materials) with the coated surface upside and pre-treated with BM for at least 4 hours. A cell suspension of $1 \mathrm{~mL}$ (for TCP-based materials) or $2 \mathrm{~mL}$ (for glass-based materials) at a seeding density of either 25,000 cells $/ \mathrm{mL}$ or 5,000 cells $/ \mathrm{mL}$ (only for cell morphology) were seeded onto experimental surfaces.

During the experiment, the BM was refreshed every 3 days. To observe cell morphology, samples were harvested at day 4; for cell proliferation, TCP samples were harvested at day 1 , 4, 7 and 14 while glass samples were harvested at day 1, 4 and 7 (data is not available at day 14 , since a full layer of cells detached from the surface of glass related materials); for alkaline phosphate (ALP) activity assay, TCP samples were harvested at day 4, 7 and 14 while glass samples were harvested at day 4 and 7; for bone related gene expression, TCP samples were harvested at day 4,7 and 14 .

\subsubsection{Cell attachment and distribution}

Samples of all TCP-based materials were harvested, and cells were fixated with $10 \%$ neutral formalin and stained using $1 \%$ methylene blue, followed by washing with distilled water until 
transparency of the washing liquid. The attachment and distribution of hBMSCs on the discs were observed with a light microscope (LM; E600, Nikon, Japan) equipped with KL 1500 LCD camera.

\subsubsection{Cell morphology}

Cell morphology was viewed via fluorescent microscopy. The samples were harvested 4 days after cell seeding and fixated for 30 minutes in $4 \%$ paraformaldehyde. Then, they were washed with PBS and permeabilized in $0.25 \%$ Triton-X 100 in PBS. F-actin was subsequently stained using phalloidin-AF488 (LifeTechnologies) and nuclei were stained using DAPI (Sigma Aldrich). Thereafter, the samples were thoroughly rinsed with PBS and cell morphology on TCP-based samples was observed using a BD Pathway system (BD Pathway 435, BD Biosciences), while cells on glass slide-related samples were imaged using a fluorescence microscope (Nikon Eclipse E600).

\subsubsection{Cell proliferation and ALP activity assays}

Cell proliferation was measured with a DNA assay (CyQuant Cell Proliferation Assay kit, Sigma), while ALP activity was measured using a CDP-star assay kit (Roche). After rinsing three times with PBS, the samples (TCP-based materials: $n=3$ per material per time point, three rounds of experiments; glass-based materials: $n=5$ per material per time point) were stored at $-20^{\circ} \mathrm{C}$ until further use. Prior to analysis, $300 \mu \mathrm{L}$ lysis buffer (prepared according to the manufacturer's instructions) was applied to each sample, followed by three cycles of freezing $\left(-20^{\circ} \mathrm{C}\right)$ and thawing (room temperature).

Prior to starting total DNA measurements, $100 \mu \mathrm{L}$ cell lysate was incubated in the dark at room temperature for 1 hour. Afterwards, $100 \mu \mathrm{L}$ CyQuant GR dye was mixed with each cell lysate at room temperature for 15 minutes and fluorescence was measured using a spectrophotometer (Victor, Perkin Elmer) at an excitation of $480 \mathrm{~nm}$ and emission wavelength of $520 \mathrm{~nm}$. The DNA content of cells was then calculated with a standard DNA curve.

ALP activity was measured according to the manufacturer's instructions. In brief, $10 \mu \mathrm{L}$ cell lysate and $40 \mu \mathrm{L}$ of CDP star substrate mixture were incubated for 20 min after which luminescence was measured with a spectrophotometer (Victor, Perkin Elmer). ALP activity was normalized for DNA content. 


\subsubsection{Bone-related genes expression}

Bone-related gene expression was evaluated with a quantitative real-time polymerase chain reaction (qPCR) assay. RNA isolation was performed using Trizol reagent (Invitrogen) and Nucleospin RNA isolation kit (Macherey-Nagel Gmbh \& Co.) following the manufacturer's instructions. Total RNA was measured using a NanoDrop spectrophotometer (Nanodrop technologies). The RNA was used to synthesize complementary DNA (cDNA) with iScriptcDNA Synthesis kit (Bio-Rad) according to the manufacturer's instructions. PCR analysis was performed with the Bio-Rad real-time PCR system (Bio-Rad, Hercules) on markers for ALP, collagen type I (Col I), OCN, and OPN, with beta-2 microglobulin (B2M) as the house-keeping gene for normalization. Primer sequences for ALP, Col I, OCN, OPN, and $\mathrm{B} 2 \mathrm{M}$ are listed in Table 1. The relative amounts of target genes normalized by B2M were calculated with the $2^{-\Delta C T}$ method where $\Delta C_{T}=C_{T, \text { Target }}{ }^{-} C_{T, B 2 M}$. All experiments were done on samples in triplicate $(n=3)$.

Table 1. Primer sequences for osteogenic related genes

\begin{tabular}{lll}
\hline Gene & Forward primer & Reverse primer \\
\hline OCN & TGAGAGCCCTCACACTCCTC & CGCCTGGGTCTCTTCACTAC \\
OPN & CTCCATTGACTCGAACGACTC & CAGGTCTGCGAAACTTCTTAGAT \\
ALP & ACAAGCACTCCCACTTCATC & TTCAGCTCGTACTGCATGTC \\
Col-I & AGGGCCAAGACGAAGACATC & AGATCACGTCATCGCACAACA \\
B2M & GACTTGTCTTTCAGCAAGGA & ACAAAGTCACATGGTTCACA \\
\hline
\end{tabular}

\subsubsection{Statistical analysis}

Multiple comparisons were performed with two-way analysis of variance (ANOVA) followed by Bonferroni post-test comparisons. $p$ values smaller than 0.05 were considered as statistically significant. 
a

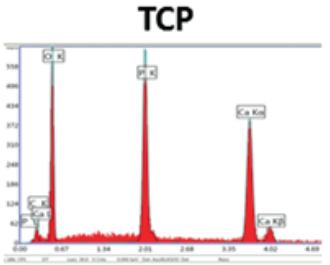

TCP-C
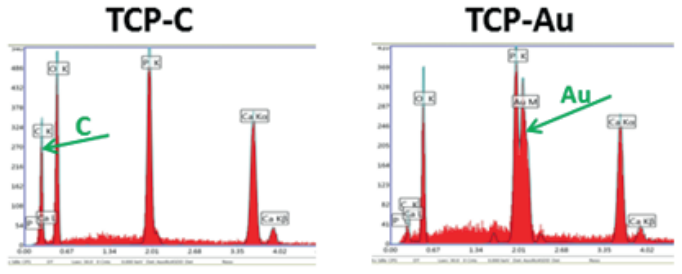

b

TCP
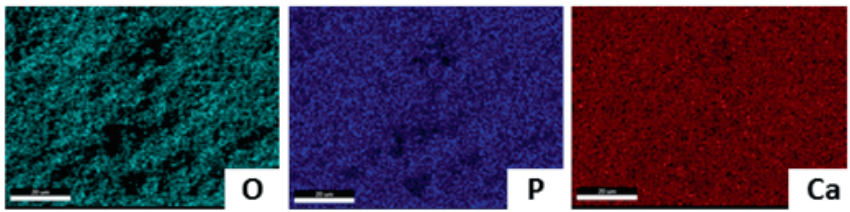

TCP-C
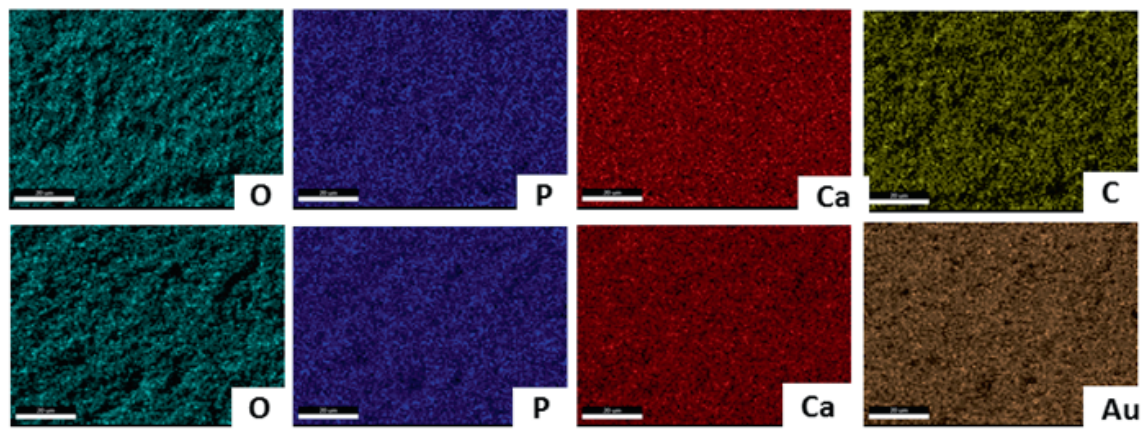

TCP-Au

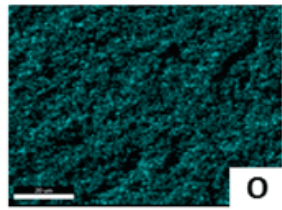

Figure 1. Chemistry of TCP, TCP-C and TCP-Au surfaces. EDX analysis shows (a) the presence of the elements and (b) elemental mappings. (Scale bar $=20 \mu \mathrm{m}$ )

\subsection{Results}

\subsubsection{Physico-chemical characterization of TCP, TCP-C and TCP-Au surfaces}

The EDX results of TCP, TCP-C and TCP-Au surface showed the presence of $\mathrm{Ca}, \mathrm{P}$ and $\mathrm{O}$ elements at all surfaces (Figure 1a). An additional peak for carbon was present in the TCP-C spectrum, while an extra peak for gold was observed from the analysis of TCP-Au surface. Elemental mapping demonstrated homogenous distribution of carbon on TCP-C surface (Figure $1 b$, TCP-C, C) and gold on TCP-Au surface (Figure 1b, TCP-Au, Au).

The surface structure of TCP-based samples was observed using SEM (Figure 2a) and similar surface structures were observed for TCP, TCP-C, and TCP-Au. The surfaces presented submicron-scaled grains and nano-scaled pores. Additionally, AFM morphological analysis (Figure $2 \mathrm{~b}$ ) and quantitated surface roughness (Figure $2 \mathrm{c}$ ) showed similarity for TCP, TCP-C, 
and TCP-Au, with submicron-scale roughness values for both surface roughness average (range Ra: $406.33 \pm 34.38$ - $482.8 \pm 73.02 \mathrm{~nm}$ ) and root mean square (range Rq: $503 \pm 29.11$ $590 \pm 90.13 \mathrm{~nm})$.

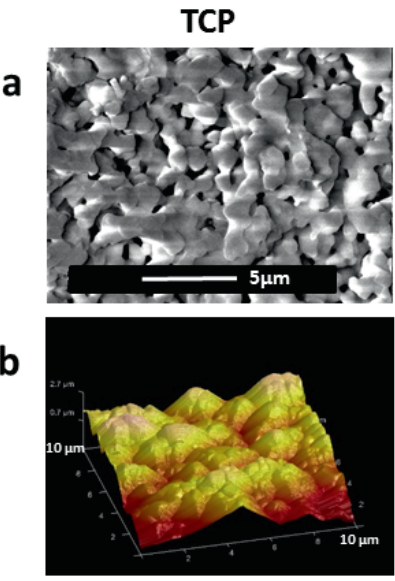

C

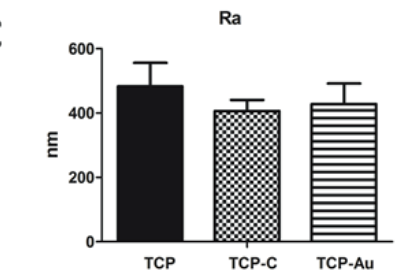

TCP-C
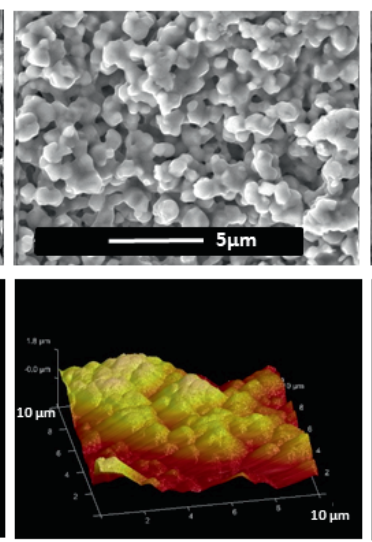

TCP-Au
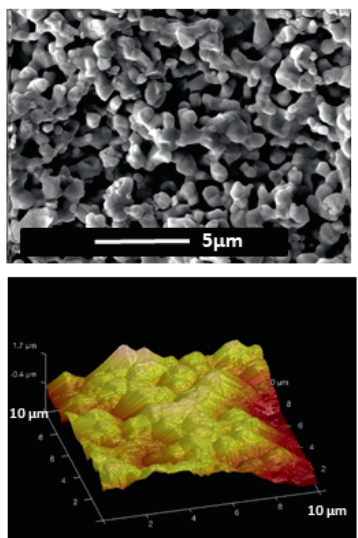

$\mathrm{Rq}$

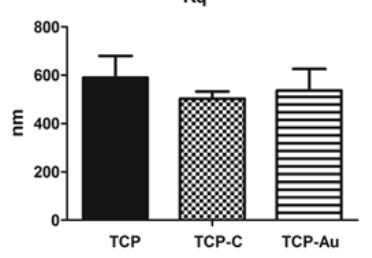

Figure 2. Surface topography and roughness of TCP, TCP-C and TCP-Au surface. (a) The surface structure using SEM; (b) the surface structure using AFM and (c) surface roughness average ( $R a$ ) and root mean square $(R q)$ of TCP, TCP-C and TCP-Au surface. No significant differences were seen for either $R a$ or $R q$ among the analyzed surfaces.

\subsubsection{Protein adsorption by TCP, TCP-C and TCP-Au surfaces}

The total amount of proteins in BM after soaking TCP-based materials and the control group (i.e. BM only) is illustrated in Figure 3a, while the amount of proteins adsorbed onto TCPbased materials is presented in Figure 3b. The amount of proteins (around $3000 \mu \mathrm{g}$ ) in the medium did not change with time for any of the TCP-based materials and no significant differences were observed among the TCP-based materials and the control group. Protein adsorption to the TCP-based materials was similar for all experimental groups, ranging from 24.14 to $30.61 \mu \mathrm{g}$ per disc in the period from 1 to 14 days after initiation of soaking in BM 
(Figure $3 b$ ). Comparing the results shown in Figure $3 a$ and $3 b$, only $\sim 1 \%$ of the protein initially present in BM was adsorbed onto TCP-based materials, explaining why no significant changes of protein content in medium were observed upon immersion of the TCP-based materials.

a

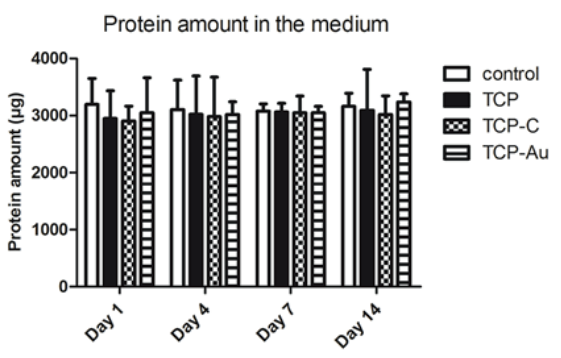

b

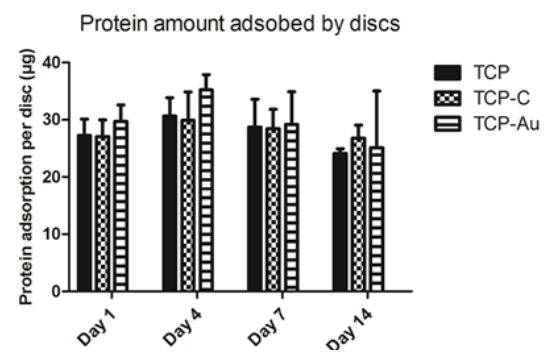

C
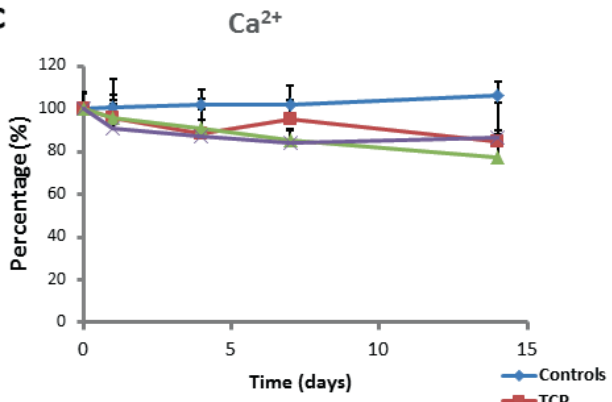

d

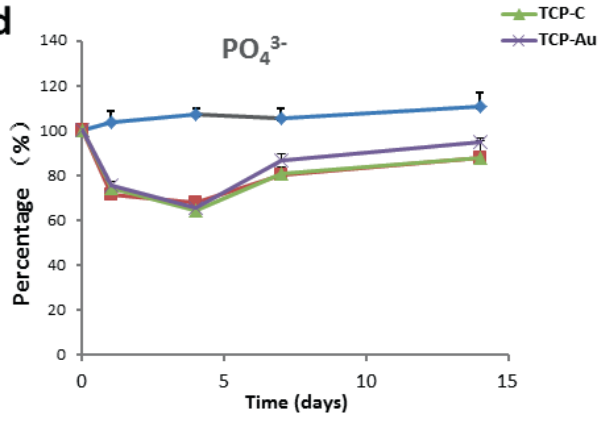

Figure 3. (a) Protein concentrations in the medium 1, 4, 7 and 14 days after immersion of TCP, TCP-C and TCP-Au disCs; (b) Amount of proteins adsorbed onto materials, (c) calcium and (d) phosphate ions concentration changes in culture medium after soaking the three TCP based discs up to 14 days (Either calcium or phosphate ions concentration in control medium on day 0 is $100 \%)$.

\subsubsection{Calcium and phosphate ion release}

Figures $3 c$ and $3 d$ depict the variations of $\mathrm{Ca}$ and $\mathrm{P}$ ions concentrations in BM containing TCP, TCP-C and TCP-Au discs. One may observe that the changing trends of both ions were similar for all materials. Ca ion concentration slightly decreased during the experimental period, while the $\mathrm{P}$ ion concentration decreased till day 4 , and increased thereafter. Both $\mathrm{Ca}$ and $\mathrm{P}$ ions concentrations were always lower as compared to the control group (BM only), indicating that TCP, TCP-C and TCP-Au discs adsorbed ions from BM. 


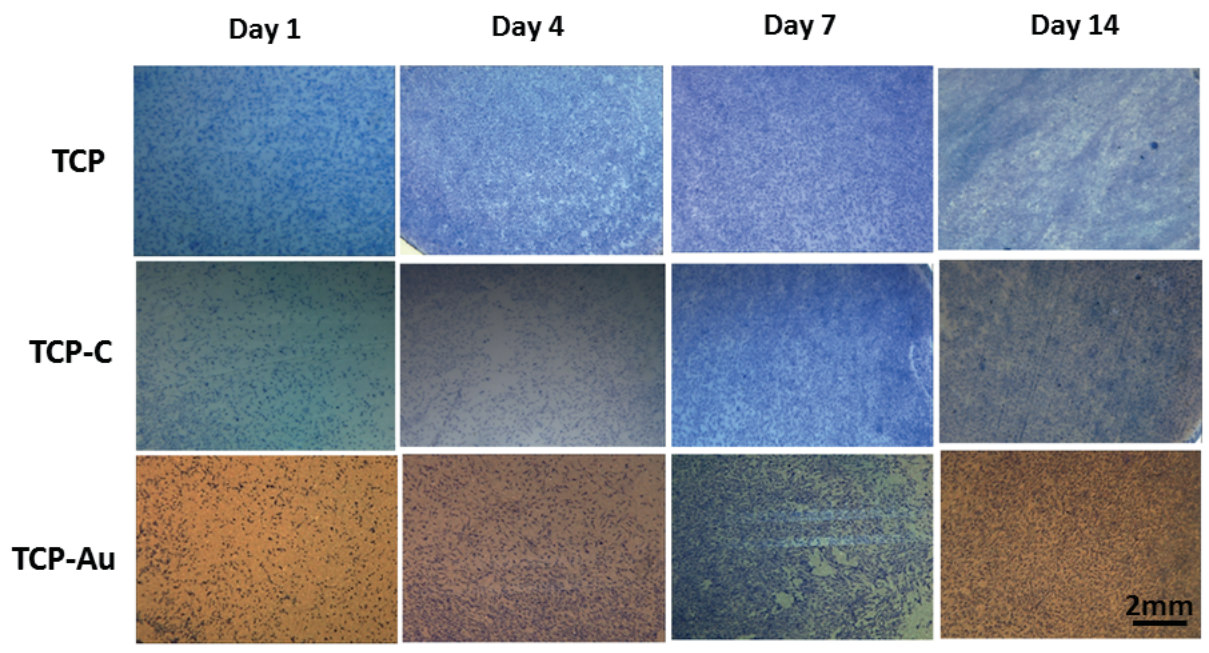

Figure 4. Methylene blue staining and steromicroscopic observation of hBMSCs cultured on TCP, TCP-C and TCP-Au surfaces for 1, 4, 7 and 14 days.

TCP

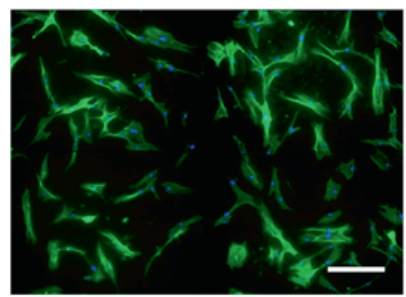

TCP-C

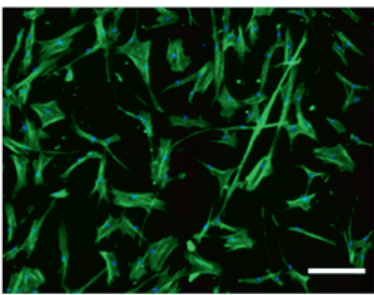

TCP-Au

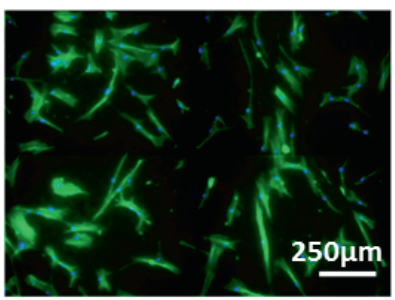

Figure 5. Morphology of cells on TCP, TCP-C and TCP-Au surface under fluorescent imaging after immunochemical staining of DAPI (blue, nucleus) and phalloidin (green: actin skeleton) 4 days after cell seeding.

\subsubsection{The effect of surface chemistry on cellular behavior}

\subsubsection{Cell attachment and growth}

As viewed by stereomicroscopy after methylene blue staining (Figure 4), hBMSCs were homogeneously distributed over the surface of all TCP-based materials at one day after cell seeding. Further, an apparent increase of cell density with culture time was observed for all experimental groups. 


\subsubsection{Cell morphology}

The morphology of hBMSCs cultured on the all TCP-based materials at 4 days after low cell density seeding $(5,000 \mathrm{cell} / \mathrm{mL})$ was observed after immunochemical staining with phalloidin and DAPI (Figure 5). Cell morphology on all the TCP-based materials was similar, showing spread and elongated cells.

\subsubsection{Proliferation and ALP activity of hBMSCs}

Proliferation and osteogenic differentiation of hBMSCs on TCP, TCP-C and TCP-Au surfaces were analyzed using DNA and ALP activity assays (Figures $6 a$ and 6b). Cells seeded at a density of 25,000 cells $/ \mathrm{ml}$ were cultured for up to 14 days. Cell cultures on all three TCPbased materials displayed an increase in DNA content over time without any significant difference among the surfaces (Figure 6a). ALP activity of hBMSCs kept similar from day 4 to day 7 and increased at day 14 for all the TCP-based materials without significant differences among the three TCP-based materials.

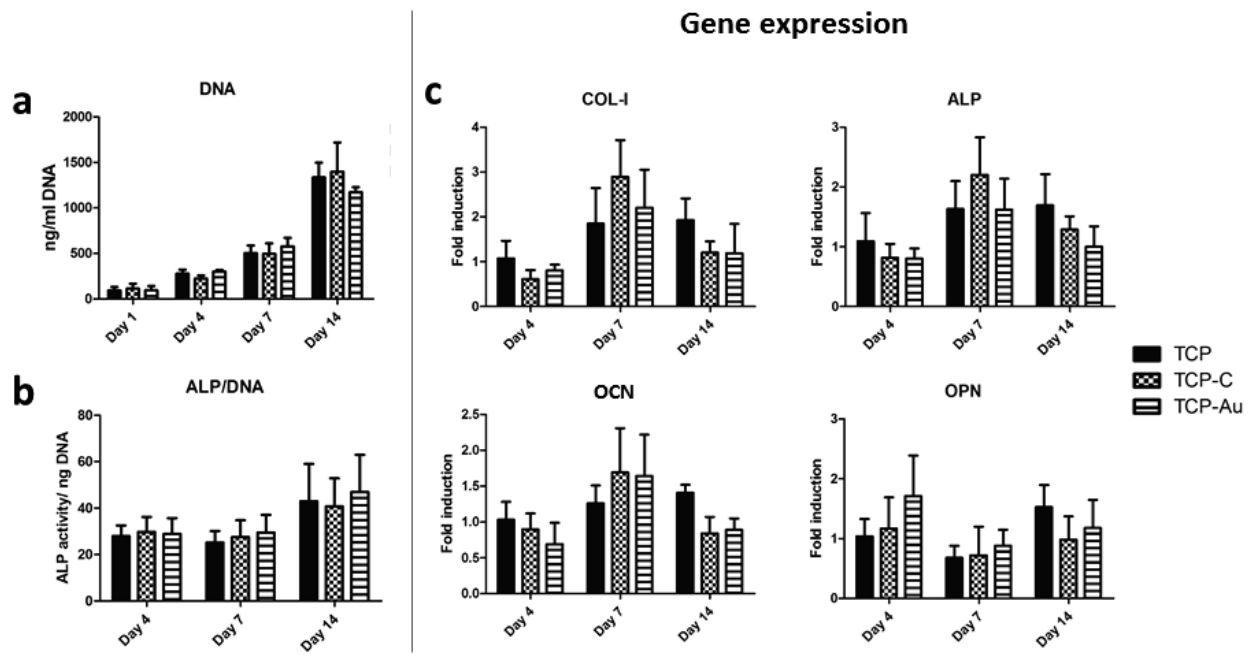

Figure 6. Proliferation, osteogenic differentiation and gene expression of hBMSCs on TCP, TCP-C and TCP-Au surfaces over time. (a) DNA quantification; (b) ALP activity (normalized to DNA amount) and (c) Osteogenic gene expression profiles (normalized to the B2M housekeeping gene). 
a

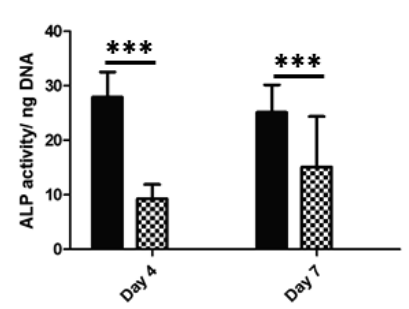

b

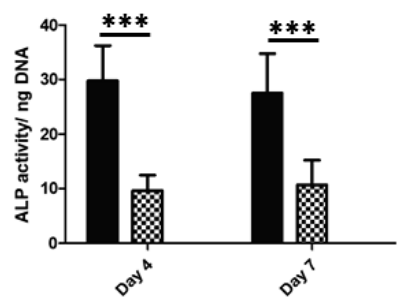

C

Au coating

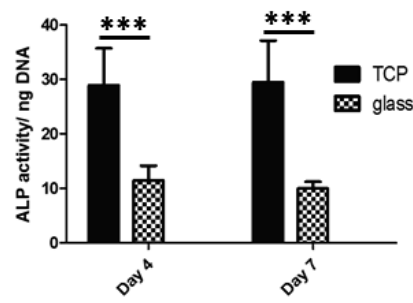

Figure 7. The importance of surface topography on osteogenic differentiation of hBMSCs. Significant higher ALP activity (normalized to DNA amount) of hBMSCs cultured on TCP based materials than glass based materials after 4 and 7 days (a) No coating; (b) $C$ coating and (c) Au coating.

\subsubsection{Gene expression}

ALP, COL I, OCN and OPN gene expression was determined by qPCR assays (Figure 6c). Col-I, ALP, OCN gene expression of hBMSCs cultures on all three TCP-based materials was increase from day 4 to day 7 and decrease at day 14, while OPN gene expression kept similar over time until day 14 for all the TCP-based materials. It is notable that, for all the gene expression, no significant differences was observed among the three TCP-based materials.

\subsubsection{The important role of surface topography in osteogenic differentiation of hBMSCs}

To determine the important role of surface topography on osteogenic differentiation of hBMSCs, ALP activity of cells cultured on submicron scaled TCP-based materials and smooth glass-based materials were compared after culturing for 4 and 7 days. Significantly higher ALP activity ( $p<0.001$ at day $4, p<0.01$ at day 7$)$ was observed on submicron scaled TCPbased materials than smooth glass-based materials at both day 4 and day 7 , irrespective of surface chemistry (Figure 7).

\subsection{Discussion}

It is well documented that the surface structure of CaP ceramics is crucial to induce osteogenesis in vivo. In an ectopic implantation model, CaP ceramics without micropores (i.e. pores $<10 \mathrm{um}$ ) did not form bone while those having micropores did [10-12, 32]. It has been shown that the osteoinductive potential increases with the microporosity of CaP ceramics [13] and that within similar microporosity, only the CaP ceramic with submicron scaled pores 


\section{Chapter 4}

triggered bone formation $[14,15]$. The instructive role of surface structures was also seen in vitro: without additional growth factors, osteogenic differentiation of hBMSCs was enhanced on submicron scaled surface structure of $\mathrm{CaP}$ ceramics as compared to those micron scaled surface structure [15]. The submicron structure of CaP ceramics not only affected the osteogenic differentiation of MSCs, but also regulates osteoclast formation from monocytes and their functionality in vitro [17]. The surface structure of CaP ceramics not only altered the topographical cue but also the soluble chemical cues via protein adsorption and ion exchange $[14,15]$. Thus, the instructive function of surface structure in inductive bone formation by CaP ceramics could be considered as the function of both the soluble chemical and the specific topographic cues. In the current study we showed that the topographical cue of CaP ceramics could directly instruct the osteogenic response.

To distinguish the role of surface topography from soluble chemical cues, we coated submicron scaled TCP ceramic discs and smooth glass slides with either carbon or gold. With the homogenous presence of thin carbon and gold coatings on the TCP ceramic disc surface (Figure 1b), the chemistry of the surface to which hBMSCs reacted was altered (Figure 1a), but the surface structure was kept unchanged (Figures $2 \mathrm{a}, 2 \mathrm{~b}$ and $2 \mathrm{c}$ ). The coating technique allowed uniform covering of the outer surface of TCP discs, without altering the submicron scaled grains present in the core of discs. As a result, the protein adsorption of the ceramics was not significantly changed by the coating (Figure $3 b$ ) and, thus, the amount of proteins left in the culture medium was the same for all TCP-based materials (Figure 3a). For the same reason, the ion exchanges of $\mathrm{Ca}$ and $\mathrm{P}$ kept the same for all TCP-based materials (Figure 3c). Since the same settings were applied to coat smooth glass slides, no changes in surface roughness can be expected; because of the much smaller surface area of the glass slides (approximately $0.94 \mathrm{~cm}^{2}$ for a glass slide of $\varnothing 11 \mathrm{~mm}$ and $1680 \mathrm{~cm}^{2}$ for a TCP disc of $\varnothing 9 \times 1 \mathrm{~mm}$, with a weight of $0.12 \pm 0.01 \mathrm{~g}$ and specific surface area of $1.4 \mathrm{~m}^{2} / \mathrm{g}$ [15]), changes of protein content, and $\mathrm{Ca}$ and $\mathrm{P}$ ion concentrations in culture medium containing glass-based materials are not expected.

The presence of carbon and gold was not harmful to the cells, which attached and grew on both coated and non-coated materials (Figure 4, 6a, S1a and S1b). In particular, no influence of the coatings on cell proliferation was seen in either TCP-based (Figure 6a) or glass-based 
materials (Figure S1b). Taking the ALP activity (normalized to DNA content) as an early osteogenic marker, carbon and gold coatings did not show their influence on osteogenic differentiation of hBMSCs on either TCP-based (Figure 6b) or glass-based (Figure S1c) materials. Additionally, both coatings did not show any effect on hBMSCs at the genes level: no differences could be noticed among the TCP-based materials regarding the gene expressions of COL I, ALP, OCN and OPN (Figure 6c). However, a difference of osteogenic differentiation potential between TCP-based and glass-based materials could be clearly seen. At day 4 and 7, the ALP activity of hBMSCs on TCP-based materials was almost three times higher than on glass-based materials (Figure 7).

The internal comparison among TCP-based and glass-based materials could exclude the possible function of surface chemical changes on cell activities. The presence of carbon or gold coatings chemically altered the surface to which the cells respond. Considering the case of glass-based materials, the surface chemistry changed due to presence of the coatings and of the various proteins that adsorbed onto their surfaces. In relation to TCP-based materials, next to the chemical alterations induced by the coatings and the protein adsorption, the surface mineralization could have a role as well. Given the bioactive nature of CaP ceramics, they may favored the formation of biological apatite onto their own submicron scaled surface [15]. Because of the bioinert nature of carbon and gold, the formation of apatite on the coated surface to which the cells react would be inhibited. Thus, if surface chemical changes have a role in cells' behavior, differences should be seen. However, the presence of carbon or gold on either TCP-based or glass-based materials did not cause any difference in cell proliferation and osteogenic differentiation of hBMSCs.

The comparison between TCP-based and glass-based materials pointed out the importance of surface topography on osteogenic differentiation of cells. In particular, the osteogenic differentiation was enhanced on TCP-based materials as compared to glass ones (Figures $6 \mathrm{~b}$ and S1c). Given the facts that TCP-based materials have rough surfaces (Figure 2) while those glass-based have smooth surfaces, the results agree with the general finding that a rougher surface enhances osteogenic differentiation of MSCs [33]. However, to conclude that the surface topography physically drove the osteogenic response of cells to osteoinductive $\mathrm{CaP}$ ceramics, the role of chemical changes in culture medium containing TCP-based materials should be excluded. As shown in Figure 3c, the $\mathrm{Ca}$ and P ion concentrations in culture medium decreased when TCP-based materials were soaked, while the 


\section{Chapter 4}

protein amount negligibly changed. It was demonstrated that increased concentrations of $\mathrm{Ca}$ and $\mathrm{P}$ ions in the medium have a positive influence on the osteogenic differentiation of cells [28]. If the ions in the medium played roles in the current study, higher osteogenic differentiation should be seen in the case of glass-based materials because of the higher concentrations of $\mathrm{Ca}$ and $\mathrm{P}$ ions in the medium as compared to TCP-S based materials. However, inferior osteogenic differentiation of hBMSCs was observed on glass-based materials. Therefore, in this study the enhanced osteogenic differentiation of hBMSCs on TCP-based materials could not be resulted from the chemical changes of the culture medium.

Specific biological response are often attributed to chemical cues, while increasing evidences show the biological functions of physical cues [30,31]. In this study, when coating a smooth surface, carbon did not have any osteogenic role (Figure S1c), while it could induce the differentiation of hBMSCs towards osteogenic phenotypes once rough surfaces were created using carbon nano tubes $[34,35]$. Similarly, gold coating on smooth glass surface had no osteogenic function (Figure 7c), while gold nanoparticles promoted osteogenic differentiation of MSCs [36, 37]. These latter observations further clarify that surface topography has an osteogenic function on MSCs. It is generally thought that, via the socalled contact guidance process, MSCs can adapt their morphology according to the surface topography and undergo differentiation [38]. The cell morphology of hBMSCs observed on TCP-based (Figure 5) and glass-based materials (Figure S1a) agrees with the general finding that elongated and more spread cells undergo osteogenic differentiation [38, 39].

By using TCP-based materials with submicron surface structure and glass-based materials with smooth surface, we showed the crucial role of surface topographical cue in the cellular osteogenic response. In particular, a rougher surface appeared to be more osteogenic in vitro as shown in the current study and more osteoinductive in vivo as reported in our previous study [18], while it should be mentioned that the scale of the surface structure matters in the phenomenon of material-directed osteoinduction. Together with the previous finding, a suitable surface structure is suggested to be at the submicron/nanometer scale. Both a smooth surface and a too rough one (i.e. above the micron scale) resulted in nonosteoinductive materials [14-17]. 
By keeping the soluble chemical cues constant in the medium, in this study we showed the osteogenic function of topographical cues in CaP ceramics with submicron scale surface structure. However, the mechanism behind topographic-driven osteogenic differentiation is not fully understood as yet. Moreover, in the in vivo experimental settings osteoinductive and non-osteoinductive CaP ceramics differed not only in their surface topography but also in the biochemical factors they could concentrate from the surrounding body fluids via protein adsorption. The role of biochemical factors in material-driven osteoinduction and the synergic function between topographical and soluble biochemical factors are nowadays still unknown.

\subsection{Conclusions}

Using an in vitro model of hBMSCs and via carbon and gold coatings of submicron scale surface structured TCP ceramic discs and smooth glass slides, we excluded the possible influence of chemical cues (i.e. surface chemistry changes, proteins and ions exchange with the medium) on CaP ceramic- driven osteoinduction. The function of the surface topographical cues in cell's osteogenic response to CaP ceramics was clearly shown in the cross comparison done between TCP-based and glass-based materials: the submicron scaled surface structure could physically instruct osteogenic differentiation of MSCs in vitro. 
a glass glass-C glass-Au
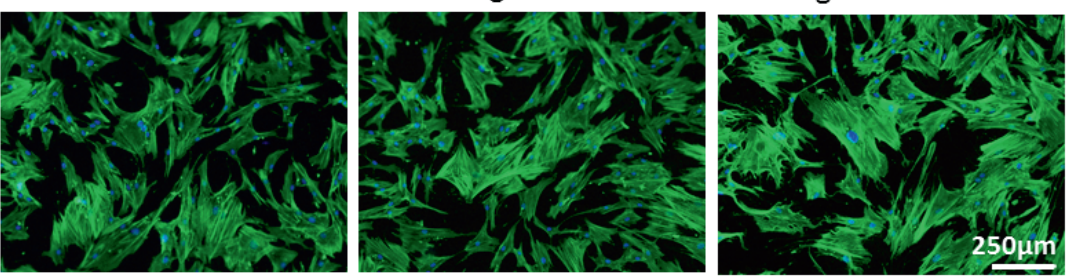

b
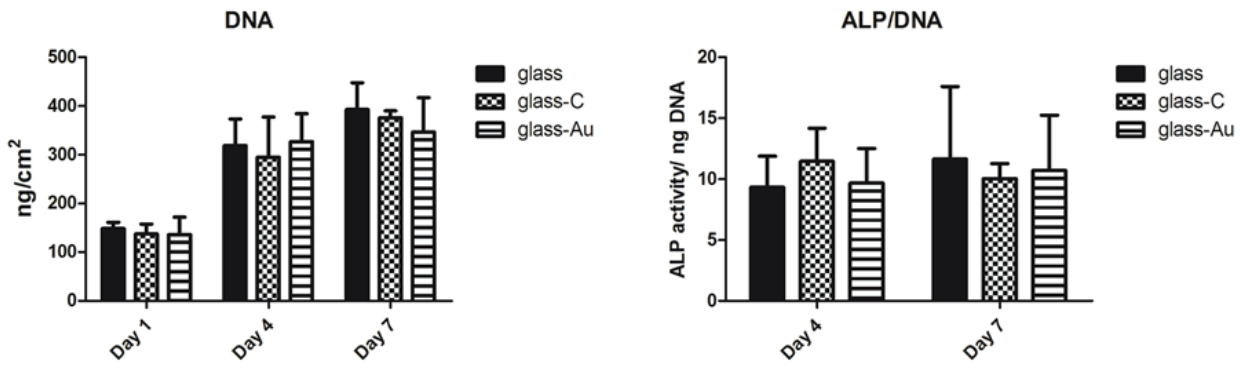

Supplementary Figure 1. Cell activities of hBMSCs on glass, glass-C and glass-Au smooth surfaces. (a) cell morphology at day 4 under fluorescent microscopy with immunostainings of DAPI (blue: nucleus) and phalloidin (green: actin skeleton); (b) DNA quantification and ALP activity (normalized to DNA amount) over time. 


\section{References}

[1] Jarcho M. Calcium phosphate ceramics as hard tissue prosthetics. Clinical orthopaedics and related research. 1981;157:259-78.

[2] Damien CJ, Parsons JR. Bone graft and bone graft substitutes: a review of current technology and applications. Journal of Applied Biomaterials. 1991;2:187-208.

[3] Blokhuis TJ, Termaat MF, den Boer FC, Patka P, Bakker FC, Henk JTM. Properties of calcium phosphate ceramics in relation to their in vivo behavior. Journal of Trauma-Injury, Infection, and Critical Care. 2000;48:179.

[4] Yuan H, Fernandes H, Habibovic P, De Boer J, Barradas A, De Ruiter A, et al. Osteoinductive ceramics as a synthetic alternative to autologous bone grafting. Proceedings of the National Academy of Sciences of the United States of America. 2010;107:13614-9.

[5] Samavedi S, Whittington AR, Goldstein AS. Calcium phosphate ceramics in bone tissue engineering: a review of properties and their influence on cell behavior. Acta Biomaterialia. 2013;9:8037-45.

[6] Habibovic P, Kruyt MC, Juhl MV, Clyens S, Martinetti R, Dolcini L, et al. Comparative in vivo study of six hydroxyapatite - based bone graft substitutes. Journal of Orthopaedic Research. 2008;26:1363-70.

[7] Barradas AM, Yuan H, van Blitterswijk CA, Habibovic P. Osteoinductive biomaterials: current knowledge of properties, experimental models and biological mechanisms. European cells \& materials. 2011;21:407-29.

[8] Wang H, Zhi W, Lu X, Li X, Duan K, Duan R, et al. Comparative studies on ectopic bone formation in porous hydroxyapatite scaffolds with complementary pore structures. Acta biomaterialia. 2013;9:8413-21.

[9] Ripamonti U, Crooks J, Kirkbride A. Sintered porous hydroxyapatites with intrinsic osteoinductive activity: geometric induction of bone formation. 1999.

[10] Habibovic P, Sees TM, van den Doel MA, van Blitterswijk CA, de Groot K. Osteoinduction by biomaterialsphysicochemical and structural influences. Journal of Biomedical Materials Research Part A. 2006;77:747-62.

[11] Yamasaki H, Sakai H. Osteogenic response to porous hydroxyapatite ceramics under the skin of dogs. Biomaterials. 1992;13:308-12.

[12] Yuan H, Kurashina K, de Bruijn JD, Li Y, De Groot K, Zhang X. A preliminary study on osteoinduction of two kinds of calcium phosphate ceramics. Biomaterials. 1999;20:1799-806.

[13] Coathup MJ, Hing KA, Samizadeh S, Chan O, Fang YS, Campion C, et al. Effect of increased strut porosity of calcium phosphate bone graft substitute biomaterials on osteoinduction. Journal of Biomedical Materials Research Part A. 2012;100:1550-5.

[14] Davison N, Luo X, Schoenmaker T, Everts V, Yuan H, Barrère-de Groot F, et al. Submicron-scale surface architecture of tricalcium phosphate directs osteogenesis in vitro and in vivo. European cells \& materials. 2013;27:281-97.

[15] Zhang J, Luo X, Barbieri D, Barradas A, de Bruijn JD, van Blitterswijk CA, et al. The size of surface microstructures as an osteogenic factor in calcium phosphate ceramics. Acta biomaterialia. 2014;10:3254-63.

[16] Davison NL, Gamblin A-L, Layrolle P, Yuan H, de Bruijn JD, Barrère-de Groot F. Liposomal clodronate inhibition of osteoclastogenesis and osteoinduction by submicrostructured beta-tricalcium phosphate. Biomaterials. 2014;35:5088-97.

[17] Davison NL, ten Harkel B, Schoenmaker T, Luo X, Yuan H, Everts V, et al. Osteoclast resorption of betatricalcium phosphate controlled by surface architecture. Biomaterials. 2014;35:7441-51.

[18] Zhang J, Barbieri D, ten Hoopen $H$, de Bruijn JD, van Blitterswijk CA, Yuan H. Microporous calcium phosphate ceramics driving osteogenesis through surface architecture. Journal of Biomedical Materials Research Part A. 2015:3:1188-99

[19] Urist MR, Huo YK, Brownell AG, Hohl WM, Buyske J, Lietze A, et al. Purification of bovine bone morphogenetic protein by hydroxyapatite chromatography. Proceedings of the National Academy of Sciences of the United States of America. 1984;81:371.

[20] Habibovic P, Yuan H, Van Der Valk CM, Meijer G, Van Blitterswijk CA, De Groot K. 3D microenvironment as essential element for osteoinduction by biomaterials. Biomaterials. 2005;26:3565-75.

[21] Mizrahi O, Sheyn D, Tawackoli W, Kallai I, Oh A, Su S, et al. BMP-6 is more efficient in bone formation than BMP-2 when overexpressed in mesenchymal stem cells. Gene therapy. 2013;20:370-7.

[22] Luther G, R Wagner E, Zhu G, Kang Q, Luo Q, Lamplot J, et al. BMP-9 induced osteogenic differentiation of mesenchymal stem cells: molecular mechanism and therapeutic potential. Current gene therapy. 2011;11:229-40. 
[23] Song IH, Caplan AI, Dennis JE. In vitro dexamethasone pretreatment enhances bone formation of human mesenchymal stem cells in vivo. Journal of orthopaedic research. 2009;27:916-21.

[24] Khoshniat S, Bourgine A, Julien M, Weiss P, Guicheux J, Beck L. The emergence of phosphate as a specific signaling molecule in bone and other cell types in mammals. Cellular and Molecular Life Sciences. 2011;68:20518.

[25] Dvorak MM, Siddiqua A, Ward DT, Carter DH, Dallas SL, Nemeth EF, et al. Physiological changes in extracellular calcium concentration directly control osteoblast function in the absence of calciotropic hormones. Proceedings of the National Academy of Sciences of the United States of America. 2004;101:5140-5.

[26] Chai YC, Roberts SJ, Schrooten J, Luyten FP. Probing the osteoinductive effect of calcium phosphate by using an in vitro biomimetic model. Tissue Engineering Part A. 2010;17:1083-97.

[27] Kanatani M, Sugimoto T, Fukase M, Fujita T. Effect of elevated extracellular calcium on the proliferation of osteoblastic MC3T3-E1 cells: its direct and indirect effects via monocytes. Biochemical and biophysical research communications. 1991;181:1425-30.

[28] Barradas AM, Fernandes HA, Groen N, Chai YC, Schrooten J, van de Peppel J, et al. A calcium-induced signaling cascade leading to osteogenic differentiation of human bone marrow-derived mesenchymal stromal cells. Biomaterials. 2012;33:3205-15.

[29] Reynolds JL, Joannides AJ, Skepper JN, McNair R, Schurgers LJ, Proudfoot D, et al. Human vascular smooth muscle cells undergo vesicle-mediated calcification in response to changes in extracellular calcium and phosphate concentrations: a potential mechanism for accelerated vascular calcification in ESRD. Journal of the American Society of Nephrology. 2004;15:2857-67.

[30] Fu J, Wang YK, Yang MT, Desai RA, Yu X, Liu Z, et al. Mechanical regulation of cell function with geometrically modulated elastomeric substrates. Nature methods. 2010;7:733-6.

[31] Mitragotri S, Lahann J. Physical approaches to biomaterial design. Nat Mater. 2009;8:15-23.

[32] Habibovic P, Yuan H, van den Doel M, Sees TM, van Blitterswijk CA, de Groot K. Relevance of Osteoinductive Biomaterials in Critical - Sized Orthotopic Defect. Journal of Orthopaedic Research. 2006;24:86776.

[33] Faia-Torres AB, Guimond-Lischer S, Rottmar M, Charnley M, Goren T, Maniura-Weber K, et al. Differential regulation of osteogenic differentiation of stem cells on surface roughness gradients. Biomaterials. 2014;35:902332.

[34] Nayak TR, Jian L, Phua LC, Ho HK, Ren Y, Pastorin G. Thin films of functionalized multiwalled carbon nanotubes as suitable scaffold materials for stem cells proliferation and bone formation. ACS nano. 2010;4:771725.

[35] Baik KY, Park SY, Heo K, Lee KB, Hong S. Carbon nanotube monolayer cues for osteogenesis of mesenchymal stem cells. small. 2011;7:741-5.

[36] Yi C, Liu D, Fong C-C, Zhang J, Yang M. Gold nanoparticles promote osteogenic differentiation of mesenchymal stem cells through p38 MAPK pathway. ACS nano. 2010;4:6439-48.

[37] Kawazoe N, Chen G. Gold nanoparticles with different charge and moiety induce differential cell response on mesenchymal stem cell osteogenesis. Biomaterials. 2015;54:226-36.

[38] Gao L, McBeath R, Chen CS. Stem Cell Shape Regulates a Chondrogenic Versus Myogenic Fate Through Rac1 and N - Cadherin. Stem Cells. 2010;28:564-72.

[39] Kumar G, Tison CK, Chatterjee K, Pine PS, McDaniel JH, Salit ML, et al. The determination of stem cell fate by 3D scaffold structures through the control of cell shape. Biomaterials. 2011;32:9188-96. 


\section{Chapter 5}

\section{Cells responding to surface structure of calcium phosphate ceramics for bone regeneration}

Jingwei Zhang ${ }^{a, b}$, Lanying Sun ${ }^{c}$, Xiaoman Luo ${ }^{d, e}$, Davide Barbieri ${ }^{d}$, Joost D. de Bruijn ${ }^{d, e, f}$, Clemens A. van Blitterswijk ${ }^{\mathrm{a}, \mathrm{g}}$, Lorenzo Moroni ${ }^{\mathrm{a}, \mathrm{g}}$, Huipin Yuan ${ }^{\mathrm{a}, \mathrm{d}, \mathrm{g}, \mathrm{h}}$ *

a Department of Tissue Regeneration, MIRA Institute for Biomedical Technology and Technical Medicine, University of Twente, P.O. Box 217, 7500AE Enschede, The Netherlands

${ }^{b}$ Key Laboratory of Advanced Technologies of Materials, Ministry of Education, School of Materials Science and Engineering, Southwest Jiaotong University, Chengdu, P.R. China

${ }^{c}$ Oral Implantology Center, Stomotology Hospital of Jinan, Jingliu Road 101, 250001, Jinan, China

${ }^{d}$ Xpand Biotechnology BV, Bilthoven, The Netherlands

e Department of Biomaterials Science and Technology, MIRA Institute for Biomedical Technology and Technical Medicine, University of Twente, P.O. Box 217, 7500AE Enschede, The Netherlands

${ }^{f}$ School of Engineering and Materials Science (SEMS), Queen Mary University of London, E1 4NS London, United Kingdom

${ }^{g}$ Complex Tissue Regeneration department, MERLN Institute for Technology Inspired Regenerative Medicine, Maastricht University, Universiteitsingel 40, 6229 ER, The Netherlands

${ }^{h}$ College of Physical Science and Technology, Sichuan University, Chengdu, China 


\begin{abstract}
Surface structure largely affects the inductive bone forming potential of calcium phosphate (CaP) ceramics in ectopic sites and bone regeneration in critical-sized bone defects. Surfacedependent osteogenic differentiation of bone marrow stromal cells (BMSCs) partially explained the improved bone forming ability of submicron surface structured CaP ceramics. In this study, we investigated the possible influence of surface structure on the response of other bone related cells that may potentially participate in the process of improved bone formation in CaP ceramics. The response of HBVP (pericytes), C2C12 (osteogenic inducible cells), MC3T3-E1 (osteogenic precursors), SV-HFO (pre-osteoblasts), MG63 (osteoblasts) and SAOS-2 (mature osteoblasts) to the surface structure was evaluated in terms of cell proliferation, osteogenic differentiation and gene expression. The cells were cultured on tricalcium phosphate (TCP) ceramics with either micron-scaled surface structure (TCP-B) or submicron-scaled surface structure (TCP-S) for up to 14 days, followed by DNA, ALP and qPCR gene assays. HBVP were not sensitive to surface structure with respect to cell proliferation and osteogenic differentiation but had downregulated angiogenesis-related gene expression (i.e. VEGF) on TCP-B. Without additional osteogenic inducing factors, submicron-scaled surface structure enhanced ALP activity and osteocalcin gene expression of C2C12 cells, favored the proliferation of MC3T3-E1, MG63 and SAOS-2, and increased ALP activity of MC3T3-E1 and SV-HFO. The results herein indicate that cells with osteogenic potency (either osteogenic inducible cells or osteogenic cells) could be sensitive to surface structure and respond to osteoinductive submicron-structured CaP ceramics in the way of cell proliferation, ALP production or osteogenic gene expression, which favor bone regeneration.
\end{abstract}

\title{
Keywords:
}

Bone regeneration, Calcium phosphate ceramic, Surface structure, Cell proliferation, Osteogenic

differentiation 


\subsection{Introduction}

Calcium phosphate (CaP) ceramics have been used in various orthopedic and dental applications as bone regenerative scaffolds due to their excellent biocompatibility and osteoconductivity [1-5]. In addition to osteoconductivity, which allows a passive support for bonding osteogenesis on the surface, in the past two decades, CaP ceramics were reported to have also the ability to induce bone formation when implanted ectopically without the addition of osteogenic-inducing factors and/or cells if they present specific physicochemical properties [6-11]. Having the capacity to form bone in ectopic sites, osteoinductive CaP ceramics enhanced bone repair in non-critical sized defects [12], could repair critical-sized defects in goat iliac wings [13] and performed equally to autologous bone and BMP-2 in critically sized defects in sheep iliac wings [14].

Among the physicochemical properties relevant to material-driven osteoinduction, the surface structure of CaP ceramics was found crucial $[6,7]$. It has been reported that hydroxyapatite (HA) ceramics with micropores are osteoinductive after subcutaneous [6] and intramuscular [7] implantations in dogs, while no bone formed in those lacking of micropores. The osteoinductive potential of silicon substituted HA increased with the microporosity in an ovine ectopic model [15]. When considering ceramics with the same microporosity, a clearer difference in inductive bone formation could be generated via varying the dimension of surface structure. A tricalcium phosphate (TCP) ceramic with a submicron scaled surface structure has been shown osteoinductive, while TCP presenting a micron scaled surface structure has not following an intramuscular implantation in dogs [16, 17] and subcutaneous implantation in FVB mice $[18,19]$.

From no bone to form bone, inductive bone formation in materials in ectopic sites must have a crucial step of osteogenic differentiation of stem cells. Being multipotent, BMSCs are often used as a stem cell model for osteogenic differentiation [20-22]. Recent findings showed that BMSCs could migrate from the bone marrow to the implantation site via blood circulation and participate in ectopic bone formation of osteoinductive CaP ceramics [23]. When BMSCs were cultured on TCP ceramics in absence of osteogenic-inducing factors, we have already shown that their osteogenic differentiation was enhanced on ceramics with submicron scaled surface structure as compared to those having micron scaled surface 
structure [16]. These results partially explained why material-driven osteoinduction could happen in ectopic sites.

Next to BMSCs, other cell types showing their stemness and capacity of osteogenic differentiation may be involved in ectopic bone formation as well. Pericytes, which have physiological roles in angiogenesis and tissue homeostasis [24], showed their multipotency as they could differentiate into myofibroblast, smooth muscle cells and osteoblasts [25]. As BMSCs did, pericytes (e.g. vascular pericytes) underwent osteogenic differentiation in vitro and were involved ectopic calcification and osteogenesis in vivo [26, 27]. Myoblasts could be another inducible cell source involved in bone formation following an intramuscular implantation. Although satellite cell-derived primary myoblasts isolated from adult skeletal muscle fibers of mouse are more readily differentiated into osteogenic and adipogenic lineages than primary myoblasts [28], the mouse myoblast cell line C2C12 is an often-used inducible cell system to evaluate the osteogenic function of growth factors [29, 30]. These inducible cells (e.g. BMSCs, pericytes and myoblasts) may respond to osteoinductive CaP ceramic surface structure to differentiate into osteogenic progenitors, thereafter undergoing maturation step by step from pre-osteoblasts to mature osteoblasts finally forming ectopic bone.

In addition to inducible cells, osteogenic cells at various developmental stages responded differently to the surface structure [31]. It is possible that the ectopic bone formation in osteoinductive CaP ceramics would be further enhanced by the responses of osteogenic cells differentiated from inducible cells to surface structure. In the osseous sites, the osteogenic cells at various mature stages are present as well. Therefore, the enhanced bone regeneration by osteoinductive CaP ceramics would be resulted from both the inducible cells and osteogenic cells which are sensitive to surface structure.

The objective of the current study was to address the possible involvement of pericytes and myoblasts in inductive bone formation and the possible influence of the surface structure on the osteogenesis of osteogenic cells at various maturation stages. To do so, we compared HBVP (pericytes), C2C12 (osteogenic inducible cells), MC3T3-E1 (osteogenic precursors), SVHFO (pre-osteoblasts) [32, 33], MG63 (osteoblasts) and SAOS-2 (mature osteoblasts) on two 
well-established TCP ceramic surface structures with respect to cell proliferation, osteogenic differentiation and gene expression.

\subsection{Materials and methods}

\subsubsection{Preparation of TCP ceramic granules}

TCP ceramic granules were prepared as described previously [16]. In brief, two TCP powders were synthesized by adding, at different rates, $\mathrm{H}_{3} \mathrm{PO}_{4}$ solution into $\mathrm{Ca}(\mathrm{OH})_{2}$ suspension with a $\mathrm{Ca} / \mathrm{P}$ molecular ratio of 1.50. TCP ceramics with micron scaled (TCP-B) or submicron scaled (TCP-S) surface structure were prepared from TCP powders using $\mathrm{H}_{2} \mathrm{O}_{2}$ (1\%) and wax granules as porogen. The green bodies were subsequently sintered at $1050^{\circ} \mathrm{C}$ (TCP-S) or $1100^{\circ} \mathrm{C}$ (TCP-B) for 8 hours and ceramic granules with size of 1-2 mm were collected, cleaned, dried and steam sterilized at a pressure of $1-1.1$ bar $\left(120^{\circ} \mathrm{C}\right)$ for $30 \mathrm{~min}$.

As previously characterized TCP-B and TCP-S [16], both TCP ceramics contain the same chemistry of $\beta$-TCP. As regards surface properties, TCP-S has a grain size of $0.99 \pm 0.20 \mu \mathrm{m}$ and a micropore size of $0.65 \pm 0.25 \mu \mathrm{m}$, while TCP-B has larger grain and micropore dimensions, being $3.08 \pm 0.52 \mu \mathrm{m}$ and $1.58 \pm 0.65 \mu \mathrm{m}$ respectively. TCP-S has larger specific surface area $\left(1.2 \mathrm{~m}^{2} \mathrm{~g}^{-1}\right)$ than TCP-B $\left(0.8 \mathrm{~m}^{2} \mathrm{~g}^{-1}\right)$ as well. The total porosity of TCP-S and TCP-B are comparable, being 70 and $72 \%$ respectively. Further, they also have similar microporosity (22\% and $23 \%$ for TCP-S and TCP-B respectively).

\subsubsection{Cells}

A total of six types of cells, i.e. HBVP, C2C12, MC3T3-E1, MG63, SV-HFO and SAOS-2, were used in this study. HBVP (Human Brain Vascular Pericytes, ScienCell) and C2C12 cells (murine myoblast cell line, ScienceCell) were cultured in Dulbecco's modified Eagle's medium (DMEM, Life Technologies) containing 10\% fetal bovine serum (FBS, Lonza), $100 \mathrm{U} / \mathrm{mL}$ penicillin (Life Technologies) and $100 \mu \mathrm{g} / \mathrm{mL}$ streptomycin (Life Technologies). MC3T3-E1 cells (subclone 14) were cultured in $\alpha$-MEM, supplemented with 10\% FBS, 2 mM L-Glutamine, $0.01 \mathrm{mM}$ sodium pyruvate (Sigma), $10 \mathrm{mM}$ bGP (Sigma) and $0.2 \mathrm{mM}$ ASAP. SV-HFO (human pre-osteoblastic cell) was cultured in phenol-free a-MEM (Life Technologies) containing 10\% FBS, $100 \mathrm{U} / \mathrm{mL}$ penicillin, $100 \mu \mathrm{g} / \mathrm{ml}$ streptomycin (Life Technologies), $20 \mathrm{mM} \mathrm{HEPES} \mathrm{(Sigma)} \mathrm{and} \mathrm{pH-}$ adjusted to 7.5. MG63 (osteosarcoma cell line, ATCC) and SAOS2 (osteosarcoma cell line, 
ATCC) were incubated in $\alpha$-MEM supplemented with $10 \%$ FBS, $100 \mathrm{U} / \mathrm{mL}$ penicillin and 100 $\mu \mathrm{g} / \mathrm{mL}$ streptomycin, $2 \mathrm{mM}$ L-Glutamine and $0.2 \mathrm{mM}$ ASAP.

Cell cultures were maintained at $37{ }^{\circ} \mathrm{C}$ and $5 \% \mathrm{CO}_{2}$. Mediums were refreshed twice a week, and further sub-culturing or cryopreservation upon confluence were performed in order to get a sufficient number of cells for the tests.

\subsubsection{Cell culture on TCP ceramics}

\subsubsection{Cell seeding}

Before cell seeding, ceramic granules (100 mg per sample) were loaded into 25-well suspension plates and pretreated with cell-specific medium for at least four hours. Thereafter, $150 \mu \mathrm{L}$ of cell suspension was seeded on the materials. The seeding density varied with cell types because of the different growth rates of the cells. For $\mathrm{C} 2 \mathrm{C} 12$ and MC3T3-E1, 200,000 cells were seeded per sample, while 500,000 cells were used for HBVP, MG63, SAOS2 and SV-HFO.

\subsubsection{Cell culturing on TCP ceramics}

Four hours after cell seeding, $2 \mathrm{~mL}$ of culture medium was added and finally cells were cultured with medium refreshment twice a week.

Only $\mathrm{C} 2 \mathrm{C} 12$ cells were cultured in mediums with and without rhBMP-2 to study the possible synergic function of the surface structure and rhBMP-2 (Hangzhou Biodoor Biothechonology Co., $250 \mathrm{ng} / \mathrm{mL}$ ) in osteogenic differentiation. All the other cells (i.e. HBVP, MC3T3-E1, MG63, SAOS2 and HFO) were cultured with their specific mediums without any additive. Samples were harvested at day 1, 4, 7 and 14 for the DNA assay, while for alkaline phosphatase (ALP) activity and gene expression assays the samples were harvested at day 4, 7 and 14 respectively.

\subsubsection{DNA and ALP activity assays}

Cell proliferation was evaluated using a DNA assay (CyQuant Cell Proliferation Assay kit, Sigma, the Netherlands) and ALP activity was analyzed with a CDP-star assay kit (Roche). Briefly, all samples were stored at $-20^{\circ} \mathrm{C}$ for at least 24 hours after rinsing thrice with PBS. One $\mathrm{mL}$ lysis buffer (prepared according to the manufacturer's instructions of CDP-star Assay 
kit instructions) was added to each sample, followed by three cycles of freezing at $-20^{\circ} \mathrm{C}$ and thawing at room temperature, to get lysates.

The cell lysate was then mixed in a $1: 1$ volume ratio with $1 \mathrm{mg} / \mathrm{mL}$ proteinase $\mathrm{K}$ (SigmaAldrich), $18.5 \mu \mathrm{g} / \mathrm{mL}$ iodoacetamide (Sigma Aldrich) and $1 \mu \mathrm{g} / \mathrm{mL}$ pepstatin A (Sigma Aldrich) in Tris/EDTA buffer (pH 7.6) and incubated for 16 hours at $56^{\circ} \mathrm{C}$. Total DNA amount was measured using the CyQuant ${ }^{\mathrm{TM}}$ DNA assay (Molecular Probes) according to the manufacturer's instructions and calculated through a standard DNA curve. Fluorescence was measured using a spectrophotometer (Victor, Perkin Elmer, excitation $480 \mathrm{~nm}$ \& emission $520 \mathrm{~nm})$.

To measure the relative ALP activity, CDP-star ${ }^{\circledR}$ assay kit (Roche) was used according to the manufacturer's protocol. Briefly, $40 \mu \mathrm{L}$ of CDP star substrate were incubated with $10 \mu \mathrm{L}$ cell lysate for $20 \mathrm{~min}$, and the luminescence was subsequently measured using a spectrophotometer. The ALP activity was then normalized to the DNA quantity per sample.

\subsubsection{Bone- and angiogenesis-related gene expression}

To investigate bone-related genes' expression profile of each cell type instructed by the surface structure of the different materials as well as the possible role of surface structure on the angiogenesis of pericytes, real-time polymerase chain reaction (PCR) assay was performed. After 4, 7 and 14 days, total RNA was isolated from each cell type cultured on the materials using TRIzol (Invitrogen) and the Nucleospin RNA isolation kit (MachereyNagelGmbh \& Co.) according to the manufacturer's protocol. RNA concentration was measured using a NanoDrop spectrophotometer (Nanodrop technologies, USA). The RNA was used to synthesize complementary DNA (cDNA) with an iScript cDNA Synthesis kit (BioRad) according to the manufacturer's instructions. The bone related markers ALP, osteocalcin (OCN) and osteopontin (OPN), the angiogenesis markers vascular endothelial growth factor (VEGF), VEGF receptor-2 (KDR) and platelet endothelial cell adhesion molecule (CD31) were measured with a Bio-Rad real-time PCR system (Bio-Rad, Hercules, CA, USA). Beta-2 microglobulin (B2M) was the human house-keeping gene and Glyceraldehyde 3phosphate dehydrogenase (GAPDH) was the mouse house-keeping gene. All the human primer sequences are listed in Table 1 while those for mouse are listed in Table 2. 
Table 1. Human primer sequence

\begin{tabular}{lll}
\hline Gene & Forward primer & Reverse primer \\
\hline OCN & TGAGAGCCCTCACACTCCTC & CGCCTGGGTCTCTTCACTAC \\
OPN & CTCCATTGACTCGAACGACTC & CAGGTCTGCGAAACTTCTTAGAT \\
ALP & ACAAGCACTCCCACTTCATC & TTCAGCTCGTACTGCATGTC \\
KDR & ACTTTGGAAGACAGAACCAAATTATCTC & TGGGCACCATTCCACCA \\
VEGF & AGGGCAGAATCATCACGAAGT & AGGTCTCGATTGGATGGCA \\
CD31 & TCTAGACCTCGCCCTCCACA & GAACGGTGTCTTCAGGTTGGTATTTCA \\
B2M & GACTTGTCTTTCAGCAAGGA & ACAAAGTCACATGGTTCACA \\
\hline
\end{tabular}

Table 2. Mouse primer sequence

\begin{tabular}{lll}
\hline Gene & Forward primer & Reverse primer \\
\hline OCN & CAGACCTAGCAGACACCATGAGG & AGGTCAGAGAGACAGAGCGCA \\
OPN & GATGCCACAGATGAGGACCTC & CTGGGCAACAGGGATGACAT \\
ALP & CTGGGCAACAGGGATGACAT & GGATCATCGTGTCCTGCTCAC \\
GAPDH & AACGACCCCTTCATTGAC & TCCACGACATACTCAGCAC \\
\hline
\end{tabular}

Data was analyzed using Bio-Rad iQ5 software. The relative amounts of target genes normalized by B2M or GAPDH were calculated by double delta $\mathrm{Ct}$ method, subsequently normalized to the gene expression level of each type of cells determined after four days cultured on TCP-B granules. All experiments were done in triplicate $(n=3)$.

\subsubsection{Statistical analysis}

All experiments were done in triplicate $(n=3)$. Multiple comparisons were performed with two way analysis of variance (ANOVA) followed by Bonferroni post-test comparisons. Statistically significant differences are marked by * for $p<0.05, * *$ for $p<0.01$, and $* * *$ for $p$ $<0.001$. 


\subsection{Results}

HBVP cells grew on TCP ceramic granules, as shown by the increase in DNA content over time (Figure 1a). The cells kept on growing slowly until day 7 on both TCP ceramics (Figure 1a), while no difference could be observed between TCP-B and TCP-S. ALP activity of HBVP was slightly down-regulated during time on both materials, while no differences between TCP-B and TCP-S were detected (Figure 1b).

DNA content

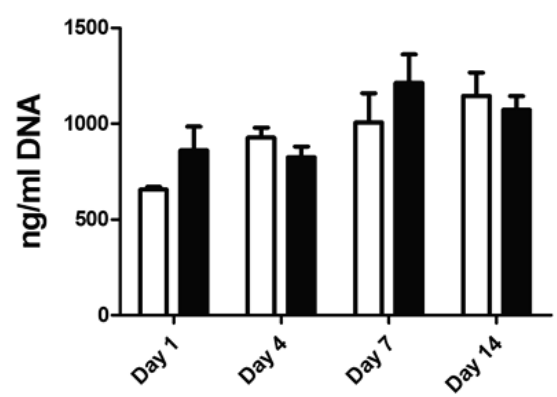

ALP activity

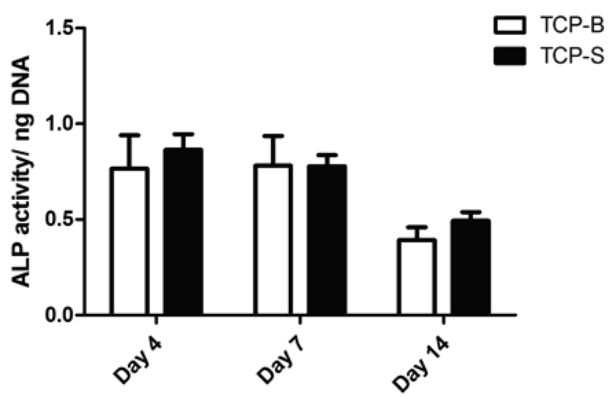

Figure 1. Proliferation (a) and osteogenic differentiation (b) of HBVP cultured on TCP ceramics over time.
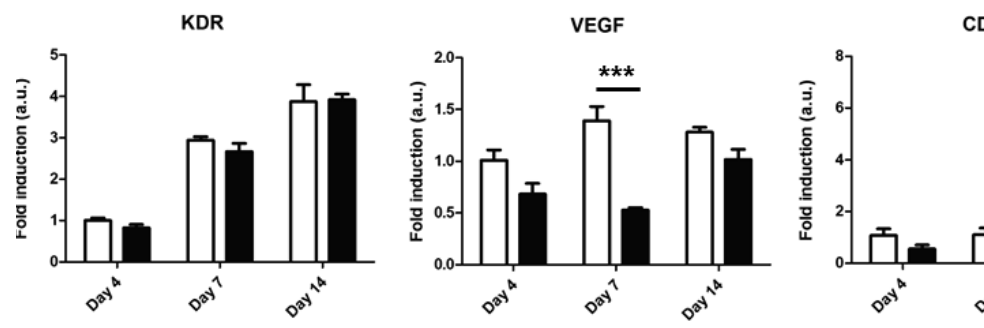

CD31

Figure 2. Angiogenesis gene expression of HBVP cultured on TCP-B and TCP-S over time.

The angiogenesis-related gene expression profile of HBVP cultured on TCP-B and TCP-S granules is illustrated in Figure 2. Time-dependent gene expression was seen with KDR and CD31. Material-dependent gene expression was not observed for the angiogenesis-related genes of KDR and CD31, while VEGF gene was up-regulated on TCP-B ceramic at day 7 compared to TCP-S $(\mathrm{p}<0.001)$. 
a
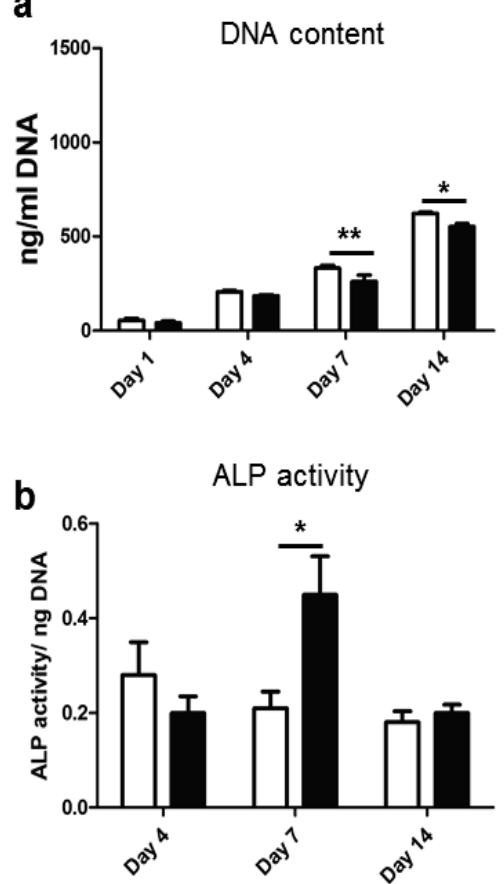

C Gene expression
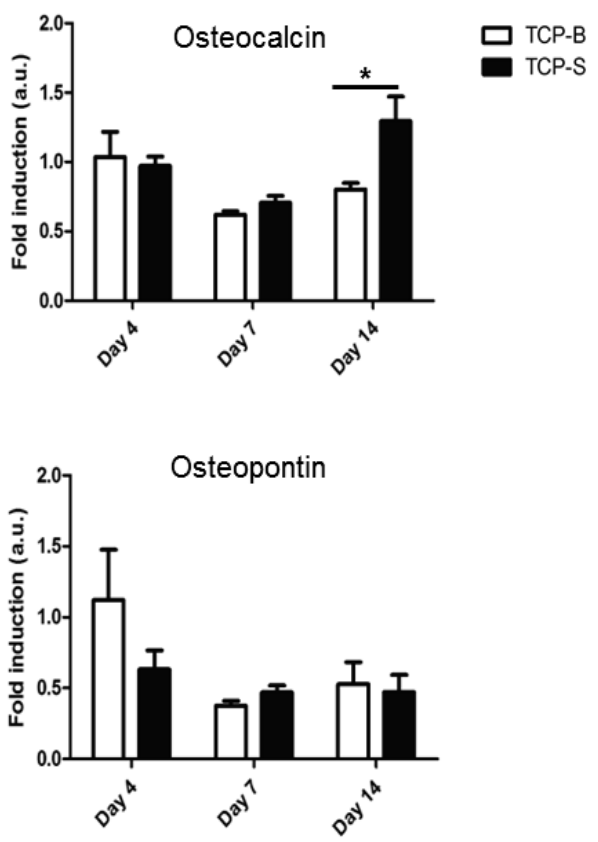

Figure 3. Proliferation (a), ALP activity (b) and gene expression (c) of $C 2 C 12$ cultured on TCP ceramics over time without rhBMP-2.

When cultured in the medium without rhBMP-2, C2C12 grew well on both TCP ceramics and increased in cell number over time (Figure 3a). When TCP-B and TCP-S were compared, no difference in cell amount was observed at days 1 and 4, while a significantly higher cell amount was detected on TCP-B than on TCP-S at days 7 and 14 (Figure 3a). ALP activity of C2C12 cells did not change considerably over time on TCP-B, while higher ALP activity was shown on TCP-S at day 7 compared to days 4 and day 14 (Figure 3b). Material-dependent ALP activity was seen between TCP-B and TCP-S at day 7 (Figure 3b), where a significant higher ALP activity was noted for TCP-S compared to TCP-B $(P<0.05)$. Osteocalcin gene expression was up-regulated on TCP-S compared to TCP-B at day 14 (Figure $3 c)(P<0.05)$, no significant changes of osteopontin was observed between C2C2 cells cultured on TCP-S and TCP-B. 
a

DNA content

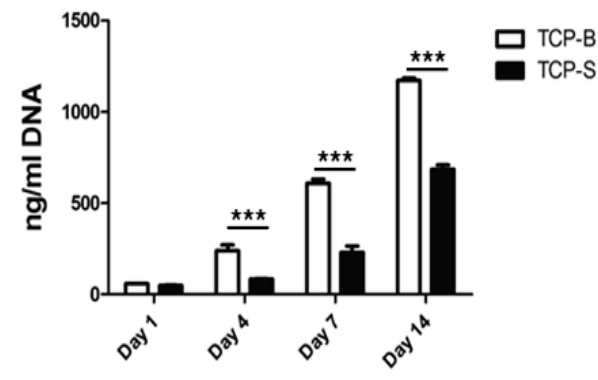

b

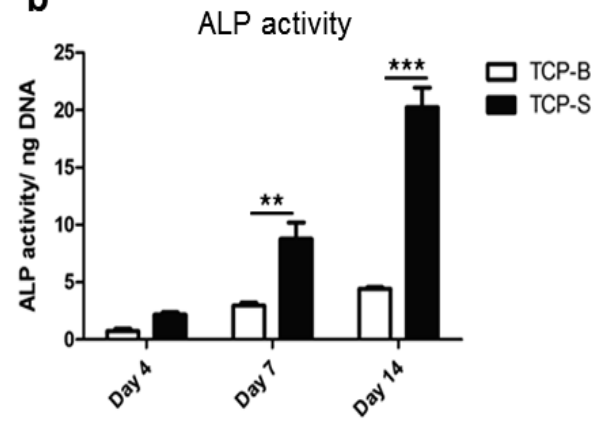

C Gene expression

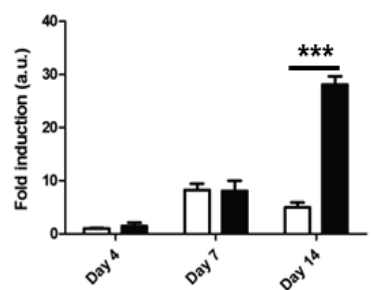

ALP

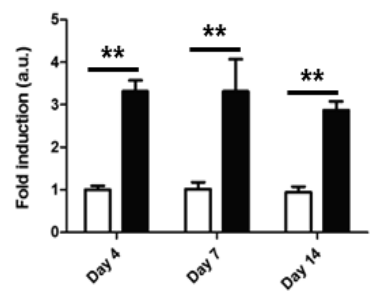

Osteocalcin

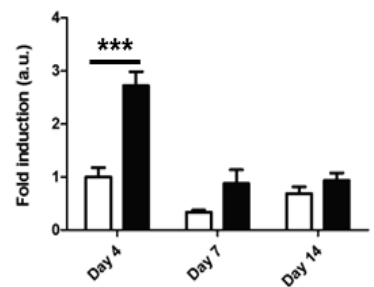

Osteopontin

Figure 4. Proliferation (a), ALP activity (b) and gene expression (c) of C2C12 cultured on TCP ceramics over time with rhBMP-2 (250ng/mL).

With the addition of rhBMP-2 in the culture medium $(250 \mathrm{ng} / \mathrm{mL})$, cell proliferation of $\mathrm{C} 2 \mathrm{C} 12$ was material-dependent. More cells could be detected on TCP-B at days 4, 7 and 14 than on TCP-S, as shown by the DNA content (Figure 4a). The ALP activity increased over time on both TCP-B and TCP-S, while higher ALP activity was observed on TCP-S than on TCP-B at all the time points (Figure 4b). ALP gene expression was significantly up-regulated on TCP-S at day 14 compared to TCP-B (Figure 4c). Osteocalcin gene expression was significantly higher on TCP-S at days 4, 7 and 14 compared to TCP-B (Figure 4c). Osteopontin gene expression also showed a significantly higher expression on TCP-S compared to TCP-B only at day 4 (Figure 4c). 


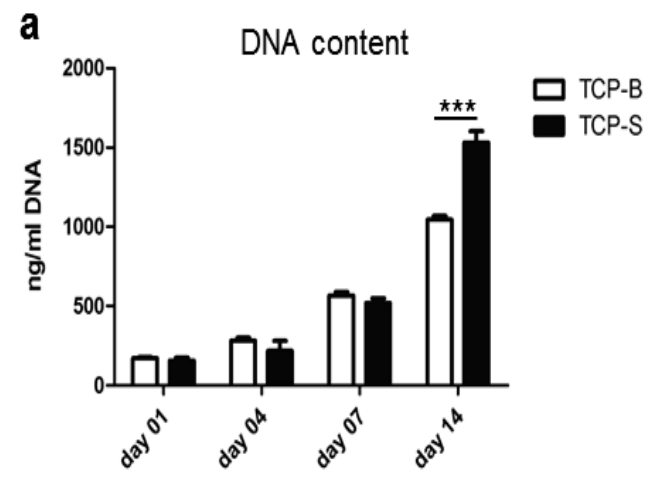

C Gene expression

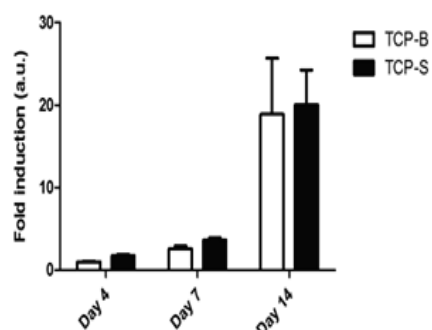

ALP

b

ALP activity
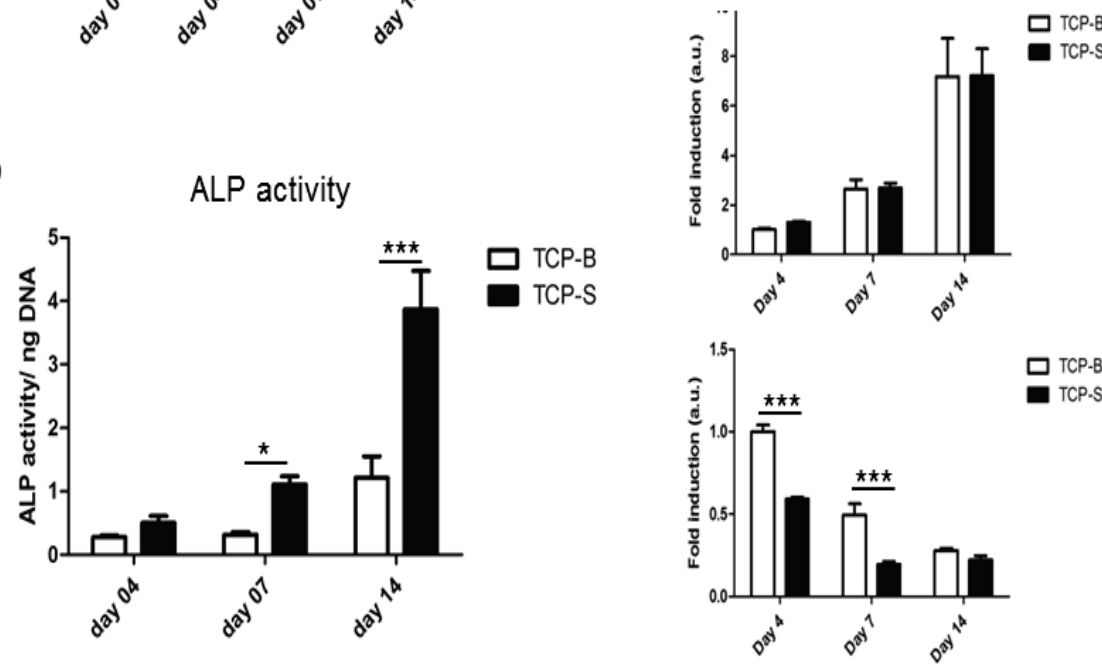

Osteocalcin

Osteopontin

Figure 5. Proliferation (a), ALP activity (b) and gene expression (c) of MC3T3-E1 cultured on TCP ceramics over time.

MC3T3-E1 cells proliferated during time on both TCP-B and TCP-S ceramics with an enhanced cell proliferation on TCP-S after a 14-day culture (Figure 5a). ALP activity of cells increased over time on both ceramics (Figure 5b) with a material-dependent difference. In particular, significantly higher ALP activity was detected on TCP-S than on TCP-B at days 7 and 14. Both ALP and osteocalcin genes were up-regulated over time, while materialdependent gene expression was not seen between TCP-B and TCP-S. Osteopontin gene expression decreased during time on both ceramics, and a material-dependent gene expression was seen with a significantly higher osteopontin gene expression on TCP-B than on TCP-S at days 4 and 7 (Figure $5 c$ ). 
a

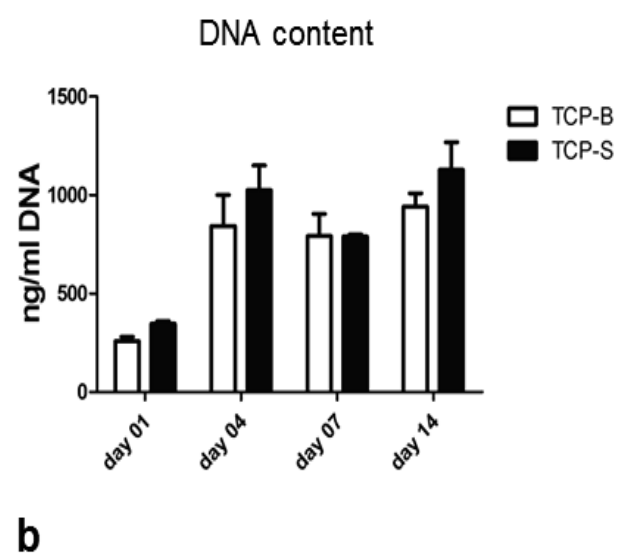

C Gene expression
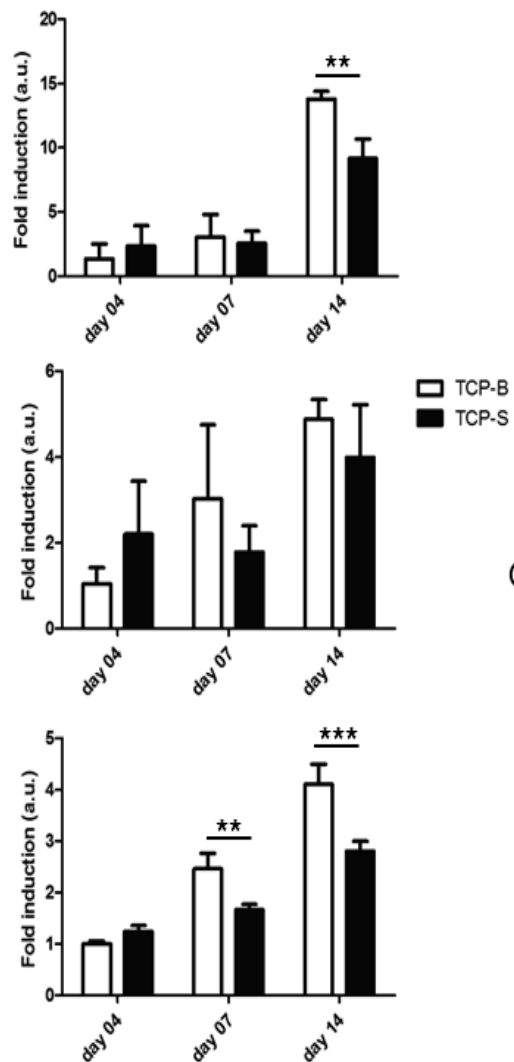

Osteocalcin

ALP<smiles>C1CC1</smiles>

Osteopontin

Figure 6. Proliferation (a), ALP activity (b) and gene expression (c) of SV-HFO cultured on TCP ceramics over time.

SV-HFO cells proliferated quickly from day 1 to 4 on both TCP-B and TCP-S, but did not proliferate much thereafter (Figure 6a). No material-dependent cell proliferation was seen (Figure 6a). A clear material-dependent ALP activity was seen: TCP-S supported a significantly higher ALP activity than on TCP-B at days 7 and 14 (Figure 6b). The gene expressions of $A L P$, osteocalcin and osteopontin increased during time (Figure 6c). Materialdependent gene expression was not observed in osteocalcin, while ALP gene was upregulated on TCP-B at day 14 and osteopontin gene was up-regulated on TCP-B at days 7 and day 14 as compared to TCP-S. 
MG63 kept on growing on both TCP-B and TCP-S over time (Figure 7a). A significantly higher cell proliferation was shown at day 14 on TCP-S than on TCP-B (Figure 7a). The ALP activity of MG63 cells decreased in a time-dependent manner on both TCP-B and TCP-S, and no difference was found between the two ceramics at any time point (Figure 7b). Osteopontin gene expression of MG63 was not detectable on either TCP-B or TCP-S, while the expression of ALP and osteocalcin genes was down-regulated during time with no influence of the materials (Figure 7c).

a

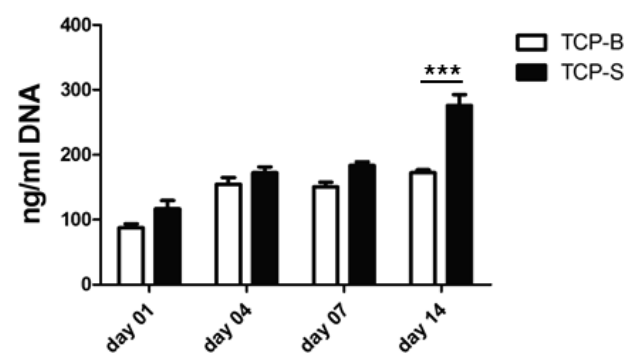

b

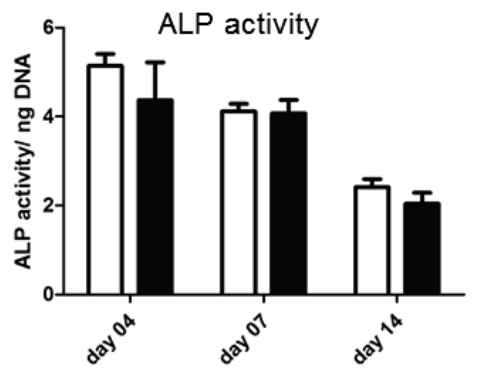

TCP-B

TCP-S

\section{Gene expression}

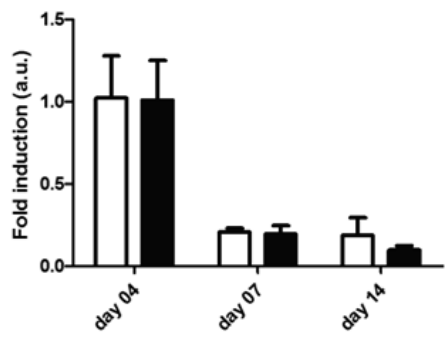

ALP

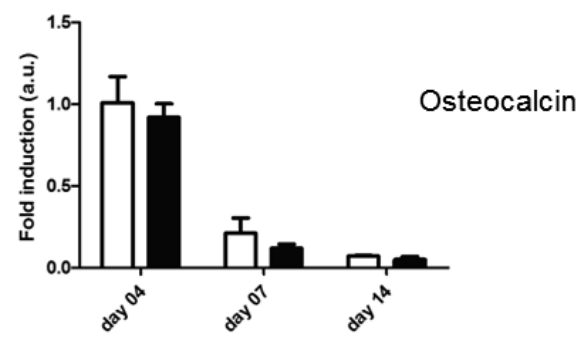

Figure 7. Proliferation (a), ALP activity (b) and gene expression (c) of MG63 cultured on TCP ceramics over time.

A slow proliferation of SAOS-2 cells was seen from day 1 to 7 on both TCP-B and TCP-S, followed by a sharp increase of the cell number from day 7 onward (Figure 8a). A significant higher number of cells was present on TCP-S than on TCP-B at day 14, while such a difference was not present at earlier time points. ALP activity decreased on TCP ceramics over time, but there was no significant difference was observed at all the time points (Figure 
$8 b)$. Gene expression of SAOS-2 was affected by neither the culture time nor the TCP materials (Figure 8c).

a

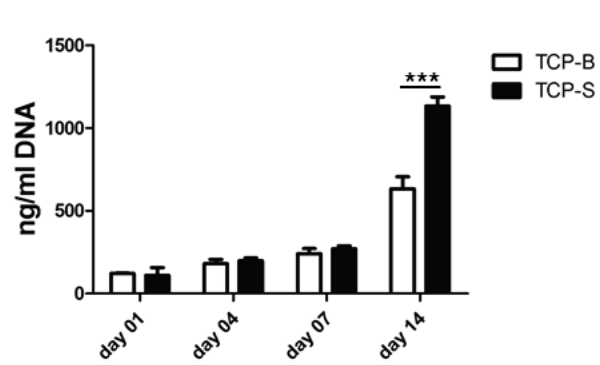

b

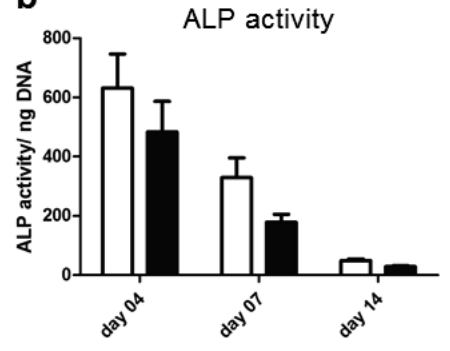

C Gene expression
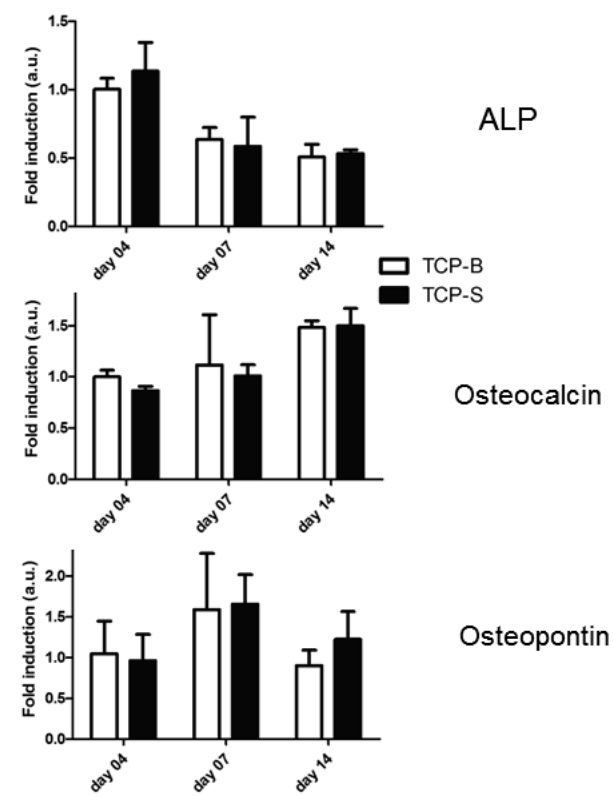

Figure 8. Proliferation (a), ALP activity (b) and gene expression (c) of SAOS-2 cultured on TCP ceramics over time.

\subsection{Discussion}

Small bone defects could be repaired by osteogenic cells present in the host bone bed, while such a conductive bone formation is limited and inductive bone formation is required to repair critical-sized bone defects [3-5, 10]. Several types of bone substitutes are osteoinductive. For example, autologous bone has such property thanks to the growth factors it contains [34]. Introducing osteogenic growth factors (e.g. bone morphogenetic proteins) into biomaterials could make synthetic bone grafting materials osteoinductive [35] and physicochemically modifying synthetics could render biomaterials osteoinductive as well [14]. For instance, adjusting the dimension of surface structure could render CaP ceramics osteoinductive $[16,17]$. 


\section{Chapter 5}

Similarly to other biomaterials, when an osteoinductive CaP ceramic is implanted in vivo, a wound healing process would be initiated by an inflammation, immediately followed by angiogenesis. Later on, bone morphogenesis process would be triggered and eventually bone remodeling occurs. Cells present in the inflammatory bed were found to be sensitive to the surface structure of CaP ceramics. Macrophage grew better and fused into foreign body giant cells on CaP ceramic with submicron scaled surface structure [36]. Monocyte cells grew and fused into osteoclasts on the finer surface structure [18]. Furthermore, the functionality of osteoclasts was affected by the dimension of surface structure: in particular, osteoclasts resorbed TCP ceramic with submicron scaled surface structure [36]. Moreover, CaP ceramics with submicron scaled surface structure enhanced the osteogenic differentiation of human bone marrow MSCs (hBMSCs) [16]. Next to macrophages, monocytes, MSCs and osteoclasts, we have shown in this study that pericytes (HBVP), osteogenically inducible cells (C2C12), osteogenic progenitor cells (MC3T3-E1), pre-osteoblasts (SV-HFO), osteoblasts (MG63) and mature osteoblasts (SAOS-2) responded differently to surface structures with various dimension in terms of cell proliferation, ALP activity and gene expression.

As documented in literature, pericytes may undergo osteogenic differentiation [26, 27, 37]. In absence of growth factor, HBVP gave rise to ALP signal (Figure 1b), but no differences in cell proliferation and osteogenic differentiation were observed between cells on TCP-B and TCP-

S. This indicates that HBVP are not sensitive enough to the surface structure to undergo osteogenic differentiation. The enhanced VEGF gene expression on TCP-B (Figure 2) may imply a possible enhanced angiogenesis in TCP-B implants which, in turn, may bring more pericytes to the implants. However, no inductive bone formation occurred in TCP-B $[16,18$, 36] minimizing further the possible role of pericytes in the enhanced bone formation in osteoinductive CaP ceramics.

BMSCs are quite often used as a stem cell model system. It should be noted that BMSCS readily undergo osteogenic differentiation without any growth factor $[16,38]$ and transplanting bone marrow to ectopic sites gave rise to bone formation [39, 40]. Factors enhancing osteogenic differentiation of BMSCs may thus not be defined as osteoinductive stimuli. Conversely, the osteoinductive function of the surface structure of CaP ceramics may be seen in the result of $\mathrm{C} 2 \mathrm{C} 12$ because these cells do not induce an increase in ALP activity 
unless BMPs are used [41]. Yet, in this study it is interesting to observe that ALP activity of C2C12 cells was enhanced on both ceramics with, in particular, a higher ALP activity on TCP-S (Figure 3b). At the same time, we observed a lower C2C12 cell amount on TCP-S than on TCP-B (Figure 3a), which might indicate that cell proliferation could have been inhibited during their differentiation.

Surface structure has shown its function in bone formation both in vitro and in vivo [42, 43]. For example, at the micron scale the surface structure affected cell proliferation and osteogenic differentiation of osteogenic cells [42]. At the nano-/sub-micro scale, surface structure affected MSCs osteogenic differentiation [44]. Meanwhile, it has been shown that the maturation state of osteogenically committed cells determined their response to the surface structure both in vitro and in vivo [45]. Therefore, we further selected the osteogenic cells tested in this study according to their maturation stage and observed that they behaved differently in vitro. MC3T3-E1 and SAOS-2 cells proliferated slowly over time (Figure 5a, Figure 8a), while a fast cell proliferation at early time points was seen with SV-HFO (Figure 6a) and MG63 (Figure 7a). Regarding osteogenic differentiation, both ALP activity and gene expression of MC3T3-E1 (Figure 5b, 5c) and SV-HFO (Figure 6b, 6c) increased over time, but decreased for MG63 (Figure 7b, 7c) and SAOS-2 (Figure 8b, 8c). Gene expression of osteocalcin was up-regulated in MC3T3-E1 (Figure 5c) and SV-HFO (Figure 6c), downregulated in MG63 (Figure 7c) and did not change in SAOS-2 (Figure 8c) over time. Osteopontin gene expression was down-regulated over time in MC3T3-E1 (Figure 5c), not expressed in MG63 and did not change in SAOS-2 (Figure 8c). In agreement with the literature, different responses to surface structure were also observed within the cells at various maturation stages at the current study settings. On TCP-S, osteoprogenitor cells (e.g. MC3T3-E1) had both enhanced cell proliferation and osteogenic differentiation; preosteoblasts (e.g. SV-HFO) had enhanced osteogenic differentiation; osteblasts (e.g. MG63) and mature osteoblasts (e.g. SAOS-2) had enhanced cell proliferation. The results indicated that immature osteoblasts had both enhanced cell proliferation and osteogenic differentiation on TCP-S as compared to those cultured on TCP-B, while with the maturation of osteoblasts, only cell proliferation could be achieved on TCP-S. 
At the cellular level and in the absence of inductive factors, we have shown in this study the involvement of inducible cells and osteogenic cells at various maturation states in the process of surface structure-driven bone formation. In view of the results shown in this work, the enhanced bone formation within submicron surface-structured osteoinductive $\mathrm{CaP}$ ceramics $[14,16]$ might be attributed to the surface structure-driven osteogenic differentiation of inducible stem cells (both ectopic and orthopedic) and surface structureenhanced conductive bone formation of osteogenic cells in osseous site (orthopedic).

At the genetic level, only osteocalcin was up-regulated in $\mathrm{C} 2 \mathrm{C} 12$ cultured on TCP-S in absence of growth factor. The other genes were either not affected by the surface structure or were up-regulated by TCP-B in all the cells tested. Gene expression data did not support the importance of the surface structure in osteogenesis, while it should be noted that in vivo bone formation is a complicated process and a clear relationship between the gene expressions of ALP, osteocalcin and osteopontin and the in vivo bone formation is still missing at the moment. Furthermore an in vivo implant contacts with body fluids and, consequently, the cells respond not only to the surface structure but also to the soluble cues present in surrounding fluids in vivo (e.g. growth factors, cytokines and ions) [46]. In the presence of soluble cues, the instructive function of surface structure may become more powerful in vivo. For example, in this study we have shown that the presence of rhBMP-2 in the culture medium significantly enhanced osteogenic differentiation (Figure 4b) and upregulated the bone-related gene expressions of ALP, osteocalcin and ostepontin (Figure 4c) of C1C12 cultured on TCP-S.

Together with the literature data, our results show that the surface structure may have a multi-functional role in bone regeneration in vivo with CaP ceramics. It induces macrophages to form foreign body giant cells and monocytes to form osteoclast during the inflammatory stage, influences osteogenic differentiation of inducible cells, increases the proliferation and differentiation of immature osteogenic cells and the proliferation of mature osteogenic cells, and also affects the function of osteoclast for bone remodeling and material resorption.

Biomaterials science is advancing towards instructive materials that can initiate specific biological responses. An example of such instructive materials is the osteoinductive $\mathrm{CaP}$ 
ceramic giving rise to bone formation in ectopic sites and enhancing bone regeneration in orthopedic sites [46]. Some instructive cues of osteoinductive CaP ceramics have been identified, while the biological mechanism of such specific phenomenon is far beyond our understanding. Tissue responses of inflammation [18], osteoclastogenesis [47] and osteogenesis [16] may be involved in such tissue morphogenesis of bone. As shown in this study, cells in different stages of bone formation respond differently to instructive material factors. In vitro studies performed using a single cell type may not be the best way to unravel the biological mechanism of material-driven osteoinduction, systematic studies with reliable in vivo models (e.g. FVB mouse [19]) would be helpful in identifying the cascade of biological phenomena involved in such instructive bone formation.

\subsection{Conclusions}

Cell proliferation and osteogenic differentiation of inducible myoblasts and osteogenic cells at various maturation stages were affected by the surface structure. As compared to micron scaled surface structure, submicron scaled surface structure enhanced osteogenic differentiation of inducible cells and immature osteoblasts while enhanced cell proliferation of mature osteoblasts. The data present herein suggested that both inducible cells and osteogenic cells could respond to surface structure of CaP ceramics and enhanced bone regeneration in submicron surface structured CaP ceramics. 


\section{References}

[1] Bhaskar, S.N., et al., Biodegradable ceramic implants in bone: electron and light microscopic analysis. Oral Surgery, Oral Medicine, Oral Pathology, 1971. 32(2): p. 336-346.

[2] Albee, F.H., Studies in bone growth: triple calcium phosphate as a stimulus to osteogenesis. Annals of surgery, 1920. 71(1): p. 32

[3] Jarcho, M., Calcium phosphate ceramics as hard tissue prosthetics. Clinical orthopaedics and related research, 1981. 157: p. 259-278.

[4] Bohner, M., L. Galea, and N. Doebelin, Calcium phosphate bone graft substitutes: Failures and hopes. Journal of the European Ceramic Society, 2012. 32(11): p. 2663-2671.

[5] Samavedi, S., A.R. Whittington, and A.S. Goldstein, Calcium phosphate ceramics in bone tissue engineering: a review of properties and their influence on cell behavior. Acta Biomaterialia, 2013. 9(9): p. 8037-8045.

[6] Yamasaki, H. and H. Sakai, Osteogenic response to porous hydroxyapatite ceramics under the skin of dogs. Biomaterials, 1992. 13(5): p. 308-312.

[7] Yuan, H., et al., A preliminary study on osteoinduction of two kinds of calcium phosphate ceramics. Biomaterials, 1999. 20(19): p. 1799-1806.

[8] Ripamonti, U., Osteoinduction in porous hydroxyapatite implanted in heterotopic sites of different animal models. Biomaterials, 1996. 17(1): p. 31-35.

[9] Ripamonti, U., J. Crooks, and A. Kirkbride, Sintered porous hydroxyapatites with intrinsic osteoinductive activity: geometric induction of bone formation. 1999.

[10] Habibovic, P., et al., Comparative in vivo study of six hydroxyapatite - based bone graft substitutes. Journal of Orthopaedic Research, 2008. 26(10): p. 1363-1370.

[11] Habibovic, P., et al., 3D microenvironment as essential element for osteoinduction by biomaterials. Biomaterials, 2005. 26(17): p. 3565-3575.

[12] Yuan, H., et al., A comparison of bone formation in biphasic calcium phosphate (BCP) and hydroxyapatite (HA) implanted in muscle and bone of dogs at different time periods. Journal of Biomedical Materials Research Part A, 2006. 78(1): p. 139-147.

[13] Habibovic, P., et al., Relevance of Osteoinductive Biomaterials in Critical - Sized Orthotopic Defect. Journal of Orthopaedic Research, 2006. 24(5): p. 867-876.

[14] Yuan, H., et al., Osteoinductive ceramics as a synthetic alternative to autologous bone grafting. Proceedings of the National Academy of Sciences, 2010. 107(31): p. 13614-13619.

[15] Coathup, M.J., et al., Effect of increased strut porosity of calcium phosphate bone graft substitute biomaterials on osteoinduction. Journal of Biomedical Materials Research Part A, 2012. 100(6): p. 1550-1555.

[16] Zhang, J., et al., The size of surface microstructures as an osteogenic factor in calcium phosphate ceramics. Acta biomaterialia, 2014. 10(7): p. 3254-3263.

[17] Davison, N., et al., Submicron-scale surface architecture of tricalcium phosphate directs osteogenesis in vitro and in vivo. European cells \& materials, 2013. 27: p. 281-297.

[18] Davison, N.L., et al., Liposomal clodronate inhibition of osteoclastogenesis and osteoinduction by submicrostructured beta-tricalcium phosphate. Biomaterials, 2014. 35(19): p. 5088-5097.

[19] Barradas, A.M., et al., The influence of genetic factors on the osteoinductive potential of calcium phosphate ceramics in mice. Biomaterials, 2012. 33(23): p. 5696-5705.

[20] Jiang, Y., et al., Pluripotency of mesenchymal stem cells derived from adult marrow. Nature, 2002. 418(6893): p. 41-49.

[21] Pittenger, M.F., et al., Multilineage potential of adult human mesenchymal stem cells. Science, 1999. 284(5411): p. 143-7.

[22] Colter, D.C., I. Sekiya, and D.J. Prockop, Identification of a subpopulation of rapidly self-renewing and multipotential adult stem cells in colonies of human marrow stromal cells. Proceedings of the National Academy of Sciences, 2001. 98(14): p. 7841-7845.

[23] Song, G., et al., The homing of bone marrow MSCs to non-osseous sites for ectopic bone formation induced by osteoinductive calcium phosphate. Biomaterials, 2013. 


\section{Cells responding to surface structure of calcium phosphate ceramics for bone regeneration}

[24] Bergers, G. and S. Song, The role of pericytes in blood-vessel formation and maintenance. Neuro-oncology, 2005. 7(4): p. 452-464.

[25] Schor, A.M., et al., Pericytes derived from the retinal microvasculature undergo calcification in vitro. Journal of cell science, 1990. 97(3): p. 449-461.

[26] DIAZ-FLORES, L., et al., Pericytes as a supplementary source of osteoblasts in periosteal osteogenesis. Clinical orthopaedics and related research, 1992. 275: p. 280-286.

[27] Doherty, M.J., et al., Vascular pericytes express osteogenic potential in vitro and in vivo. Journal of Bone and Mineral Research, 1998. 13(5): p. 828-838.

[28] Asakura, A., M.A. Rudnicki, and M. Komaki, Muscle satellite cells are multipotential stem cells that exhibit myogenic, osteogenic, and adipogenic differentiation. Differentiation, 2001. 68(4 - 5): p. 245-253.

[29] Kocić, J., et al., Interleukin 17 inhibits myogenic and promotes osteogenic differentiation of C2C12 myoblasts by activating ERK1, 2. Biochimica et Biophysica Acta (BBA)-Molecular Cell Research, 2012.

[30] Chen, S., et al., Synergistic effect of Wnt modulatory small molecules and an osteoinductive ceramic on C2C12 cell osteogenic differentiation. Bone, 2014. 67: p. 109-121.

[31] Boyan, B., et al., Osteoblasts generate an osteogenic microenvironment when grown on surfaces with rough microtopographies. Eur Cell Mater, 2003. 6(24): p. 22-27.

[32] Chiba, H., et al., Establishment and Characterization of a Simian Virus 40 - Immortalized Osteoblastic Cell Line from Normal Human Bone. Cancer Science, 1993. 84(3): p. 290-297.

[33] Van Driel, M., et al., Evidence that both $1 \alpha, 25$ - dihydroxyvitamin D3 and 24 - hydroxylated D3 enhance human osteoblast differentiation and mineralization. Journal of cellular biochemistry, 2006. 99(3): p. 922-935.

[34] Finkemeier, C.G., Bone-grafting and bone-graft substitutes. The Journal of Bone \& Joint Surgery, 2002. 84(3): p. 454-464.

[35] Edlund, U., S. Dånmark, and A.-C. Albertsson, A strategy for the covalent functionalization of resorbable polymers with heparin and osteoinductive growth factor. Biomacromolecules, 2008. 9(3): p. 901-905.

[36] Davison, N.L., et al., Osteoclast resorption of beta-tricalcium phosphate controlled by surface architecture. Biomaterials, 2014. 35(26): p. 7441-7451.

[37] Diaz - Flores, L., et al., Inducible perivascular cells contribute to the neochondrogenesis in grafted perichondrium. The Anatomical record, 1991. 229(1): p. 1-8.

[38] Prins, H.-J., et al., In vitro induction of alkaline phosphatase levels predicts in vivo bone forming capacity of human bone marrow stromal cells. Stem Cell Research, 2014. 12(2): p. 428-440.

[39] Friedenstein, A.J., et al., HETEROTOPIC TRANSPLANTS OF BONE MARROW. Transplantation, 1968. 6(2): p. 230-247.

[40] Friedenstein, A., I. Piatetzky-Shapiro, and K. Petrakova, Osteogenesis in transplants of bone marrow cells. Journal of embryology and experimental morphology, 1966. 16(3): p. 381-390.

[41] Katagiri, T., et al., Bone morphogenetic protein-2 converts the differentiation pathway of C2C12 myoblasts into the osteoblast lineage. The Journal of cell biology, 1994. 127(6): p. 1755-1766.

[42] Schwartz, Z., et al., Implant surface characteristics modulate differentiation behavior of cells in the osteoblastic lineage. Advances in Dental Research, 1999. 13(1): p. 38-48.

[43] Olivares - Navarrete, R., et al., Osteoblast maturation and new bone formation in response to titanium implant surface features are reduced with age. Journal of Bone and Mineral Research, 2012. 27(8): p. 1773-1783.

[44] Oh, S., et al., Stem cell fate dictated solely by altered nanotube dimension. Proceedings of the National Academy of Sciences, 2009. 106(7): p. 2130.

[45] Lohmann, C., et al., Maturation State Determines the Response of Osteogenic Cells to Surface Roughness and 1, 25 - Dihydroxyvitamin D3. Journal of Bone and Mineral Research, 2000. 15(6): p. 1169-1180.

[46] Barradas, A.M., et al., A calcium-induced signaling cascade leading to osteogenic differentiation of human bone marrow-derived mesenchymal stromal cells. Biomaterials, 2012. 33(11): p. 3205-15.

[47] Nasu, T., et al., EP4 agonist accelerates osteoinduction and degradation of $\beta$-tricalcium phosphate by stimulating osteoclastogenesis. Journal of Biomedical Materials Research Part A, 2009. 89(3): p. 601-608. 



\section{Chapter 6}

\section{Microporous calcium phosphate ceramics driving osteogenesis through surface architecture}

Jingwei Zhang ${ }^{a, b, \S}$, Davide Barbieri ${ }^{c, \S}$, Hetty ten Hoopen ${ }^{d}$, Joost D. de Bruijn ${ }^{b, e, f}$, Clemens A. van Blitterswijk ${ }^{\mathrm{a}, \mathrm{g}}$, Huipin Yuan ${ }^{\mathrm{a}, \mathrm{c}, \mathrm{g}, \mathrm{h}, *}$

a Department of Tissue Regeneration, MIRA Institute for Biomedical Technology and Technical Medicine, University of Twente, P.O. Box 217, 7500AE Enschede, The Netherlands

${ }^{b}$ Key Laboratory of Advanced Technologies of Materials, Ministry of Education, School of Materials Science and Engineering, Southwest Jiaotong University, Chengdu, P.R. China

${ }^{c}$ Xpand Biotechnology BV, Bilthoven, The Netherlands

${ }^{d}$ Biomedical Chemistry, MIRA Institute for Biomedical Technology and Technical Medicine, University of Twente, P.O. Box 217, 7500AE Enschede, The Netherlands

e Department of Biomaterials Science and Technology, MIRA Institute for Biomedical Technology and Technical Medicine, University of Twente, P.O. Box 217, 7500AE Enschede, The Netherlands

${ }^{f}$ School of Engineering and Materials Science(SEMS), Queen Mary University of London, E1 4NS London, United Kingdom

${ }^{g}$ College of Physical Science and Technology, Sichuan University, Chengdu, China

${ }^{\S}$ Contributed equally to this work 


\section{Chapter 6}

\section{Abstract}

The presence of micropores in calcium phosphate $(\mathrm{CaP})$ ceramics has shown its important role in initiating inductive bone formation in ectopic sites. To investigate how microporous calcium phosphate ceramics trigger osteoinduction, we optimized two biphasic calcium phosphate ceramics (i.e. BCP-R and BCP-S) to have the same chemical composition, equivalent surface area per volume, comparable protein adsorption, similar ion (i.e. calcium and phosphate) exchange and the same surface mineralization potential, but different surface architecture. In particular, BCP-R had a surface roughness (Ra) of $325.4 \pm 58.9 \mathrm{~nm}$ while for BCP-S it was $231.6 \pm 35.7 \mathrm{~nm}$. Ceramic blocks with crossing or non-crossing channels of 250, 500, 1000 and $2000 \mu \mathrm{m}$ were implanted in paraspinal muscle of dogs for 12 weeks. The percentage of bone volume in the channels was not affected by the type of pores (i.e. crossing vs. closed) or their size, but it was greatly influenced by the ceramic type (i.e. BCP-R vs. BCP-S). Significantly more bone was formed in the channels of BCP-R than in those of BCP-S. Since the two CaP ceramics differed only in their surface architecture, the results hereby demonstrate that microporous $\mathrm{CaP}$ ceramics may induce ectopic osteogenesis through surface architecture.

\section{Keywords:}

Bone grafting, Calcium phosphate ceramics, Microstructure, Surface architecture, Osteogenesis. 


\subsection{Introduction}

Searching for alternatives to autologous bone graft, which is the current gold standard grafting material to heal bone defects, has become a goal of biomedical engineering [1-3]. Calcium phosphate ( $\mathrm{CaP}$ ) ceramics are applied for bone grafting because their chemical composition resembles the bone mineral phase [4-6]. Traditionally, CaP ceramics were used as osteoconductive or osteoinductive bone graft substitutes in combination with cells and/or growth factors. For instance, CaP ceramics delivering mesenchymal stromal cells (MSCs) to the bone defect site could induce bone formation [7]. Alternatively, a subclass of CaP ceramics with specific physicochemical properties can induce ectopic bone formation without the addition of cells or growth factors through a process defined as "osteoinduction". Such phenomena has been reported in several animal models, where hydroxyapatite (HA) ceramic induced heterotopic bone formation in dogs $[8,9]$, baboons [4, 10-12], rabbits [13], mouse [13], sheep [14] and goats [15, 16]. Similarly, it was shown occurring also in tri-calcium phosphate (TCP) ceramics in dogs [4, 9, 17-20], sheep [21], and in biphasic calcium phosphate (BCP) ceramics implanted in dogs [5, 17, 18, 22], pigs [5], sheep [14, 23], goat [16, 24-27], rabbits [13, 28] and baboons [11]. Previous studies have shown the possible clinical importance of such CaP ceramics by comparing them in both non-osseous and osseous sites [22, 29]. Moreover, a resorbable osteoinductive TCP ceramic has shown equivalent bone forming ability to autologous bone graft and recombinant human bone morphogenetic protein-2 (rhBMP-2) in critical-sized bone defects [21]. This finding strongly reveals the potential of such specific ceramics to be alternatives to autologous bone grafts.

The available data may have shown the relevance of material properties to its functionality in bone regeneration. Physicochemical modification of CaP ceramics may represent an approach for bone defect repair. It is well known that the chemistry of CaP ceramics influences on their degradation behavior, which in turn affects osteogenesis. For instance, in a previous study of ours, HA and BCP ceramics were implanted intramuscularly in goats: higher bone incidence and amount were found in the BCP ceramic - which contains the more soluble TCP - as compared to HA ceramic [15]. The geometry and macrostructural properties 


\section{Chapter 6}

of ceramic scaffolds have been shown to play critical roles in bone formation as well. Ripamonti et al. showed that bone formation always started from the concave side of the macropores and never from the convex spaces of HA rods and discs [30].

It is generally accepted that the presence of micropores in CaP ceramics plays an important role in inducing heterotopic bone formation. For instance, microporous HA ceramics could induce bone formation after subcutaneous [8] and intramuscular [6] implantations in dogs, while no bone formed in those HA ceramics lacking of micropores. The microstructure may improve osteoinduction by enlarging the surface area, thus affecting protein adsorption, ion release and mineral deposition. Protein adsorption, i.e. the adsorption of growth factors including bone morphogenetic proteins (BMPs) [31, 32], is believed to play a crucial role in bone induction. It has therefore been suggested that micropores in CaP ceramics may concentrate higher amounts of growth factors thanks to their enlarged surface area [15, 21]. It was also proposed that the microporous CaP ceramics can enhance the ion exchange processes because of their enlarged surface area, later affecting osteogenic differentiation of MSCs [33]. The ions (e.g. $\mathrm{Ca}^{2+}$ and $\mathrm{PO}_{4}{ }^{3-}$ ) in the surroundings might trigger the bioactivity of CaP ceramics, which may later allow bone formation [34]. In addition, the Ca and P ions in the body fluids could also form a biological apatite layer on the surface of the CaP ceramics $[16,35]$. The possible role of the formation of an apatite layer in osteoinduction is supported by the fact that bioactive metals could induce ectopic bone formation. For example, bioactive titanium implants that could get surface mineralization induced bone formation after ectopic implantation, while titanium implants not able to get such mineralization then could not induce any bone formation [35].

In addition to the improved protein adsorption and ion exchange, micropores in $\mathrm{CaP}$ ceramics can generate different surface architectures, which have shown to influence the osteogenic differentiation of MSCs through various physical properties such as surface roughness [36-40], the size of surface pattern [41, 42] and surface morphology [43-45]. For instance, Fu et al. showed that micropost rigidity can affect cell morphology, focal adhesions, cytoskeletal contractility and differentiation of stem cells [46]. In addition, Oh et al. showed the critical role of the nanotube dimensions on osteogenic differentiation of hMSCs in absence of osteogenic inducing media [42]. Finally, Dalby et al. demonstrated that nanoscale 
disorder could stimulate osteogenic differentiation of hMSCs in vitro without osteogenic supplements [45]. Therefore the role of surface architecture of CaP ceramics in ectopic osteogenesis cannot be excluded. The surface arthitecture could be varied by controlling the sintering temperature of CaP ceramics: a decrease in grain size and increase in microporosity have shown to positively affect on osteoinductive potential of CaP ceramics [16, 21]. Considering the surface properties, it is known that the presence of a high number of small features on the surface indicates high roughness values.

Therefore, from previous results suggesting that $\mathrm{CaP}$ ceramics with small grains trigger heterotopic bone formation, here we expect that CaP ceramics with high roughness will lead to ectopic bone formation. To explore whether the surface architecture affects osteogenesis by microstructured CaP ceramics, we optimized two types of BCP ceramics (i.e. BCP-R and BCP-S) to have the same chemical composition, equivalent surface area per volume, comparable protein adsorption, similar ions (e.g. $\mathrm{Ca}^{2+}$ and $\mathrm{PO}_{4}{ }^{3-}$ ) exchange and same surface mineralization potential, but different surface architecture. We then compared their capacity to induce osteogenesis in a canine osteoinduction model.

\subsection{Materials and methods}

\subsubsection{Preparation and optimization of BCP ceramics}

Two types of BCP, namely BCP-R and BCP-S, were prepared from the same CaP apatite powder with a $\mathrm{Ca} / \mathrm{P}$ ratio of 1.64 . BCP-R was obtained by mixing $\mathrm{CaP}$ apatite powder with $0.1 \%$ $\mathrm{H}_{2} \mathrm{O}_{2}$ solution to get a slurry, which was subsequently dried at room temperature and sintered at $1150^{\circ} \mathrm{C}$ for 8 hours. BCP-S was prepared by isostatically pressing the starting apatite powder in a steel cylinder, with inner diameter of 1.25 inch, at a certain pressure for 5 minutes at room temperature and thereafter sintering it at $1150^{\circ} \mathrm{C}$ for 8 hours. Importantly, the pressure was optimized to get BCP-S having the same protein adsorption as $\mathrm{BCP}-\mathrm{R}$ (see §2.3): at last the chosen BCP-S was prepared using a pressure of $50 \mathrm{MPa}$. The ceramic samples were then made into three forms. Discs $(\varnothing 10 \times 1 \mathrm{~mm})$ were fabricated using a lathe (Esmeijer, Netherland) and a histological diamond saw (SP-1600, Leica, Germany), while blocks $(7 \times 9 \times 12 \mathrm{~mm})$ were made using a diamond saw (Heathway, England). Afterwards, two types of channels (i.e. crossing and non-crossing) with different diameters 


\section{Chapter 6}

of 250, 500, 1000 and $2000 \mu \mathrm{m}$ were made along the shortest side of the BCP blocks using drills with the required diameter. BCP-R ceramic implants had both crossing and noncrossing channels, while BCP-S ceramic implants only had non-crossing channels. Due to technical challenges, it was not possible to obtain crossing channels with a diameter of 250 $\mu \mathrm{m}$ in BCP-R. Granules (size 1-2 $\mathrm{mm}$ ) were obtained by crushing and sieving the ceramics. The crossing channels were made to study the possible role of pore interconnectivity in bone induction. All the samples were ultrasonically cleaned with acetone, $70 \%$ ethanol and demineralized water, then dried at $80^{\circ} \mathrm{C}$ and sterilized with gamma irradiation (Isotron, Netherland BV, Ede, The Netherlands) before further use.

\subsubsection{Physicochemical characterization of the ceramics}

The chemical composition of the samples was determined with X-ray diffraction (XRD; Rigaku, Japan). The HA/ $\beta$-TCP weight ratios in the BCP ceramics were determined with a calibration line obtained from internal standard powders with known HA/ $\beta$-TCP weight ratios. The surface microstructure of the BCP ceramics was studied with scanning electron microscope (SEM; XL30, ESEM-FEG, Philips, Eindhoven, The Netherlands) in the secondary electron mode. The micro-porosity, micro-pore size distribution and specific surface area of the ceramic blocks were measured using a mercury intrusion porosimeter (MP; Auto Pore IV 9500, Micromeritics European Analysis Service, Monchengladbach, Germany). The density of the ceramics was measured with the weight/volume method using the ceramic discs. To better evaluate the surface properties, we calculated the surface area per volume as the product between the specific surface area measured with mercury intrusion and the measured density of the ceramic. The surface roughness of the ceramic discs was analyzed with atomic force microscopy (AFM; Bioscope Catalyst AFM, Bruker AXS, Wormer, the Netherlands) using dual scans in tapping mode, at a scan frequency of $1 \mathrm{~Hz}$, on areas of $10 \times 10 \mu \mathrm{m}$ with a MikroMarsch NCS36 cantilever (total tip height 20-25 $\mu \mathrm{m}$, tip radius $10 \mathrm{~nm}$ and full type cone radius of $40^{\circ}$, spring constant $1.75 \mathrm{~N} / \mathrm{m}$ ). Obtained images were filtered from signal noise and then analyzed with SPIP software (v5.1.11, Image Metrology A/S, Hørsholm, Denmark). The surface roughness was estimated in terms of roughness average (Ra) and root mean square ( $\mathrm{Rq})$. Further, measurements on the profile height changes compared to the zero-plan were also taken and plotted as distribution. 


\subsubsection{Protein adsorption}

Fetal bovine serum (FBS, Lonza, Germany) and bovine serum albumin (BSA, Sigma) were diluted in $25 \mathrm{ppm}$ aqueous $\mathrm{NaN}_{3}$ solution and sterilized with $0.22 \mu \mathrm{m}$ filter to get the solutions of FBS $(0.25 \%)$ and BSA $(100 \mu \mathrm{g} / \mathrm{ml})$. After immersing the discs in the solutions (3 $\mathrm{ml}$ per disc, $\mathrm{n}=5$ per material) at $37^{\circ} \mathrm{C}$ for 24 hours, the remaining proteins in the solutions were measured with the QuantiPro ${ }^{T M}$ BCA assay kit (Pierce, The Netherlands) following the manufacturer's guidelines and absorbance was measured with a spectrophotometer (AnthosZenyth 3100, AnthosLabtec Instruments GmbH, Salzburg, Austria) at $562 \mathrm{~nm}$. The amount of proteins adsorbed from FBS solution onto the discs ( $\mu \mathrm{g}$ of protein per disc) was estimated through a calibration serum protein curve using FBS after determining the total proteins content in pure FBS (i.e. $33.78 \pm 0.64 \mathrm{mg} / \mathrm{ml}$ ). In the meantime, a calibration line for BSA was built with a series of concentrated BSA solutions. All measured values were expressed as mean \pm standard deviation.

\subsection{4. rhBMP-2 adsorption}

A solution of rhBMP2 (500 ng/ml) was prepared by diluting rhBMP-2 (Reborne, Shanghai) in the basic medium (BM) containing Dulbecco's modified Eagle's medium (DMEM; Gibco, Invitrogen, UK), 10\% FBS and 1\% penicillin/streptomycin $(100 \mathrm{U} / \mathrm{ml}$ penicillin, $100 \mathrm{mg} / \mathrm{ml}$ streptomycin; Gibco, Invitrogen, UK). The ceramic discs were then soaked in the medium (3 $\mathrm{ml}$ per disc, $\mathrm{n}=5$ per material) at $37^{\circ} \mathrm{C}$ in humid atmosphere with $5 \% \mathrm{CO}_{2}$ for 24 hours. The discs were stored at $-80^{\circ} \mathrm{C}$ for at least 12 hours after being washed with PBS three times. Thereafter $0.5 \mathrm{ml}$ of $1 \%$ triton was add to each sample and kept at $4{ }^{\circ} \mathrm{C}$ for at least 12 hours and followed by ultrasonically shaking for 5 minutes. Finally, rhBMP-2 content in the supernatant was measured with BMP-2 Elisa kit (R\&D, UK) following the manufacturer's instructions and absorbance was measured with a spectrophotometer at $450 \mathrm{~nm}$. The rhBMP-2 adsorption was estimated through a standard calibration rhBMP-2 curve and expressed as mean \pm standard deviation.

\subsubsection{Ion exchange}

Ceramic discs were soaked in $\mathrm{BM}\left(3 \mathrm{ml}\right.$ per disc, $\mathrm{n}=5$ per material) at $37^{\circ} \mathrm{C}$ in humid atmosphere with $5 \% \mathrm{CO}_{2}$ for 24 hours. $\mathrm{Ca}$ and $\mathrm{P}$ contents in the solutions were measured 


\section{Chapter 6}

and compared to the control (i.e. solution with no sample). Ca and P ion concentration were measured with QuantiChrom ${ }^{\mathrm{TM}} \mathrm{Ca}$ assay kit (BioAssay, USA) and phosphoWork ${ }^{\mathrm{TM}}$ Colorimetric P Assay Kit (AATBioquest, USA) respectively following the manufacturer's guidelines. Thereafter, absorbance was measured with spectrophotometer at $620 \mathrm{~nm}$. Both $\mathrm{Ca}$ and $\mathrm{P}$ contents were expressed as mean \pm standard deviation.

\subsubsection{Surface mineralization}

Simulated body fluid (SBF) was prepared according to literature [47] by dissolving reagent grade chemicals in distilled water strictly in the following order: $\mathrm{NaCl}, \mathrm{NaHCO}_{3}, \mathrm{KCl}$, $\mathrm{K}_{2} \mathrm{HPO}_{4} \cdot 3 \mathrm{H}_{2} \mathrm{O}, \mathrm{MgCl}_{2} \cdot 6 \mathrm{H}_{2} \mathrm{O}, \mathrm{CaCl}_{2}$ (Ca ion standard solution, $0.1 \mathrm{M}$, Metrohm) and $\mathrm{Na}_{2} \mathrm{SO}_{4}$. The solution was then buffered to $\mathrm{pH} 7.4$ at $36.5^{\circ} \mathrm{C}$ using Tris $\left(\left(\mathrm{CH}_{2} \mathrm{OH}\right)_{3} \mathrm{CNH}_{3}\right)$ and $1 \mathrm{M} \mathrm{HCl}$. The final solution had an ion concentration (mM) as follows: $\mathrm{Na}^{+}, 142 ; \mathrm{K}^{+}, 5 ; \mathrm{Mg}^{2+}, 1.5 ; \mathrm{Ca}^{2+}$, $2.5 ; \mathrm{Cl}^{-}, 147.8 ;\left(\mathrm{HCO}_{3}\right)^{-}, 4.2 ;\left(\mathrm{HPO}_{4}\right)^{2-}, 1 ;\left(\mathrm{SO}_{4}\right)^{2-}, 0.5$. Fifty granules $(1-2 \mathrm{~mm})$ of each material were soaked in $200 \mathrm{ml}$ of SBF at $37 \pm 1^{\circ} \mathrm{C}$ for up to 1 week. A minimum of 10 granules were taken out at each time point of 1, 2, 4 and 7 days. They were rinsed five times with distilled water and dried at room temperature. The mineralization of the BCP ceramics was observed with SEM. Four spots were randomly selected from each granule. The mineralization level of the materials was ranked as follows: (a) no mineralization: all the spots were completely bare; (b) few mineralization: one (or more) of the spots had thin and not uniform mineralized layers; and (c) full mineralization: all the spots were covered by thick and uniform mineralized layers. For each classification group, the number of granules was counted and plotted on a histogram as percentage on the total granules considered.

\subsubsection{Evaluation of osteoinduction}

\subsubsection{Animal experiments}

Following the permission of the local animal care committee (Animal Center, Sichuan University, Chengdu, China), ceramic blocks ( $n=8$ per ceramic) were implanted in the paraspinal muscles of 8 adult male dogs (mongrel, 10-15 kg) for 12 weeks. The surgical operation was performed under general anaesthesia by abdominal injection of sodium pentobarbital (30 mg/kg body weight) and sterile conditions. Following the surgeries, buprenorphine (0.1 mg per animal) was intramuscular injected to the dogs for 2 days to relieve pain, while 
penicillin $(40 \mathrm{mg} / \mathrm{kg}$ ) was intramuscular injected for three consecutive days to prevent infection. After operation, the animals were allowed for full weight bearing and received normal diet.

\subsubsection{Histological processing}

After 12 weeks the animals were sacrificed with a celiac injection of excessive amount of pentobarbital sodium. Implants were then harvested with the surrounding tissues and fixed in $4 \%$ buffered formaldehyde solution $(\mathrm{PH}=7.4)$ at $4^{\circ} \mathrm{C}$ for one week, and embedded in methyl methacrylate (MMA, K-plast, LTI Netherland, Bilthoven, the Netherlands) after dehydration in a series of gradient ethanol solutions. Serial non-decalcified sections with an average thickness of $15 \mu \mathrm{m}$ were obtained without interruption using a microtome (SP-1600, Leica, Germany) equipped with diamond saw blade having an approximate thickness of 280 $\mu \mathrm{m}$ and a knurled screw to control the thickness of the sections. Being the sectioning done serially, the distance between consecutive sections is approximately $280 \mu \mathrm{m}$ due to loss of material by the blade. Each slide was stained with $1 \%$ methylene blue (Aldrich) and $0.3 \%$ basic function (Aldrich). Sections were also prepared from MMA-embedded ceramic blocks (not implanted) to be used as controls.

\subsubsection{Histological and histomorphometric analysis}

The histological sections were observed with light microscopy (Nikon Eclipse E200, Tokyo, Japan) with respect to tissue formation in the channels. The sections were then scanned using a slide scanner (Dimage Scan Elite 5400II, Konica Minolta Photo Imaging Inc, Tokyo, Japan) to obtain overview images for histomorphometrical analysis, which was performed using Adobe Photoshop Elements 4.0 software. The channel area was selected as a region of interest and the corresponding number of pixels was read as ROI. Then the bone tissue area was pseudo-colored and its respective pixels were counted as B. The percentage of bone area in the channel (B\%) was determined as $\mathrm{B} \%=\mathrm{B} * 100 / \mathrm{ROI}$ and the results were presented as mean and standard deviation of all the sections taken at the same depth from all the samples. As shown in the 

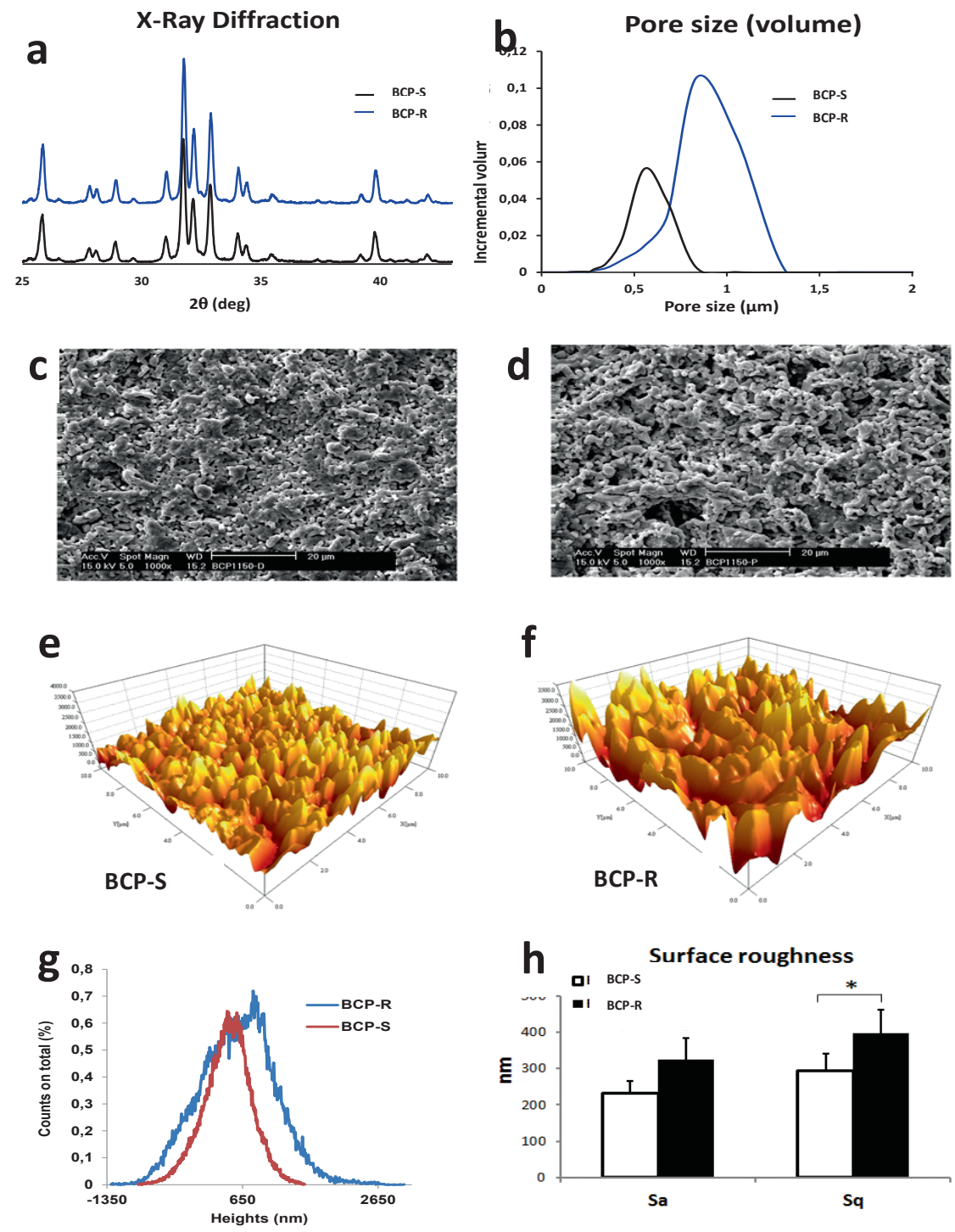

Figure 1. (a) XRD pattern of the two ceramics, showing that BCP-S and BCP-R have the same chemistry. (b) Micropore distribution of BCP-S and BCP-R as obtained from mercury intrusion measurements. It can be seen that BCP-S has smaller micropore size than BCP-R. The surface microstructure of (c) BCP-S and (d) BCP-R was observed with SEM. AFM allows evaluating the surface roughness of (e) BCP-S and $(f) B C P-R$. The height distributions of the microstructured surface of the two ceramics is shown in (g). Histograms in (h) allow the comparison of the measured surface roughness average (Sa) and root mean square (Sq) between the two ceramics. Statistically significant differences are marked by * for $p<0.05$. 
equation mentioned above, we normalized $\mathrm{Bp} \%=\frac{\mathrm{B}^{*} 100}{\mathrm{ROI}-\mathrm{M}}$ the bone area to the ROI of the corresponding channel to make the comparison of bone formation among the channels with different dimension possible. The ROI of the channels in the control ceramic blocks was measured as well, and the resorption rate (R\%) of the ceramics after explant was determined as $\mathrm{R} \%=(\mathrm{ROI}$ of the control-ROI of the explants)*100/(ROI of the control). The measurements were taken both at the openings and in the central region of the channels.

\subsubsection{Statistical Analysis}

Statistical analysis was performed using a one-way ANOVA followed by a Tukey's multiple comparison test or paired t test. A value of $p<0.05$ was considered as significant difference.

Table 1. Density, specific surface area and surface area per volume of the two BCP ceramics: the density and the specific surface area data were experimentally measured and used to calculate the surface area per volume that resulted to be the same for the two ceramics.

\begin{tabular}{cccc}
\hline & $\begin{array}{c}\text { Density } \\
(\mathrm{g} / \mathrm{mL})\end{array}$ & $\begin{array}{c}\text { Specific Surface area } \\
\left(\mathrm{m}^{2} / \mathrm{g}\right)\end{array}$ & $\begin{array}{c}\text { Surface area per volume } \\
\left(\mathrm{m}^{2} / \mathrm{mL}\right)\end{array}$ \\
\hline BCP-R & $1.758 \pm 0.026$ & 1.057 & 1.859 \\
BCP-S & $2.242 \pm 0.026$ & 0.825 & 1.854 \\
\hline
\end{tabular}

\subsection{Results}

\subsubsection{Material characteristics}

The XRD patterns of the ceramics are given in Figure 1a, which shows the two BCP ceramics had similar chemistry consisting of $80 \pm 3 w t \%$ HA and $20 \pm 3 w t \% \beta$-TCP. The SEM observation demonstrated a rougher surface of BCP-R (Figure 1d) than that of BCP-S (Figure 1c). This observation was further confirmed by AFM images (Figures 1e and 1f). Smaller pores could also be observed on BCP-S than BCP-R as confirmed also by mercury intrusion test (Figure 1b). The average roughness ( $R a)$ and the root mean square ( $R q)$ resulted significantly higher in BCP-R than BCP-S ( $p=0.0014$ and $p=0.0019$ respectively) indicating that the former material presents a topography having larger and deeper variability in its peaks and hollows heights over the area (Figure $1 \mathrm{~h}$ ). These results are corroborated by the distribution of 


\section{Chapter 6}

surface heights (Figure 1g), which is larger for BCP-R. Interestingly, the distribution of surface heights on BCP-R presents a lager tail on the right as compared to BCP-S, which might be correlated with the preparation method of BCP-S involving a step of isostatic pressure that could have flattened the surface. In addition, the mercury intrusion results showed the two $\mathrm{BCP}$ ceramics had different porosity and distribution of micropore size. A micropore range of 0.3 and $1.3 \mu \mathrm{m}$ with a peak at $0.9 \mu \mathrm{m}$ for BCP-R, whereas BCP-S had micropore range from 0.3 to $0.7 \mu \mathrm{m}$ with a peak at $0.6 \mu \mathrm{m}$ (Figure $1 \mathrm{~b}$ ). The total microporosity was higher for BCP$\mathrm{R}(43 \%)$ than for BCP-S (28\%). Mercury intrusion data revealed also that the surface area per volume of BCP-R and BCP-S were the same (Table 1).

\subsubsection{Protein adsorption assay}

The amount of protein absorbed by the two ceramics is shown in Figures $2 c, 2 d$ and $2 e$, where no significant differences between BCP-R and BCP-S with respect to adsorption of BSA $(P=0.702)$, of proteins in FBS $(P=0.387)$ and of rhBMP-2 $(P=0.632)$ were observed. These results might be explained by the fact that the two ceramics have the same surface area.

\subsection{3. $\mathrm{Ca}$ and $\mathrm{P}$ ions assay}

Changes in $\mathrm{Ca}$ and $\mathrm{P}$ ion concentrations in $\mathrm{BM}$ were evaluated (Figures $2 \mathrm{a}$ and $2 \mathrm{~b}$ ): instead of releasing $\mathrm{Ca}$ and $\mathrm{P}$ ions into the medium, both ceramics adsorbed ions from the medium within 24 hours. However, BCP-R and BCP-S adsorbed similar amounts of $\mathrm{Ca}$ and $\mathrm{P}$ ions and no significant differences were observed (Ca: $p=0.153, P: p=0.088)$.

\subsubsection{Surface mineralization}

Mineralization on the BCP ceramics was observed as early as after soaking in SBF for one day (Figure $3 a$ and $3 b$ ), and the amount of mineralization increased during time finally leading to thick layers of apatite that uniformly covered the surface of all the samples after seven days (Figure $3 c$ and $3 d$ ). The trend of surface mineralization over time is illustrated in Figure $3 e$, where no significant differences between the two BCP ceramics were observed after seven days. 

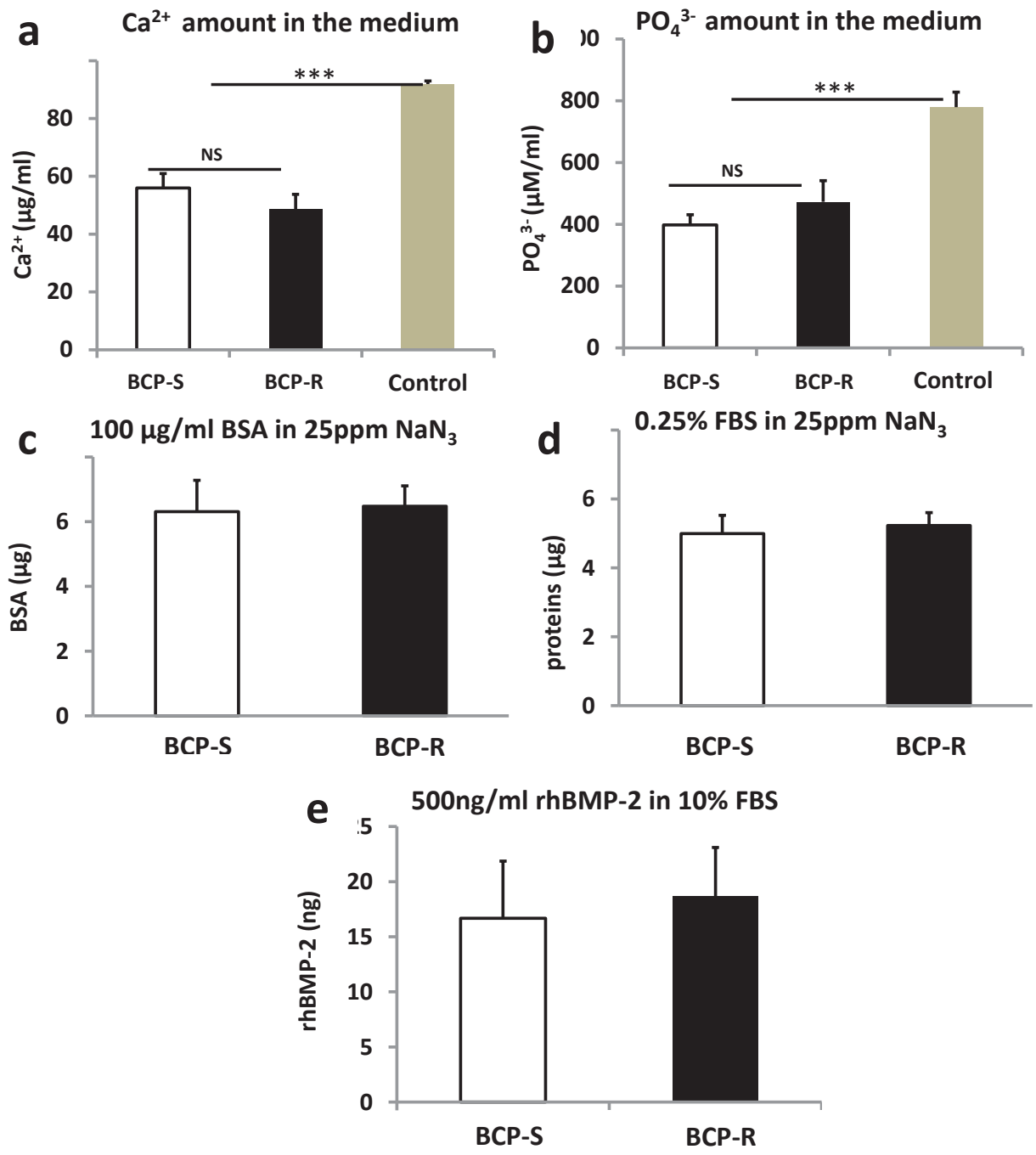

Figure 2. (a) $\mathrm{Ca}^{2+}$ and (b) $\mathrm{PO}_{4}{ }^{3-}$ concentrations in culture medium after soaking the two ceramics' discs for 24 hours: they were significantly lower than the control medium in both cases, but differences in $\mathrm{Ca}^{2+}$ and $\mathrm{PO}_{4}{ }^{3-}$ amounts in the medium with $B C P-S$ and $B C P-R$ were not significant. Protein adsorption of the ceramic discs after soaking in (c) BSA, (d) FBS and (e) rhBMP-2 solutions, indicating that BCP-S and BCP-R have similar protein adsorption potential. Any statistically significant difference is marked with ${ }^{* * *}$ for $p<0.001$. 


\section{Chapter 6}

a

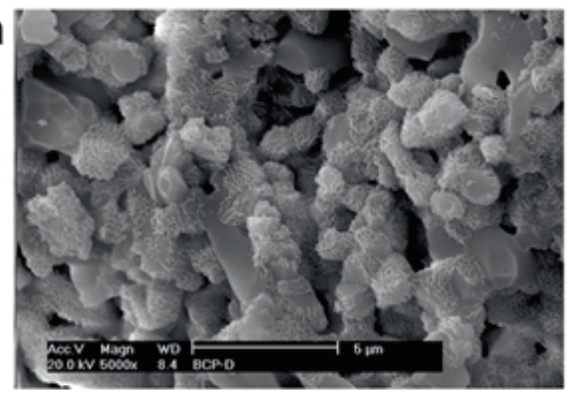

C
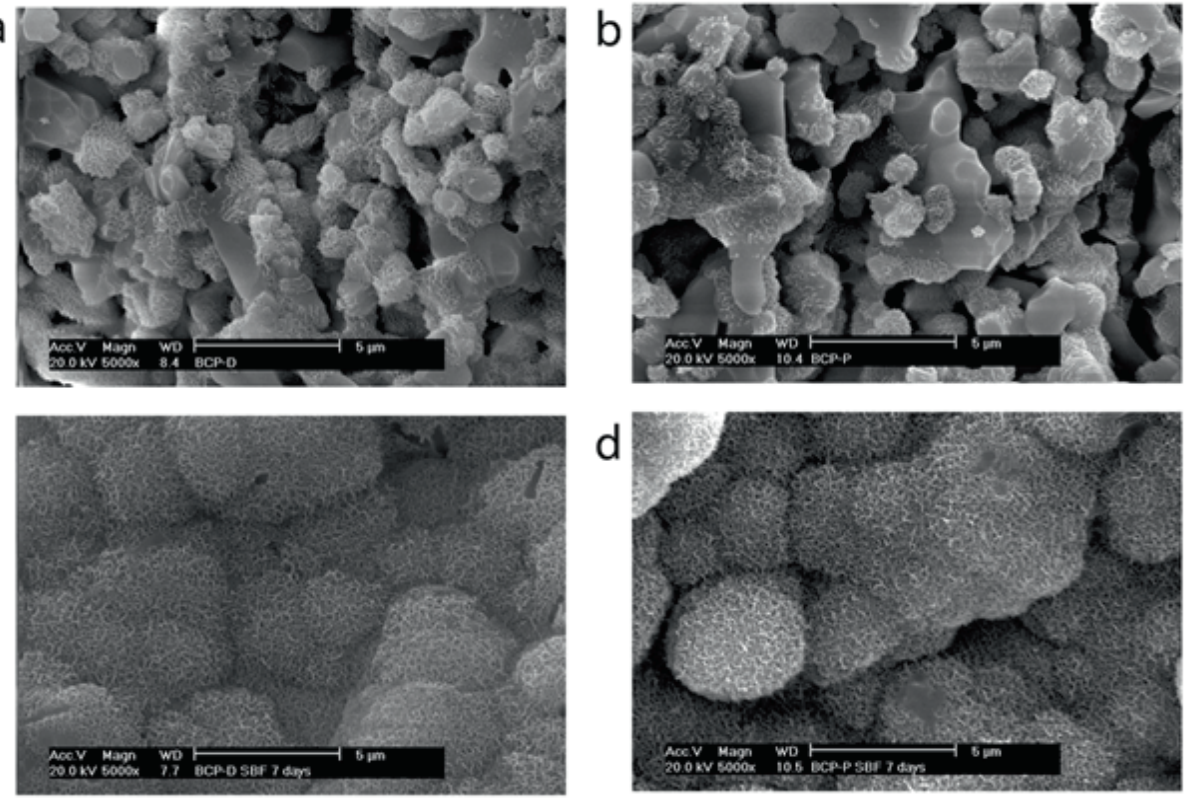

d

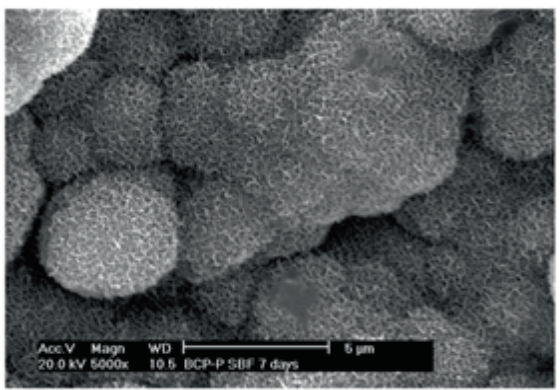

e

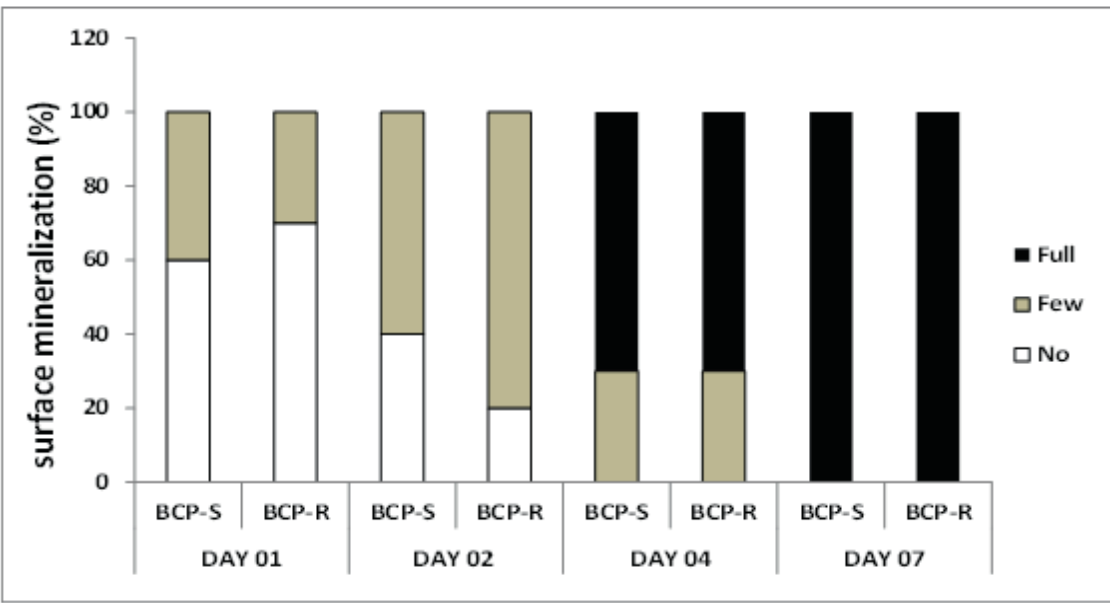

Figure 3. Surface mineralization of BCPs after soaking in SBF solution. SEM images of surface mineralization on BCP-S with (a) few and (c) full surface mineralization and BCP-R with (b) few and (d) full surface mineralization. (e) Percentage of BCPs ceramics with no, few and full surface mineralization at days 1, 2, 4 and 7. 


\subsubsection{Ectopic bone formation}

A total of eight samples per ceramic were intramuscularly implanted in eight dogs for 12 weeks, and all the samples were retrieved. Histological observations demonstrated that bone formed in most channels of BCP-R (Figure $4 \mathrm{~b}$ and $4 \mathrm{~d}$ ), while in BCP-S little bone amounts were found in few channels and fibrous tissue was dominant (Figure 4a and 4c). Bone grew tight to the wall of the channels (Figure 4d). The distribution of the newly formed bone along the crossing channels with different diameters (i.e. $500 \mu \mathrm{m}, 1000 \mu \mathrm{m}$ and 2000 $\mu \mathrm{m})$ of BCP-R is shown in Figure $4 \mathrm{e}$. It could be seen that similar trends in the distribution of new bone exist among the different channels: no bone formation at the openings (i.e. at 0 and $7000 \mu \mathrm{m}$ on the $\mathrm{x}$-axis); bone formation always started at about $500 \mu \mathrm{m}$ far from both openings (i.e. at 500 and $6500 \mu \mathrm{m}$ on the $\mathrm{x}$-axis); high amount of bone formation was observed in the central part of the channels (i.e. between 750 and $6000 \mu \mathrm{m}$ on the x-axis). Furthermore, it appeared that higher amount of bone formed in the channels with $1 \mathrm{~mm}$ diameter rather than in the others, but no significant differences in the bone amount were seen since the standard deviation was high $(p>0.05)$. Table 2 shows the amount of bone formation in each channel of the BCP ceramics. In BCP-R ceramics: with the same diameter, the type of channel (i.e. crossing and non-crossing) does not affect the amount of bone formation ( $p>0.05)$; within the same type of channel, the diameter of the channel does not affect the amount of bone formation either $(p>0.05)$. However, the amount of bone formation was significantly affected by the surface roughness, where significantly higher amounts of bone formed in the channels of BCP-R $(p<0.01$ or $p<0.001)$. The histological overviews (Figures $4 \mathrm{a}$ and $4 \mathrm{~b}$ ) show that the channels of both BCP ceramics retained their shape after a 12-week implantation indicating no obvious resorption of both ceramics in vivo. The quantitative data confirmed this observation (Table 3 ) where no significant resorption was seen in both ceramics. In particular, the measurements showed no effect of the diameter or region (i.e. at the openings and in the central sector) of the channel on the ceramics resorption. 


\section{Chapter 6}

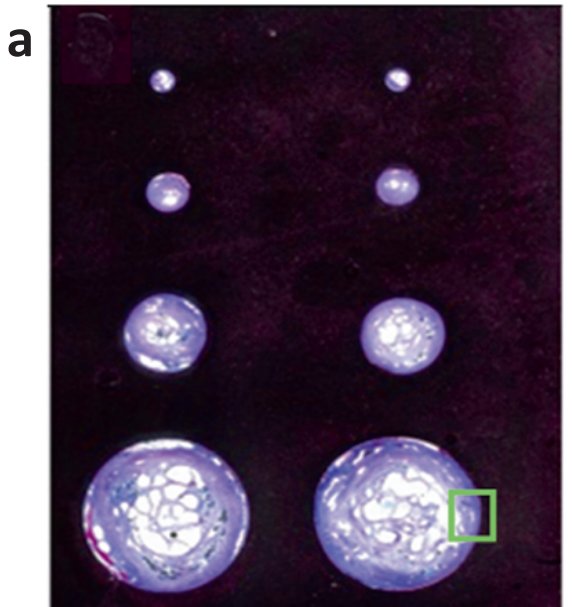

b

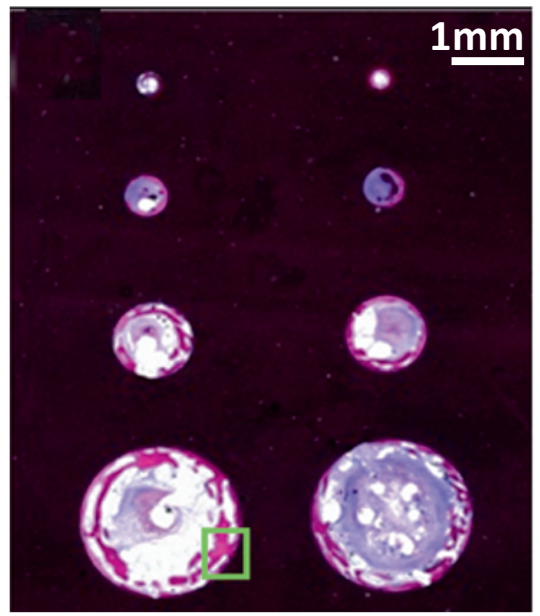

C
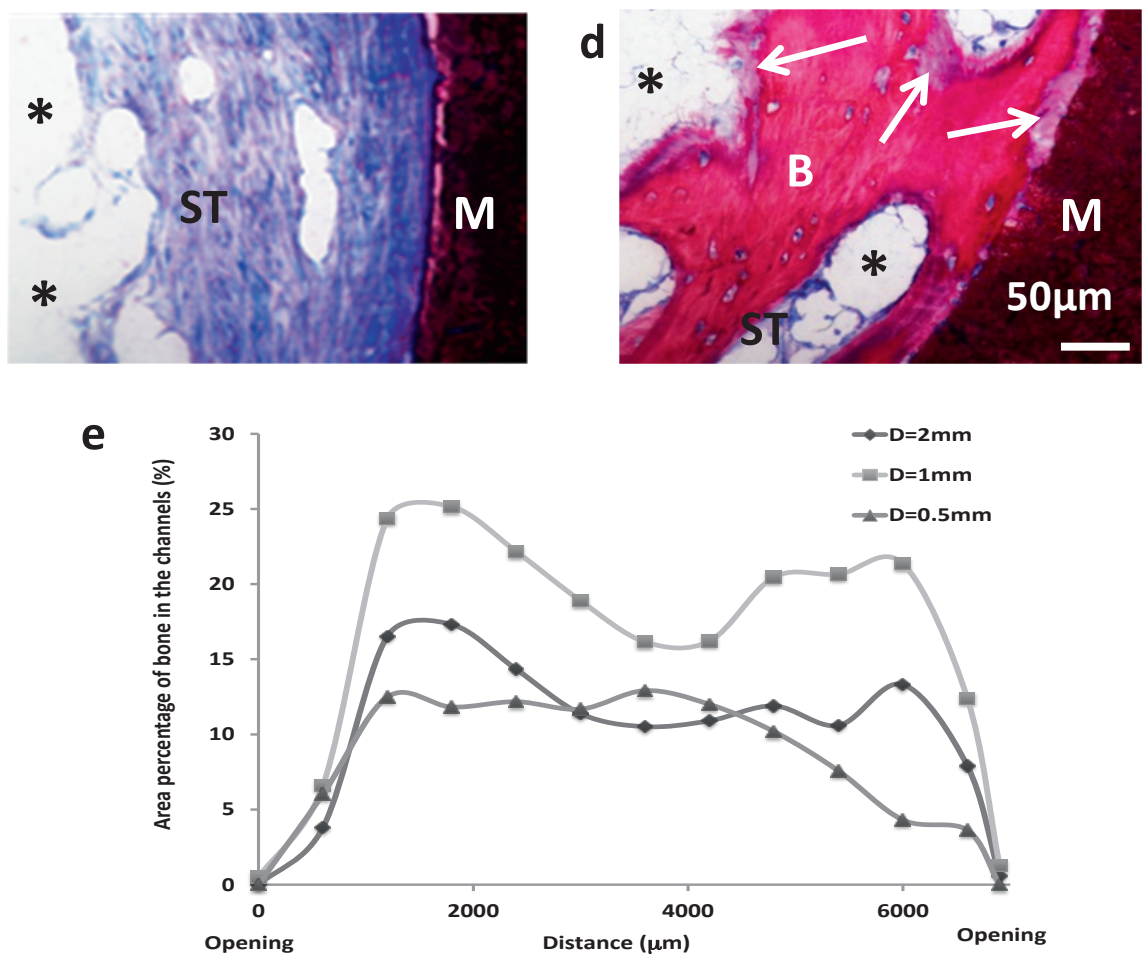

Figure 4. Histology of the BCP ceramics (a and c: BCP-S; $\boldsymbol{b}$ and $\boldsymbol{d}$ : $B C P-R$ ) after 12 weeks in muscle of dogs. The green rectangles in (a) and (b) highlight the areas observed with light microscope in (c) and (d). Figure (e) shows bone formation area along the crossing channels in BCP-R. Legend of symbols: ST stands for soft tissues, M for material, and B for bone. 
Table 2. A summary of the bone forming ability in vivo for the ceramics studied. It can be observed that the bone incidence as well as the newly formed bone volume were significantly higher in BCP-R than BCP-S when considering the case of closed channels (i.e. with only one opening). The presence of a second opening in the channels (i.e. two sides open case) did not significantly change the in vivo performance of BCP-R.

\begin{tabular}{ccccc}
\hline & Samples & & Bone incidence & Bone in pores \% vt. \\
\hline & & 2 & $100 \%$ & $11.0 \pm 3.8$ \\
& Non-crossing & 1 & $100 \%$ & $17.7 \pm 5.7$ \\
BCP-R & $(\varnothing: \mathrm{mm})$ & 0.5 & $100 \%$ & $7.9 \pm 9.4$ \\
& & 0.25 & $75 \%$ & $15.5 \pm 19.5$ \\
\cline { 2 - 5 } & & 2 & $100 \%$ & $14.3 \pm 5.8$ \\
& Crossing & 1 & $100 \%$ & $22.3 \pm 9.6$ \\
\hline \multirow{2}{*}{ BCP-S } & $(\varnothing: \mathrm{mm})$ & 0.5 & $87.5 \%$ & $6.8 \pm 9.4$ \\
\hline & & 2 & $50 \%$ & $0.6 \pm 1.1$ \\
& $(\varnothing: \mathrm{mm})$ & 0.5 & $62.5 \%$ & $0.9 \pm 2.4$ \\
& & 0.25 & $31.25 \%$ & $1.8 \pm 2.3$ \\
\hline
\end{tabular}

\subsection{Discussion}

In this study we used optimized BCP ceramics to investigate whether the surface architecture is playing a role in material-directed osteoinduction. We could minimize the number of possible influencing factors and make sure that the two ceramics differed only in their surface architecture. After a 12-week intramuscular implantation, BCP-R gave rise to abundant bone formation while BCP-S could induce the formation of a little amount of bone. The results presented herein thus indicate an important role of surface architecture in the osteogenesis process by CaP ceramics. 


\section{Chapter 6}

Chemistry $[21,22,42]$, macrostructure $[11,48]$ and microstructure $[16,49,50]$ have been suggested to play roles in material dependent osteoinduction. The possible influence of chemistry and macrostructure could be excluded in our study. The two BCP ceramics were made from the same CaP powder and, as shown in the XRD patterns, both BCP ceramics had the same composition with $80 \pm 3 \%$ wt. HA and $20 \pm 3 \%$ wt. $\beta$-TCP. This indicated that the different manufacture methods did not affect the final chemistry of the BCP ceramics. The macrostructure of the two BCP ceramic implants was kept equal: both BCP ceramics contained non-crossing channels with different diameters (i.e. $250 \mu \mathrm{m}, 500 \mu \mathrm{m}, 1000 \mu \mathrm{m}$ and $2000 \mu \mathrm{m}$ ), and the channels were created in the same positions in all the blocks.

Table 3. A summary of ceramic resorption in vivo (as measured for the non-crossing channels in both ceramics), which did not occur regardless of the ceramic type or channel diameter.

\begin{tabular}{cccccccccc}
\hline & & \multicolumn{2}{c}{ Peripheral region of the channels (Ф: mm) } & \multicolumn{4}{c}{ Central region of the channels (Ф: mm) } \\
& & $\mathbf{2}$ & $\mathbf{1}$ & $\mathbf{0 . 5}$ & $\mathbf{0 . 2 5}$ & $\mathbf{2}$ & $\mathbf{1}$ & $\mathbf{0 . 5}$ & $\mathbf{0 . 2 5}$ \\
\hline $\begin{array}{c}\text { Resortpion } \\
\text { rate } \\
\text { (\%) }\end{array}$ & BCP-R & $0.28 \pm 1.47$ & $-1.42 \pm 2.27$ & $-1.21 \pm 5.71$ & $-3.24 \pm 4.42$ & $0.23 \pm 2.27$ & $-0.26 \pm 3.34$ & $-1.99 \pm 5.10$ & $1.28 \pm 2.29$ \\
& BCP-S & $2.6 \pm 2.49$ & $3.49 \pm 3.5$ & $1.71 \pm 3.39$ & $-4.32 \pm 2.13$ & $0.09 \pm 1.16$ & $-0.11 \pm 2.75$ & $1.68 \pm 4.65$ & $1.34 \pm 1.49$ \\
\hline
\end{tabular}

The histological analysis showed that the newly formed bone was not on the surface of the implants, but it always formed inside the channels suggesting the important role of macrostructure in osteoinduction. This result is consistent with a previous study [19], where it was concluded that a concave structure is necessary for osteoinduction in CaP ceramics. To further investigate the effect of macrostructure dimension on bone formation, we fabricated both BCP ceramic implants with different channels' diameters. When analyzing the ceramics, no significant difference was observed in both the bone incidence and the bone amount among the channels having different diameter. It is worthy of notice that this fact was found for both BCP-R and BCP-S, and therefore the diameter of the channels did not seem to be essential for bone ingrowth in the tested range. However, the measured percentage of bone in the 12-week samples could not address the dynamic metabolism of the formed bone regarding its growth towards the center of the channels and its remodeling, therefore the possible influence of the channels' diameter on bone metabolism could not be 
excluded. It may well be that, at other time points such as later than 12 weeks, the smaller channels would have more bone fill than the larger ones as the latter will take longer to be fully colonized by bone tissue. Furthermore, we observed that there was no significant difference between crossing and non-crossing channels in BCP-R, which is in good agreement with a previous study [12] where it was claimed that interconnected macropores are not necessary for osteoinduction. However, the histological results showed that the incidence and amount of bone induced by BCP-S were lower than BCP-R regardless of the channel diameter (Table 2). Therefore, the surface microstructure may have played roles as main determinant of the osteoinductive potential of the BCP ceramics, which was already shown in several studies $[16,46,49]$. It is generally suggested that microporous $\mathrm{CaP}$ ceramics affects bone formation by virtue of its enlarged surface area since it would result in higher protein adsorption (e.g. BMP-2), higher $\mathrm{Ca}$ and $\mathrm{P}$ ions release and induced surface mineralization, or their combinations.

Both BCP-R and BCP-S used in this study had the same volume and equal surface area as shown in table 1. Therefore, the surface area was not the main factor influencing the bone forming potential. Meanwhile, the two BCP ceramics adsorbed similar amounts of proteins from BSA and FBS solutions, and they also could uptake rhBMP-2 from cell culture medium (Fig. 4). These results confirm the fact that surface area of $\mathrm{CaP}$ ceramics influences the protein adsorption. Having equal surface area, BCP-R and BCP-S adsorbed equivalent amounts of proteins regardless of the type of protein. The results also show that the protein adsorption could not be appointed as the main determinant for osteoinduction, since bone formation was different between BCP-R and BCP-S which adsorbed the same amount of proteins.

It has been reported that extracellular $\mathrm{Ca}$ and $\mathrm{P}$ significantly influenced the growth and osteogenic differentiation of MSCs [48], meanwhile the Ca and P supplementation promoted bone cell mineralization [16]. It has been also suggested that have been made the dissolution of $\mathrm{Ca}$ and $\mathrm{P}$ ions can be improved when biomaterials are pre-coated with $\mathrm{CaP}$ apatite layers. When titanium alloy was covered by an apatite layer, it could dissolve and release ions that can favor osteogenic differentiation of progenitor cells [51] indicating a key role in the CaP dissolution. It is therefore possible that the microporous CaP ceramics used in 


\section{Chapter 6}

our study initiated inductive bone formation via ion release during material resorption. However, no obvious resorption of both BCP ceramics was observed in this study. The reason could be that the TCP content in BCP ceramics was too low $(20 \%)$ or the implantation time was too short (12 week) to allow the resorption of the ceramics. Moreover, both BCP-R and BCP-S, instead of releasing, adsorbed similar amount of $\mathrm{Ca}$ and $\mathrm{P}$ ions from culture medium after 24 hours in vitro. Therefore, the degradation rate of the materials and the $\mathrm{Ca}$, $\mathrm{P}$ ions release was not the affecting factors in BCP-R ceramic-induced osteogenesis.

The ability to get surface mineralization has also been suggested being important in ectopic osteogenesis because ectopic bone formation was observed quite often in materials that can easily mineralize. Winter and Simpson reported that ectopic bone formation induced by a polymeric material occurred after surface mineralization of the implant [51]. Fujibayashi et al. studied the in vivo osteoinductivity of titanium implants, which could or not surface mineralize in vitro in SBF. The results demonstrated that those implants that induced an apatite layer formation in vitro had osteoinductive potential in vivo [35]. Similarly, Barbieri et al. reported the role of surface mineralization in vitro in material-directed bone induction. They prepared poly ( $D$, L-lactide) and nano-sized apatite composites with various apatite contents that were used to test in vivo osteoinduction. The results showed that only composites with at least $40 \%$ wt. apatite content could surface mineralize in vitro and induced bone formation in vivo [52]. Besides the spontaneous formation of mineralized layers by natural precipitation of ions from biological fluids, pre-coating biomaterials with CaP layers has shown to improve their biological activity. For example, polycaprolactone/TCP composites with hydroxyapatite-like coating allowed not only better adhesion and proliferation of porcine BMSCs as compared to non-coated counterparts [54] but also better osteogenic differentiation as the expression levels of various osteogenic indicators was improved. The authors suggested that such apatite-like coating layers would mimic the bone environment thus direct cells towards bone phenotypes [54]. When a polymer, i.e. polycaprolactone, was coated with a CaP layer and then cultured with osteoblasts [55], it could induce ectopic bone formation subcutaneously. The same study observed significant lower performances of the non-coated polymer indicating a crucial role of apatite-like layers in bone induction. However, our results showed that both BCP-R and BCP-S mineralized in 
vitro with similar trends, thus the possible influence of surface mineralization on their different osteoinductivity could be excluded.

Taken together, the role of surface architecture (as characterized by surface roughness) in heterotopic bone formation induced by CaP ceramics could be clearly seen in this study with the well-designed materials. In fact, previous studies already showed that osteoblast-like cells favor rougher surface to smooth surface in vitro [37-40]. It also has been demonstrated that microscale roughness with a high density of nanoscale features were superior to microscale roughness also in promoting osteogenic induction of the human osteosarcoma cell line MG63, determined by increased osteocalcin, osteoprotegerin and vascular endothelial growth factor. Moreover, Hu et al. examined the changes in hMSC proliferation and differentiation after growth on silk/tropoelastin materials with different content of human tropoelastin, together with different surface roughness and micro/nano-scale surface patterns. They found that higher surface roughness with micro/nano-scale surface patterns, and by increasing the content of human tropoelastin in the materials, enhanced the proliferation and osteogenic differentiation of hMSCs [36]. In our study, the different methods used to prepare the materials resulted in different micro-pore size (Figure 1b) and thus in different surface roughness (Figure 1e-h). The result herein showed a relationship between surface properties of materials and bone induction in vivo. Many biochemists and biologists induce osteogenic differentiation of cells by topography factors in vitro, but there is no well-understood way of controlling such factors in vivo. We demonstrated in this report a clear mechanism for osteoinduction based on the surface architecture providing an explanation for the control of bone induction by specific surface roughness. It is most probable that surface architecture could physically affect osteogenic differentiation of stem cells or undifferentiated inducible osteoprogenitor cells in ectopic sites and lead to the new heterotopic bone formation.

How the surface architecture of CaP ceramics drives osteogenesis in ectopic sites is not clear as yet. It should be mentioned that, upon injury due to the surgery, an inflammatory reaction is immediately triggered to limit the damage while blood vessels restrict to stop bleeding. During this stage, macrophage migration to the injured site and quick formation of fibrin-based platelet-rich clot may have happened [56, 57]. Macrophages and platelets, 


\section{Chapter 6}

together with endothelial cells in the damaged vessels, might have released various biomolecules (e.g. cytokines and several growth factors) prompting angiogenesis and migration of (stem) cells to the site $[56,57]$. In this study, the presence of the microstructured surface of BCP implants may have driven such (stem) cells towards osteogenic differentiation favoring the observed ectopic bone formation. To confirm this suggestive mechanism, a histological analysis of short-term implants, i.e. within few days from implantation, is recommended to analyze the blood clot formation and presence of inflammatory cells in future studies.

Recently it has been shown that scaffolds seeded with human mesenchymal progenitor cells and enriched with rhBMP-7 could ectopically form bone tissue that resembled the typical characteristics of a physiological bone, including a trabecular structure surrounded by a cortex-like layer [58]. We could not unequivocally identify the fat-like tissue present in the large channels of BCP-R (Figure $4 \mathrm{~d}$ ) as fat marrow and therefore it is not possible to claim that the observed bone can be considered as a complete organ bone in the current study. However, the use of histochemical stains is suggested in future studies to identify the possible presence of bone marrow and better understand if material-driven ectopic bone formation can lead to a complete functional bone tissue.

The optimized CaP ceramics allowed us to study whether the surface architecture of CaP ceramics could act as a cue for osteoinduction of CaP ceramics in vivo. Although we have not pinpointed a mechanism where surface architecture triggers ectopic bone formation of CaP ceramics, to the best of our knowledge it is the first work describing the significant role of a surface architecture in bone formation in vivo, instead of in vitro osteogenic differentiation of stem cells driven by surface characteristics as often documented in literature.

\subsection{Conclusions}

Given the same chemical composition, surface area per volume, proteins adsorption, ion release rate and surface mineralization ability, the surface architecture (i.e. surface roughness) affects bone forming ability of BCP ceramics, with a surface roughness of Ra $325.4 \pm 58.9 \mathrm{~nm}$ as a favorite scale for bone formation in this study. The results also showed that the percentage of bone volume in the channels was significantly affected by the surface 
architecture of the ceramics instead of the type (i.e. crossing vs. non-crossing) and size of the channels. Although the biological mechanism of ectopic osteogenesis of microporous CaP ceramics is far beyond understood, the current results demonstrate the surface roughness is a cue for material-induced bone formation. 


\section{Chapter 6}

\section{References}

[1] Kanzler B, Kuschert SJ, Liu YH, Mallo M. Hoxa-2 restricts the chondrogenic domain and inhibits bone formation during development of the branchial area. Development 1998;125:2587-2597.

[2] Gorski J. Distinctive distributions and properties of non-collagenous matrix proteins in lamellar vs. woven bone imply the existence of different underlying osteogenic mechanisms. Crit Rev Oral Biol Med 1998;9:201-223.

[3] Van der Stok J, van Lieshout EM, El-Massoudi Y, van Kralingen GH, Patka P. Bone substitutes in the Netherlands-A systematic literature review. Acta Biomater 2011;7:739-750.

[4] Ripamonti U. Osteoinduction in porous hydroxyapatite implanted in heterotopic sites of different animal models. Biomaterials 1998;17:31-35.

[5] Yang Z, Yuan H, Tong W, Zou P, Chen W, Zhang X. Osteogenesis in extraskeletally implanted porous calcium phosphate ceramics: variability among different kinds of animals. Biomaterials 1996;17:2131-2137.

[6] Yuan H, Kurashina K, de Bruijn JD, Li Y, de Groot K, Zhang X. A preliminary study on osteoinduction of two kinds of calcium phosphate ceramics. Biomaterials 1999;20:1799-1806.

[7] Siddappa R, Martens A, Doorn J, Leusink A, Olivo C, Licht R, van Rijn L, Gaspar C, Fodde R, Janssen F, van Blitterswijk CA, de Boer J. cAMP/PKA pathway activation in human mesenchymal stem cells in vitro results in robust bone formation in vivo. Proc Natl Acad Sci USA 2008;105:7281-7286

[8] Yamasaki H, Sakai H. Osteogenic response to porous hydroxyapatite ceramics under the skin of dogs. Biomaterials 1992;13:308-312.

[9] Klein C, de Groot K, Chen W, Li Y, Zhang X. Osseous substance formation induced in porous calcium phosphate ceramics in soft tissues. Biomaterials 1994;15:31-34.

[10] Ripamonti $U$. The morphogenesis of bone in replicas of porous hydroxyapatite obtained from conversion of calcium carbonate exoskeletons of coral. J Bone Joint Surg Am 1991;73:692-703.

[11] Ripamonti U, Richter PW, Thomas ME. Self-inducing shape memory geometric cues embedded within smart hydroxyapatite-based biomimetic matrices. Plast Reconstr Surg 2007;120:1796-1807.

[12] Ripamonti U, Crooks J, Khoali L, Roden L. The induction of bone formation by coral-derived calcium carbonate/hydroxyapatite constructs. Biomaterials 2009;30:1428-1439.

[13] Yuan H, van Blitterswijk CA, de Groot K, de Bruijn JD. Cross-species comparison of ectopic bone formation in biphasic calcium phosphate (BCP) and hydroxyapatite (HA) scaffolds. Tissue Eng 2006;12:1607-1615.

[14] Gosain AK, Song L, Riordan P, Amarante MT, Nagy PG, Wilson CR, et al. A 1-year study of osteoinduction in hydroxyapatite-derived biomaterials in an adult sheep model: Part I. Plast Reconstr Surg 2002;109:619-630.

[15] Habibovic P, Yuan H, van der Valk CM, Meijer G, van Blitterswijk CA, de Groot K. 3D microenvironment as essential element for osteoinduction by biomaterials. Biomaterials 2005;26:3565-3575.

[16] Habibovic P, Sees TM, van den Doel MA, van Blitterswijk CA, de Groot K. Osteoinduction by biomaterialsphysicochemical and structural influences. J Biomed Mater Res A 2006;77:747-762.

[17] Yuan H, Yang Z, Li Y, Zhang X, de Bruijn JD, de Groot K. Osteoinduction by calcium phosphate biomaterials. J Mater Sci Mater Med 1998;9:723-726.

[18] Yuan H, Yang Z, de Bruijn JD, de Groot K, Zhang X. Material-dependent bone induction by calcium phosphate ceramics: a 2.5-year study in dog. Biomaterials 2001;22:2617-2623.

[19] Yuan H, de Bruijn JD, Li Y, Feng J, Yang Z, de Groot K, et al. Bone formation induced by calcium phosphate ceramics in soft tissue of dogs: a comparative study between porous $\alpha$-TCP and $\beta$-TCP. J Mater Sci Mater Med $2001 ; 12: 7-13$

[20] Kondo N, Ogose A, Tokunaga K, Umezu H, Arai K, Kudo N, et al. Osteoinduction with highly purified $\beta$ tricalcium phosphate in dog dorsal muscles and the proliferation of osteoclasts before heterotopic bone formation. Biomaterials 2006;27:4419-4427.

[21] Yuan H, Fernandes $H$, Habibovic $P$, de Boer J, Barradas A, de Ruiter A, et al. Osteoinductive ceramics as a synthetic alternative to autologous bone grafting. Proc Natl Acad Sci USA 2010;107:13614-13619.

[22] Yuan H, van Blitterswijk CA, de Groot K, de Bruijn JD. A comparison of bone formation in biphasic calcium phosphate $(\mathrm{BCP})$ and hydroxyapatite $(\mathrm{HA})$ implanted in muscle and bone of dogs at different time periods. J Biomed Mater Res A 2006;78:139-147. 
[23] le Nihouannen D, Saffarzadeh A, Gauthier O, Moreau F, Pilet P, Spaethe R, et al. Bone tissue formation in sheep muscles induced by a biphasic calcium phosphate ceramic and fibrin glue composite. J Mater Sci Mater Med 2008;19:667-675.

[24] Yuan H, van den Doel M, Li S, van Blitterswijk CA, de Groot K, de Bruijn JD. A comparison of the osteoinductive potential of two calcium phosphate ceramics implanted intramuscularly in goats. J Mater Sci Mater Med 2002;13:1271-1275

[25] Habibovic P, van der Valk C, van Blitterswijk CA, de Groot K, Meijer G. Influence of octacalcium phosphate coating on osteoinductive properties of biomaterials. J Mater Sci Mater Med 2004;15:373-380.

[26] Li JP, Habibovic P, Yuan H, van den Doel M, Wilson CE, de Wijn JR, et al. Biological performance in goats of a porous titanium alloy-biphasic calcium phosphate composite. Biomaterials 2007;28:4209-4218.

[27] Habibovic P, Kruyt MC, Juhl MV, Clyens S, Martinetti R, Dolcini L, et al. Comparative in vivo study of six hydroxyapatite-based bone graft substitutes. J Orth Res 2008;26:1363-1370.

[28] Kurashina K, Kurita H, Wu Q, Ohtsuka A, Kobayashi H. Ectopic osteogenesis with biphasic ceramics of hydroxyapatite and tricalcium phosphate in rabbits. Biomaterials 2002;23:407-412.

[29] Habibovic P, Yuan H, van den Doel M, Sees TM, van Blitterswijk CA, de Groot K. Relevance of osteoinductive biomaterials in critical-sized orthotopic defect. J Orth Res 2006;24:867-876.

[30] Ripamonti U, Crooks J, Tucker M, Sampath T, Rueger D, Reddi A. Long-term evaluation of bone formation by osteogenic protein 1 in the baboon and relative efficacy of bone-derived bone morphogenetic proteins delivered by irradiated xenogeneic collagenous matrices. J Bone Min Res 2000;15:1798-1809.

[31] Duneas N, Crooks J, Ripamonti U. Transforming growth factor- $\beta$ 1: induction of bone morphogenetic protein genes expression during endochondral bone formation in the baboon, and synergistic interaction with osteogenic protein-1 (BMP-7). Growth Factors 1998;15:259-277.

[32] Ripamonti U, Heliotis M, Rueger D, Sampath T. Induction of cementogenesis by recombinant human osteogenic protein-1 (hOP-1/BMP-7) in the baboon (Papio ursinus). Arch Oral Biol 1996;41:121-126.

[33] Barradas A, Fernandes HA, Groen N, Chai YC, Schrooten J, van de Peppel J, et al. A calcium-induced signaling cascade leading to osteogenic differentiation of human bone marrow-derived mesenchymal stromal cells. Biomaterials 2012;33:3205-3215.

[34] Geesink R, de Groot K, Klein C. Bonding of bone to apatite-coated implants. J Bone Joint Surg $\mathrm{Br}$ 1988;70:17-22.

[35] Fujibayashi S, Neo M, Kim HM, Kokubo T, Nakamura T. Osteoinduction of porous bioactive titanium metal. Biomaterials 2004;25:443-450.

[36] Hu X, Park SH, Gil ES, Xia XX, Weiss AS, Kaplan DL. The influence of elasticity and surface roughness on myogenic and osteogenic-differentiation of cells on silk-elastin biomaterials. Biomaterials 2011;32:8979-8989

[37] Anselme K, Bigerelle M. Topography effects of pure titanium substrates on human osteoblast long-term adhesion. Acta Biomater 2005;1:211-22.

[38] Bowers KT, Keller JC, Randolph BA, Wick DG, Michaels CM. Optimization of surface micromorphology for enhanced osteoblast responses in vitro. Int J Oral Maxillofac Implants 1992;7:302-310.

[39] Kommireddy DS, Sriram SM, Lvov YM, Mills DK. Stem cell attachment to layer-by-layer assembled $\mathrm{TiO}_{2}$ nanoparticle thin films. Biomaterials 2006;27:4296-4303.

[40] Zhao G, Raines A, Wieland M, Schwartz Z, Boyan B. Requirement for both micron-and submicron scale structure for synergistic responses of osteoblasts to substrate surface energy and topography. Biomaterials 2007;28:2821-2829.

[41] Mendonça G, Mendonça D, Simões LGP, Araújo AL, Leite ER, Duarte WR, et al. The effects of implant surface nanoscale features on osteoblast-specific gene expression. Biomaterials 2009;30:4053-4062.

[42] Oh S, Brammer KS, Li Y, Teng D, Engler AJ, Chien S, et al. Stem cell fate dictated solely by altered nanotube dimension. Proc Natl Acad Sci USA 2009;106:2130-2135.

[43] Dalby MJ, McCloy D, Robertson M, Agheli H, Sutherland D, Affrossman S, et al. Osteoprogenitor response to semi-ordered and random nanotopographies. Biomaterials 2006;27:2980-2987.

[44] Kilian KA, Bugarija B, Lahn BT, Mrksich M. Geometric cues for directing the differentiation of mesenchymal stem cells. Proc Natl Acad Sci USA 2010;107:4872-4877.

[45] Dalby MJ, Gadegaard N, Tare R, Andar A, Riehle MO, Herzyk P, et al. The control of human mesenchymal cell differentiation using nanoscale symmetry and disorder. Nature Mater 2007;6:997-1003. 


\section{Chapter 6}

[46] Fu J, Wang YK, Yang MT, Desai RA, Yu X, Liu Z, et al. Mechanical regulation of cell function with geometrically modulated elastomeric substrates. Nature Methods 2010;7:733-736.

[47] Kokubo T, Takadama H. How useful is SBF in predicting in vivo bone bioactivity? Biomaterials 2006;27:29072915.

[48] Barradas AMC, Yuan H, van Blitterswijk CA, Habibovic P. Osteoinductive biomaterials: current knowledge of properties, experimental models and biological mechanisms. Eur Cell Mater 2011;21:407-429.

[49] Curtis A, Wilkinson C. Nantotechniques and approaches in biotechnology. Trends in Biotechn 2001;19:97101

[50] Galli C, Piemontese M, Lumetti S, Manfredi E, Macaluso G, Passeri G. The importance of WNT pathways for bone metabolism and their regulation by implant topography. Eur Cells Mater 2012;24:46-59.

[51] Chai YC, Truscello S, van Bael S, Luyten FP, Vleugels J, Schrooten J. Perfusion electrodeposition of calcium phosphate on additive manufactured titanium scaffolds for bone engineering. Acta Biomater 2011;7: 2310-2319.

[52] Winter GD, Simpson BJ. Heterotopic bone formation in a synthetic sponge in the skin of young pigs. Nature 1969;223:88-90.

[53] Barbieri D, Renard AJS, de Bruijn JD, Yuan H. Heterotopic bone formation by nano-apatite containing poly(D,L-lactide) composites. Eur Cells Mater 2010;19:252-261.

[54] Arafat MT, Lam CXF, Ekaputra AK, Wong SY, Li X, Gibson I. Biomimetic composite coating on rapid prototyped scaffolds for bone tissue engineering. Acta Biomater 2011;7:809-820.

[55] Vaquette C, Ivanovski S, Hamlet SM, Hutmacher DW. Effect of culture conditions and calcium phosphate coating on ectopic bone formation. Biomaterials 2013;34:5538-5551.

[56] Anderson JM, Rodriguez A, Chang DT. Foreign body reaction to biomaterials. Semin Immunol 2008;20:86100.

[57] Davies JE. Understanding peri-implant endosseous healing. J Dent Ed 2003;67:932-949.

[58] Holzapfel BM, Wagner F, Loessner D, Holzapfel NP, Thibaudeau L, Crawford R, Ling MT, Clements JA, Russell PJ, Hutmacher DW. Species-specific homing mechanisms of human prostate cancer metastasis in tissue engineered bone. Biomaterials 2014;35:4108-4115. 


\section{Chapter 7}

General discussion and main conclusions 

Autologous bone graft is the gold standard treatment for large bone defects, but the drawbacks of additional surgical time and limited availability rationalized the development of synthetic bone graft substitutes [1]. In this sense, calcium phosphate (CaP) ceramics are frequently used because their chemical composition is similar to the mineral component of bone [1-3]. In general, CaP ceramics are biocompatible and osteoconductive, which can provide excellent osteointegration of the implants with the host bone. Moreover, during the last decades a specific group of CaP ceramics has shown to have also the potential to trigger bone formation when implanted in non-bony sites, a phenomenon defined as osteoinduction [4-11]. In particular, it has been shown that the osteoinductive potential varies with the physicochemical properties of CaP ceramics [12]. More specifically, chemistry, macrostructure and surface microstructure have been suggested the essential factors determining the osteoinduction potential in CaP ceramics [13-18]. By keeping the same chemistry in individual studies, the possible role of several physical properties (e.g. macrostructure and surface microstructure) in CaP ceramic-driven osteoinduction process was addressed in the current thesis.

\subsection{Macrostructure in osteoinduction}

It is widely suggested that the macrostructure of CaP ceramics allows blood vessel ingrowth and creates a protected environment to form new bone. Osteoinduction was often observed in CaP ceramics having either interconnected macropores or well-defined macro-concavities [18]. In this thesis, the role of macrostructure of CaP ceramics in osteoinduction was investigated in Chapter 6. BCP plates with macro-scaled channels having various diameters $(0.25,0.5,1.0$ and $2.0 \mathrm{~mm})$ were implanted in the muscle of dogs. No significant differences in bone formation were observed among the channels, showing that macrostructures at the range of 250 to $2000 \mu \mathrm{m}$ do not affect osteoinductive potentials of CaP ceramics. Surprisingly, a clear difference in bone formation was noticed between BCP ceramics having different surface microstructures: the rougher the surface structure, the higher osteoinductive potential. 


\section{Chapter 7}

\subsection{The scale of surface microstructure in osteoinduction}

Surface microstructure is a property of CaP ceramics determined by the presence of micropores and micrograins and their dimension. The importance of surface microstructure in osteoinduction has been highlighted by several research groups, as indicated by the influence of micropores of CaP ceramics on osteoinduction [13]. For instance, Yamasaki and Sakai demonstrated that the presence of micropores is a key material parameter triggering osteoinduction $[4,6,13,14]$. They have shown that, once implanted in the dorsal muscle of canines, microporous HA was osteoinductive but the dense one was not. This finding was further confirmed by Yuan and Habibovic, who reported that CaP ceramics with surface microstructure were osteoinductive while those without were not $[4,6,14]$.

In addition to the presence of surface microstructure, the current thesis has shown the importance of the surface microstructure dimension of CaP ceramics in osteoinduction. In Chapters 3, and 6, CaP ceramics having various sizes of the surface grains and micropores, and different levels of surface roughness, were prepared and ectopically evaluated in vivo (Table 1).

Table 1. The surface microstructure of osteoinductive and non-osteoinductive CaP ceramics, osteoinductive $(\mathrm{Y})$ and non-osteoinductive/little osteoinductive (N).

\begin{tabular}{|c|c|c|c|c|c|c|c|}
\hline \multirow{2}{*}{\multicolumn{2}{|c|}{ CaP Ceramics }} & \multirow{2}{*}{ Chapter } & \multirow{2}{*}{$\begin{array}{l}\text { Osteoinductive } \\
\text { capacity }\end{array}$} & \multirow{2}{*}{$\begin{array}{l}\text { Grain size } \\
(\mu \mathrm{m})\end{array}$} & \multirow{2}{*}{$\begin{array}{l}\text { Micropore } \\
\text { size }(\mu \mathrm{m})\end{array}$} & \multicolumn{2}{|c|}{ Surface roughness $(\mu \mathrm{m})$} \\
\hline & & & & & & $\mathbf{R a}$ & $\mathbf{R q}$ \\
\hline \multirow{3}{*}{ TCP } & TCP-S & & $\mathbf{Y}$ & $0.90 \pm 0.25$ & $0.67 \pm 0.30$ & $482.8 \pm 73$ & $590 \pm 90.1$ \\
\hline & & Chapter 3 & & & & & \\
\hline & TCP-B & & N & $3.40 \pm 0.82$ & $1.70 \pm 0.63$ & $744.3 \pm 395$ & $630 \pm 19.5$ \\
\hline \multirow{3}{*}{ BCP } & BCP-R & & $\mathbf{Y}$ & $0.91 \pm 0.27$ & $0.83 \pm 0.67$ & $325.4 \pm 58.9$ & $397.4 \pm 65.6$ \\
\hline & & Chapter 6 & & & & & \\
\hline & BCP-S & & N & $0.93 \pm 0.26$ & $0.58 \pm 0.27$ & $231.6 \pm 35.7$ & $294.7 \pm 46.1$ \\
\hline
\end{tabular}

Chapters 2 and 3 show that, in the muscle of the canines, TCP-S with submicron surface structure (i.e. TCP-S granules: grain size of $0.99 \pm 0.20 \mu \mathrm{m}$ and micropore size of $0.65 \pm 0.25 \mu \mathrm{m}$; TCP-S discs: grain size of $0.90 \pm 0.25 \mu \mathrm{m}$ and micropore size of $0.67 \pm 0.30 \mu \mathrm{m}$ ) could induce de novo bone formation, but TCP-B having lager surface structure (i.e. TCP-B granules: grain size 
of $3.08 \pm 0.52 \mu \mathrm{m}$ and micropore size of $1.58 \pm 0.65 \mu \mathrm{m}$; TCP-B discs: grain size of $3.40 \pm 0.82$ $\mu \mathrm{m}$ and micropore size of $1.70 \pm 0.63 \mu \mathrm{m})$ could not. It is interesting that this result was reproducible regardless of the implants form of porous granules (Chapter 2), dense discs (Chapter 3) or porous cylinders [16]. Therefore, it may be concluded that, to get osteoinductive $\mathrm{CaP}$ ceramics, the size of surface grains and micropores should be kept at the submicron scale. CaP ceramics having too large surface structures (e.g. micron scaled grains and micropores) resulted non- osteoinductive.

Even though the grains and micropores were controlled at the submicron scale, the $\mathrm{CaP}$ ceramics may still not be osteoinductive. As shown in Chapter 6, both BCP-R and BCP-S had submicron scaled grains and pores, but only BCP-R having a rougher surface gave rise to inductive bone formation, indicating the necessity of a sufficiently rough surface. While a comparison between the data in Chapter 3 and 6 did not allow us making a direct correlation between surface roughness and inductive bone formation capacity because TCP-B, even though it had a rougher surface than TCP-S, did not induce bone formation while TCP-S did.

The results summarized in Chapter 3 and 6 have shown the important role of surface structure scale in the phenomenon of $\mathrm{CaP}$ ceramics-driven osteoinduction. In particular, they indicate that osteoinductive CaP ceramics must have a submicron scaled surface structure (i.e. grains and micropores) and a narrow surface roughness range (e.g. 325.4 \pm 58.9 $\mu \mathrm{m}<\mathrm{Ra}<482.8 \pm 73.0 \mu \mathrm{m}, 397.4 \pm 65.6 \mu \mathrm{m}<\mathrm{Rq}<590 \pm 90.1 \mu \mathrm{m}$ ) (Table 1).

\subsection{The physical role of surface microstructure in osteoinduction}

Although the importance of surface microstructure in inductive bone formation was often identified, how surface structure plays its role in inductive bone formation in vivo remains unclear. Based on the biological functions of chemical cues, three theories have been previously proposed to explain how the physicochemical properties of CaP to induce de novo bone formation in vivo. 1) The $\mathrm{Ca}$ and $\mathrm{P}$ ion release from the CaP ceramics (soluble factors) $[19,20] ; 2)$ the precipitation of a carbonated apatite layer onto the surface of CaP ceramics [4, 21-23]; 3) local concentration of biochemical factors (e.g. bone morphogenetic proteins (BMPs)) $[7,14,20,24]$. However, another suggested physicochemical theory cannot be ignored: the surface topography of biomaterials may direct stem cell differentiation 


\section{Chapter 7}

leading to the cellular synthesis of new bone [25]. Using both an in vitro model of human bone marrow mesenchymal stromal cells (hBMSCs) and an in vivo osteoinduction study model, the work of this thesis took the challenge to separate the role of physical cues from that of chemical cues in inductive bone formation.

BMSCs have been extensively investigated during the last ten years, because they are able to differentiate into various cell phenotypes including chondrocytes, myoblasts, adipocites and osteoblasts [20, 26-30]. A crucial role of osteogenic differentiation of BMSCs has been proposed for the osteoinduction process by CaP ceramics [31]. It was also reported that BMSCs can migrate from femoral bone towards osteoinductive CaP ceramic implants via the blood circulation in a canine model [31]. Using hBMSCs as an in vitro model, the cellular response to the scale of surface structure of CaP ceramics was investigated.

In Chapter 2 hBMSCs were cultured, in the absence of osteogenic growth factors, on two types of 3D TCP granules, which had equivalent chemistry and similar microporosity but different dimension of their surface structures. The results showed enhanced cell proliferation and osteogenic differentiation of hBMSCs seeded on submicron structured TCP$\mathrm{S}$ as compared to those on micron structured TCP-B. These findings suggest that the submicron size of surface structure of TCP-S provided a more suitable environment for the osteogenic differentiation of hBMSCs in vitro, which clearly indicate the crucial importance of the scale of surface structure. These observations were further confirmed by the studies described in Chapter 3, where 2D TCP-S and TCP-B discs were used to detect the cellular behavior of hBMSCs.

However, the findings in Chapter 2 still do not completely prove the physical role of surface structure in osteoinduction, because the different size of surface structure of TCP particles affected on the chemical cues (i.e. Ca ion in the medium, protein adsorption and precipitation of carbonate apatite layer) as well. It is not easy to change surface structure without generating differences in chemical cues. Therefore, to demonstrate that the surface structure could play a direct role in osteoinduction, the role of the chemical cues associated to changes of the surface structure must be excluded. 
To isolate the effect of soluble factors in osteogenic differentiation of hBMSCs, trans-well plate culture system was further applied in Chapter 2. In this in vitro system, cells were cultured on the bottom of the plate while TCP-S and TCP-B granules were loaded onto the membrane. A higher ALP activity of hBMSCs was observed in the medium containing TCP-B, which also showed a higher concentration of calcium ions as compared to TCP-S. These results are in line with a previous study where enhanced osteogenic differentiation of hBMSCs was due to a high extracellular calcium concentration [19]. However, when cells were cultured directly on the surface of the granules, TCP-S enhanced osteogenic differentiation instead of TCP-B where higher concentration of $\mathrm{Ca}$ ions in the medium was expected. This indicates that, in the 3D cell culture system, the soluble calcium ion is not the key factor to trigger osteogenic differentiation of hBMSCs in vitro.

Due to the large surface area, which results in more precipitation of calcium and phosphate ions, the concentration of calcium ions in the medium was lower in the case of TCP-S than TCP-B in Chapter 2. Again, in Chapter 4, because of the large surface of TCP-based materials than glass-based materials, the concentration of both $\mathrm{Ca}$ and $\mathrm{P}$ ions were lower in the medium containing TCP-based materials than that containing glass-based materials. However, ALP activity of hBMSCs was much higher on TCP-based materials than glass-based materials. These results further confirm that the soluble ions may not be appointed as the main determinant for osteoinduction of CaP ceramics.

However, excluding the possible role of soluble ions in osteoinduction is still not enough to fully prove the physical role of the surface structure in osteoinduction. Other material properties may also determine the bone induction, for example the protein adsorption and the precipitation of $\mathrm{Ca}$ and $\mathrm{P}$ ions. It is well known that changes in the surface chemistry in turn affect protein adsorption and ion precipitation [32]. Therefore, Chapter 4 was designed to exclude the possible influence of protein adsorption and precipitation of $\mathrm{Ca}$ and $\mathrm{P}$ ions on osteoinduction. To do so, either carbon or gold coating was applied onto TCP-based and glass-based materials. The results showed no significant differences in the morphology, proliferation and osteogenic differentiation of hBMSCs on TCP-based materials irrespective of coatings. However, when comparing the TCP-based material with the glass-based materialds, a significantly higher osteogenic differentiation of hBMSCs could be observed on 


\section{Chapter 7}

TCP-based materials than on glass-based materials regardless of the surface chemistry changes induced by the coatings. These results thereby suggest that protein adsorption and ion precipitation may not be crucial determinants of the osteoinductive potential and therefore the surface chemistry may be a flexible parameter when the surface structure is maintained. Taken together, these results point out that the surface structure could directly initiate osteogenic differentiation of hBMSCs via surface topography and indicate the physical role of surface structure in osteoinduction.

Chapter 6 shows more clearly the primary necessity of a specific surface structure in osteoinduction as compared to protein adsorption, ion exchange and surface reprecipitation. BCP-R and BCP-S having equal surface area per volume were precisely designed and produced, therefore the constancy of protein adsorption, release and precipitation of calcium and phosphate ions was expected and confirmed from the experimental tests. Histological analysis showed that abundant de novo bone was formed in BCP-R implants with a rougher surface structure, but only little bone was formed in BCP-S implants with a smoother surface structure. So, the observed difference in bone forming potential of the two studied BCP ceramics was attributed to the surface structure of BCP ceramic itself and not to the chemical cues.

\subsection{How surface structure initiates osteogenic differentiation of hBMSCs}

It has long been established that cells undergo a wide variety of morphological changes during the process of lineage commitment $[33,34]$. Differentiation was often reported to be associated with changes in cell morphology, and, in turn, cell morphology regulates the cellular differentiation as well $[35,36]$. Primary cilia have been reported to have the capacity to transduce both mechanical and biochemical signals and convert them into intracellular signals controlling a wide variety of processes during the development and tissue homeostasis $[37,38]$. It is possible that the primary cilium is also involved in the osteogenic differentiation of hBMSCs when responding to surface structure.

To address how the surface structure initiates osteogenic differentiation of MSCs, the response of hBMSCs to TCP ceramics was evaluated with respect to cell morphological changes and the development of primary cilia (Chapter 3). The development of primary cilia 
in hBMSCs can be affected by several experimental settings. For example, dexamethasone did not affect much the incidence of primary cilia, but it greatly influenced their length. We have observed (Chapter 3 ) that the surface structure significantly regulated both the incidence and length of primary cilia. When taking the primary cilia length as the osteogenic marker of hBMSCs, the influence of the experimental settings was seen. First, the surface structure at the submicron-scale could have the same osteogenic capacity of dexamethasone (TCP-S BM vs Glass OM). Secondly, when cells were cultured in BM, the role of surface structure dimension on osteogenic differentiation of hBMSCs could be seen, where the submicron scaled surface resulted superior to that at the micron scale.

The data obtained in Chapter 3 clearly suggest that the enhanced osteogenic differentiation of hBMSCs could be due to the cell morphology change and the development of primary cilia stimulated by the submicron surface structured CaP ceramics. The elongation of primary cilia may mediate osteogenic differentiation via the associated proteins of polycystin-1 (PC1), polycystin-2 (PC2) and intraflagellar transport protein-88 (IFT88) [39]. Furthermore, primary cilia could also affect a number of osteogenic signaling factors, such as Wnt [40], runx2 [39], TGF- $\beta[38,39]$ and hedgehog pathway [41].

\subsection{How surface structure plays roles in bone regeneration}

Osteoinductive CaP ceramics not only give rise to inductive bone formation in ectopic sites, but also enhance bone regeneration in orthotopic sites [4, 20, 42]. Bone regeneration normally initiates with inflammation and continues with angiogenesis, bone morphogenesis and bone remodeling [43]. After implantation of an osteoinductive CaP ceramic, wound healing is at its acute inflammatory stage before bone formation starts. It has been shown that anti-inflammatory chemicals inhibit inductive bone formation of osteoinductive CaP ceramics, indicating that inflammatory cells (e.g. macrophages) respond to the surface structure and produce osteogenic cytokines that may trigger osteoinduction in submicronstructured CaP ceramics [16]. In addition, it has also been reported that, during bone remodeling, cells responsible for bone resorption respond to the surface structure differently [44]. Human CD14+ monocytes could be differentiated into functional osteoclasts 


\section{Chapter 7}

on submicron-structured CaP ceramic (TCP-S), but not on micro-structured CaP ceramic (TCP-B) [45].

In this thesis, the response of various cells related to the osteogenesis in CaP ceramics was investigated. MC3T3-E1 (osteoprogenitors), SV-HFO (pre-osteoblasts), MG63 (osteoblasts) and SAOS-2 (mature osteoblasts) were systematically selected and tested according to their maturation stage (Chapter 5). Importantly, bone regenration requires not only the osteogenic of osteoblasts but also the recruitment and/or migration of a potentially osteogenic cell population and the differentiation of this population into osteogenic cells. Proposed inducible cells [31, 46-48] of hBMSCs (Chapter 2), HBVP (pericytes, Chapter 5) and C2C12 (myoblasts, Chapter 5) were also evaluated in this thesis. The results showed that surface structure of CaP ceramics influenced cellular behaviors at different mature stages in different manners - proliferation, osteogenic differentiation or both of them. As compared to the micron scale surface structure, the surface structure at submicron scale (TCP-S) promotes osteogenic differentiation of inducible cells (i.e. hBMSCs, C2C12), osteoprogenitors, pre-osteoblasts but not osteoblasts. However the proliferation of osteoblasts was promoted by the submicron scaled surface structure, although the osteogenic potential of osteoblasts was not enhanced.

\subsection{Future perspectives}

In addition to the proposed osteoinduction determinants of soluble factors, apatite formation and protein adsorption, this thesis provided the evidence of the osteogenic function of surface topographical cues.

By carefully designing the materials, we demonstrated that the surface structure dimension is crucial for osteoinduction, could physically trigger osteogenic differentiation of stem cells and give inductive bone formation.

However, our studies were conducted mainly with CaP ceramic materials, and therefore it is impossible to completely exclude the role of ion release, apatite formation and protein adsorption in the process of ceramic-driven osteoinduction. The surface structure design on materials with chemistry (e.g. non-CaP materials) would be a strategy for the future to 
evaluate if the surface topography is the predominant factor in osteoinduction of synthetic materials.

The molecular mechanism of stem cell responding to the surface structure to undergo osteogenic differentiation would be another interesting future direction to discover the biological mechanism of osteoinductive materials. Admittedly, the question about which cells in ectopic sites are responsible for material-related osteoinduction is not answered as yet. Bone morphogenesis in implants following an ectopic implantation may not be as simple as a solely osteogenic differentiation of stem cell. Inflammatory and osteoclastogenesis could be affected by osteoinductive materials as well $[44,45]$. A careful study design to explore the role of inflammation and osteoclastogenesis during the osteoinduction process would give us more insights on the biological mechanism of material-induced bone formation. 


\section{References}

[1] Bohner M, Galea L, Doebelin N. Calcium phosphate bone graft substitutes: Failures and hopes. Journal of the European Ceramic Society. 2012;32:2663-71.

[2] Jarcho M. Calcium phosphate ceramics as hard tissue prosthetics. Clinical orthopaedics and related research. 1981;157:259-78.

[3] Samavedi S, Whittington AR, Goldstein AS. Calcium phosphate ceramics in bone tissue engineering: a review of properties and their influence on cell behavior. Acta Biomaterialia. 2013;9:8037-45.

[4] Habibovic P, Sees TM, van den Doel MA, van Blitterswijk CA, de Groot K. Osteoinduction by biomaterialsphysicochemical and structural influences. Journal of Biomedical Materials Research Part A. 2006;77:747-62.

[5] Habibovic P, Van der Valk C, Van Blitterswijk C, De Groot K, Meijer G. Influence of octacalcium phosphate coating on osteoinductive properties of biomaterials. Journal of materials science Materials in medicine. 2004;15:373-80.

[6] Habibovic P, Yuan H, van den Doel M, Sees TM, van Blitterswijk CA, de Groot K. Relevance of Osteoinductive Biomaterials in Critical-Sized Orthotopic Defect. Journal of Orthopaedic Research. 2006;24:867-76.

[7] Habibovic P, Yuan H, Van Der Valk CM, Meijer G, Van Blitterswijk CA, De Groot K. 3D microenvironment as essential element for osteoinduction by biomaterials. Biomaterials. 2005;26:3565-75.

[8] Ripamonti U. Osteoinduction in porous hydroxyapatite implanted in heterotopic sites of different animal models. Biomaterials. 1996;17:31-5.

[9] Ripamonti U, Crooks J, Khoali L, Roden L. The induction of bone formation by coral-derived calcium carbonate/hydroxyapatite constructs. Biomaterials. 2009;30:1428-39.

[10] Yuan H, De Bruijn J, Li Y, Feng J, Yang Z, De Groot K, et al. Bone formation induced by calcium phosphate ceramics in soft tissue of dogs: a comparative study between porous $\alpha-T C P$ and $\beta-T C P$. Journal of materials science Materials in medicine. 2001;12:7-13.

[11] Yuan H, Van Den Doel M, Li S, Van Blitterswijk C, De Groot K, De Bruijn J. A comparison of the osteoinductive potential of two calcium phosphate ceramics implanted intramuscularly in goats. Journal of materials science Materials in medicine. 2002;13:1271-5.

[12] Barradas AM, Yuan H, van Blitterswijk CA, Habibovic P. Osteoinductive biomaterials: current knowledge of properties, experimental models and biological mechanisms. European cells \& materials. 2011;21:407-29; discussion 29.

[13] Yamasaki H, Sakai H. Osteogenic response to porous hydroxyapatite ceramics under the skin of dogs. Biomaterials. 1992;13:308-12.

[14] Yuan H, Kurashina K, de Bruijn JD, Li Y, De Groot K, Zhang X. A preliminary study on osteoinduction of two kinds of calcium phosphate ceramics. Biomaterials. 1999;20:1799-806.

[15] Hing KA, Revell PA, Smith N, Buckland T. Effect of silicon level on rate, quality and progression of bone healing within silicate-substituted porous hydroxyapatite scaffolds. Biomaterials. 2006;27:5014-26.

[16] Davison N, Luo X, Schoenmaker T, Everts V, Yuan H, Barrère-de Groot F, et al. Submicron-scale surface architecture of tricalcium phosphate directs osteogenesis in vitro and in vivo. European cells \& materials. 2013;27:281-97.

[17] Zhang J, Luo X, Barbieri D, Barradas A, de Bruijn JD, van Blitterswijk CA, et al. The size of surface microstructures as an osteogenic factor in calcium phosphate ceramics. Acta biomaterialia. 2014;10:3254-63.

[18] Wang H, Zhi W, Lu X, Li X, Duan K, Duan R, et al. Comparative studies on ectopic bone formation in porous hydroxyapatite scaffolds with complementary pore structures. Acta biomaterialia. 2013;9:8413-21.

[19] Barradas AM, Fernandes HA, Groen N, Chai YC, Schrooten J, van de Peppel J, et al. A calcium-induced signaling cascade leading to osteogenic differentiation of human bone marrow-derived mesenchymal stromal cells. Biomaterials. 2012;33:3205-15.

[20] Yuan H, Fernandes H, Habibovic P, De Boer J, Barradas A, De Ruiter A, et al. Osteoinductive ceramics as a synthetic alternative to autologous bone grafting. Proceedings of the National Academy of Sciences of the United States of America. 2010;107:13614-9.

[21] Fujibayashi S, Neo M, Kim H-M, Kokubo T, Nakamura T. Osteoinduction of porous bioactive titanium metal. Biomaterials. 2004;25:443-50. 
[22] Marques P, Serro A, Saramago B, Fernandes A, Magalhaes M, Correia R. Mineralisation of two phosphate ceramics in HBSS: role of albumin. Biomaterials. 2003;24:451-60.

[23] Garnett J, Dieppe P. The effects of serum and human albumin on calcium hydroxyapatite crystal growth. Biochem J. 1990;266:863-8.

[24] Urist MR, Huo YK, Brownell AG, Hohl WM, Buyske J, Lietze A, et al. Purification of bovine bone morphogenetic protein by hydroxyapatite chromatography. Proceedings of the National Academy of Sciences of the United States of America. 1984;81:371.

[25] Zhang J, Barbieri D, ten Hoopen H, de Bruijn JD, van Blitterswijk CA, Yuan H. Microporous calcium phosphate ceramics driving osteogenesis through surface architecture. Journal of Biomedical Materials Research Part A. 2014:n/a-n/a.

[26] Cuaranta-Monroy I, Simandi Z, Kolostyak Z, Doan-Xuan Q-M, Poliska S, Horvath A, et al. Highly efficient differentiation of embryonic stem cells into adipocytes by ascorbic acid. Stem Cell Research. 2014;13:88-97.

[27] Keck M, Kober J, Riedl O, Kitzinger HB, Wolf S, Stulnig TM, et al. Power assisted liposuction to obtain adipose-derived stem cells: Impact on viability and differentiation to adipocytes in comparison to manual aspiration. Journal of Plastic, Reconstructive \& Aesthetic Surgery. 2014;67:e1-e8.

[28] Meretoja VV, Dahlin RL, Wright S, Kasper FK, Mikos AG. The effect of hypoxia on the chondrogenic differentiation of co-cultured articular chondrocytes and mesenchymal stem cells in scaffolds. Biomaterials. 2013;34:4266-73.

[29] Zheng X, Wu Y, Zhu L, Chen Q, Zhou Y, Yan H, et al. Angiotensin II promotes differentiation of mouse embryonic stem cells to smooth muscle cells through PI3-kinase signaling pathway and NF-KB. Differentiation. 2013;85:41-54.

[30] Barradas AM, Monticone V, Hulsman M, Danoux C, Fernandes H, Tahmasebi Z, et al. Molecular mechanisms of biomaterial-driven osteogenic differentiation in human mesenchymal stromal cells. Integrative biology : quantitative biosciences from nano to macro. 2013;5:920-31.

[31] Song G, Habibovic P, Bao C, Hu J, van Blitterswijk CA, Yuan H, et al. The homing of bone marrow MSCs to non-osseous sites for ectopic bone formation induced by osteoinductive calcium phosphate. Biomaterials. 2013.

[32] Barradas AM, Monticone V, Hulsman M, Danoux C, Fernandes H, Tahmasebi Birgani Z, et al. Molecular mechanisms of biomaterial-driven osteogenic differentiation in human mesenchymal stromal cells. Integrative biology : quantitative biosciences from nano to macro. 2013;5:920-31.

[33] Pockwinse SM, Stein JL, Lian JB, Stein GS. Developmental stage-specific cellular responses to vitamin D and glucocorticoids during differentiation of the osteoblast phenotype: interrelationship of morphology and gene expression by in situ hybridization. Experimental Cell Research. 1995;216:244-60.

[34] Sikavitsas VI, Temenoff JS, Mikos AG. Biomaterials and bone mechanotransduction. Biomaterials. 2001;22:2581-93.

[35] Carvalho R, Schaffer J, Gerstenfeld L. Osteoblasts induce osteopontin expression in response to attachment on fibronectin: demonstration of a common role for integrin receptors in the signal transduction processes of cell attachment and mechanical stimulation. Journal of cellular biochemistry. 1998;70:376-90.

[36] Rodríguez Fernández JL, Ben-Ze'ev A. Regulation of fibronectin, integrin and cytoskeleton expression in differentiating adipocytes: inhibition by extracellular matrix and polylysine. Differentiation. 1989;42:65-74.

[37] Eggenschwiler JT, Anderson KV. Cilia and developmental signaling. Annual review of cell and developmental biology. 2007;23:345.

[38] McMurray R, Wann A, Thompson C, Connelly J, Knight M. Surface topography regulates wnt signaling through control of primary cilia structure in mesenchymal stem cells. Scientific reports. 2013;3.

[39] Horani A, Brody SL, Ferkol TW, Shoseyov D, Wasserman MG, Ta-shma A, et al. CCDC65 mutation causes primary ciliary dyskinesia with normal ultrastructure and hyperkinetic cilia. PLoS One. 2013;8:e72299.

[40] Lienkamp S, Ganner A, Walz G. Inversin, Wnt signaling and primary cilia. Differentiation. 2012;83:S49-S55.

[41] Goetz SC, Anderson KV. The primary cilium: a signalling centre during vertebrate development. Nature Reviews Genetics. 2010;11:331-44.

[42] Yuan H, Van Blitterswijk C, De Groot K, De Bruijn J. A comparison of bone formation in biphasic calcium phosphate (BCP) and hydroxyapatite (HA) implanted in muscle and bone of dogs at different time periods. Journal of Biomedical Materials Research Part A. 2006;78:139-47. 


\section{Chapter 7}

[43] Wang X, Wang Y, Gou W, Lu Q, Peng J, Lu S. Role of mesenchymal stem cells in bone regeneration and fracture repair: a review. International orthopaedics. 2013;37:2491-8.

[44] Davison NL, Gamblin A-L, Layrolle P, Yuan H, de Bruijn JD, Barrère-de Groot F. Liposomal clodronate inhibition of osteoclastogenesis and osteoinduction by submicrostructured beta-tricalcium phosphate. Biomaterials. 2014;35:5088-97.

[45] Davison NL, ten Harkel B, Schoenmaker T, Luo X, Yuan H, Everts V, et al. Osteoclast resorption of betatricalcium phosphate controlled by surface architecture. Biomaterials. 2014;35:7441-51.

[46] Doherty MJ, Canfield AE. Gene expression during vascular pericyte differentiation. Critical reviews in eukaryotic gene expression. 1998;9:1-17.

[47] Doherty MJ, Ashton BA, Walsh S, Beresford JN, Grant ME, Canfield AE. Vascular pericytes express osteogenic potential in vitro and in vivo. Journal of Bone and Mineral Research. 1998;13:828-38.

[48] Marinucci L, Balloni S, Becchetti E, Bistoni G, Calvi EM, Lumare E, et al. Effects of hydroxyapatite and Biostite ${ }^{\circledR}$ on osteogenic induction of hMSC. Annals of biomedical engineering. 2010;38:640-8. 


\section{Summary}

The incidence of patients which require spinal fusion or bone regeneration in large bone defects caused by trauma, tumors, tumor resection, infections or abnormal skeletal development, is on the rise. Traditionally, in both spinal fusion surgery and other bone regeneration approaches, bone grafts are applied. Even though autografts have shown to provide the best performance in bone regeneration till date, it is of great need to develop and improve alternatives to overcome the limited availability of autografts.

Being chemically similar to the inorganic components of hard tissues, calcium phosphate (CaP) ceramics are long been evaluated as synthetic bone grafting materials. Most recently, it has been found that the bone forming ability of CaP ceramics could be improved through physico-chemical modification. For the best, CaP ceramics could be made osteoinductive and such osteoinductive CaP ceramics could aid in the repair of critical-sized bone defects.

In this thesis, both in vitro cell culture and an in vivo osteoinduction study model (i.e. ectopic implantation) where applied to study the role of physico-chemical properties of $\mathrm{CaP}$ ceramics - with the emphasis on the role of surface structures - in bone formation.

The response of human bone marrow mesenchymal stromal cells (hBMSCs) in vitro and osteoinductive capacity in vivo was assessed on two types of tricalcium phosphate (TCP) ceramics with various surface structure dimensions using both a 3D (Chapter 2) and 2D system (Chapter 3 ). From these studies, both in vitro and in vivo, a clear effect of surface structure dimensions on osteogenesis was observed (Chapter 2 and Chapter 3). The results from Chapter 2 further pointed out that surface structure of CaP ceramics may enhance bone formation via surface topography instead of via changes in the chemical cues in the culture medium which has been studied using a transwell model. To further prove the role of surface topography in bone formation, gold and carbon coatings were applied to both surface structured CaP ceramics surfaces and smooth glass slides. The results confirmed the hypothesis that the enhanced osteogenesis of hBMSCs was indeed dominantly triggered by the surface structure (Chapter 4), the surface chemistry did not play a major role. Moreover, the importance of surface topography in osteogenesis was proven via the inductive bone 


\section{Summary}

formation capacity of CaP ceramics which were provoking the same chemical changes (e.g. protein adsorption, ion release and surface re-precipitation) but having different surface topographical cues (Chapter 6).

To study how surface topography directs osteogenic differentiation of cells, cell morphology and primary cilia development were investigated in vitro for hBMSCs (Chapter 3 ). The results showed that surface topography could have comparable osteogenic function as conventionally applied osteogenic inducible molecules (i.e. dexamethasone). This indicated that hBMSCs may adapt their morphology according to surface structure and develop their primary cilia which are coupled to the pathways of osteogenic differentiation.

Bone regeneration is a complex process and stem cells may not be the only cells responding to surface topography in enhanced bone formation in submicron surface structured $\mathrm{CaP}$ ceramics. Chapter 5 shows the response of other bone related cells on surface structures of CaP ceramics. Six types of cells (pericytes, inducible myoblasts and osteogenic cells at various maturation states) were cultured on TCP ceramics with various surface structure dimensions. The results revealed that both inducible cells and osteogenic cells at different mature stages are sensitive to surface topography, although the different cell types showed various responses. For example, the surface structure affected the osteogenic differentiation of inducible cells, osteoprogenitor cells and immature osteoblasts, but in the case of mature osteoblast the proliferation rate was affected.

This thesis distinguished the role of physical cues from physico-chemical properties of $\mathrm{CaP}$ ceramics in osteogenesis, and clarified the role of surface topography in bone regeneration. The overall results justified the strategy of improving bone regeneration capacity of synthetics using smart design of surface structures and specifically the dimension of the surface structure. 


\section{Samenvatting}

Het aantal patiënten dat een spinale fusie of een andere ingreep ter regeneratie van grote botdefecten behoeft, neemt toe. Deze botdefecten kunnen het gevolg zijn van trauma, tumor resectie, infecties of abnormale skeletale ontwikkeling. In zowel spinale fusie als in andere bot regeneratie methoden, wordt er doorgaans gebruik gemaakt van zogenaamde botvulmaterialen (ook wel: bone grafts). Tot op heden zijn de beste resultaten geboekt met behulp van bone grafts van autologe origine. Desondanks is er grote vraag naar de ontwikkeling en verbetering van alternatieven om de problematiek gerelateerd aan de beperkte beschikbaarheid van autoloog materiaal te omzeilen.

Calciumfosfaten (CaP's) zijn lange tijd onderzocht als potentieel botvulmateriaal, door hun chemische overeenkomstigheid met de inorganische componenten van hard weefsel. Recentelijk onderzoek heeft uitgewezen dat de botvormende potentiaal van CaP verbeterd kan worden door middel van fysico-chemische modificaties. In een optimale situatie zouden CaP's osteoinductief gemaakt kunnen worden en kunnen bijdragen aan het herstel van botdefecten van kritieke grootte.

In dit proefschrift staan de resultaten van zowel in vitro kweek experimenten als in vivo modellen voor osteoinductie (bijv. ectopische implantatie) beschreven. Deze experimenten zijn uitgevoerd om de rol van fysico-chemische eigenschappen, met in het bijzonder de rol van oppervlakte structuur, van de CaP's te bestuderen.

De respons van humane mesenchymale stromale beemergcellen (hBMSCs) op twee typen tricalcium fosfaat (TCP) is bestudeerd door middel van een in vitro kweek en de osteoinductieve capaciteit van deze TCP keramieken is bekeken aan de hand van een in vivo studie. De twee TCP keramieken verschilden slechts in de grootte en daarmee topografie van de oppervlaktestructuur, en zijn zowel in 3D (Hoofdstuk 2) als in 2D (Hoofdstuk 3) bekeken. Zowel in vitro als in vivo is een duidelijk effect van de oppervlakte structuur van de keramieken op het proces van osteogenese waarneembaar. Uit de resultaten beschreven in Hoofdstuk 2 kan geconcludeerd worden dat de oppervlakte structuur van CaP keramieken 


\section{Samenvatting}

bot formatie kan bevorderen, terwijl een mogelijk effect van veranderingen in chemische factoren in het kweekmedium is uitgesloten aan de hand van een zogenaamd transwellexperiment. Om de rol van oppervlakte structuur in botformatie te bewijzen zijn goud en koolstofcoatings aangebracht op het oppervlak van de CaP keramieken en ter controle op glasplaatjes. De resultaten van in vitro kweek van hBMSCs op deze gecoate materialen bevestigen de hypothese dat de verbeterde osteogene capaciteit van hBMSCs gerelateerd is aan de oppervlakte structuur (Hoofdstuk 4). Daarnaast is het belang van de veranderde oppervlakte structuur ook bewezen in het proces van osteogenese door analyse van de osteoinductieve bot formatie capaciteit van de keramieken. Van deze CaP keramieken is aangetoond dat ze dezelfde chemische veranderingen ondergaan tijdens het experiment (bijv. eiwit adsorptie, ion afgifte en oppervlakte re-precipitatie), maar dat ze een verschillende topografische oppervlakte structuur bevatten (Hoofdstuk 6).

Cel morfologie van de hBMSCs en de aanwezigheid van primaire cilia op hBMSCs werden als maat voor osteogene functie in vitro bestudeerd (Hoofdstuk 3). De resultaten laten zien dat de oppervlakte topografie een osteogene functie oplevert die vergelijkbaar is met de functie verkregen na toediening van de conventionele toegepaste moleculen in het kweekmedium (bijv. dexamethason). Dit wekt de indruk dat hBMSCs hun morfologie aanpassen aan de oppervlakte structuur en dat ze hun primaire cilia ontwikkelen zoals dit bekend is in processen met betrekking tot osteogene differentiatie.

Bot regeneratie is een complex proces waarbij naast stromale cellen wellicht ook andere celtypen reageren op de oppervlakte structuur van bone grafts. In Hoofdstuk 5 is de respons van diverse andere celtypen gerelateerd aan botweefsel bestudeerd. Zes celtypen (bijv. pericyten, induceerbare myoblasten en osteogene cellen in verschillende fasen van maturiteit) zijn op TCP keramieken met verschillende groottes van oppervlakte structuur gekweekt. De resultaten tonen aan dat zowel induceerbare cellen als osteogene cellen van verschillende fasen van maturiteit gevoelig zijn voor de oppervlakte topografie, al zij het met verschillende typen respons. Zo werd bijvoorbeeld de osteogene differentiatie beïnvloed in induceerbare cellen, osteogene voorloper-cellen en ongematureerde osteoblasten, terwijl 
voor gematureerde osteoblasten vooral een verschil in de proliferatie snelheid werd gevonden.

In dit proefschrift zijn de rol van fysische en fysico-chemische eigenschappen van CaP keramieken in osteogenese onderscheiden en is de rol van de oppervlakte structuur van CaP in bot regeneratie aangetoond. De resultaten beschreven in dit proefschrift verantwoorden de strategie om bot regeneratie capaciteit van materialen te verbeteren door oppervlakte structuur, en in het bijzonder de grootte van de oppervlakte structuur, te ontwerpen en te optimaliseren. 



\section{List of Publications and Selected Abstracts}

\section{Publications related to this thesis}

Zhang J, Barbieri D, ten Hoopen H, de Bruijn JD, van Blitterswijk CA, Yuan H. Microporous calcium phosphate ceramics driving osteogenesis through surface architecture. Journal of Biomedical Materials Research Part A. 2015;3:1188-99

> Zhang J, Luo X, Barbieri D, Barradas A, de Bruijn JD, van Blitterswijk CA, Yuan H. The size of surface microstructures as an osteogenic factor in calcium phosphate ceramics. Acta Biomaterialia. 2014;10:3254-63.

> Zhang J, De Sousa FV, Carriche GAM, Luo X, Barbieri D, Damanik F, van den Beucken J, Moroni L, de Bruijn JD, van Blitterswijk CA, Yuan H. Calcium phosphate ceramics initiate osteogenic response through topographical cues (Submitted to European cells and materials).

> Zhang J, Sun L, Luo X, Barbieri D, Damanik F, Moroni L, de Bruijn JD, van Blitterswijk $\mathrm{CA}$, Yuan $\mathrm{H}$. Cells responding to surface structure of calcium phosphate ceramics for bone regeneration (Submitted to Journal of Tissue Engineering and Regenerative Medicine)

> Zhang J, Dalbay M, Luo X, Vrij E, Barbieri D, Kngiht MM, Moroni L, de Bruijn JD, van Blitterswijk CA, Yuan H. Surface structure of calcium phosphate ceramics instructs inductive bone formation via influencing on morphology and primary cilia structure of stem cells (To be submitted to Acta Biomaterialia).

\section{Selected Abstracts}

> Jingwei Zhang, Xiaoman Luo, Joost D. de Bruijn, Clemens A. van Blitterswijk, Huipin Yuan, "Surface topography of calcium phosphate ceramics affecting cellular behaviors", $20^{\text {th }}$ annual NBTE meeting, Lunteren, The Netherlands, 2011, Poster.

> Jingwei Zhang, Davide Barbieri, Jie Weng, Joost D. de Bruijn, Clemens A. van Blitterswijk, Huipin Yuan, "Microstructure initiating bone formation in calcium 
phosphate ceramic implants directly through surface roughness", $9^{\text {th }}$ world biomaterials congress, Chengdu, China, 2012, Oral presentation.

Jingwei Zhang, Davide Barbieri, Xiaoman Luo, Joost D. de Bruijn, Clemens A. van Blitterswijk, Huipin Yuan, "Dimension of surface microstrcutures as an essential element for osteoinduction of calcium phosphate ceramics", $9^{\text {th }}$ world biomaterials congress, Chengdu, China, 2012, Oral presentation, Young scientist award.

Jingwei Zhang, Davide Barbieri, Ana Barradasa, Joost D. de Bruijn, Clemens A. van Blitterswijka, Huipin Yuan, "Surface microstrcuture regulating osteogenic response through surface topography", ESB, Madrid, Spain, 2013, Oral presentation. 
I highly appericiate your kindness and supporting.

- Jingwei
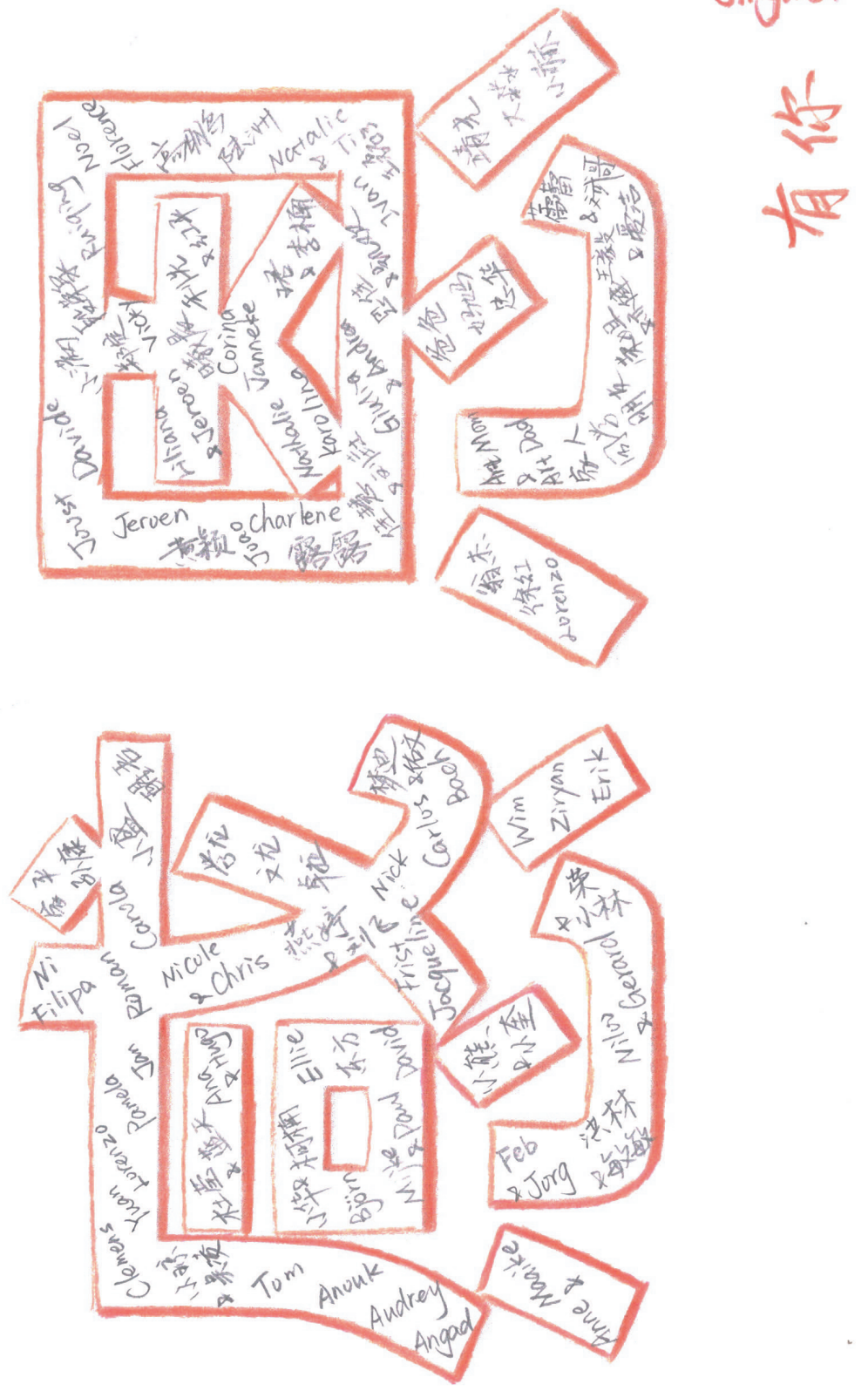



\section{Curriculum Vitea}

Jingwei Zhang was born on the April $1^{\text {st }}, 1983$ in Zhaobitun, a small village in Wuchang, Heilongjiang, China. She grew up in Zhaobitun until she was 9 year old, and she started her long journey of study. She graduated in 2001 from Wuchang High Experimental School (Wuchang, China). She then went to study on Materials and Chemistry in Tianjin Institute of Urban Construction (Tianjin, China) and get her bachelor degree in 2005 . After 3 years, in July 2008, she

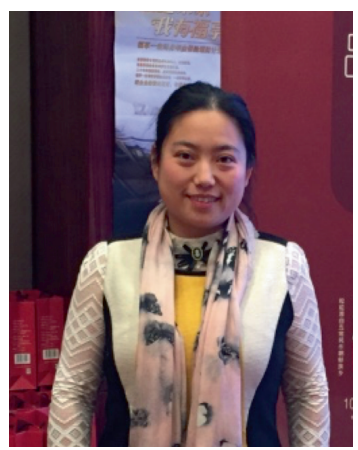
started her study in the Master-Doctor combine program under the supervision of Prof. Dr. Jie Weng in Southwest Jiaotong University (Chengdu, China) where I had wonderful time both on my study and life. In September 2010, she started as a PhD students in the department of Tissue Regeneration at University of Twente, Enschede, The Netherlands. She focused on the function of surface structure of calcium phosphate ceramics in bone regeneration under the supervision of Prof. Dr. C. A. van Blitterswijk and Dr. H. Yuan. She grew up a lot in University of Twente and finished her PhD thesis in 2016. More importantly, she harvests the friendship with both Chinese and international friends and the great memories, which are her best fortune. Now, she lives in Shanghai, China, being a happy easy girl who is selling rice with her own brand of Zhaobitun. 

ISBN:978-90-365-4062-9 